\title{
Access to Saturated Thiocyano-Containing Azaheterocycles via Selenide-Catalyzed Regio- and Stereoselective Thiocyanoaminocyclization of Alkenes
}

\author{
Wei Wei, Lihao Liao, Tian Qin, and Xiaodan Zhao* \\ Institute of Organic Chemistry \& MOE Key Laboratory of Bioinorganic and Synthetic Chemistry, School of Chemistry, \\ Sun Yat-Sen University, Guangzhou 510275, P. R. China \\ E-mail: zhaoxd3@mail.sysu.edu.cn
}

\section{Table of Contents}

1. General Considerations

2. Synthesis of the Starting Materials

3. General Procedure for the Synthesis of Saturated Thiocyano-Containing Heterocycles by Selenide Catalysis

4. Further Transformations of $\mathbf{2 a}$

5. General Procedure for Catalytic Asymmetric Synthesis of Saturated S20 Thiocyano-Containing Heterocycles

6. NMR Spectra for New Compounds 


\section{General Considerations}

Unless otherwise noted, commercial reagents were purchased from Alfa Aesar, TCI, J\&K or Adamas and used without further purification. Reaction solvents THF, $\mathrm{Et}_{2} \mathrm{O}$ and dichloromethane (DCM) and MeCN were purified by PureSolv MD-5 (Innovative Technology). All reactions were carried out using oven-dried glassware and all catalytic reactions proceeded without special care. Analytical thin layer chromatography was performed on $0.20 \mathrm{~mm}$ silica gel HSGF-254 plates (Huanghai, China), and visualized under $254 \mathrm{~nm}$ UV light or by staining with potassium permanganate. Column chromatography was performed on 200-300 mesh silica gel (Huanghai, China).

${ }^{1} \mathrm{H},{ }^{19} \mathrm{~F}$ and ${ }^{13} \mathrm{C}\left\{{ }^{1} \mathrm{H}\right\}$ NMR spectra were recorded on an AVANCE III $400 \mathrm{MHz}$ spectrometer at ambient temperature. ${ }^{1} \mathrm{H}$ NMR spectra are referred to the TMS signal $(\delta=0 \mathrm{ppm})$ and ${ }^{13} \mathrm{C}$ NMR spectra are referred to the residual solvent signal $(\delta=77.16 \mathrm{ppm})$. Data for ${ }^{1} \mathrm{H}$ NMR are reported as follows: chemical shifts $(\delta \mathrm{ppm})$, multiplicities $(\mathrm{s}=$ singlet, $\mathrm{d}=$ doublet, $\mathrm{t}=$ triplet, $\mathrm{q}=$ quartet, $\mathrm{m}=$ multiplet, br $=$ broad $)$, coupling constants $(\mathrm{Hz})$, integration. Data for ${ }^{13} \mathrm{C}\left\{{ }^{1} \mathrm{H}\right\}$ NMR and ${ }^{19} \mathrm{~F}$ NMR are reported as follows: chemical shift $(\delta \mathrm{ppm})$, multiplicity $(\mathrm{q}=$ quartet $)$, coupling constant $(\mathrm{Hz})$.

High resolution mass spectra of new compounds were recorded on LTQ Orbitrap Elite LC/MS (ESI) or MAT 95XP (Thermo, EI) at analytical center of Sun Yat-Sen University, and Guangzhou Micromon Technology Services CO. LTD. Enantiomeric excesses were determined by HPLC analysis on Shimadzu HPLC units including the following instruments: LC-20AT pump, SPD-M20A detector and Daciel Chiralpak ID column.

Thiocyanating reagents, $N$-trifluoromethylthiosaccharin, and all chalcogenide catalysts were known and were prepared according to the known procedures. ${ }^{1,2}$

\section{Synthesis of the Starting Materials}

Substrates $\mathbf{1 c}, \mathbf{1} \mathbf{i}, \mathbf{1 j}, \mathbf{1 n}, \mathbf{1 p}-\mathbf{1 r}, \mathbf{1 t}-\mathbf{1 x}$ were prepared according to the known procedures, and their NMR data are in agreement with those described in the literature. ${ }^{2 \mathrm{a}, 3}$
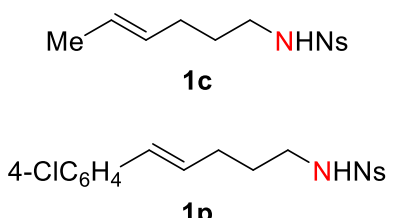

$1 \mathrm{p}$<smiles>OCC/C=C/c1ccccc1</smiles>

1u

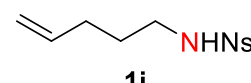

$1 \mathbf{i}$

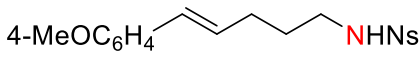

$1 q$

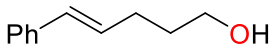

$1 \mathrm{v}$

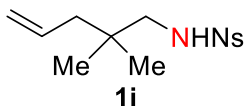

$\mathrm{Me}$
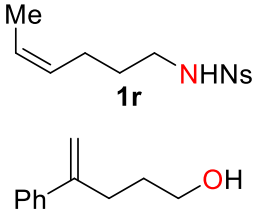

$1 \mathrm{w}$
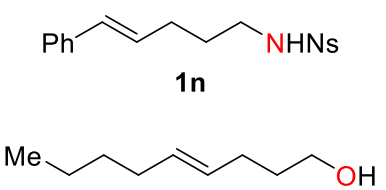

$1 \mathrm{t}$<smiles>C=C(CCC(=O)O)c1ccccc1</smiles>

$1 \mathrm{x}$ 


\subsection{General procedure for the preparation of substrates}

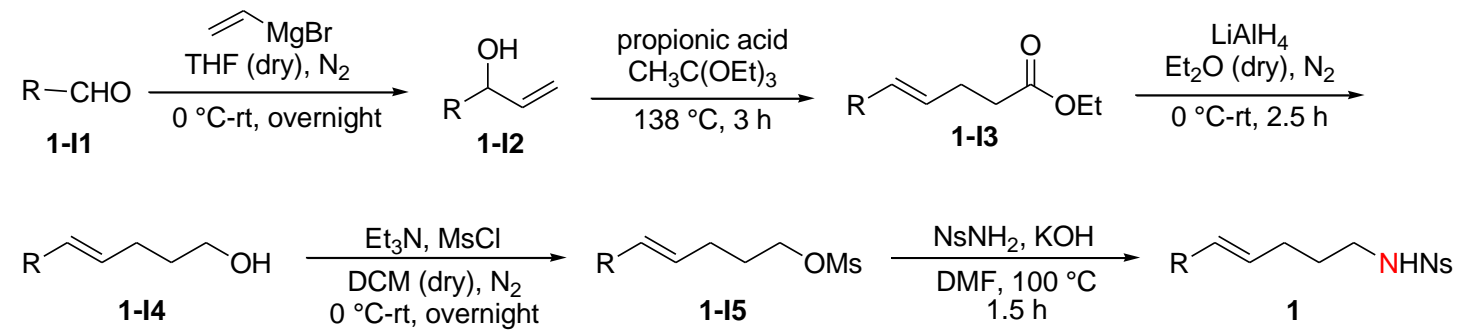

Sulfonamides 1a, 1b, 1d-1h, and 1o were synthesized according to the procedure in the literature. $^{4}$

To a stirred solution of vinyl magnesium bromide-THF $(6 \mathrm{~mL}, 1 \mathrm{M}$ solution, $5.0 \mathrm{~mol})$ at $0{ }^{\circ} \mathrm{C}$ was added aldehyde 1-I1 $(5.0 \mathrm{mmol})$ in dry THF $(4 \mathrm{~mL})$ dropwise under a nitrogen atmosphere within $10 \mathrm{~min}$. The solution was allowed to warm up to at room temperature and stirred for $12 \mathrm{~h}$. Saturated $\mathrm{NH}_{4} \mathrm{CI}$ solution $(15 \mathrm{ml})$ was then added. The resulting mixture was extracted with DCM $(15 \mathrm{~mL} \times 3)$. The combined organic layers were washed with brine $(15 \mathrm{~mL})$, dried over anhydrous $\mathrm{Na}_{2} \mathrm{SO}_{4}$, and concentrated in vacuo. The residue was purified by silica gel column chromatography (eluent: PE/EA = 10:1) to afford allyl alcohol 1-I2.

A solution of 1-I2 (4.5 mmol), propionic acid $(20.1 \mu \mathrm{L}, 6 \mathrm{~mol} \%)$, and triethyl orthoacetate (5.8 $\mathrm{mL}, 31.5 \mathrm{mmol}$ ) was stirred at $138{ }^{\circ} \mathrm{C}$ for $3 \mathrm{~h}$. The resulting solution was then concentrated in vacuo. The residue was subjected to flash silica gel column chromatography (eluent: $\mathrm{PE} / \mathrm{EA}=40: 1$ ) to afford the desired ethyl ester product 1-I3.

To a suspension of $\mathrm{LiAlH}_{4}(303.6 \mathrm{mg}, 8.0 \mathrm{mmol})$ in dry $\mathrm{Et}_{2} \mathrm{O}(12 \mathrm{~mL})$ was added ester $\mathbf{1}-\mathbf{I} 3$ (4.0 $\mathrm{mmol}$ in $4 \mathrm{~mL}$ dry $\mathrm{Et}_{2} \mathrm{O}$ ) dropwise at $0{ }^{\circ} \mathrm{C}$ under a nitrogen atmosphere. The resulting mixture was stirred at $0{ }^{\circ} \mathrm{C}$ for $10 \mathrm{~min}$, after which it was warmed up to room temperature and stirred for additional $2.5 \mathrm{~h}$. After the complete consumption of the starting material (monitored by TLC), the reaction was quenched with ice carefully at $0{ }^{\circ} \mathrm{C}$. The mixture was filtered through anhydrous $\mathrm{Na}_{2} \mathrm{SO}_{4}$ and the residue in funnel was washed with DCM $(60 \mathrm{~mL})$. Evaporation of the solvents left a crude mixture, which was purified by flash silica gel column chromatography (eluent: PE/EA = 3:1) to give alcohol 1-I4.

To a solution of alcohol 1-I4 $(3.5 \mathrm{mmol})$ and triethylamine $(737 \mu \mathrm{L}, 5.3 \mathrm{mmol})$ in dry DCM (15 $\mathrm{mL})$ at $0{ }^{\circ} \mathrm{C}$ was added $\mathrm{MsCl}(302 \mu \mathrm{L}, 3.9 \mathrm{mmol})$ under a nitrogen atmosphere. The resulting mixture was stirred at $0{ }^{\circ} \mathrm{C}$ for $10 \mathrm{~min}$, after which it was warmed up to room temperature and stirred overnight. Then the reaction was quenched with water $(15 \mathrm{~mL})$ and extracted with DCM (15 $\mathrm{mL} \times 3)$. The combined organic extracts were dried over anhydrous $\mathrm{Na}_{2} \mathrm{SO}_{4}$, filtered, and concentrated. The residue was purified by flash column chromatography to (eluent: $\mathrm{PE} / \mathrm{EA}=3: 1$ ) give compound 1-I5. 
A solution of $\mathrm{NsNH}_{2}(303.0 \mathrm{mg}, 1.5 \mathrm{mmol})$ and $\mathrm{KOH}(84.0 \mathrm{mg}, 1.5 \mathrm{mmol})$ in DMF (5 mL) was stirred at $100{ }^{\circ} \mathrm{C}$ for $0.5 \mathrm{~h}$. Then, to the resulting mixture was added a solution of compound 1-I5 $(1.0 \mathrm{mmol})$ in DMF $(1 \mathrm{~mL})$ dropwise. After addition, the mixture was stirred at $100{ }^{\circ} \mathrm{C}$ for additional $1 \mathrm{~h}$, and then cooled to room temperature. The reaction mixture was diluted with water $(15 \mathrm{~mL})$ and extracted with DCM $(15 \mathrm{~mL} \times 3)$. The combined organic extracts were washed with water $(15 \mathrm{~mL} \times$ 3 ) and brine $(15 \mathrm{~mL})$, dried over anhydrous $\mathrm{Na}_{2} \mathrm{SO}_{4}$, filtered, and concentrated in vacuo. The residue was purified by flash column chromatography to give sulfonamide $\mathbf{1}$.

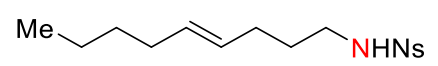

(E)-4-Nitro- $N$-(non-4-en-1-yl)benzenesulfonamide (1a): Flash column chromatography (eluent: $\mathrm{PE} / \mathrm{EtOAc}=20 / 1$ to $5 / 1, \mathrm{v} / \mathrm{v}$ ) to afford $1 \mathrm{a}$ as a yellow solid, $1632.6 \mathrm{mg}, 60 \%$ yield in the last step with 8.4 mmol 1-I5. ${ }^{1} \mathrm{H}$ NMR (400 MHz, $\left.\mathrm{CDCl}_{3}\right) \delta 8.44-8.30(\mathrm{~m}, 2 \mathrm{H}), 8.12-8.00$ (m, 2H), $5.42-$ $5.33(\mathrm{~m}, 1 \mathrm{H}), 5.31-5.22(\mathrm{~m}, 1 \mathrm{H}), 4.83-4.69(\operatorname{broad} \mathrm{s}, 1 \mathrm{H}), 3.02(\mathrm{q}, J=6.7 \mathrm{~Hz}, 2 \mathrm{H}), 2.02-1.90(\mathrm{~m}$, $4 \mathrm{H}), 1.55(\mathrm{~m}, 2 \mathrm{H}), 1.31-1.24(\mathrm{~m}, 4 \mathrm{H}), 0.87(\mathrm{t}, J=6.9 \mathrm{~Hz}, 3 \mathrm{H}) .{ }^{13} \mathrm{C} \mathrm{NMR}\left(101 \mathrm{MHz}, \mathrm{CDCl}_{3}\right) \delta$ $150.18,146.18,132.41,128.41,128.13,124.52,42.98,32.29,31.74,29.55,29.48,22.30,14.04$. HR-ESI-MS $m / z$ calcd. for $\mathrm{C}_{15} \mathrm{H}_{21} \mathrm{~N}_{2} \mathrm{O}_{4} \mathrm{~S}$ [M - H]: 325.1228 , found: 325.1235 .

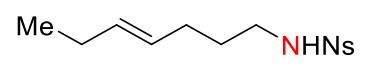

$N$-(Hept-4-en-1-yl)-4-nitrobenzenesulfonamide (1b). Flash column chromatography (eluent: $\mathrm{PE} / \mathrm{EtOAc}=5 / 1, \mathrm{v} / \mathrm{v}$ ) to afford $\mathbf{1 b}$ as a yellow solid, $287.8 \mathrm{mg}, 67 \%$ yield in the last step with 1.4 mmol 1-I5. ${ }^{1} \mathrm{H}$ NMR (400 MHz, $\left.\mathrm{CDCl}_{3}\right) \delta 8.46$ - $8.31(\mathrm{~m}, 2 \mathrm{H}), 8.13-8.01(\mathrm{~m}, 2 \mathrm{H}), 5.48-5.21(\mathrm{~m}$, 2H), $5.03-4.66(\mathrm{~m}, 1 \mathrm{H}), 3.02(\mathrm{dd}, J=13.2,7.0 \mathrm{~Hz}, 2 \mathrm{H}), 2.03-1.92(\mathrm{~m}, 4 \mathrm{H}), 1.55(\mathrm{~m}, 2 \mathrm{H}), 0.93(\mathrm{t}$, $J=7.5 \mathrm{~Hz}, 3 \mathrm{H}) .{ }^{13} \mathrm{C} \mathrm{NMR}\left(101 \mathrm{MHz}, \mathrm{CDCl}_{3}\right) \delta 150.17,146.15,133.88,128.41,127.20,124.53$, 42.98, 29.51, 29.45, 25.63, 13.93. HR-ESI-MS m/z calcd. for $\mathrm{C}_{13} \mathrm{H}_{17} \mathrm{~N}_{2} \mathrm{O}_{4} \mathrm{~S}[\mathrm{M}-\mathrm{H}]^{-}:$297.0915, found: 297.0921.

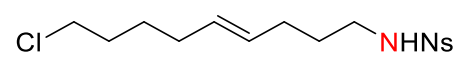

$N$-(9-Chloronon-4-en-1-yl)-4-nitrobenzenesulfonamide (1d). Flash column chromatography (eluent: PE/EtOAc $=20: 1$ to 6/1, v/v) to afford 1d as a yellow solid, $579.4 \mathrm{mg}, 59 \%$ yield in the last step with 2.7 mmol 1-I5. ${ }^{1} \mathrm{H}$ NMR $\left(400 \mathrm{MHz}, \mathrm{CDCl}_{3}\right) \delta 8.41-8.34(\mathrm{~m}, 2 \mathrm{H}), 8.09-8.04(\mathrm{~m}, 2 \mathrm{H})$, $5.41-5.27(\mathrm{~m}, 2 \mathrm{H}), 4.81-4.68(\mathrm{~m}, 1 \mathrm{H}), 3.53(\mathrm{t}, J=6.7 \mathrm{~Hz}, 2 \mathrm{H}), 3.02(\mathrm{dd}, J=13.3,6.9 \mathrm{~Hz}, 2 \mathrm{H})$, $2.03-1.95(\mathrm{~m}, 4 \mathrm{H}), 1.78-1.71(\mathrm{~m}, 2 \mathrm{H}), 1.60-1.53(\mathrm{~m}, 2 \mathrm{H}), 1.51-1.44(\mathrm{~m}, 2 \mathrm{H}) .{ }^{13} \mathrm{C}$ NMR $(101$ $\left.\mathrm{MHz}, \mathrm{CDCl}_{3}\right) \delta 150.18,146.13,131.42,128.97,128.41,124.54,45.09,42.95,32.13,31.79,29.48$, 26.71. HR-ESI-MS m/z calcd. for $\mathrm{C}_{15} \mathrm{H}_{21} \mathrm{ClN}_{2} \mathrm{NaO}_{4} \mathrm{~S}$ [M + Na] $]^{+}: 383.0803$, found: 383.0802 .<smiles>CC(C)C/C=C/CCCNN</smiles>

$N$-(7-Methyloct-4-en-1-yl)-4-nitrobenzenesulfonamide (1e). Flash column chromatography (eluent: 
$\mathrm{PE} / \mathrm{EtOAc}=20: 1$ to $8 / 1, \mathrm{v} / \mathrm{v}$ ) to afford $1 \mathrm{e}$ as a yellow solid, $910.3 \mathrm{mg}$, 59\% yield in the last step with 4.7 mmol 1-I5. ${ }^{1} \mathrm{H}$ NMR (400 MHz, $\left.\mathrm{CDCl}_{3}\right) \delta 8.37(\mathrm{~d}, J=8.5 \mathrm{~Hz}, 2 \mathrm{H}), 8.09-8.02(\mathrm{~m}, 2 \mathrm{H}), 5.41-$ $5.20(\mathrm{~m}, 2 \mathrm{H}), 4.94-4.73(\mathrm{~m}, 1 \mathrm{H}), 3.05-2.98(\mathrm{~m}, 2 \mathrm{H}), 2.00(\mathrm{q}, J=6.8 \mathrm{~Hz}, 2 \mathrm{H}), 1.83(\mathrm{t}, J=6.5 \mathrm{~Hz}$, $2 \mathrm{H}), 1.60-1.50(\mathrm{~m}, 3 \mathrm{H}), 0.85-0.80(\mathrm{~m}, 6 \mathrm{H}) .{ }^{13} \mathrm{C} \mathrm{NMR}\left(101 \mathrm{MHz}, \mathrm{CDCl}_{3}\right) \delta 150.16,146.13$, 131.03, 129.31, 128.41, 124.52, 42.95, 41.98, 29.55, 29.49, 28.45, 22.34. HR-ESI-MS $\mathrm{m} / z$ calcd. for $\mathrm{C}_{15} \mathrm{H}_{21} \mathrm{~N}_{2} \mathrm{O}_{4} \mathrm{~S}[\mathrm{M}-\mathrm{H}]^{-}: 325.1228$, found: 325.1231 .

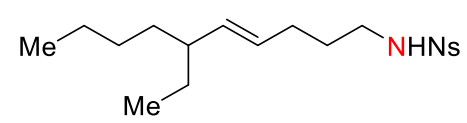

$N$-(6-Ethyldec-4-en-1-yl)-4-nitrobenzenesulfonamide (1f). Flash column chromatography (eluent: $\mathrm{PE} / \mathrm{EtOAc}=20: 1$ to $8 / 1, \mathrm{v} / \mathrm{v}$ ) to afford $\mathbf{1 f}$ as a yellow solid, $969.3 \mathrm{mg}, 64 \%$ yield in the last step with $4.1 \mathrm{mmol} \mathrm{1-I5.}{ }^{1} \mathrm{H} \mathrm{NMR}\left(400 \mathrm{MHz}, \mathrm{CDCl}_{3}\right) \delta 8.45-8.31(\mathrm{~m}, 2 \mathrm{H}), 8.11-8.01(\mathrm{~m}, 2 \mathrm{H}), 5.26-5.05$ $(\mathrm{m}, 2 \mathrm{H}), 4.90-4.71(\mathrm{br}, \mathrm{s}, 1 \mathrm{H}), 3.03(\mathrm{q}, J=6.9 \mathrm{~Hz}, 2 \mathrm{H}), 2.01(\mathrm{q}, J=7.0 \mathrm{~Hz}, 2 \mathrm{H}), 1.79-1.70(\mathrm{~m}$, $1 \mathrm{H}), 1.57(\mathrm{~m}, J=7.1 \mathrm{~Hz}, 2 \mathrm{H}), 1.38-1.12(\mathrm{~m}, 8 \mathrm{H}), 0.85(\mathrm{t}, J=7.1 \mathrm{~Hz}, 3 \mathrm{H}), 0.77(\mathrm{t}, J=7.4 \mathrm{~Hz}, 3 \mathrm{H})$. ${ }^{13} \mathrm{C} \mathrm{NMR}\left(101 \mathrm{MHz}, \mathrm{CDCl}_{3}\right) \delta 150.15,146.18,136.81,128.41,128.01,124.52,44.60,42.96,34.79$, 29.64, 29.57, 28.11, 22.89, 14.23, 11.88. HR-ESI-MS $\mathrm{m} / z$ calcd. for $\mathrm{C}_{18} \mathrm{H}_{28} \mathrm{~N}_{2} \mathrm{NaO}_{4} \mathrm{~S}[\mathrm{M}+\mathrm{Na}]^{+}$: 391.1662, found: 391.1671 .

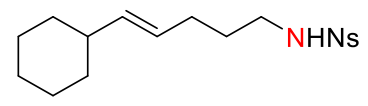

$N$-(5-Cyclohexylpent-4-en-1-yl)-4-nitrobenzenesulfonamide (1g). Flash column chromatography (eluent: $\mathrm{PE} / \mathrm{EtOAc}=15: 1$ to $6 / 1$, v/v) to afford $1 \mathrm{~g}$ as a yellow solid, $891.6 \mathrm{mg}, 62 \%$ yield in the last step with $4.1 \mathrm{mmol}$ 1-I5. ${ }^{1} \mathrm{H}$ NMR (400 $\left.\mathrm{MHz}, \mathrm{CDCl}_{3}\right) \delta 8.42-8.33$ (m, 2H), $8.11-8.02$ (m, 2H), $5.38-5.19(\mathrm{~m}, 2 \mathrm{H}), 4.87(\mathrm{t}, J=5.3 \mathrm{~Hz}, 1 \mathrm{H}), 3.01(\mathrm{dd}, J=13.2,6.9 \mathrm{~Hz}, 2 \mathrm{H}), 1.98(\mathrm{q}, J=7.0 \mathrm{~Hz}$, $2 \mathrm{H}), 1.89-1.81(\mathrm{~m}, 1 \mathrm{H}), 1.71-1.60(\mathrm{~m}, 5 \mathrm{H}), 1.55(\mathrm{p}, J=7.1 \mathrm{~Hz}, 2 \mathrm{H}), 1.27-1.10(\mathrm{~m}, 3 \mathrm{H}), 1.03-$ $0.93(\mathrm{~m}, 2 \mathrm{H}) .{ }^{13} \mathrm{C} \mathrm{NMR}\left(101 \mathrm{MHz}, \mathrm{CDCl}_{3}\right) \delta 150.15,146.13,138.39,128.42,125.61,124.52,42.93$, 40.72, 33.22, 29.60, 29.39, 26.25, 26.13. HR-ESI-MS $\mathrm{m} / \mathrm{z}$ calcd. for $\mathrm{C}_{17} \mathrm{H}_{25} \mathrm{~N}_{2} \mathrm{O}_{4} \mathrm{~S}[\mathrm{M}+\mathrm{H}]^{+}$: 353.1530, found: 353.1522 .

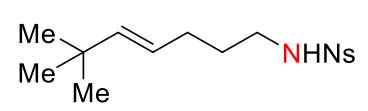

$N$-(6,6-Dimethylhept-4-en-1-yl)-4-nitrobenzenesulfonamide (1h). Flash column chromatography (eluent: $\mathrm{PE} / \mathrm{EtOAc}=20: 1$ to $6 / 1, \mathrm{v} / \mathrm{v}$ ) to afford $\mathbf{1 h}$ as a yellow solid, $441.9 \mathrm{mg}, 61 \%$ yield in the last step with $2.2 \mathrm{mmol} \mathrm{1-I5.}{ }^{1} \mathrm{H}$ NMR $\left(400 \mathrm{MHz}, \mathrm{CDCl}_{3}\right) \delta 8.42-8.32(\mathrm{~m}, 2 \mathrm{H}), 8.09-8.01$ (m, 2H), $5.48-5.36(\mathrm{~m}, 1 \mathrm{H}), 5.24-5.13(\mathrm{~m}, 1 \mathrm{H}), 4.86-4.59(\mathrm{~m}, 1 \mathrm{H}), 3.05-2.98(\mathrm{~m}, 2 \mathrm{H}), 2.02-1.95(\mathrm{~m}$, $2 \mathrm{H}), 1.59-1.53(\mathrm{~m}, 2 \mathrm{H}), 0.98-0.93(\mathrm{~m}, 9 \mathrm{H}) .{ }^{13} \mathrm{C} \mathrm{NMR}\left(101 \mathrm{MHz}, \mathrm{CDCl}_{3}\right) \delta 150.18,146.19$, 143.45, 128.41, 124.53, 122.82, 43.01, 32.97, 29.77, 29.67, 29.54. HR-ESI-MS m/z calcd. for $\mathrm{C}_{15} \mathrm{H}_{21} \mathrm{~N}_{2} \mathrm{O}_{4} \mathrm{~S}[\mathrm{M}-\mathrm{H}]^{-}: 325.1228$, found: 325.1235 . 
$\overbrace{\mathrm{NHNS}}$

4-Nitro- $N$-(5-(p-tolyl)pent-4-en-1-yl)benzenesulfonamide (10). Flash column chromatography (eluent: PE/EtOAc $=20: 1$ to 5/1, v/v) to afford 10 as a yellow solid, $353.1 \mathrm{mg}, 63 \%$ yield in the last step with $1.6 \mathrm{mmol} \mathrm{1-I5.}{ }^{1} \mathrm{H} \mathrm{NMR}\left(400 \mathrm{MHz}, \mathrm{CDCl}_{3}\right) \delta 8.31(\mathrm{~d}, J=8.8 \mathrm{~Hz}, 2 \mathrm{H}), 8.06-7.99(\mathrm{~m}, 2 \mathrm{H})$, $7.17(\mathrm{~d}, J=8.1 \mathrm{~Hz}, 2 \mathrm{H}), 7.09(\mathrm{~d}, J=8.0 \mathrm{~Hz}, 2 \mathrm{H}), 6.29(\mathrm{~d}, J=15.8 \mathrm{~Hz}, 1 \mathrm{H}), 6.05-5.95(\mathrm{~m}, 1 \mathrm{H})$, $4.81-4.66(\mathrm{~m}, 1 \mathrm{H}), 3.06(\mathrm{t}, J=7.0 \mathrm{~Hz}, 2 \mathrm{H}), 2.32(\mathrm{~s}, 3 \mathrm{H}), 2.21(\mathrm{q}, J=7.0 \mathrm{~Hz}, 2 \mathrm{H}), 1.72-1.64(\mathrm{~m}$, 2H). ${ }^{13} \mathrm{C}$ NMR $\left(101 \mathrm{MHz}, \mathrm{CDCl}_{3}\right) \delta 150.16,145.97,137.28,134.40,131.34,129.41,128.40,127.38$, 125.97, 124.53, 42.83, 29.85, 29.28, 21.27. HR-ESI-MS $m / z$ calcd. for $\mathrm{C}_{18} \mathrm{H}_{20} \mathrm{~N}_{2} \mathrm{NaO}_{4} \mathrm{~S}[\mathrm{M}+\mathrm{Na}]^{+}$: 383.1036, found: 383.1034 .

\subsection{The procedure for the preparation of sulfonamide $1 \mathrm{k}$}

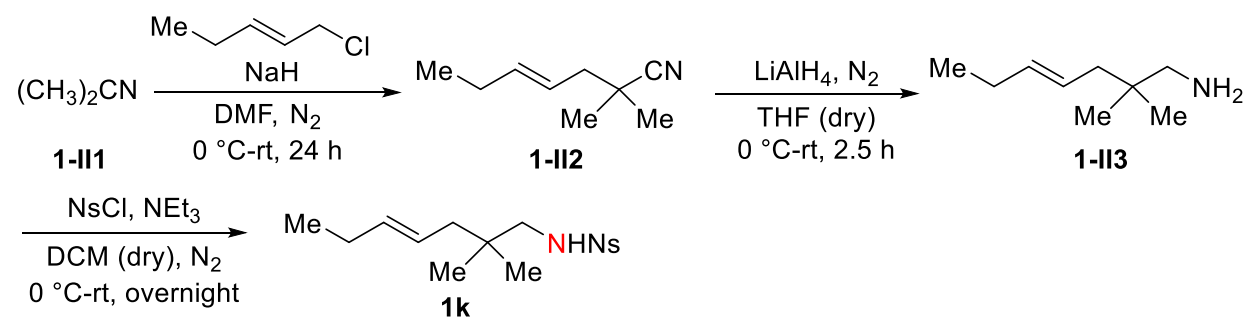

Sulfonamide 1k was synthesized according to the procedure in the literature, ${ }^{5 a}$ and (E)-1-chloropent-2-ene was prepared according to the known procedure. ${ }^{5 \mathrm{~b}}$

Under a nitrogen atmosphere, a solution of iso-butyronitrile 1-II1 (207.3 mg, $3.0 \mathrm{mmol})$ in DMF (5.0 mL) was added slowly to a suspension of $\mathrm{NaH}(180.0 \mathrm{mg}, 7.5 \mathrm{mmol})$ in DMF $(2.0 \mathrm{~mL})$ at $0{ }^{\circ} \mathrm{C}$. The resulting mixture was stirred at room temperature for $0.5 \mathrm{~h}$, after which it was cooled to 0 ${ }^{\circ} \mathrm{C}$, treated with $(E)$-1-chloropent-2-ene $(784.4 \mathrm{mg}, 7.5 \mathrm{mmol})$, warmed to room temperature, and stirred for $24 \mathrm{~h}$. Then, the mixture was poured into ice water $(15 \mathrm{~mL})$ and was extracted with $\mathrm{Et}_{2} \mathrm{O}$ $(15 \mathrm{~mL} \times 3)$. The combined organic layers were washed with water $(10 \mathrm{~mL} \times 3)$, dried with $\mathrm{Na}_{2} \mathrm{SO}_{4}$, and concentrated in vacuo to give the crude residue 1-II2, which was used directly without further purification.

To a suspension of $\mathrm{LiAlH}_{4}(170.8 \mathrm{mg}, 4.5 \mathrm{mmol})$ in dry THF $(5 \mathrm{~mL})$ was added a solution of 1-II2 (411.7 mg, $3 \mathrm{mmol})$ in dry THF $(12 \mathrm{~mL})$ at $0{ }^{\circ} \mathrm{C}$ under a nitrogen atmosphere. The mixture was warmed up to room temperature and stirred for $2.5 \mathrm{~h}$. After TLC revealed the absence of the starting material, the reaction was quenched with cold water $(5 \mathrm{~mL})$ carefully at $0{ }^{\circ} \mathrm{C}$. Then the resulting mixture was filtered through Celite and the filtrate was extracted by $\mathrm{Et}_{2} \mathrm{O}(10 \mathrm{~mL} \times 3)$. The combined organic layers were washed with brine and dried over $\mathrm{Na}_{2} \mathrm{SO}_{4}$. Filtration and evaporation of the solvents left a crude mixture 1-II3, which was used directly for the next step.

To a solution of 1-II3 $(1.5 \mathrm{mmol})$ in dry DCM $(6 \mathrm{~mL})$ were subsequently added $\mathrm{Et}_{3} \mathrm{~N}(1.8 \mathrm{mmol})$ 
and $\mathrm{NsCl}(1.8 \mathrm{mmol})$ at $0{ }^{\circ} \mathrm{C}$. The resulting solution was stirred at room temperature overnight, after which it was treated with water $(6 \mathrm{~mL})$ and extracted with DCM $(6 \mathrm{~mL} \times 3)$. The combined organic layers were dried over $\mathrm{Na}_{2} \mathrm{SO}_{4}$ and concentrated in vacuo. The residue was purified by flash chromatography on silica gel to give product $\mathbf{1 k}$.

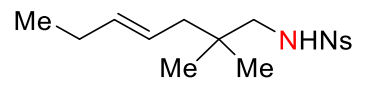

$N$-(2,2-Dimethylhept-4-en-1-yl)-4-nitrobenzenesulfonamide (1k). Flash column chromatography (eluent: PE/EtOAc $=5 / 1$, v/v) to afford $1 \mathbf{k}$ as a yellow solid, $377.0 \mathrm{mg}, 77 \%$ yield in the last step. ${ }^{1} \mathrm{H}$ NMR (400 MHz, $\left.\mathrm{CDCl}_{3}\right) \delta 8.40-8.35(\mathrm{~m}, 2 \mathrm{H}), 8.07-8.01(\mathrm{~m}, 2 \mathrm{H}), 5.50-5.26(\mathrm{~m}, 2 \mathrm{H}), 4.70(\mathrm{br}, \mathrm{s}$, $1 \mathrm{H}), 2.75(\mathrm{~d}, J=6.7 \mathrm{~Hz}, 2 \mathrm{H}), 1.98(\mathrm{p}, J=6.4 \mathrm{~Hz}, 2 \mathrm{H}), 1.89(\mathrm{dd}, J=7.3,0.8 \mathrm{~Hz}, 2 \mathrm{H}), 0.94(\mathrm{t}, J=7.5$ $\mathrm{Hz}, 3 \mathrm{H}), 0.86(\mathrm{~s}, 6 \mathrm{H}) .{ }^{13} \mathrm{C} \mathrm{NMR}\left(101 \mathrm{MHz}, \mathrm{CDCl}_{3}\right) \delta 150.16,146.12,136.18,128.39,124.54$, 124.20, 53.15, 42.91, 34.46, 25.80, 25.04, 14.04. HR-ESI-MS m/z calcd. for $\mathrm{C}_{15} \mathrm{H}_{21} \mathrm{~N}_{2} \mathrm{O}_{4} \mathrm{~S}[\mathrm{M}-\mathrm{H}]^{-}$: 325.1228, found: 325.1237 .

\subsection{The procedure for the preparation of sulfonamide $1 \mathrm{~s}$}

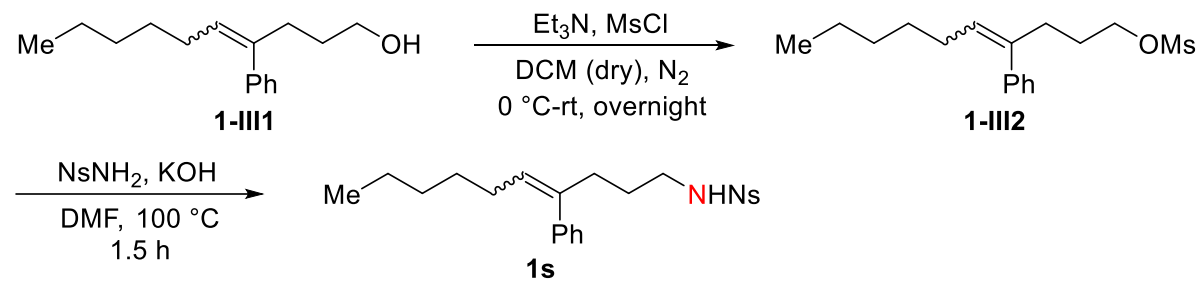

Sulfonamide 1r was synthesized according to the procedure in the literature, ${ }^{4 a}$ and 4-phenyldec4-en-1-ol was prepared according to the known procedure. ${ }^{6}$

To a solution of alcohol 1-III1 $(2.0 \mathrm{mmol})$ and triethylamine $(417 \mu \mathrm{L}, 3.0 \mathrm{mmol})$ in dry DCM $(10 \mathrm{~mL})$ at $0{ }^{\circ} \mathrm{C}$ was added $\mathrm{MsCl}(201 \mu \mathrm{L}, 2.6 \mathrm{mmol})$ under a nitrogen atmosphere. The resulting mixture was stirred at $0{ }^{\circ} \mathrm{C}$ for $10 \mathrm{~min}$, after which it was warmed up to room temperature and stirred overnight. Then the reaction was quenched with water $(10 \mathrm{~mL})$ and extracted with DCM (10 $\mathrm{mL} \times 3)$. The combined organic extracts were dried over anhydrous $\mathrm{Na}_{2} \mathrm{SO}_{4}$, filtered, and concentrated in vacuo. The residue was purified by flash column chromatography (eluent: $\mathrm{PE} / \mathrm{EA}=4: 1$ ) to give compound 1-III2.

A solution of $\mathrm{NsNH}_{2}(592.4 \mathrm{mg}, 2.9 \mathrm{mmol})$ and $\mathrm{KOH}(164.4 \mathrm{mg}, 2.9 \mathrm{mmol})$ in DMF $(9 \mathrm{~mL})$ was stirred at $100{ }^{\circ} \mathrm{C}$ for $0.5 \mathrm{~h}$. Then, to the resulting mixture was added a solution of compound 1-III2 (606.8 mg, $2.0 \mathrm{mmol}$ ) in DMF (3 mL) dropwise. After addition, the mixture was stirred at $100{ }^{\circ} \mathrm{C}$ for additional $1 \mathrm{~h}$, and then cooled to room temperature. The reaction mixture was diluted with water $(12 \mathrm{~mL})$ and extracted with $\mathrm{DCM}(12 \mathrm{~mL} \times 3)$. The combined organic extracts were 
washed with water $(12 \mathrm{~mL} \times 3)$ and brine $(12 \mathrm{~mL})$, dried over anhydrous $\mathrm{Na}_{2} \mathrm{SO}_{4}$, filtered, and concentrated in vacuo. The residue was purified by flash column chromatography to give sulfonamide 1 s.

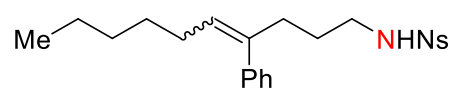

4-Nitro- $N$-(4-phenyldec-4-en-1-yl)benzenesulfonamide (1s). Flash column chromatography (eluent: PE/EtOAc $=20 / 1$ to 4/1, v/v) to afford $1 \mathrm{~s}$ as a yellow solid, $568.9 \mathrm{mg}, 69 \%$ yield in the last step, $E / Z=4.6: 1 .{ }^{1} \mathrm{H}$ NMR (400 MHz, $\mathrm{CDCl}_{3}$ ) Major: $\delta 8.34-8.24(\mathrm{~m}, 1.64 \mathrm{H}), 7.97(\mathrm{~d}, J=8.5 \mathrm{~Hz}$, $1.64 \mathrm{H}), 7.30-7.24(\mathrm{~m}, 2.46 \mathrm{H}), 7.03(\mathrm{~d}, J=7.5 \mathrm{~Hz}, 1.64 \mathrm{H}), 5.40(\mathrm{t}, J=7.1 \mathrm{~Hz}, 0.82 \mathrm{H}), 4.74-4.39$ $(\mathrm{m}, 0.82 \mathrm{H}), 3.02-2.94(\mathrm{~m}, 1.64 \mathrm{H}), 2.32(\mathrm{t}, J=7.1 \mathrm{~Hz}, 1.64 \mathrm{H}), 1.97-1.80(\mathrm{~m}, 1.64 \mathrm{H}), 1.58(\mathrm{~d}, J=$ $10.4 \mathrm{~Hz}, 0.82 \mathrm{H}), 1.51-1.36(\mathrm{~m}, 2.46 \mathrm{H}), 1.31-1.19(\mathrm{~m}, 3.28 \mathrm{H}), 0.83(\mathrm{t}, J=6.4 \mathrm{~Hz}, 2.46 \mathrm{H})$. Minor: $\delta 8.34-8.24(\mathrm{~m}, 0.36 \mathrm{H}), 7.91(\mathrm{~d}, J=8.4 \mathrm{~Hz}, 0.36 \mathrm{H}), 7.30-7.24(\mathrm{~m}, 0.72 \mathrm{H}), 7.13(\mathrm{~d}, J=7.4 \mathrm{~Hz}$, $0.18 \mathrm{H}), 5.66(\mathrm{t}, J=7.3 \mathrm{~Hz}, 0.18 \mathrm{H}), 4.74-4.39(\mathrm{~m}, 0.18 \mathrm{H}), 3.74-3.51(\mathrm{~m}, 0.18 \mathrm{H}), 3.02-2.94(\mathrm{~m}$, $0.36 \mathrm{H}), 2.51(\mathrm{t}, J=7.2 \mathrm{~Hz}, 0.36 \mathrm{H}), 2.12(\mathrm{q}, J=7.1 \mathrm{~Hz}, 0.36 \mathrm{H}), 1.97-1.80(\mathrm{~m}, 0.18 \mathrm{H}), 1.58(\mathrm{~d}, J=$ $10.4 \mathrm{~Hz}, 0.18 \mathrm{H}), 1.31-1.19(\mathrm{~m}, 0.90 \mathrm{H}), 0.92-0.88(\mathrm{~m}, 0.54 \mathrm{H}) .{ }^{13} \mathrm{C}$ NMR $\left(101 \mathrm{MHz}, \mathrm{CDCl}_{3}\right)$ Major: $\delta 150.13,146.26,140.42,138.95,129.23,128.44,128.35,128.31,126.86,124.48,43.04$, 36.22 , 31.53, 29.79, 28.91, 28.19, 22.60, 14.12. HR-ESI-MS $m / z$ calcd. for $\mathrm{C}_{22} \mathrm{H}_{27} \mathrm{~N}_{2} \mathrm{O}_{4} \mathrm{~S}[\mathrm{M}-\mathrm{H}]^{-}$: 415.1697, found: 415.1701 .

\subsection{General procedure for the preparation of sulfonamides 11 and $1 \mathrm{~m}$}

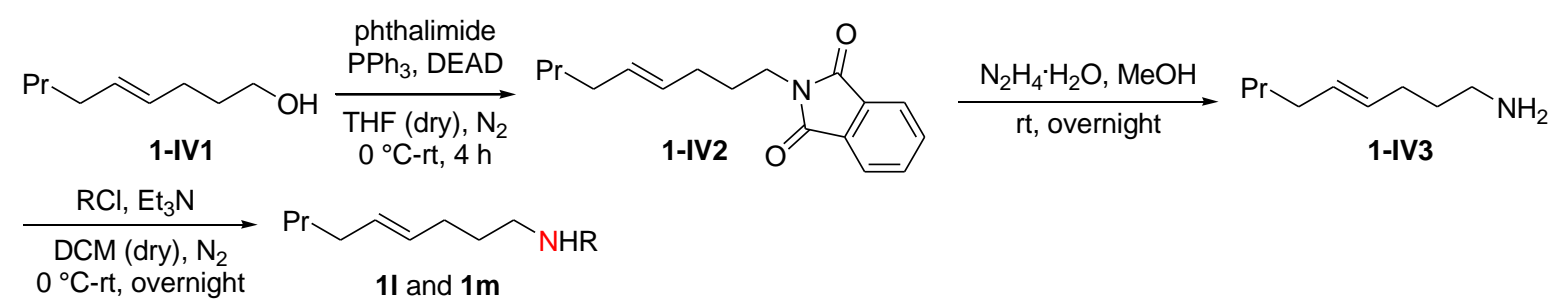

To a solution of triphenylphosphine $\left(\mathrm{PPh}_{3}, 1358.7 \mathrm{mg}, 5.2 \mathrm{mmol}\right)$ and phthalimide $(762.1 \mathrm{mg}$, $5.2 \mathrm{mmol})$ in dry THF $(25 \mathrm{~mL})$ at $0{ }^{\circ} \mathrm{C}$ were added alcohol 1-IV1 $(736.7 \mathrm{mg}, 5.2 \mathrm{mmol})$ and diethyl azodicarboxylate (DEAD, $816 \mu \mathrm{L}, 5.2 \mathrm{mmol}$ ) under a nitrogen atmosphere. The resulting solution was stirred at $0{ }^{\circ} \mathrm{C}$ for $10 \mathrm{~min}$, and then was stirred at room temperature for additional $4 \mathrm{~h}$. The reaction solution was quenched with water $(25 \mathrm{~mL})$ and extracted with DCM $(25 \mathrm{~mL} \times 3)$. The combined organic extracts were washed with brine $(25 \mathrm{~mL})$, dried over anhydrous $\mathrm{Na}_{2} \mathrm{SO}_{4}$, filtered, and concentrated in vасио. The residue was purified by flash column chromatography (eluent: $\mathrm{PE} / \mathrm{EA}=10: 1)$ to give compound 1-IV2.

A solution of compound 1-IV2 $(1271.6 \mathrm{mg}, 4.7 \mathrm{mmol})$ and $\mathrm{N}_{2} \mathrm{H}_{4} \cdot \mathrm{H}_{2} \mathrm{O}(914 \mu \mathrm{L}, 18.8 \mathrm{mmol})$ in 
$\mathrm{MeOH}(9 \mathrm{~mL})$ was stirred at room temperature for overnight. Upon completion of the reaction, it was acidified with $1 \mathrm{M} \mathrm{HCl}$ to $\mathrm{pH}=3$ and washed with $\mathrm{DCM}(10 \mathrm{~mL} \times 3)$. The aqueous phase was treated with $10 \% \mathrm{NaOH}$ solution to $\mathrm{pH}=12$, then extracted with $\mathrm{DCM}(10 \mathrm{~mL} \times 3)$. The combined organic extracts were washed with brine $(10 \mathrm{~mL})$, dried over anhydrous $\mathrm{Na}_{2} \mathrm{SO}_{4}$, filtered, and concentrated to give amine 1-IV3.

To a solution of amine 1-IV3 $(1.0 \mathrm{mmol})$ and triethylamine $(209 \mu \mathrm{L}, 1.5 \mathrm{mmol})$ in dry DCM (3 $\mathrm{mL})$ at $0{ }^{\circ} \mathrm{C}$ was added $\mathrm{RCl}(1.2 \mathrm{mmol})$ under a nitrogen atmosphere. The resulting solution was stirred at $0{ }^{\circ} \mathrm{C}$ for $10 \mathrm{~min}$, after which it was warmed up to room temperature and stirred overnight. Then the reaction was quenched with water $(3 \mathrm{~mL})$ and extracted with DCM $(3 \mathrm{~mL} \times 3)$. The combined organic extracts were dried over anhydrous $\mathrm{Na}_{2} \mathrm{SO}_{4}$, filtered, and concentrated in vacuo. The residue was purified by flash column chromatography to give the desired product.

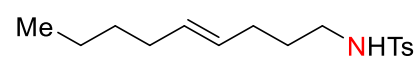

4-Methyl- $N$-(non-4-en-1-yl)benzenesulfonamide (11). Flash column chromatography (eluent: $\mathrm{PE} / \mathrm{EtOAc}=25 / 1$ to $10 / 1, \mathrm{v} / \mathrm{v})$ to afford 11 as a colorless oil, $387.6 \mathrm{mg}$, 97\% yield in the last step with $1.4 \mathrm{mmol}$ 1-IV3. ${ }^{1} \mathrm{H}$ NMR $\left(400 \mathrm{MHz}, \mathrm{CDCl}_{3}\right) \delta 7.75(\mathrm{~d}, J=8.3 \mathrm{~Hz}, 2 \mathrm{H}), 7.30(\mathrm{~d}, J=8.0 \mathrm{~Hz}$, 2H), $5.50-5.13(\mathrm{~m}, 2 \mathrm{H}), 4.68(\mathrm{br}, \mathrm{s}, 1 \mathrm{H}), 2.93(\mathrm{q}, J=6.8 \mathrm{~Hz}, 2 \mathrm{H}), 2.43(\mathrm{~s}, 3 \mathrm{H}), 2.02-1.88(\mathrm{~m}, 4 \mathrm{H})$, $1.51(\mathrm{p}, J=7.1 \mathrm{~Hz}, 2 \mathrm{H}), 1.32-1.24(\mathrm{~m}, 4 \mathrm{H}), 0.87(\mathrm{t}, J=7.0 \mathrm{~Hz}, 3 \mathrm{H}) .{ }^{13} \mathrm{C} \mathrm{NMR}\left(101 \mathrm{MHz}, \mathrm{CDCl}_{3}\right)$ $\delta 143.41,137.13,131.97,129.78,128.48,127.23,42.76,32.29,31.75,29.61,29.38,22.29,21.63$, 14.05. HR-ESI-MS $m / z$ calcd. for $\mathrm{C}_{16} \mathrm{H}_{25} \mathrm{NNaO}_{2} \mathrm{~S}[\mathrm{M}+\mathrm{Na}]^{+}: 318.1498$, found: 318.1490 .

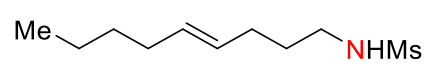

$N$-(Non-4-en-1-yl)methanesulfonamide (1m). Flash column chromatography (eluent: PE/EtOAc = $15 / 1$ to $3 / 1, \mathrm{v} / \mathrm{v}$ ) to afford $\mathbf{1 m}$ as a colorless oil, $210.6 \mathrm{mg}, 96 \%$ yield in the last step. ${ }^{1} \mathrm{H}$ NMR (400 $\left.\mathrm{MHz}, \mathrm{CDCl}_{3}\right) \delta 5.58-5.27(\mathrm{~m}, 2 \mathrm{H}), 4.48(\mathrm{br}, \mathrm{s}, 1 \mathrm{H}), 3.13(\mathrm{dd}, J=13.5,6.8 \mathrm{~Hz}, 2 \mathrm{H}), 2.95(\mathrm{~s}, 3 \mathrm{H})$, $2.07(\mathrm{q}, J=6.8 \mathrm{~Hz}, 2 \mathrm{H}), 1.99(\mathrm{q}, J=6.5 \mathrm{~Hz}, 2 \mathrm{H}), 1.67-1.61(\mathrm{~m}, 2 \mathrm{H}), 1.35-1.27(\mathrm{~m}, 4 \mathrm{H}), 0.92-$ $0.86(\mathrm{~m}, 3 \mathrm{H}) .{ }^{13} \mathrm{C} \mathrm{NMR}\left(101 \mathrm{MHz}, \mathrm{CDCl}_{3}\right) \delta 132.18,128.43,42.89,40.37,32.33,31.78,29.95$, 29.66, 22.31, 14.05. HR-ESI-MS $m / z$ calcd. for $\mathrm{C}_{16} \mathrm{H}_{25} \mathrm{NNaO}_{2} \mathrm{~S}\left[\mathrm{M}+\mathrm{Na}^{+}\right.$: 318.1498 , found: 318.1490. HR-ESI-MS $m / z$ calcd. for $\mathrm{C}_{10} \mathrm{H}_{21} \mathrm{NNaO}_{2} \mathrm{~S}[\mathrm{M}+\mathrm{Na}]^{+}: 242.1185$, found: 242.1183 .

\section{General Procedure for the Synthesis of Saturated}

\section{Thiocyano-Containing Heterocycles by Selenide Catalysis}

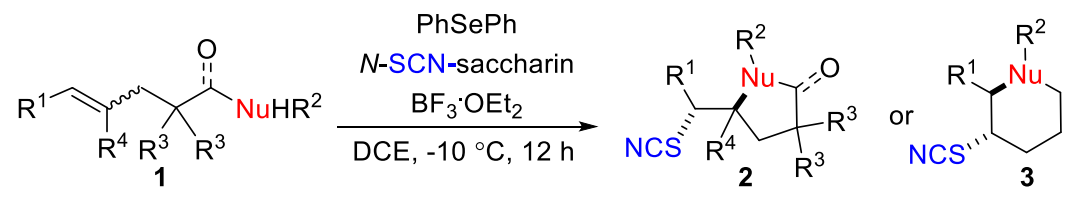


In a $10 \mathrm{~mL}$ Schlenk flask were subsequently added substrate $1(0.10 \mathrm{mmol})$, DCE $(2.0 \mathrm{~mL})$, $\mathrm{PhSePh}(3.5 \mu \mathrm{L}, 20 \mathrm{~mol} \%)$, NTSc (36.0 mg, 1.5 equiv), and $\mathrm{BF}_{3} \cdot \mathrm{OEt}_{2}$ (24.7 $\mu \mathrm{L}, 2.0$ equiv) in the dark. The solution was stirred in the dark at $-10{ }^{\circ} \mathrm{C}$ for $12 \mathrm{~h}$. Then it was quenched with $\mathrm{Et}_{3} \mathrm{~N}$ (55.6 $\mu \mathrm{L}, 0.40 \mathrm{mmol}$ ) and concentrated in vacuo. The residue was purified by silica gel column chromatography to afford the corresponding saturated thiocyano-containing heterocycles $\mathbf{2}$ or $\mathbf{3}$.

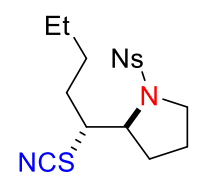

1-((4-Nitrophenyl)sulfonyl)-2-(1-thiocyanatopentyl)pyrrolidine (2a). Flash column chromatography (eluent: $\mathrm{PE} / \mathrm{EtOAc}=20 / 1$ to $6 / 1, \mathrm{v} / \mathrm{v}$ ) to afford $\mathbf{2 a}$ as a yellow solid, $34.8 \mathrm{mg}, 91 \%$ yield. ${ }^{1} \mathrm{H}$ NMR (400 MHz, $\left.\mathrm{CDCl}_{3}\right) \delta 8.45-8.39(\mathrm{~m}, 2 \mathrm{H}), 8.07-8.01(\mathrm{~m}, 2 \mathrm{H}), 3.95-3.89(\mathrm{~m}, 1 \mathrm{H})$, $3.84-3.77(\mathrm{~m}, 1 \mathrm{H}), 3.52-3.39(\mathrm{~m}, 2 \mathrm{H}), 2.06-1.96(\mathrm{~m}, 2 \mathrm{H}), 1.88-1.75(\mathrm{~m}, 3 \mathrm{H}), 1.69-1.60(\mathrm{~m}$, $1 \mathrm{H}), 1.52-1.39(\mathrm{~m}, 4 \mathrm{H}), 0.97(\mathrm{t}, J=7.2 \mathrm{~Hz}, 3 \mathrm{H}) .{ }^{13} \mathrm{C} \mathrm{NMR}\left(101 \mathrm{MHz}, \mathrm{CDCl}_{3}\right) \delta 150.47,143.08$, $128.85,124.69,111.32,77.48,77.16,76.84,63.82,55.12,50.15,32.15,29.39,27.02,24.84,22.32$, 13.93. HR-ESI-MS $m / z$ calcd. for $\mathrm{C}_{16} \mathrm{H}_{21} \mathrm{~N}_{3} \mathrm{NaO}_{4} \mathrm{~S}_{2}[\mathrm{M}+\mathrm{Na}]^{+}:$406.0866, found: 406.0869 .

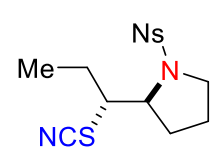

1-((4-Nitrophenyl)sulfonyl)-2-(1-thiocyanatopropyl)pyrrolidine $\quad$ (2b). Flash column chromatography (eluent: $\mathrm{PE} / \mathrm{EtOAc}=15 / 1$ to $6 / 1, \mathrm{v} / \mathrm{v}$ ) to afford $\mathbf{2 b}$ as a colorless oil, $33.4 \mathrm{mg}, 94 \%$ yield. ${ }^{1} \mathrm{H}$ NMR $\left(400 \mathrm{MHz}, \mathrm{CDCl}_{3}\right) \delta 8.41(\mathrm{~d}, J=8.7 \mathrm{~Hz}, 2 \mathrm{H}), 8.04(\mathrm{~d}, J=8.7 \mathrm{~Hz}, 2 \mathrm{H}), 3.97-3.85$ $(\mathrm{m}, 1 \mathrm{H}), 3.71(\mathrm{p}, J=9.4,4.7 \mathrm{~Hz}, 1 \mathrm{H}), 3.56-3.37(\mathrm{~m}, 2 \mathrm{H}), 2.04-1.75(\mathrm{~m}, 5 \mathrm{H}), 1.54-1.44(\mathrm{~m}, 1 \mathrm{H})$, $1.20(\mathrm{t}, J=7.3 \mathrm{~Hz}, 3 \mathrm{H}) .{ }^{13} \mathrm{C} \mathrm{NMR}\left(101 \mathrm{MHz}, \mathrm{CDCl}_{3}\right) \delta 150.48,143.05,128.86,124.69,111.22$, $63.77,57.03,50.11,27.11,25.82,24.81,12.15$. HR-ESI-MS $m / z$ calcd. for $\mathrm{C}_{14} \mathrm{H}_{17} \mathrm{~N}_{3} \mathrm{NaO}_{4} \mathrm{~S}_{2}[\mathrm{M}+$ $\mathrm{Na}]^{+}:$378.0553, found: 378.0542 .

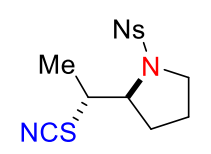

rel-(S)-1-((4-Nitrophenyl)sulfonyl)-2-((R)-1-thiocyanatoethyl)pyrrolidine (2c). Flash column chromatography (eluent: PE/EtOAc $=15 / 1$ to 5/1, v/v) to afford $2 \mathrm{c}$ as a yellow solid, $27.3 \mathrm{mg}, 80 \%$ yield. ${ }^{1} \mathrm{H}$ NMR $\left(400 \mathrm{MHz}, \mathrm{CDCl}_{3}\right) \delta 8.41(\mathrm{~d}, J=8.7 \mathrm{~Hz}, 2 \mathrm{H}), 8.05(\mathrm{~d}, J=8.7 \mathrm{~Hz}, 2 \mathrm{H}), 4.06-3.96$ $(\mathrm{m}, 1 \mathrm{H}), 3.90-3.84(\mathrm{~m}, 1 \mathrm{H}), 3.50-3.37(\mathrm{~m}, 2 \mathrm{H}), 2.03-1.94(\mathrm{~m}, 2 \mathrm{H}), 1.89-1.82(\mathrm{~m}, 1 \mathrm{H}), 1.58(\mathrm{~d}$, $J=7.1 \mathrm{~Hz}, 3 \mathrm{H}), 1.52-1.46(\mathrm{~m}, 1 \mathrm{H}) .{ }^{13} \mathrm{C} \mathrm{NMR}\left(101 \mathrm{MHz}, \mathrm{CDCl}_{3}\right) \delta 150.53,143.05,128.92,124.72$, 
111.15, 64.51, 50.14, 49.14, 27.12, 24.75, 18.88. HR-ESI-MS $m / z$ calcd. for $\mathrm{C}_{13} \mathrm{H}_{15} \mathrm{~N}_{3} \mathrm{NaO}_{4} \mathrm{~S}_{2}[\mathrm{M}+$ $\mathrm{Na}]^{+}:$364.0396, found: 364.0391 .

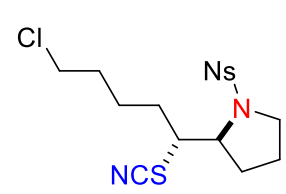

2-(5-Chloro-1-thiocyanatopentyl)-1-((4-nitrophenyl)sulfonyl)pyrrolidine (2d). Flash column chromatography (eluent: $\mathrm{PE} / \mathrm{EtOAc}=15 / 1$ to $4 / 1, \mathrm{v} / \mathrm{v}$ ) to afford $\mathbf{2 d}$ as a yellow oil, $37.7 \mathrm{mg}, 90 \%$ yield. ${ }^{1} \mathrm{H}$ NMR $\left(400 \mathrm{MHz}, \mathrm{CDCl}_{3}\right) \delta 8.48-8.38(\mathrm{~m}, 2 \mathrm{H}), 8.07-8.02(\mathrm{~m}, 2 \mathrm{H}), 3.94-3.89(\mathrm{~m}, 1 \mathrm{H})$, $3.83-3.76(\mathrm{~m}, 1 \mathrm{H}), 3.61(\mathrm{t}, J=6.1 \mathrm{~Hz}, 2 \mathrm{H}), 3.53-3.38(\mathrm{~m}, 2 \mathrm{H}), 2.04-1.96(\mathrm{~m}, 2 \mathrm{H}), 1.94-1.79$ $(\mathrm{m}, 6 \mathrm{H}), 1.71-1.65(\mathrm{~m}, 1 \mathrm{H}), 1.53-1.46(\mathrm{~m}, 1 \mathrm{H}) .{ }^{13} \mathrm{C} \mathrm{NMR}\left(101 \mathrm{MHz}, \mathrm{CDCl}_{3}\right) \delta 150.53,142.94$, 128.90, 124.73, 111.05, 63.67, 54.78, 50.13, 44.60, 31.86, 31.73, 27.13, 24.79, 24.66. HR-ESI-MS $m / z$ calcd. for $\mathrm{C}_{16} \mathrm{H}_{20} \mathrm{ClN}_{3} \mathrm{NaO}_{4} \mathrm{~S}_{2}[\mathrm{M}+\mathrm{Na}]^{+}:$440.0476, found: 440.0489 .

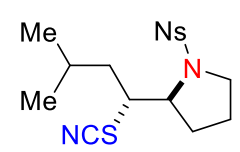

2-(3-Methyl-1-thiocyanatobutyl)-1-((4-nitrophenyl)sulfonyl)pyrrolidine (2e). Flash column chromatography (eluent: $\mathrm{PE} / \mathrm{EtOAc}=20 / 1$ to $5 / 1$, v/v) to afford $2 \mathrm{e}$ as a yellow solid, $37.8 \mathrm{mg}, 99 \%$ yield. ${ }^{1} \mathrm{H}$ NMR (400 MHz, $\left.\mathrm{CDCl}_{3}\right) \delta 8.48-8.37(\mathrm{~m}, 2 \mathrm{H}), 8.06-8.00(\mathrm{~m}, 2 \mathrm{H}), 3.95-3.86(\mathrm{~m}, 2 \mathrm{H})$, $3.53-3.40(\mathrm{~m}, 2 \mathrm{H}), 2.06-1.97(\mathrm{~m}, 2 \mathrm{H}), 1.92-1.80(\mathrm{~m}, 2 \mathrm{H}), 1.76-1.68(\mathrm{~m}, 1 \mathrm{H}), 1.62-1.56(\mathrm{~m}$, $1 \mathrm{H}), 1.53-1.44(\mathrm{~m}, 1 \mathrm{H}), 1.07(\mathrm{~d}, J=6.6 \mathrm{~Hz}, 3 \mathrm{H}), 1.01(\mathrm{~d}, J=6.5 \mathrm{~Hz}, 3 \mathrm{H}) .{ }^{13} \mathrm{C} \mathrm{NMR}(101 \mathrm{MHz}$, $\left.\mathrm{CDCl}_{3}\right) \delta 150.50,143.13,128.86,124.71,111.37,63.77,53.03,50.18,41.32,26.96,25.84,24.90$, 22.71, 22.00. HR-ESI-MS $m / z$ calcd. for $\mathrm{C}_{16} \mathrm{H}_{21} \mathrm{~N}_{3} \mathrm{NaO}_{4} \mathrm{~S}_{2}[\mathrm{M}+\mathrm{Na}]^{+}:$406.0866, found: 406.0875 .

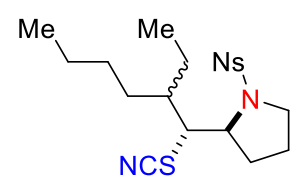

2-(2-Ethyl-1-thiocyanatohexyl)-1-((4-nitrophenyl)sulfonyl)pyrrolidine (2f). Flash column chromatography (eluent: $\mathrm{PE} / \mathrm{EtOAc}=20 / 1$ to $8 / 1, \mathrm{v} / \mathrm{v}$ ) to afford $\mathbf{2 f}$ as a yellow oil, $29.9 \mathrm{mg}, 70 \%$ yield, $d r=1: 1 .{ }^{1} \mathrm{H}$ NMR $\left(400 \mathrm{MHz}, \mathrm{CDCl}_{3}\right) \delta 8.41(\mathrm{~d}, J=9.2 \mathrm{~Hz}, 1 \mathrm{H}), 8.40(\mathrm{~d}, J=8.8 \mathrm{~Hz}, 1 \mathrm{H}), 8.04$ $(\mathrm{d}, J=8.8 \mathrm{~Hz}, 1 \mathrm{H}), 8.03(\mathrm{~d}, J=8.8 \mathrm{~Hz}, 1 \mathrm{H}), 4.14-4.06(\mathrm{~m}, 1 \mathrm{H}), 3.84-3.79(\mathrm{~m}, 1 \mathrm{H}), 3.49-3.45$ $(\mathrm{m}, 2 \mathrm{H}), 2.07-1.98(\mathrm{~m}, 2 \mathrm{H}), 1.86-1.78(\mathrm{~m}, 2 \mathrm{H}), 1.74-1.64(\mathrm{~m}, 1 \mathrm{H}), 1.57-1.34(\mathrm{~m}, 8 \mathrm{H}), 1.02-$ $0.94(\mathrm{~m}, 6 \mathrm{H}) .{ }^{13} \mathrm{C} \mathrm{NMR}\left(101 \mathrm{MHz}, \mathrm{CDCl}_{3}\right) \delta 150.51,143.46,128.85,124.72,124.70,112.20,62.07$, 62.04, 59.79, 59.64, 49.94, 49.89, 41.29, 41.18, 29.73, 29.44, 28.61, 27.95, 27.72, 27.53, 25.08, 25.00, 23.24, 23.12, 22.98, 22.75, 14.15, 10.77, 9.92. HR-ESI-MS $m / z$ calcd. for $\mathrm{C}_{19} \mathrm{H}_{27} \mathrm{~N}_{3} \mathrm{NaO}_{4} \mathrm{~S}_{2}$ $[\mathrm{M}+\mathrm{Na}]^{+}:$448.1335, found: 448.1326. 


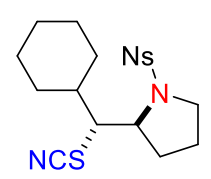

2-(Cyclohexyl(thiocyanato)methyl)-1-((4-nitrophenyl)sulfonyl)pyrrolidine (2g). Flash column chromatography (eluent: $\mathrm{PE} / \mathrm{EtOAc}=20 / 1$ to $8 / 1, \mathrm{v} / \mathrm{v}$ ) to afford $\mathbf{2 g}$ as a yellow solid, $38.4 \mathrm{mg}, 94 \%$ yield. ${ }^{1} \mathrm{H}$ NMR $\left(400 \mathrm{MHz}, \mathrm{CDCl}_{3}\right) \delta 8.45-8.37(\mathrm{~m}, 2 \mathrm{H}), 8.06-8.00(\mathrm{~m}, 2 \mathrm{H}), 4.17-4.11(\mathrm{~m}, 1 \mathrm{H})$, $3.68(\mathrm{dd}, J=8.9,3.8 \mathrm{~Hz}, 1 \mathrm{H}), 3.53-3.42(\mathrm{~m}, 2 \mathrm{H}), 2.18(\mathrm{~d}, J=12.6 \mathrm{~Hz}, 1 \mathrm{H}), 2.05-1.96(\mathrm{~m}, 2 \mathrm{H})$, $1.88-1.79(\mathrm{~m}, 4 \mathrm{H}), 1.77-1.68(\mathrm{~m}, 2 \mathrm{H}), 1.52-1.44(\mathrm{~m}, 1 \mathrm{H}), 1.37-1.25(\mathrm{~m}, 4 \mathrm{H}), 1.22-1.16(\mathrm{~m}$, 1H). ${ }^{13} \mathrm{C}$ NMR $\left(101 \mathrm{MHz}, \mathrm{CDCl}_{3}\right) \delta 150.48,143.23,128.84,124.72,112.11,61.93,61.52,50.10$, $39.90,31.31,31.17,27.15,26.02,25.94,25.72$, 24.98. HR-ESI-MS $m / z$ calcd. for $\mathrm{C}_{18} \mathrm{H}_{23} \mathrm{~N}_{3} \mathrm{NaO}_{4} \mathrm{~S}_{2}$ $[\mathrm{M}+\mathrm{Na}]^{+}:$432.1022, found: 432.1024 .

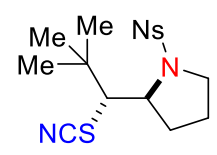

2-(2,2-Dimethyl-1-thiocyanatopropyl)-1-((4-nitrophenyl)sulfonyl)pyrrolidine (2h). Flash column chromatography (eluent: PE/EtOAc $=25 / 1$ to $12 / 1$, v/v) to afford $\mathbf{2 h}$ as a yellow solid, $19.6 \mathrm{mg}, 51 \%$ yield. ${ }^{1} \mathrm{H}$ NMR (400 MHz, $\left.\mathrm{CDCl}_{3}\right) \delta 8.43-8.39(\mathrm{~m}, 2 \mathrm{H}), 8.13-8.07(\mathrm{~m}, 2 \mathrm{H}), 4.39-4.34(\mathrm{~m}, 1 \mathrm{H})$, $4.09(\mathrm{~d}, J=2.0 \mathrm{~Hz}, 1 \mathrm{H}), 3.51-3.46(\mathrm{~m}, 1 \mathrm{H}), 3.38-3.32(\mathrm{~m}, 1 \mathrm{H}), 2.07-1.97(\mathrm{~m}, 2 \mathrm{H}), 1.82-1.76$ $(\mathrm{m}, 1 \mathrm{H}), 1.56-1.49(\mathrm{~m}, 1 \mathrm{H}), 1.16(\mathrm{~s}, 9 \mathrm{H}) .{ }^{13} \mathrm{C} \mathrm{NMR}\left(101 \mathrm{MHz}, \mathrm{CDCl}_{3}\right) \delta 150.52,143.74,129.10$, 124.69, 113.03, 70.12, 60.42, 49.47, 36.17, 29.42, 28.50, 25.99. HR-ESI-MS m/z calcd. for $\mathrm{C}_{16} \mathrm{H}_{21} \mathrm{~N}_{3} \mathrm{NaO}_{4} \mathrm{~S}_{2}[\mathrm{M}+\mathrm{Na}]^{+}:$406.0866, found: 406.0863 .

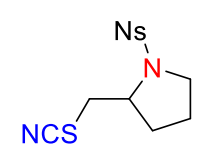

1-((4-Nitrophenyl)sulfonyl)-2-(thiocyanatomethyl)pyrrolidine (2i). Flash column chromatography (eluent: $\mathrm{PE} / \mathrm{EtOAc}=15 / 1$ to $3 / 1$, v/v) to afford $2 \mathbf{i}$ as a white solid, $32.7 \mathrm{mg}, 99 \%$ yield. ${ }^{1} \mathrm{H}$ NMR $\left(400 \mathrm{MHz} \mathrm{CDCl}_{3}\right) \delta 8.50-8.38(\mathrm{~m}, 2 \mathrm{H}), 8.09-8.05(\mathrm{~m}, 2 \mathrm{H}), 3.91-3.85(\mathrm{~m}, 1 \mathrm{H}), 3.62-3.54(\mathrm{~m}$, 2H), $3.25-3.19(\mathrm{~m}, 1 \mathrm{H}), 3.09-3.02(\mathrm{~m}, 1 \mathrm{H}), 2.01-1.90(\mathrm{~m}, 3 \mathrm{H}), 1.70-1.63(\mathrm{~m}, 1 \mathrm{H}) .{ }^{13} \mathrm{C}$ NMR $\left(101 \mathrm{MHz}, \mathrm{CDCl}_{3}\right) \delta 150.60,142.35,129.00,124.77,111.84,60.01,50.05,38.59,30.52,24.11$. HR-ESI-MS $m / z$ calcd. for $\mathrm{C}_{12} \mathrm{H}_{13} \mathrm{~N}_{3} \mathrm{NaO}_{4} \mathrm{~S}_{2}[\mathrm{M}+\mathrm{Na}]^{+}: 350.0240$, found: 350.0240 .

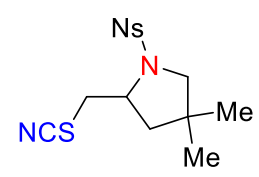

4,4-Dimethyl-1-((4-nitrophenyl)sulfonyl)-2-(thiocyanatomethyl)pyrrolidine $(2 \mathbf{j})$. Flash column chromatography (eluent: $\mathrm{PE} / \mathrm{EtOAc}=20 / 1$ to $5 / 1, \mathrm{v} / \mathrm{v}$ ) to afford $\mathbf{2} \mathbf{j}$ as a yellow oil, $33.8 \mathrm{mg}, 95 \%$ 
yield. ${ }^{1} \mathrm{H}$ NMR $\left(400 \mathrm{MHz}, \mathrm{CDCl}_{3}\right) \delta 8.42(\mathrm{~d}, J=8.8 \mathrm{~Hz}, 2 \mathrm{H}), 8.06(\mathrm{~d}, J=8.8 \mathrm{~Hz}, 2 \mathrm{H}), 3.94(\mathrm{~m}, 1 \mathrm{H})$, $3.72(\mathrm{dd}, J=13.4,2.7 \mathrm{~Hz}, 1 \mathrm{H}), 3.31-3.14(\mathrm{~m}, 3 \mathrm{H}), 2.04-1.96(\mathrm{~m}, 1 \mathrm{H}), 1.81-1.74(\mathrm{~m}, 1 \mathrm{H}), 1.11$ $(\mathrm{s}, 3 \mathrm{H}), 0.53(\mathrm{~s}, 3 \mathrm{H}) .{ }^{13} \mathrm{C}$ NMR $\left(101 \mathrm{MHz}, \mathrm{CDCl}_{3}\right) \delta 150.48,142.69,128.90,124.69,112.04,62.11$, 59.96, 45.20, 39.32, 37.96, 25.98, 25.73. HR-ESI-MS $\mathrm{m} / z$ calcd. for $\mathrm{C}_{14} \mathrm{H}_{17} \mathrm{~N}_{3} \mathrm{NaO}_{4} \mathrm{~S}_{2}[\mathrm{M}+\mathrm{Na}]^{+}$: 378.0553, found: 378.0556 .

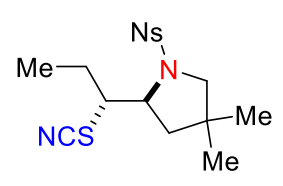

4,4-Dimethyl-1-((4-nitrophenyl)sulfonyl)-2-(1-thiocyanatopropyl)pyrrolidine (2k). Flash column chromatography (eluent: PE/EtOAc $=20 / 1$ to $6 / 1, \mathrm{v} / \mathrm{v}$ ) to afford $\mathbf{2 k}$ as a yellow solid, $36.0 \mathrm{mg}, 94 \%$ yield. ${ }^{1} \mathrm{H}$ NMR (400 MHz, $\left.\mathrm{CDCl}_{3}\right) \delta 8.40(\mathrm{~d}, J=8.8 \mathrm{~Hz}, 2 \mathrm{H}), 8.03(\mathrm{~d}, J=8.9 \mathrm{~Hz}, 2 \mathrm{H}), 4.14-4.05$ (m, 1H), $4.02-3.92(\mathrm{~m}, 1 \mathrm{H}), 3.33(\mathrm{~d}, J=11.0 \mathrm{~Hz}, 1 \mathrm{H}), 3.20(\mathrm{~d}, J=11.0 \mathrm{~Hz}, 1 \mathrm{H}), 1.84-1.68(\mathrm{~m}$, $4 \mathrm{H}), 1.18(\mathrm{t}, J=7.3 \mathrm{~Hz}, 3 \mathrm{H}), 1.10(\mathrm{~s}, 3 \mathrm{H}), 0.57(\mathrm{~s}, 3 \mathrm{H}) .{ }^{13} \mathrm{C} \mathrm{NMR}\left(101 \mathrm{MHz}, \mathrm{CDCl}_{3}\right) \delta 150.36$, $144.35,128.52,124.68,111.39,63.43,61.78,56.95,40.45,38.13,25.77,25.75,25.58,12.23$. HR-ESI-MS $m / z$ calcd. for $\mathrm{C}_{16} \mathrm{H}_{21} \mathrm{~N}_{3} \mathrm{NaO}_{4} \mathrm{~S}_{2}[\mathrm{M}+\mathrm{Na}]^{+}: 406.0866$, found: 406.0870 .

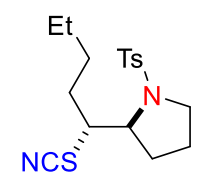

2-(1-Thiocyanatopentyl)-1-tosylpyrrolidine (2l). Flash column chromatography (eluent: PE/EtOAc $=20 / 1$ to $3 / 1, \mathrm{v} / \mathrm{v})$ to afford $2 \mathrm{l}$ as a colorless oil, $26.4 \mathrm{mg}, 75 \%$ yield. ${ }^{1} \mathrm{H}$ NMR $\left(400 \mathrm{MHz}, \mathrm{CDCl}_{3}\right) \delta$ $7.71(\mathrm{~d}, J=8.2 \mathrm{~Hz}, 2 \mathrm{H}), 7.34(\mathrm{~d}, J=8.1 \mathrm{~Hz}, 2 \mathrm{H}), 3.89-3.76(\mathrm{~m}, 2 \mathrm{H}), 3.45-3.34(\mathrm{~m}, 2 \mathrm{H}), 2.44$ (s, $3 \mathrm{H}), 2.02-1.86(\mathrm{~m}, 2 \mathrm{H}), 1.84-1.72(\mathrm{~m}, 3 \mathrm{H}), 1.67-1.61(\mathrm{~m}, 1 \mathrm{H}), 1.49-1.37(\mathrm{~m}, 4 \mathrm{H}), 0.96(\mathrm{t}, J=$ $7.1 \mathrm{~Hz}, 3 \mathrm{H}) .{ }^{13} \mathrm{C}$ NMR $\left(101 \mathrm{MHz}, \mathrm{CDCl}_{3}\right) \delta 144.20,134.27,130.06,127.73,111.82,63.47,55.23$, 50.07, 32.23, 29.45, 26.96, 24.76, 22.37, 21.70, 13.98. HR-ESI-MS $m / z$ calcd. for $\mathrm{C}_{17} \mathrm{H}_{24} \mathrm{~N}_{2} \mathrm{NaO}_{2} \mathrm{~S}_{2}$ $[\mathrm{M}+\mathrm{Na}]^{+}:$375.1171, found: 375.1170 .

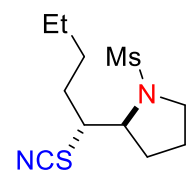

1-(Methylsulfonyl)-2-(1-thiocyanatopentyl)pyrrolidine (2m). Flash column chromatography (eluent: $\mathrm{PE} / \mathrm{EtOAc}=15 / 1$ to $3 / 1, \mathrm{v} / \mathrm{v}$ ) to afford $\mathbf{2 m}$ as a colorless oil, $23.1 \mathrm{mg}, 84 \%$ yield. ${ }^{1} \mathrm{H}$ NMR $\left(400 \mathrm{MHz}, \mathrm{CDCl}_{3}\right) \delta 4.12-4.01(\mathrm{~m}, 1 \mathrm{H}), 3.91-3.70(\mathrm{~m}, 1 \mathrm{H}), 3.57-3.47(\mathrm{~m}, 1 \mathrm{H}), 3.47-3.36(\mathrm{~m}$, $1 \mathrm{H}), 2.92(\mathrm{~s}, 3 \mathrm{H}), 2.16-1.96(\mathrm{~m}, 3 \mathrm{H}), 1.93-1.82(\mathrm{~m}, 1 \mathrm{H}), 1.73-1.59(\mathrm{~m}, 3 \mathrm{H}), 1.47-1.34(\mathrm{~m}, 3 \mathrm{H})$, $0.94(\mathrm{t}, J=7.2 \mathrm{~Hz}, 3 \mathrm{H}) .{ }^{13} \mathrm{C} \mathrm{NMR}\left(101 \mathrm{MHz}, \mathrm{CDCl}_{3}\right) \delta 112.10,63.12,55.66,49.77,37.11,31.97$, 
29.41, 26.98, 25.56, 22.33, 13.91. HR-ESI-MS $m / z$ calcd. for $\mathrm{C}_{11} \mathrm{H}_{20} \mathrm{~N}_{2} \mathrm{NaO}_{2} \mathrm{~S}_{2}[\mathrm{M}+\mathrm{Na}]^{+}:$299.0858, found: 299.0857.

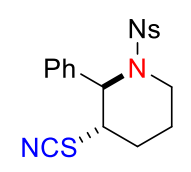

1-((4-Nitrophenyl)sulfonyl)-2-phenyl-3-thiocyanatopiperidine (3n). Flash column chromatography (eluent: PE/EtOAc $=20 / 1$ to 4/1, v/v) to afford 3n as a colorless oil, $40.0 \mathrm{mg}, 99 \%$ yield. ${ }^{1} \mathrm{H}$ NMR $\left(400 \mathrm{MHz}, \mathrm{CDCl}_{3}\right) \delta 8.33-8.26(\mathrm{~m}, 2 \mathrm{H}), 7.97-7.91(\mathrm{~m}, 2 \mathrm{H}), 7.35-7.29(\mathrm{~m}, 3 \mathrm{H})$, $7.20-7.16(\mathrm{~m}, 2 \mathrm{H}), 5.39$ (s, 1H), $4.31(\mathrm{dd}, J=6.1,3.2 \mathrm{~Hz}, 1 \mathrm{H}), 3.95(\mathrm{dd}, J=13.7,1.2 \mathrm{~Hz}, 1 \mathrm{H}), 3.40$ - $3.32(\mathrm{~m}, 1 \mathrm{H}), 2.04-1.92(\mathrm{~m}, 2 \mathrm{H}), 1.86-1.75(\mathrm{~m}, 1 \mathrm{H}), 1.68-1.61(\mathrm{~m}, 1 \mathrm{H}) .{ }^{13} \mathrm{C} \mathrm{NMR}(101 \mathrm{MHz}$, $\left.\mathrm{CDCl}_{3}\right) \delta 150.11,145.94,135.88,129.35,128.53,128.45,126.90,124.38,111.27,60.55,50.62$, 42.45, 24.07, 19.84. HR-ESI-MS $m / z$ calcd. for $\mathrm{C}_{18} \mathrm{H}_{17} \mathrm{~N}_{3} \mathrm{NaO}_{4} \mathrm{~S}_{2}[\mathrm{M}+\mathrm{Na}]^{+}: 426.0553$, found: 426.0559 .<smiles>Cc1ccc(C2[C@H](C#N)CCCN2N)cc1</smiles>

1-((4-Nitrophenyl)sulfonyl)-3-thiocyanato-2-( $p$-tolyl)piperidine (3o). Flash column chromatography (eluent: $\mathrm{PE} / \mathrm{EtOAc}=15 / 1$ to $3 / 1, \mathrm{v} / \mathrm{v}$ ) to afford 30 as a yellow oil, $41.5 \mathrm{mg}, 99 \%$ yield. ${ }^{1} \mathrm{H}$ NMR $\left(400 \mathrm{MHz}, \mathrm{CDCl}_{3}\right) \delta 8.32-8.24(\mathrm{~m}, 2 \mathrm{H}), 7.95-7.90(\mathrm{~m}, 2 \mathrm{H}), 7.11(\mathrm{~d}, J=8.1 \mathrm{~Hz}$, $2 \mathrm{H}), 7.04(\mathrm{~d}, J=8.2 \mathrm{~Hz}, 2 \mathrm{H}), 5.33(\mathrm{~s}, 1 \mathrm{H}), 4.27(\mathrm{dd}, J=6.4,3.3 \mathrm{~Hz}, 1 \mathrm{H}), 3.92(\mathrm{~d}, J=13.6 \mathrm{~Hz}, 1 \mathrm{H})$, $3.40-3.32(\mathrm{~m}, 1 \mathrm{H}), 2.32(\mathrm{~s}, 3 \mathrm{H}), 2.05-1.92(\mathrm{~m}, 2 \mathrm{H}), 1.87-1.77(\mathrm{~m}, 1 \mathrm{H}), 1.67-1.61(\mathrm{~m}, 1 \mathrm{H}) .{ }^{13} \mathrm{C}$ NMR $\left(101 \mathrm{MHz} \mathrm{CDCl}_{3}\right) \delta 150.06,145.99,138.54,132.78,129.94,128.44,126.88,124.32,111.33$, $60.47,50.63,42.45,24.15,21.05,19.95$. HR-ESI-MS $m / z$ calcd. for $\mathrm{C}_{19} \mathrm{H}_{19} \mathrm{~N}_{3} \mathrm{NaO}_{4} \mathrm{~S}_{2}[\mathrm{M}+\mathrm{Na}]^{+}$: 440.0709, found: 440.0705 .<smiles>N#C[C@@H]1CCCN([NH])C1c1ccc(Cl)cc1</smiles>

2-(4-Chlorophenyl)-1-((4-nitrophenyl)sulfonyl)-3-thiocyanatopiperidine (3p). Flash column chromatography (eluent: $\mathrm{PE} / \mathrm{EtOAc}=15 / 1$ to $3 / 1, \mathrm{v} / \mathrm{v}$ ) to afford $\mathbf{3 p}$ as a yellow oil, $40.4 \mathrm{mg}, 92 \%$ yield. ${ }^{1} \mathrm{H}$ NMR $\left(400 \mathrm{MHz}, \mathrm{CDCl}_{3}\right) \delta 8.39-8.32(\mathrm{~m}, 2 \mathrm{H}), 8.03-7.96(\mathrm{~m}, 2 \mathrm{H}), 7.37-7.30(\mathrm{~m}, 2 \mathrm{H})$, $7.17(\mathrm{~d}, J=8.2 \mathrm{~Hz}, 2 \mathrm{H}), 5.35(\mathrm{~d}, J=1.9 \mathrm{~Hz}, 1 \mathrm{H}), 4.27(\mathrm{dd}, J=6.4,3.4 \mathrm{~Hz}, 1 \mathrm{H}), 3.95-3.87(\mathrm{~m}, 1 \mathrm{H})$, $3.38-3.29(\mathrm{~m}, 1 \mathrm{H}), 2.00-1.91(\mathrm{~m}, 2 \mathrm{H}), 1.79-1.68(\mathrm{~m}, 1 \mathrm{H}), 1.66-1.61(\mathrm{~m}, 1 \mathrm{H}) .{ }^{13} \mathrm{C}$ NMR $(101$ $\left.\mathrm{MHz}, \mathrm{CDCl}_{3}\right) \delta 150.23,145.85,134.64,134.43,129.55,128.47,128.32,124.52,111.02,60.11$, 50.29, 42.42, 24.16, 19.82. HR-ESI-MS $\mathrm{m} / z$ calcd. for $\mathrm{C}_{18} \mathrm{H}_{16} \mathrm{ClN}_{3} \mathrm{NaO}_{4} \mathrm{~S}_{2}[\mathrm{M}+\mathrm{Na}]^{+}: 460.0163$, 
found: 460.0161 .<smiles>COc1ccc(C2[C@H](C#N)CCCN2N)cc1</smiles>

2-(4-Methoxyphenyl)-1-((4-nitrophenyl)sulfonyl)-3-thiocyanatopiperidine (3q). Flash column chromatography (eluent: $\mathrm{PE} / \mathrm{EtOAc}=15 / 1$ to $3 / 1$, v/v) to afford $3 \mathbf{q}$ as a yellow oil, $26.9 \mathrm{mg}, 62 \%$ yield. ${ }^{1} \mathrm{H}$ NMR $\left(400 \mathrm{MHz}, \mathrm{CDCl}_{3}\right) \delta 8.28(\mathrm{~d}, J=8.8 \mathrm{~Hz}, 2 \mathrm{H}), 7.91(\mathrm{~d}, J=8.8 \mathrm{~Hz}, 2 \mathrm{H}), 7.08(\mathrm{~d}, J=$ $8.7 \mathrm{~Hz}, 2 \mathrm{H}), 6.81(\mathrm{~d}, J=8.8 \mathrm{~Hz}, 2 \mathrm{H}), 5.28(\mathrm{~d}, J=2.2 \mathrm{~Hz}, 1 \mathrm{H}), 4.22(\mathrm{q}, J=3.4 \mathrm{~Hz}, 1 \mathrm{H}), 3.91-3.85$ $(\mathrm{m}, 1 \mathrm{H}), 3.78(\mathrm{~s}, 3 \mathrm{H}), 3.42-3.33(\mathrm{~m}, 1 \mathrm{H}), 2.10-2.01(\mathrm{~m}, 1 \mathrm{H}), 1.99-1.92(\mathrm{~m}, 1 \mathrm{H}), 1.87-1.77(\mathrm{~m}$, $1 \mathrm{H}), 1.69-1.62(\mathrm{~m}, 1 \mathrm{H}) .{ }^{13} \mathrm{C}$ NMR $\left(101 \mathrm{MHz}, \mathrm{CDCl}_{3}\right) \delta 159.65,150.06,146.01,128.44,128.40$, $127.60,124.32,114.59,111.27,60.43,55.51,50.59,42.53,24.36,20.16$. HR-ESI-MS $\mathrm{m} / \mathrm{z}$ calcd. for $\mathrm{C}_{19} \mathrm{H}_{19} \mathrm{~N}_{3} \mathrm{NaO}_{5} \mathrm{~S}_{2}[\mathrm{M}+\mathrm{Na}]^{+}:$456.0658, found: 456.0647 .

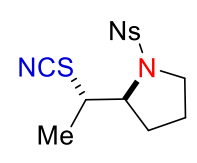

rel-(S)-1-((4-Nitrophenyl)sulfonyl)-2-((S)-1-thiocyanatoethyl)pyrrolidine (2r). Flash column chromatography (eluent: $\mathrm{PE} / \mathrm{EtOAc}=15 / 1$ to $3 / 1, \mathrm{v} / \mathrm{v}$ ) to afford $2 \mathbf{r}$ as a yellow solid, $34.0 \mathrm{mg}, 99 \%$ yield. ${ }^{1} \mathrm{H}$ NMR (400 MHz, $\left.\mathrm{CDCl}_{3}\right) \delta 8.46-8.39(\mathrm{~m}, 2 \mathrm{H}), 8.10-8.05(\mathrm{~m}, 2 \mathrm{H}), 4.12-4.03(\mathrm{~m}, 1 \mathrm{H})$, $3.93-3.87(\mathrm{~m}, 1 \mathrm{H}), 3.58-3.50(\mathrm{~m}, 1 \mathrm{H}), 3.34-3.27(\mathrm{~m}, 1 \mathrm{H}), 2.00-1.92(\mathrm{~m}, 1 \mathrm{H}), 1.90-1.81(\mathrm{~m}$, $2 \mathrm{H}), 1.57-1.50(\mathrm{~m}, 1 \mathrm{H}), 1.47(\mathrm{~d}, J=7.1 \mathrm{~Hz}, 3 \mathrm{H}) .{ }^{13} \mathrm{C}$ NMR $\left(101 \mathrm{MHz}, \mathrm{CDCl}_{3}\right) \delta 150.61,141.96$, 129.13, 124.76, 110.99, 63.50, 50.69, 46.71, 26.70, 24.66, 14.10. HR-ESI-MS m/z calcd. for $\mathrm{C}_{13} \mathrm{H}_{15} \mathrm{~N}_{3} \mathrm{NaO}_{4} \mathrm{~S}_{2}[\mathrm{M}+\mathrm{Na}]^{+}:$364.0396, found: 364.0403 .

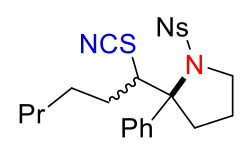

1-((4-Nitrophenyl)sulfonyl)-2-phenyl-2-(1-thiocyanatohexyl)pyrrolidine $\quad(2 \mathrm{~s})$. Flash column chromatography (eluent: $\mathrm{PE} / \mathrm{EtOAc}=20 / 1$ to $4 / 1, \mathrm{v} / \mathrm{v}$ ) to afford $2 \mathrm{~s}$ as a yellow oil, $47.3 \mathrm{mg}, 99 \%$ yield; $d r=7: 3 .{ }^{1} \mathrm{H}$ NMR (400 MHz, $\mathrm{CDCl}_{3}$ ) Major: $\delta 7.95(\mathrm{~d}, J=8.8 \mathrm{~Hz}, 1.4 \mathrm{H}), 7.31-7.16(\mathrm{~m}$, $4.2 \mathrm{H}$, overlapped with the peak of chloroform), $4.82-4.77(\mathrm{~m}, 0.7 \mathrm{H}), 3.97-3.90(\mathrm{~m}, 0.7 \mathrm{H}), 3.70-$ $3.64(\mathrm{~m}, 0.7 \mathrm{H}), 2.69-2.57(\mathrm{~m}, 0.7 \mathrm{H}), 2.48-2.37(\mathrm{~m}, 0.7 \mathrm{H}), 2.33-2.25(\mathrm{~m}, 0.7 \mathrm{H}), 2.24-2.06(\mathrm{~m}$, $1.4 \mathrm{H}), 1.99-1.65(\mathrm{~m}, 2.1 \mathrm{H}), 1.64-1.40(\mathrm{~m}, 1.4 \mathrm{H}), 1.37-1.29(\mathrm{~m}, 2.1 \mathrm{H}), 0.92-0.87(\mathrm{~m}, 2.1 \mathrm{H})$; Minor: $\delta 7.95(\mathrm{~d}, J=8.8 \mathrm{~Hz}, 0.6 \mathrm{H}), 7.31-7.16(\mathrm{~m}, 1.2 \mathrm{H}$, overlapped with the peak of chloroform), $7.09(\mathrm{~d}, J=8.8 \mathrm{~Hz}, 0.6 \mathrm{H}), 4.75-4.71(\mathrm{~m}, 0.3 \mathrm{H}), 3.84-3.77(\mathrm{~m}, 0.3 \mathrm{H}), 3.62-3.56(\mathrm{~m}, 0.3 \mathrm{H}), 2.69$ $-2.57(\mathrm{~m}, 0.6 \mathrm{H}), 2.48-2.37(\mathrm{~m}, 0.3 \mathrm{H}), 1.99-1.65(\mathrm{~m}, 1.2 \mathrm{H}), 1.64-1.40(\mathrm{~m}, 0.9 \mathrm{H}), 1.37-1.29(\mathrm{~m}$, 
0.9H), 0.99 (t, $J=7.1 \mathrm{~Hz}, 0.9 \mathrm{H}) .{ }^{13} \mathrm{C}$ NMR (101 MHz, $\left.\mathrm{CDCl}_{3}\right)$ Major: $\delta 149.34,145.55,139.65$, $128.78,128.50,127.59,127.37,123.61,111.86,74.53,58.76,51.86,36.38,31.98,31.27,27.37$, 24.58, 22.50, 14.03. HR-ESI-MS $\mathrm{m} / z$ calcd. for $\mathrm{C}_{23} \mathrm{H}_{27} \mathrm{~N}_{3} \mathrm{NaO}_{4} \mathrm{~S}_{2}[\mathrm{M}+\mathrm{Na}]^{+}:$496.1335, found: 496.1336.

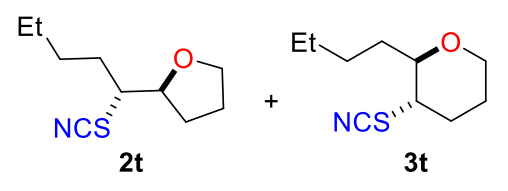

2-(1-Thiocyanatopentyl)tetrahydrofuran (2t) + 2-butyl-3-thiocyanatotetrahydro-2H-pyran (3t) Flash column chromatography (eluent: $\mathrm{PE} / \mathrm{EtOAc}=25 / 1$ to $12 / 1, \mathrm{v} / \mathrm{v}$ ) to afford the mixture of $\mathbf{2 t}$ and $\mathbf{3 t}$ as a colorless oil, $34.1 \mathrm{mg}, 85 \%$ yield (note: the polarity of $\mathbf{2 t}$ is the same to the polarity of $\mathbf{3 t}$. Their molar ratio $(\mathbf{2 t / 3 t}=100: 9)$ was determined by ${ }^{1} \mathrm{H}$ NMR. So the yield of $\mathbf{2 t}$ is equal to $\left.78 \%\right) .{ }^{1} \mathrm{H}$ NMR (400 MHz, $\left.\mathrm{CDCl}_{3}\right)$ 2t: $\delta 4.05(\mathrm{dd}, J=14.2,6.7 \mathrm{~Hz}, 1 \mathrm{H}), 3.95-3.88(\mathrm{~m}, 1 \mathrm{H}), 3.86-3.78(\mathrm{~m}$, $1 \mathrm{H}), 3.22-3.11(\mathrm{~m}, 1 \mathrm{H}), 2.17-2.09(\mathrm{~m}, 1 \mathrm{H}), 2.03-1.88(\mathrm{~m}, 3 \mathrm{H}), 1.81-1.59(\mathrm{~m}, 3 \mathrm{H}), 1.44-1.34$ $(\mathrm{m}, 3 \mathrm{H}), 0.94(\mathrm{t}, J=7.2 \mathrm{~Hz}, 3 \mathrm{H}) ; 3 \mathrm{t}: \delta 4.00-3.96(\mathrm{~m}, 0.09 \mathrm{H}), 3.48-3.41(\mathrm{~m}, 0.09 \mathrm{H}), 3.37-3.31$ $(\mathrm{m}, 0.09 \mathrm{H}), 2.91-2.80(\mathrm{~m}, 0.09 \mathrm{H}), 2.39-2.33(\mathrm{~m}, 0.09 \mathrm{H}), 2.03-1.88(\mathrm{~m}, 0.27 \mathrm{H}), 1.81-1.59(\mathrm{~m}$, $0.27 \mathrm{H}), 1.44-1.34(\mathrm{~m}, 0.27 \mathrm{H}), 0.91-0.88(\mathrm{~m}, 0.27 \mathrm{H}) .{ }^{13} \mathrm{C} \mathrm{NMR}\left(101 \mathrm{MHz}, \mathrm{CDCl}_{3}\right) 2 \mathrm{t}: \delta 111.57$, $81.13,68.97,54.77,31.46,29.20,29.17,26.10,22.37$, 13.97. HR-ESI-MS $\mathrm{m} / \mathrm{z}$ calcd. for $\mathrm{C}_{10} \mathrm{H}_{17} \mathrm{NNaOS}[\mathrm{M}+\mathrm{Na}]^{+}:$222.0923, found: 222.0926.

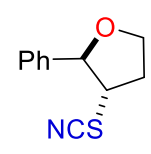

2-Phenyl-3-thiocyanatotetrahydrofuran $\mathbf{( 2 u )}$. Flash column chromatography (eluent: PE/EtOAc = $25 / 1$ to $15 / 1, \mathrm{v} / \mathrm{v})$ to afford $\mathbf{2 u}$ as a colorless oil, $19.3 \mathrm{mg}, 94 \% .{ }^{1} \mathrm{H}$ NMR $\left(400 \mathrm{MHz}, \mathrm{CDCl}_{3}\right) \delta 7.40-$ $7.32(\mathrm{~m}, 5 \mathrm{H}), 4.83(\mathrm{~d}, J=6.1 \mathrm{~Hz}, 1 \mathrm{H}), 4.31-4.25(\mathrm{~m}, 1 \mathrm{H}), 4.17-4.11(\mathrm{~m}, 1 \mathrm{H}), 3.52(\mathrm{dd}, J=13.8$, $6.0 \mathrm{~Hz}, 1 \mathrm{H}), 2.65-2.57(\mathrm{~m}, 1 \mathrm{H}), 2.32-2.24(\mathrm{~m}, 1 \mathrm{H}) .{ }^{13} \mathrm{C} \mathrm{NMR}\left(101 \mathrm{MHz}, \mathrm{CDCl}_{3}\right) \delta 138.89$, 128.91, 128.76, 125.95, 110.71, 85.74, 67.59, 52.43, 33.46. HR-ESI-MS $\mathrm{m} / \mathrm{z}$ calcd. for $\mathrm{C}_{11} \mathrm{H}_{11} \mathrm{NNaOS}[\mathrm{M}+\mathrm{Na}]^{+}:$228.0454, found: 228.0453 .

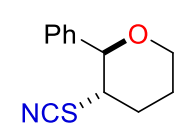

2-Phenyl-3-thiocyanatotetrahydro-2H-pyran (2v). Flash column chromatography (eluent: $\mathrm{PE} / \mathrm{EtOAc}=20 / 1$ to $5 / 1, \mathrm{v} / \mathrm{v})$ to afford $2 \mathrm{v}$ as a colorless oil, $17.9 \mathrm{mg}, 82 \% .{ }^{1} \mathrm{H}$ NMR (400 MHz, $\left.\mathrm{CDCl}_{3}\right) \delta 7.42-7.35(\mathrm{~m}, 5 \mathrm{H}), 4.29(\mathrm{~d}, J=10.0 \mathrm{~Hz}, 1 \mathrm{H}), 4.16-4.11(\mathrm{~m}, 1 \mathrm{H}), 3.65(\mathrm{td}, J=11.8,2.4$ $\mathrm{Hz}, 1 \mathrm{H}), 3.19-3.11(\mathrm{~m}, 1 \mathrm{H}), 2.53-2.47(\mathrm{~m}, 1 \mathrm{H}), 2.12-1.94(\mathrm{~m}, 2 \mathrm{H}), 1.90-1.84(\mathrm{~m}, 1 \mathrm{H}) .{ }^{13} \mathrm{C}$ 
$\operatorname{NMR}\left(101 \mathrm{MHz}, \mathrm{CDCl}_{3}\right) \delta 138.50,129.25,128.82,127.40,110.11,84.04,68.63,49.81,32.35,27.29$. HR-ESI-MS $m / z$ calcd. for $\mathrm{C}_{12} \mathrm{H}_{13} \mathrm{NNaOS}[\mathrm{M}+\mathrm{Na}]^{+}: 242.0610$, found: 242.0614 .

NCS<smiles>CCCC1(CC)OCCCO1</smiles>

2-Phenyl-2-(thiocyanatomethyl)tetrahydrofuran (2w). Flash column chromatography (eluent: $\mathrm{PE} / \mathrm{EtOAc}=25 / 1$ to $12 / 1, \mathrm{v} / \mathrm{v})$ to afford $\mathbf{2 w}$ as a colorless oil, $16.2 \mathrm{mg}, 74 \%$. ${ }^{1} \mathrm{H} \mathrm{NMR}(400 \mathrm{MHz}$, $\left.\mathrm{CDCl}_{3}\right) \delta 7.45-7.26(\mathrm{~m}, 5 \mathrm{H}), 4.11-4.04(\mathrm{~m}, 1 \mathrm{H}), 3.99-3.92(\mathrm{~m}, 1 \mathrm{H}), 3.42(\mathrm{dd}, J=32.0,13.0 \mathrm{~Hz}$, $2 \mathrm{H}), 2.42-2.27(\mathrm{~m}, 2 \mathrm{H}), 2.11-2.02(\mathrm{~m}, 1 \mathrm{H}), 1.94-1.84(\mathrm{~m}, 1 \mathrm{H}) .{ }^{13} \mathrm{C} \mathrm{NMR}\left(101 \mathrm{MHz}, \mathrm{CDCl}_{3}\right) \delta$ 143.60, 128.72, 127.90, 125.31, 113.42, 85.33, 68.80, 46.07, 37.14, 26.11. HR-ESI-MS m/z calcd. for $\mathrm{C}_{12} \mathrm{H}_{13} \mathrm{NNaOS}[\mathrm{M}+\mathrm{Na}]^{+}:$242.0610, found: 242.0607 .<smiles>O=C1CCC(CS[As])(c2ccccc2)O1</smiles>

5-Phenyl-5-(thiocyanatomethyl)dihydrofuran-2(3H)-one (2x). Flash column chromatography (eluent: PE/EtOAc $=15 / 1$ to $2 / 1$, v/v) to afford $\mathbf{2 x}$ as a white solid, $23.2 \mathrm{mg}, 99 \% .{ }^{1} \mathrm{H}$ NMR $(400$ $\left.\mathrm{MHz} \mathrm{CDCl}_{3}\right) \delta 7.55-7.32(\mathrm{~m}, 5 \mathrm{H}), 3.52(\mathrm{q}, J=14.1 \mathrm{~Hz}, 2 \mathrm{H}), 2.81-2.52(\mathrm{~m}, 4 \mathrm{H}) .{ }^{13} \mathrm{C}$ NMR $(101$ $\left.\mathrm{MHz}, \mathrm{CDCl}_{3}\right) \delta 174.80,139.83,129.28,129.24,124.94,111.90,86.63,45.29,33.06,28.58$. HR-ESI-MS $m / z$ calcd. for $\mathrm{C}_{12} \mathrm{H}_{11} \mathrm{NNaO}_{2} \mathrm{~S}[\mathrm{M}+\mathrm{Na}]^{+}: 256.0403$, found: 256.0397 .

\section{Control experiment:}

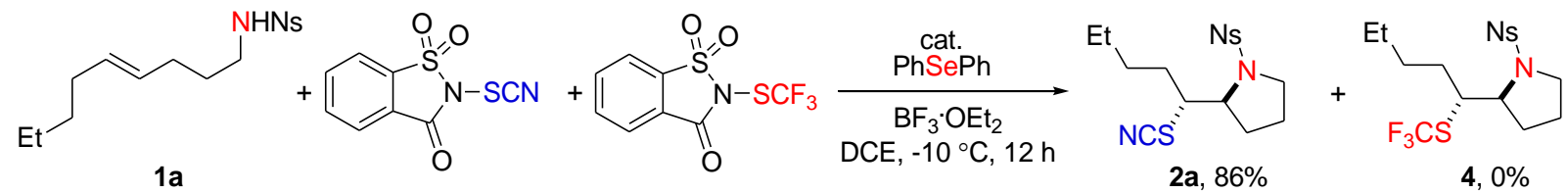

In a $10 \mathrm{~mL}$ Schlenk flask were subsequently added substrate $1 \mathrm{a}(8.2 \mathrm{mg}, 0.025 \mathrm{mmol})$, DCE $(0.5$ $\mathrm{mL}), \mathrm{PhSePh}(0.9 \mu \mathrm{L}, 20 \mathrm{~mol} \%)$, NTSc (9.0 mg, 1.5 equiv), $N$-trifluoromethylthiosaccharin (10.6 mg, 1.5 equiv), and $\mathrm{BF}_{3} \cdot \mathrm{OEt}_{2}(6.2 \mu \mathrm{L}, 2.0$ equiv $)$ in the dark. The solution was stirred in the dark at $-10{ }^{\circ} \mathrm{C}$ for $12 \mathrm{~h}$. Then it was quenched with $\mathrm{Et}_{3} \mathrm{~N}(13.9 \mu \mathrm{L}, 0.10 \mathrm{mmol})$ and concentrated in vacuo. The residue was analyzed by ${ }^{1} \mathrm{H}$ NMR using 1,3,5-trimethoxybenzene as the internal standard. NCS product $2 \mathrm{a}$ was formed in $86 \%$ yield, but $\mathrm{CF}_{3} \mathrm{~S}$ product 4 was not observed.

\section{Gram-scale experiment:}

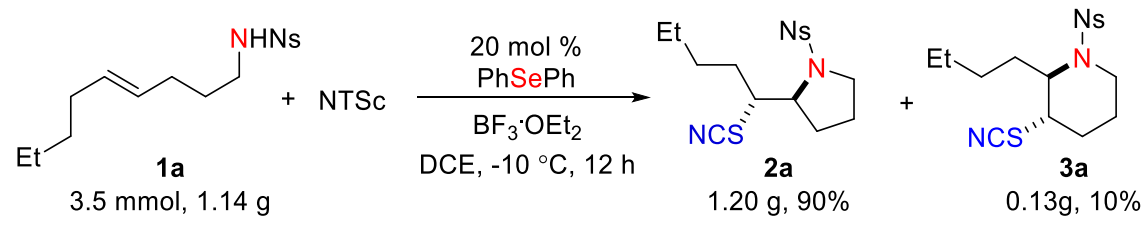


In a $200 \mathrm{~mL}$ Schlenk flask were subsequently added substrate 1a $(1.14 \mathrm{~g}, 3.5 \mathrm{mmol})$, DCE (70 mL), $\mathrm{PhSePh}(122 \mu \mathrm{L}, 20 \mathrm{~mol} \%)$, NTSc (1.26 g, 1.5 equiv), and $\mathrm{BF}_{3} \cdot \mathrm{OEt}_{2}(864 \mu \mathrm{L}, 2.0$ equiv) in the dark. The solution was stirred in the dark at $-10^{\circ} \mathrm{C}$ for $12 \mathrm{~h}$. Then it was quenched with $\mathrm{Et}_{3} \mathrm{~N}(1946 \mu \mathrm{L}$, $14.0 \mathrm{mmol}$ ) and concentrated in vacuo. The residue was purified by silica gel column chromatography to afford the corresponding saturated thiocyano-containing heterocycles $\mathbf{2 a}$ and $\mathbf{3 a}$.

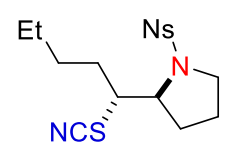

1-((4-Nitrophenyl)sulfonyl)-2-(1-thiocyanatopentyl)pyrrolidine (2a). Flash column chromatography (eluent: $\mathrm{PE} / \mathrm{EtOAc}=20 / 1$ to $7 / 1, \mathrm{v} / \mathrm{v}$ ) to afford $2 \mathbf{a}$ as a yellow solid, $1.20 \mathrm{~g}, 90 \%$ yield.<smiles>CCCCC1[C@H](C#N)CCCN1N</smiles>

2-Butyl-1-((4-nitrophenyl)sulfonyl)-3-thiocyanatopiperidine (3a). Flash column chromatography (eluent: $\mathrm{PE} / \mathrm{EtOAc}=20 / 1$ to $7 / 1, \mathrm{v} / \mathrm{v}$ ) to afford 3a as a yellow oil, $129.5 \mathrm{mg}, 10 \%$ yield. ${ }^{1} \mathrm{H}$ NMR $\left(400 \mathrm{MHz}, \mathrm{CDCl}_{3}\right) \delta 8.39-8.35(\mathrm{~m}, 2 \mathrm{H}), 8.06-8.02(\mathrm{~m}, 2 \mathrm{H}), 4.17(\mathrm{t}, J=7.4 \mathrm{~Hz}, 1 \mathrm{H}), 3.82-3.74$ (m, 2H), $3.07(\mathrm{td}, J=13.3,3.1 \mathrm{~Hz}, 1 \mathrm{H}), 2.18-2.08(\mathrm{~m}, 1 \mathrm{H}), 2.00-1.93(\mathrm{~m}, 1 \mathrm{H}), 1.80-1.60(\mathrm{~m}$, $4 \mathrm{H}), 1.34-1.25(\mathrm{~m}, 4 \mathrm{H}), 0.88(\mathrm{t}, J=7.0 \mathrm{~Hz}, 3 \mathrm{H}) .{ }^{13} \mathrm{C} \mathrm{NMR}\left(101 \mathrm{MHz}, \mathrm{CDCl}_{3}\right) \delta 150.16,146.51$, $128.41,124.52$, 111.59, 57.44, 48.88, 40.47, 30.27, 28.57, 23.57, 22.40, 19.66, 13.93. HR-ESI-MS $m / z$ calcd. for $\mathrm{C}_{16} \mathrm{H}_{21} \mathrm{~N}_{3} \mathrm{NaO}_{4} \mathrm{~S}_{2}[\mathrm{M}+\mathrm{Na}]^{+}: 406.0866$, found: 406.0876 .

\section{Further Transformations of $2 a$}

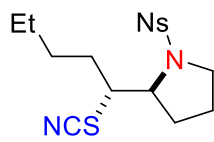

$2 \mathbf{a}$

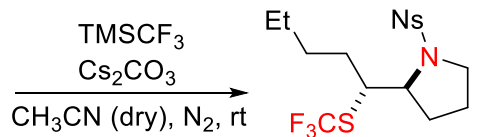

4

In a $10 \mathrm{~mL}$ Schlenk flask were subsequently added $\mathbf{2 a}(9.6 \mathrm{mg}, 0.025 \mathrm{mmol})$ and $\mathrm{Cs}_{2} \mathrm{CO}_{3}(162.9$ $\mathrm{mg}, 0.500 \mathrm{mmol})$. The flask was evacuated and flushed with nitrogen three times. Then $\mathrm{MeCN}(0.5$ $\mathrm{mL})$ and trifluoromethyltrimethylsilane $(73.9 \mu \mathrm{L}, 0.500 \mathrm{mmol})$ were added. The mixture was stirred at room temperature overnight, after which it was filtered through a short pad of Celite and concentrated in vacuo. The residue was purified by flash silica gel column chromatography to give the desired product 4 . 


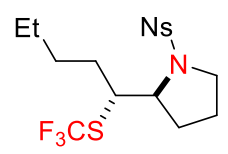

1-((4-Nitrophenyl)sulfonyl)-2-(1-((trifluoromethyl)thio)pentyl)pyrrolidine (4). Flash column chromatography (eluent: PE/EtOAc $=15 / 1$ to 4/1, v/v) to afford 4 as a colorless oil, $10.6 \mathrm{mg}, 99 \%$. ${ }^{1} \mathrm{H}$ NMR (400 MHz, $\left.\mathrm{CDCl}_{3}\right) \delta 8.47-8.33(\mathrm{~m}, 2 \mathrm{H}), 8.08-8.03(\mathrm{~m}, 2 \mathrm{H}), 3.93-3.87(\mathrm{~m}, 1 \mathrm{H}), 3.78-$ $3.73(\mathrm{~m}, 1 \mathrm{H}), 3.49-3.39(\mathrm{~m}, 2 \mathrm{H}), 1.96-1.81(\mathrm{~m}, 2 \mathrm{H}), 1.71-1.57(\mathrm{~m}, 4 \mathrm{H}), 1.46-1.33(\mathrm{~m}, 4 \mathrm{H})$, $0.95(\mathrm{t}, J=7.2 \mathrm{~Hz}, 3 \mathrm{H}) .{ }^{13} \mathrm{C} \mathrm{NMR}(101 \mathrm{MHz}, \mathrm{CDCl} 3) \delta 150.37,143.76,130.79$ (q, $\left.J=306.6 \mathrm{~Hz}\right)$, 128.83, 124.57, 63.21, 52.14, 50.06, 33.76, 29.23, 28.46, 24.94, 22.41, 14.01. ${ }^{19} \mathrm{~F}$ NMR (376 MHz, $\mathrm{CDCl} 3) \delta$-38.60. HR-ESI-MS $\mathrm{m} / z$ calcd. for $\mathrm{C}_{16} \mathrm{H}_{21} \mathrm{~F}_{3} \mathrm{~N}_{2} \mathrm{NaO}_{4} \mathrm{~S}_{2}[\mathrm{M}+\mathrm{Na}]^{+}$: 449.0787, found: 449.0787 .

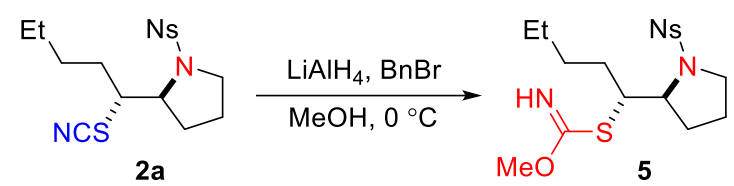

To a solution of $\mathbf{2 a}(38.3 \mathrm{mg}, 0.10 \mathrm{mmol})$ in $\mathrm{MeOH}(2.0 \mathrm{~mL})$ were subsequently added $\mathrm{LiAlH}_{4}$ (11.4 $\mathrm{mg}, 0.30 \mathrm{mmol})$ and $\mathrm{BnBr}(29.7 \mu \mathrm{L}, 0.25 \mathrm{mmol})$ at $0{ }^{\circ} \mathrm{C}$. The resulting mixture was stirred at 0 ${ }^{\circ} \mathrm{C}$ for $12 \mathrm{~h}$. Then another portion of $\mathrm{LiAlH}_{4}(11.4 \mathrm{mg}, 0.30 \mathrm{mmol})$ and $\mathrm{BnBr}(29.7 \mu \mathrm{L}, 0.25 \mathrm{mmol})$ were added. After $12 \mathrm{~h}$, the same operation was conducted. The resulting mixture was stirred for additional $12 \mathrm{~h}$, after which it was quenched with a little amount of ice and filtered through a short pad of Celite. The solution was concentrated in vacuo. The residue was purified by silica gel column chromatography to afford carbonimidothioate $\mathbf{5}$.

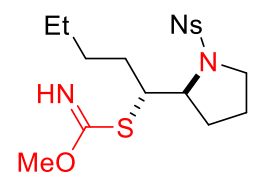

$O$-Methyl-(1-(1-((4-nitrophenyl)sulfonyl)pyrrolidin-2-yl)pentyl)carbonimidothioate (5). Flash column chromatography (eluent: $\mathrm{PE} / \mathrm{EtOAc}=15 / 1$ to 3/1, v/v) to afford 5 as a white solid, $36.6 \mathrm{mg}$, 88\%. ${ }^{1} \mathrm{H}$ NMR $\left(400 \mathrm{MHz}, \mathrm{CDCl}_{3}\right) \delta 8.38(\mathrm{~d}, J=8.7 \mathrm{~Hz}, 2 \mathrm{H}), 8.04(\mathrm{~d}, J=8.7 \mathrm{~Hz}, 2 \mathrm{H}), 7.62(\mathrm{br}, \mathrm{s}$, $1 \mathrm{H}), 3.88(\mathrm{~s}, 4 \mathrm{H}), 3.83-3.77(\mathrm{~m}, 1 \mathrm{H}), 3.44-3.38(\mathrm{~m}, 2 \mathrm{H}), 1.96-1.88(\mathrm{~m}, 1 \mathrm{H}), 1.77$ (dd, $J=14.1$, $7.1 \mathrm{~Hz}, 2 \mathrm{H}), 1.66-1.56(\mathrm{~m}, 2 \mathrm{H}), 1.49-1.27(\mathrm{~m}, 5 \mathrm{H}), 0.94(\mathrm{t}, J=7.1 \mathrm{~Hz}, 3 \mathrm{H}) .{ }^{13} \mathrm{C} \mathrm{NMR}(101 \mathrm{MHz}$, $\left.\mathrm{CDCl}_{3}\right) \delta 165.43,150.31,143.75,128.80,124.53,63.80,55.57,52.72,50.25,33.09,29.55,28.23$, 25.05, 22.52, 14.05. HR-ESI-MS $m / z$ calcd. for $\mathrm{C}_{17} \mathrm{H}_{26} \mathrm{~N}_{3} \mathrm{O}_{5} \mathrm{~S}_{2}[\mathrm{M}+\mathrm{H}]^{+} 416.1308$, found: 416.1310. 


\section{General Procedure for Catalytic Asymmetric Synthesis of Saturated Thiocyano-Containing Heterocycles}

Catalytic asymmetric thiocyanoaminocyclization:<smiles>CCC/C=C/CCCNS(=O)(=O)[O-]</smiles>

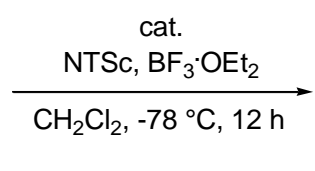<smiles></smiles><smiles>CCCNC1c2ccccc2CC1[Se]c1c(C)cccc1OC</smiles>

In a $10 \mathrm{~mL}$ Schlenk flask were subsequently added $1 \mathbf{a}(8.2 \mathrm{mg}, 0.025 \mathrm{mmol})$, DCM (0.5 mL), chiral catalyst C9 $\left(2.1 \mathrm{mg}, 20 \mathrm{~mol} \%\right.$ ), NTSc (9.0 mg, 1.5 equiv), and $\mathrm{BF}_{3} \cdot \mathrm{OEt}_{2}(6.2 \mu \mathrm{L}, 2.0$ equiv) in the dark. The solution was stirred in the dark at $-78{ }^{\circ} \mathrm{C}$ for $12 \mathrm{~h}$. Then it was quenched with $\mathrm{Et}_{3} \mathrm{~N}$ $(13.9 \mu \mathrm{L}, 0.10 \mathrm{mmol})$ and concentrated in vacuo. The residue was analyzed by ${ }^{1} \mathrm{H}$ NMR using 1,3,5-trimethoxybenzene as the internal standard. Product 2a was formed in 85\% NMR yield with $16 \%$ ee. HPLC (Daicel Chiralpak IA column, $i-\mathrm{PrOH} / \mathrm{h}$ exane $=2 / 98,1 \mathrm{~mL} / \mathrm{min}, 267 \mathrm{~nm}$ ) $\mathrm{t}_{1}=48.9$ $\min$ (minor), $\mathrm{t}_{2}=63.3 \mathrm{~min}$ (major).

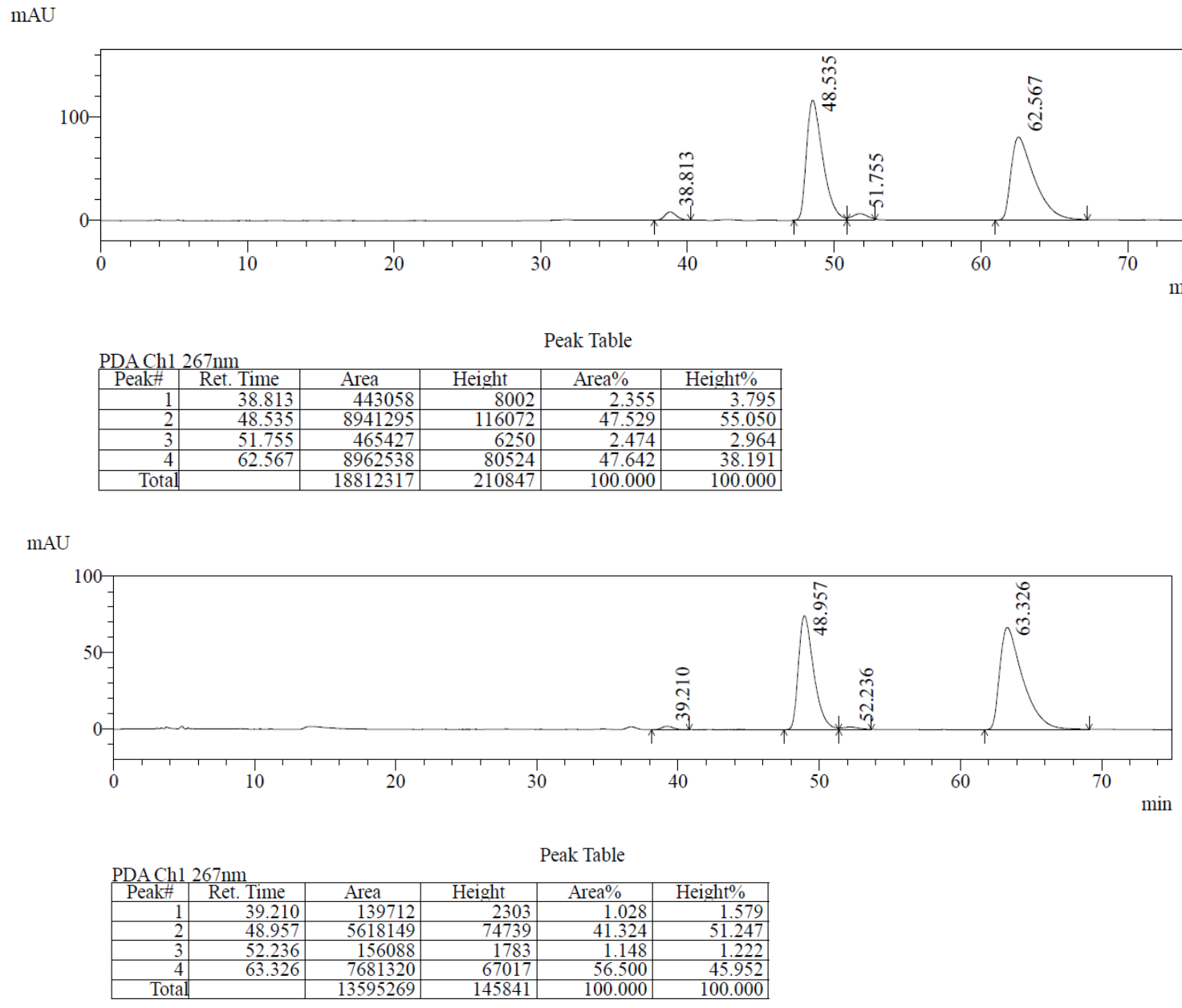


Catalytic asymmetric thiocyanooxycyclization:

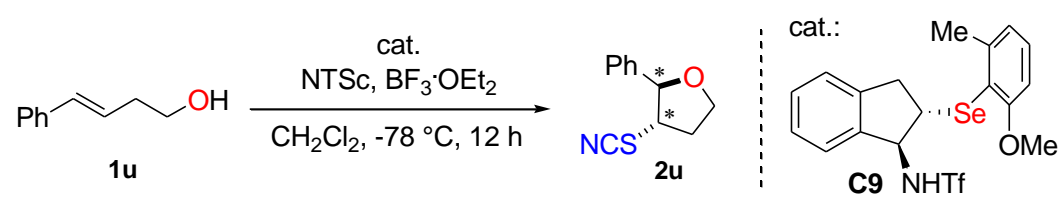

In a $10 \mathrm{~mL}$ Schlenk flask were subsequently added $1 \mathbf{u}(5.9 \mathrm{mg}, 0.04 \mathrm{mmol})$, DCM $(0.8 \mathrm{~mL})$, chiral catalyst $\mathbf{C 9}$ (3.7 mg, $20 \mathrm{~mol} \%$ ), NTSc (14.4 mg, 1.5 equiv), and $\mathrm{BF}_{3} \cdot \mathrm{OEt}_{2}(9.9 \mu \mathrm{L}, 2.0$ equiv) in the dark. The solution was stirred in the dark at $-78{ }^{\circ} \mathrm{C}$ for $12 \mathrm{~h}$. Then it was quenched with $\mathrm{Et}_{3} \mathrm{~N}$ (22 $\mu \mathrm{L}, 0.16 \mathrm{mmol}$ ) and concentrated in vacuo. The residue was analyzed by ${ }^{1} \mathrm{H}$ NMR using benzyl benzoate as the internal standard. Product $\mathbf{2 u}$ was formed in $88 \%$ NMR yield with $46 \%$ ee. HPLC (Daicel Chiralpak ID column, $i-\mathrm{PrOH} /$ hexane $=5 / 95,1 \mathrm{~mL} / \mathrm{min}, 227 \mathrm{~nm}$ ) $\mathrm{t}_{1}=9.6 \mathrm{~min}$ (major), $\mathrm{t}_{2}=$ $11.4 \min$ (minor).

$\mathrm{mAU}$
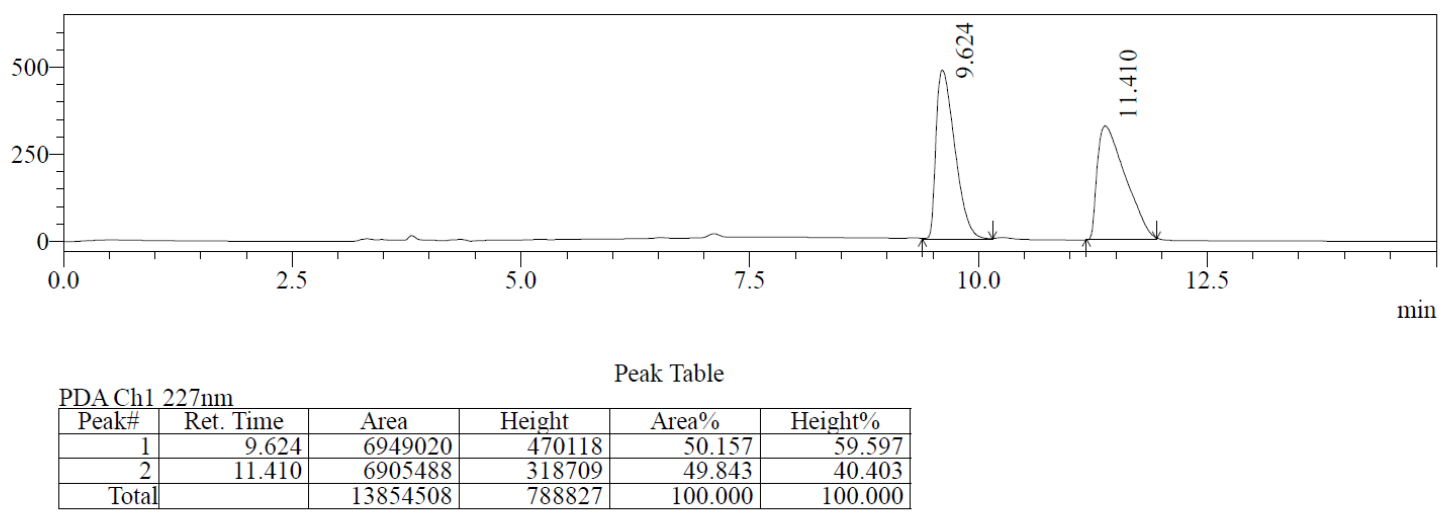

$\mathrm{mAU}$

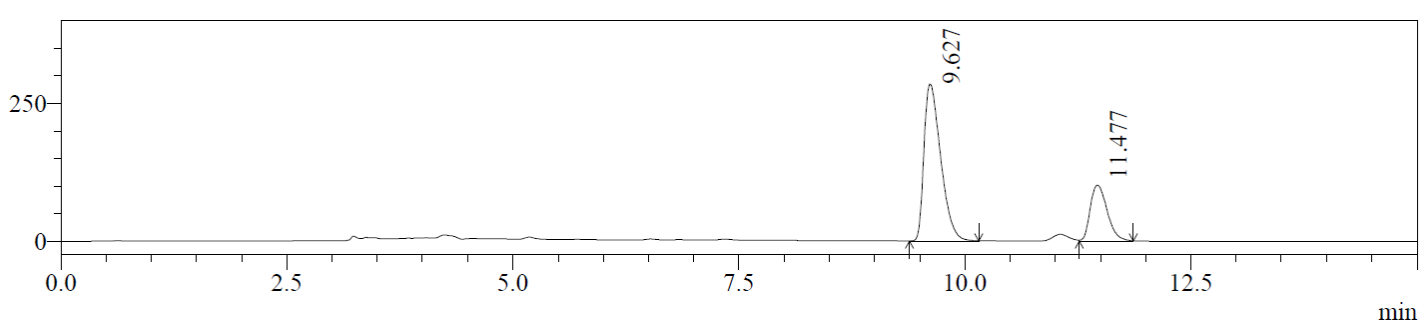

Peak Table

PDA Ch1 $227 \mathrm{~nm}$
\begin{tabular}{r|r|r|r|r|r|}
\hline Peak\# & Ret. Time & Area & \multicolumn{1}{|c|}{ Height } & \multicolumn{1}{|c|}{ Area $\%$} & Height $\%$ \\
\hline 1 & 9.627 & 3667311 & 269115 & 73.391 & 73.541 \\
\hline 2 & 11.477 & 1329607 & 96826 & 26.609 & 26.459 \\
\hline Total & & 4996918 & 365941 & 100.000 & 100.000 \\
\hline
\end{tabular}




\section{NMR Spectra for New Compounds}

${ }^{1} \mathrm{H}$ NMR (400 MHz, $\mathrm{CDCl}_{3}$ ) spectrum of (E)-4-Nitro- $N$-(non-4-en-1-yl)benzenesulfonamide (1a)

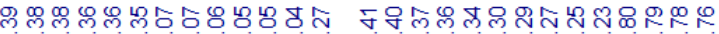

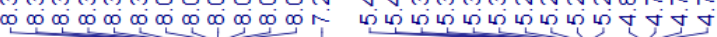

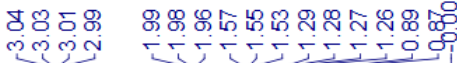
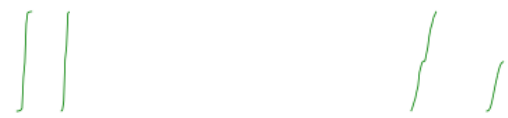

Me $\sim \sim_{\mathrm{NHNS}}$

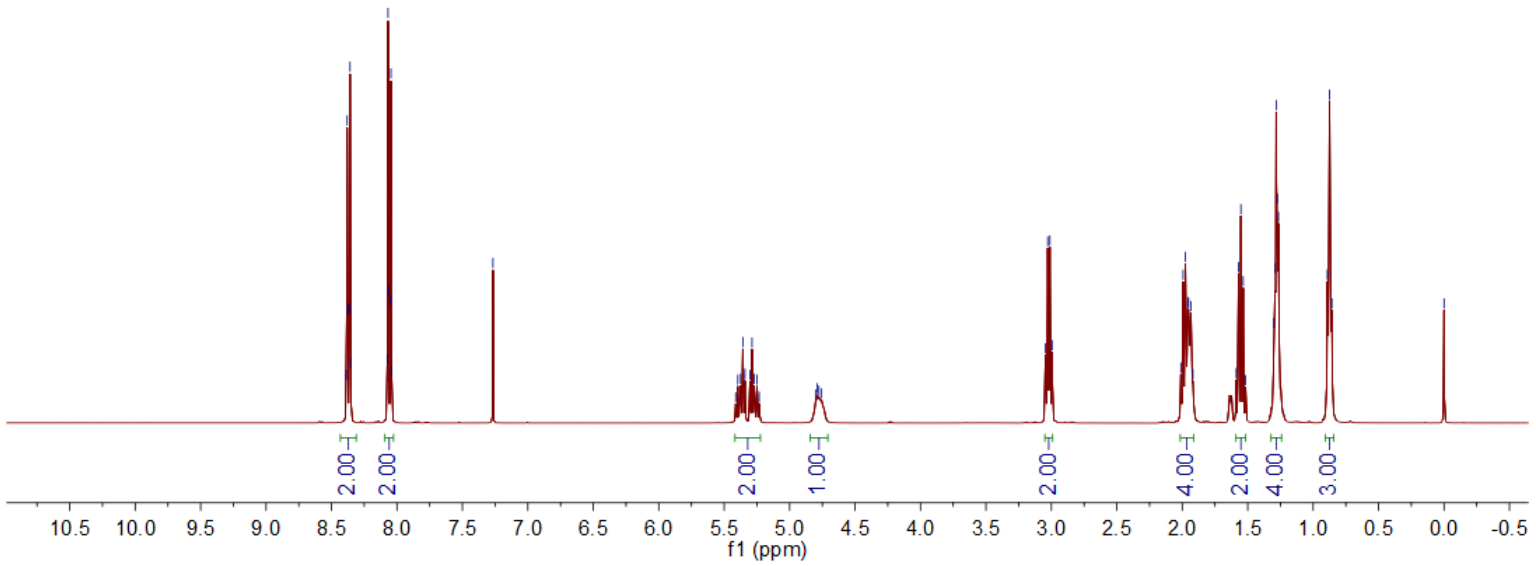

${ }^{13} \mathrm{C}$ NMR (101 MHz, $\mathrm{CDCl}_{3}$ ) spectrum of (E)-4-Nitro- $\mathrm{N}$-(non-4-en-1-yl)benzenesulfonamide (1a)

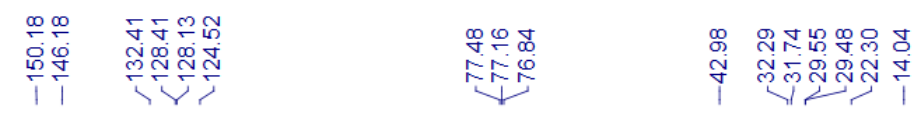

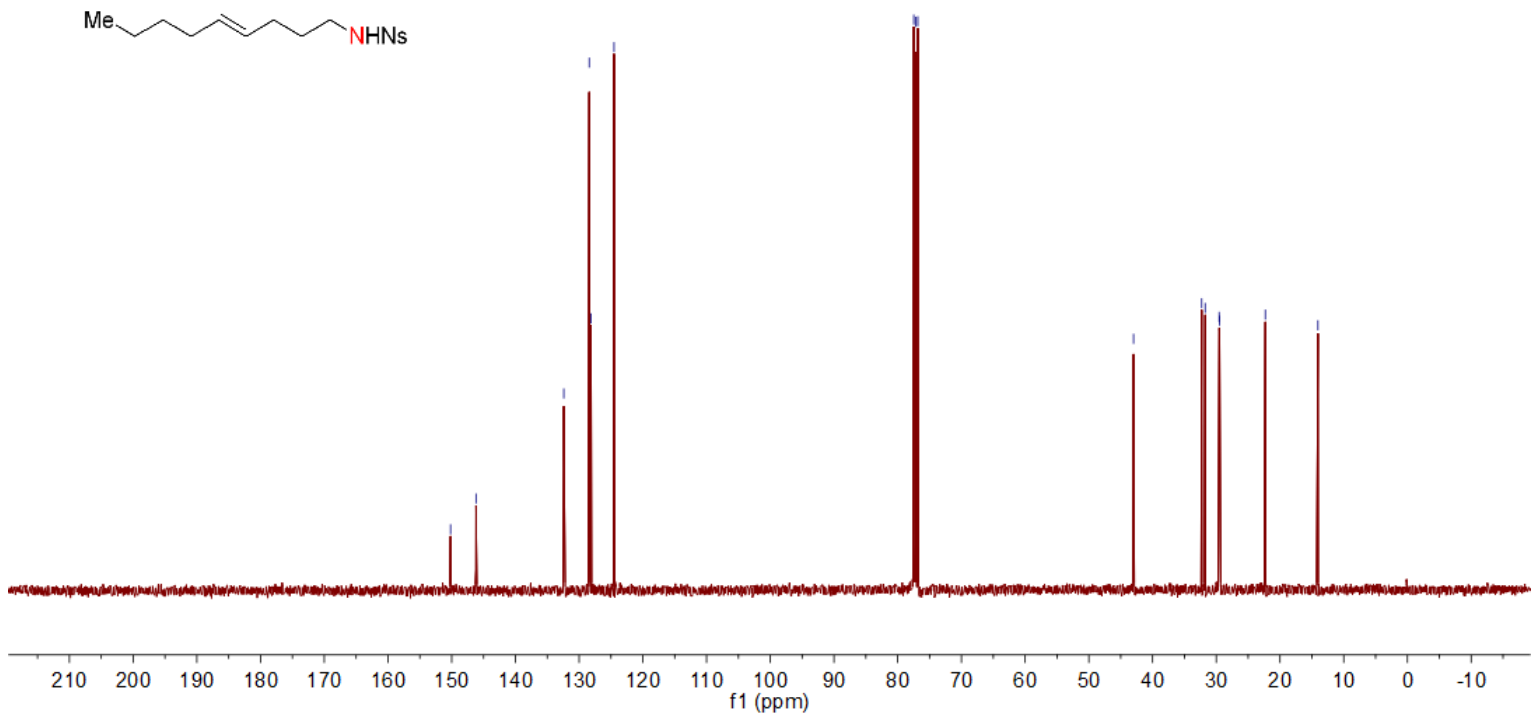


${ }^{1} \mathrm{H}$ NMR (400 MHz, $\mathrm{CDCl}_{3}$ ) spectrum of $\boldsymbol{N}$-(Hept-4-en-1-yl)-4-nitrobenzenesulfonamide (1b)

প్ల ळ

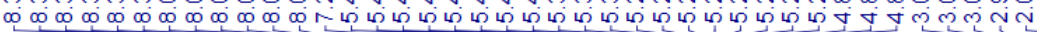
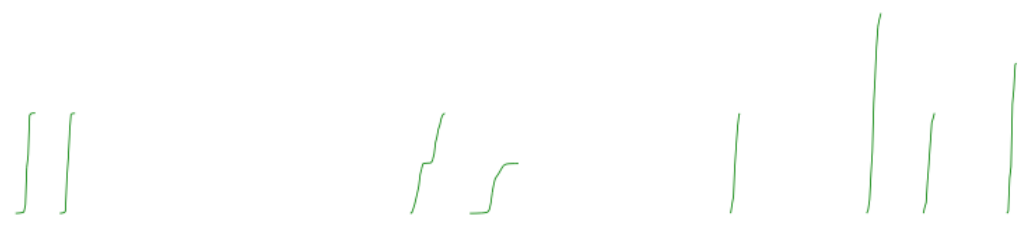

$\mathrm{Me}_{\mathrm{NHNS}}$

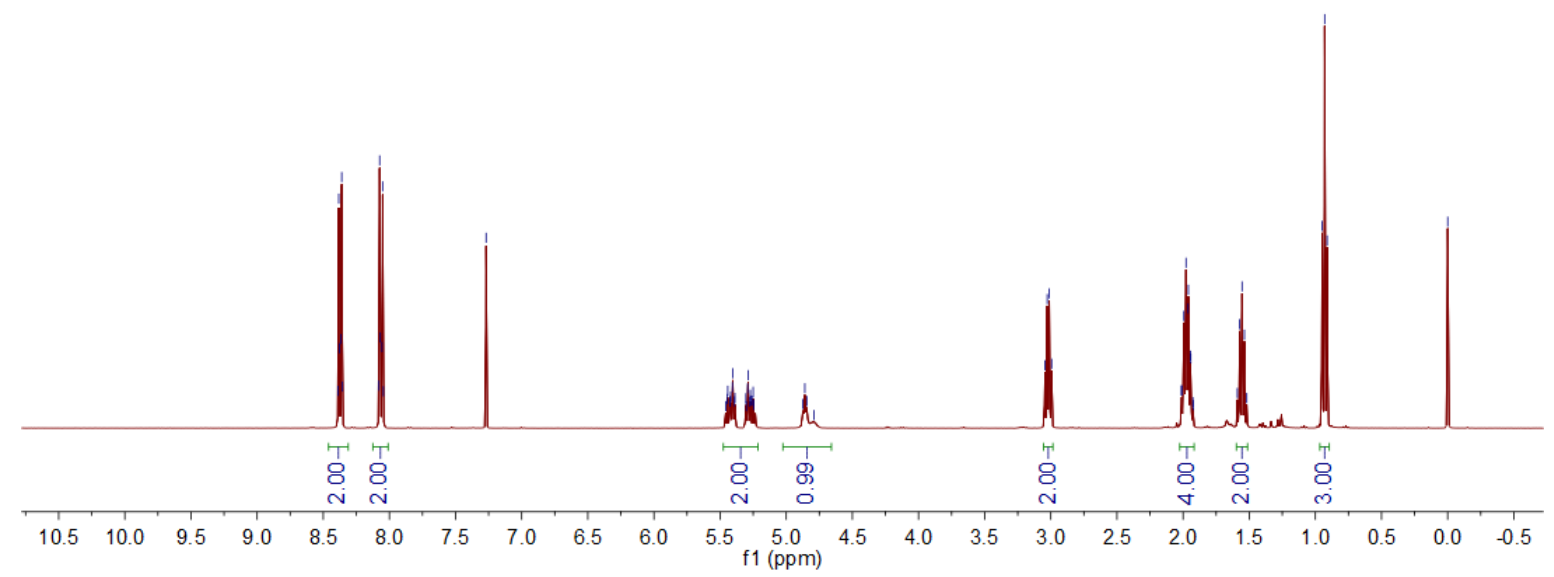

${ }^{13} \mathrm{C}$ NMR (101 MHz, $\mathrm{CDCl}_{3}$ ) spectrum of $\mathrm{N}$-(Hept-4-en-1-yl)-4-nitrobenzenesulfonamide (1b)

\begin{tabular}{|c|c|c|c|}
\hline 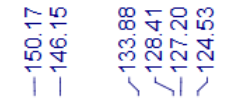 & 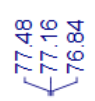 & 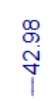 & 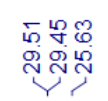 \\
\hline
\end{tabular}

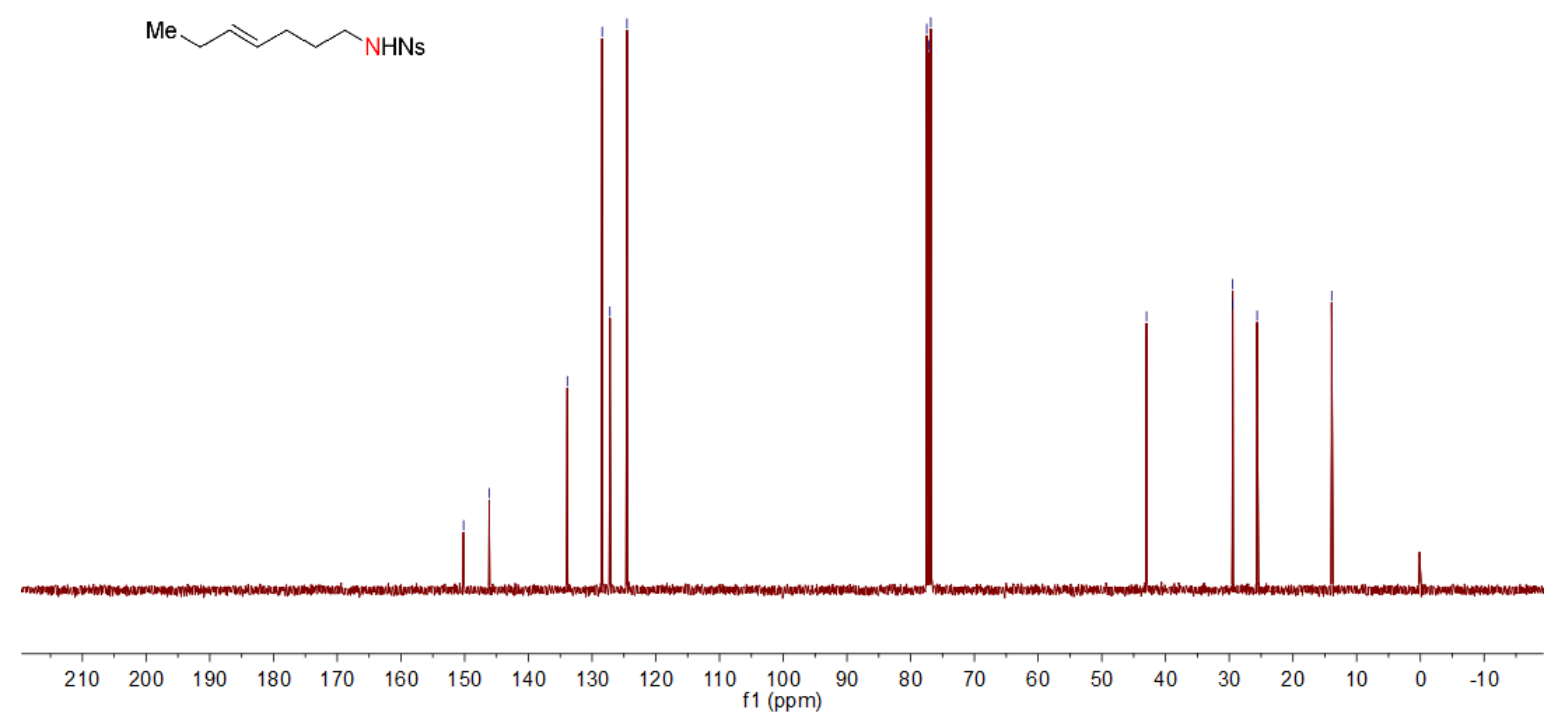


${ }^{1} \mathrm{H}$ NMR (400 MHz, $\mathrm{CDCl}_{3}$ ) spectrum of $\boldsymbol{N}$-(9-Chloronon-4-en-1-yl)-4-nitrobenzenesulfonamide (1d)

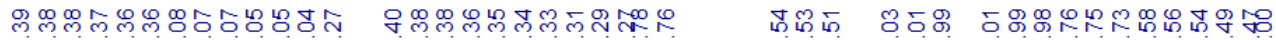

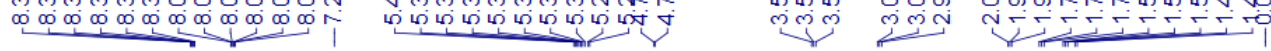
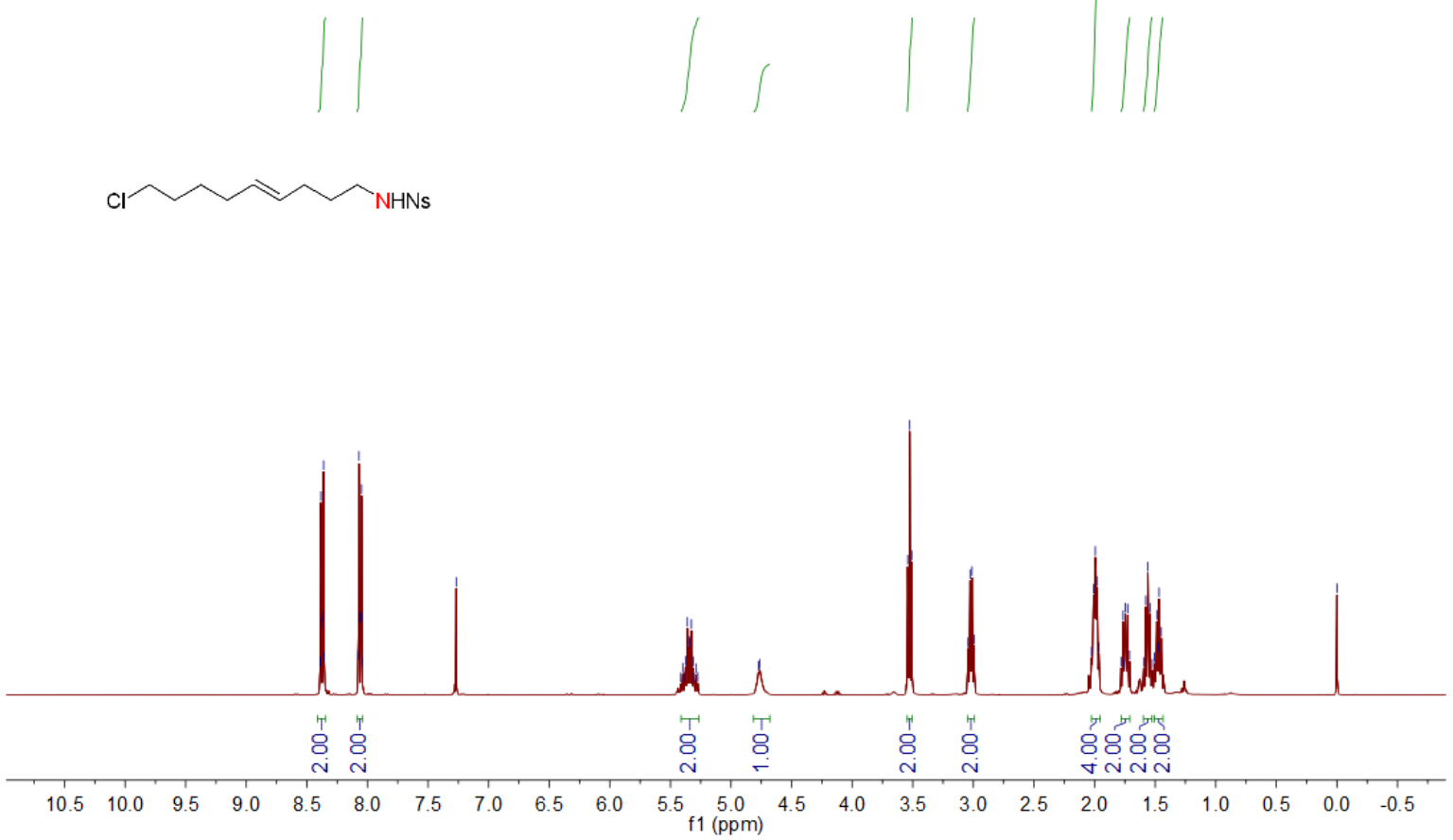

${ }^{13} \mathrm{C}$ NMR (101 MHz, $\mathrm{CDCl}_{3}$ ) spectrum of $\mathbf{N}$-(9-Chloronon-4-en-1-yl)-4-nitrobenzenesulfonamide (1d)

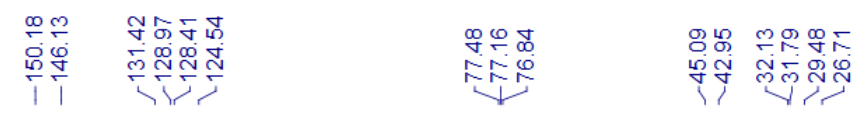

$\overbrace{\mathrm{NHNS}}$

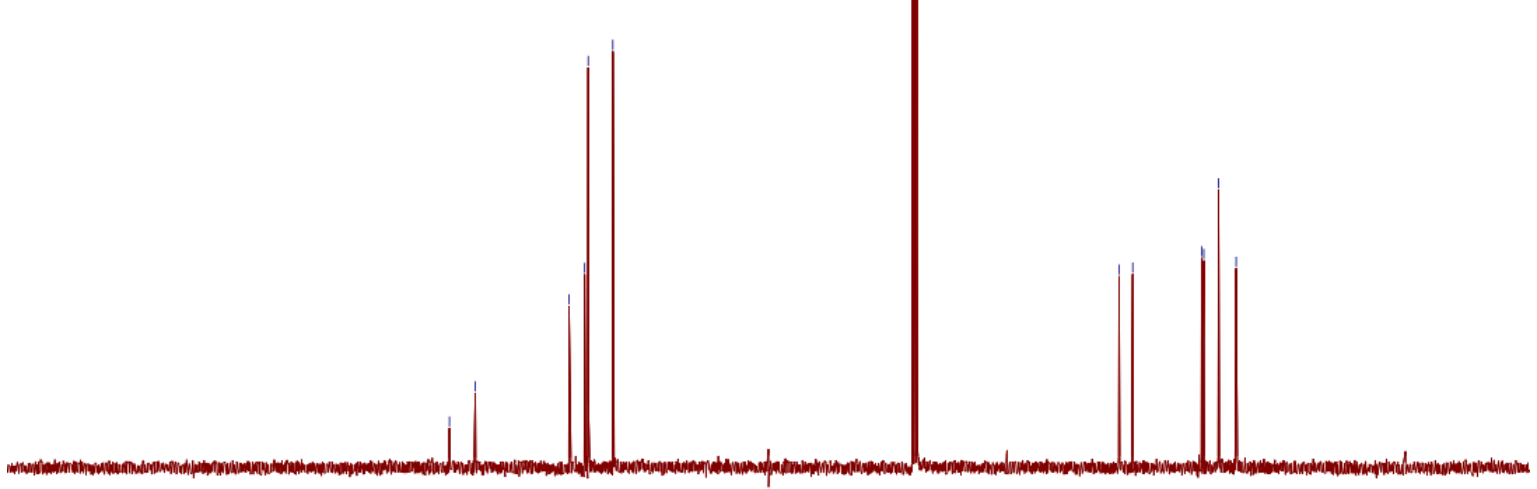

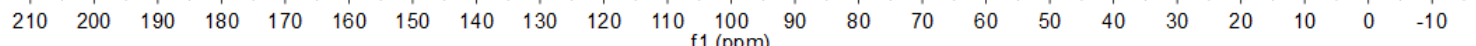


${ }^{1} \mathrm{H}$ NMR (400 MHz, $\mathrm{CDCl}_{3}$ ) spectrum of $\boldsymbol{N}$-(7-Methyloct-4-en-1-yl)-4-nitrobenzenesulfonamide (1e)

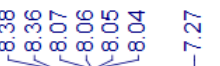
మా మ โ๐

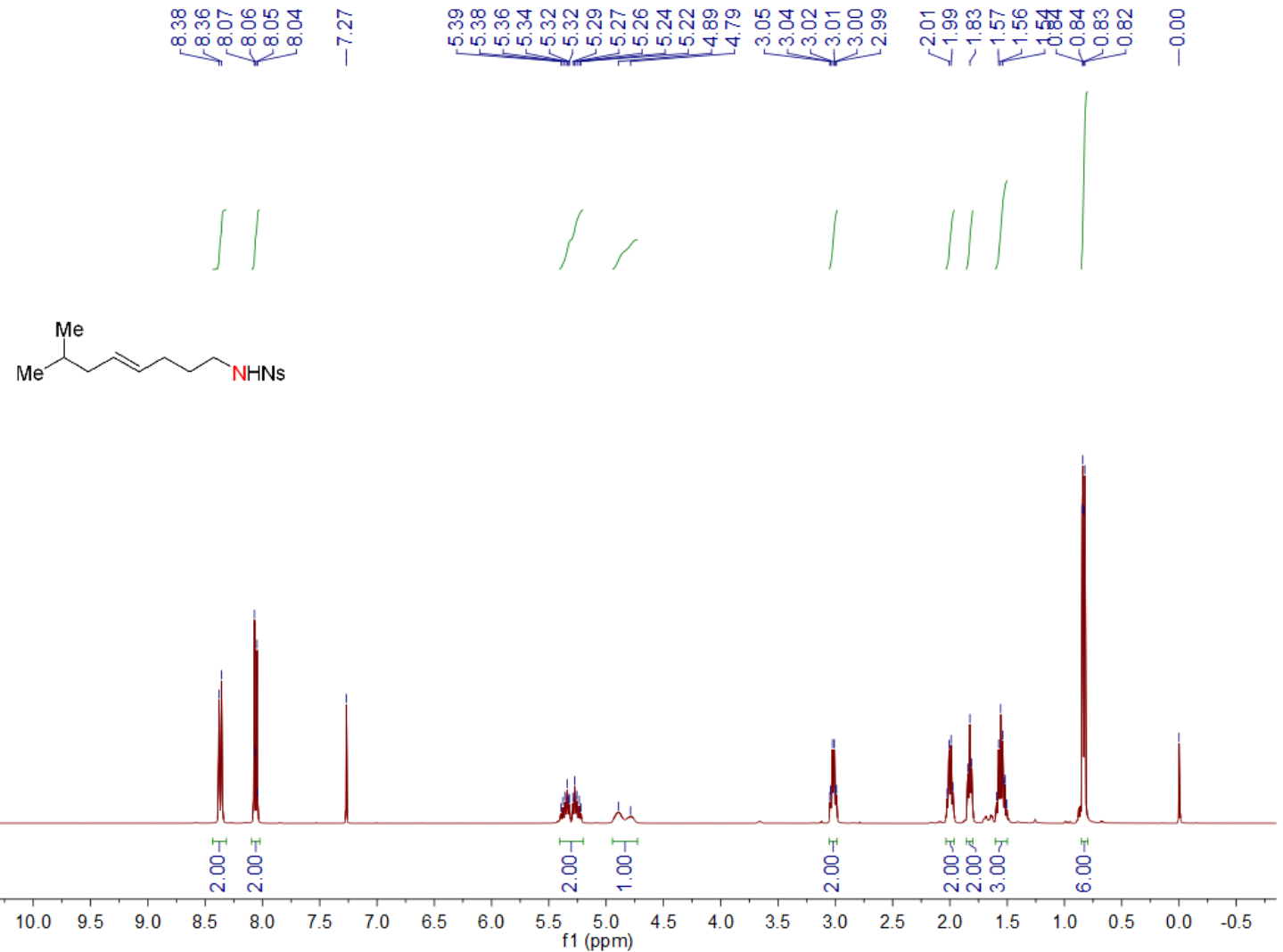

${ }^{13} \mathrm{C}$ NMR (101 MHz, $\mathrm{CDCl}_{3}$ ) spectrum of $\mathrm{N}$-(7-Methyloct-4-en-1-yl)-4-nitrobenzenesulfonamide (1e)

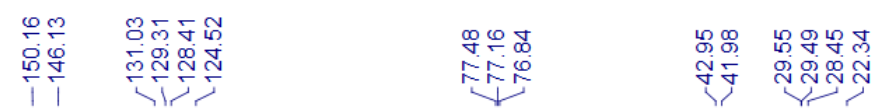

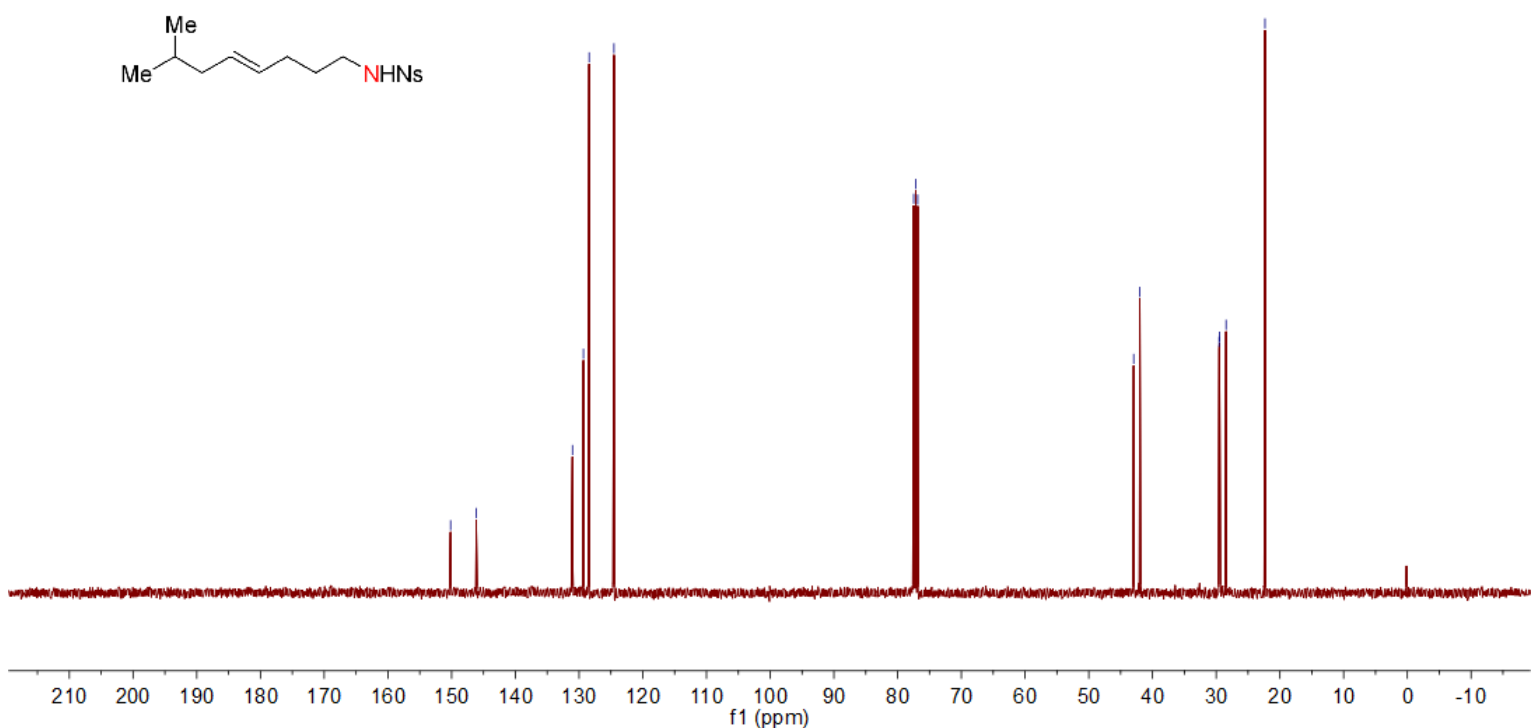


${ }^{1} \mathrm{H}$ NMR (400 MHz, $\mathrm{CDCl}_{3}$ ) spectrum of $\boldsymbol{N}$-(6-Ethyldec-4-en-1-yl)-4-nitrobenzenesulfonamide (1f)

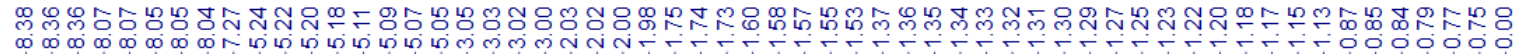
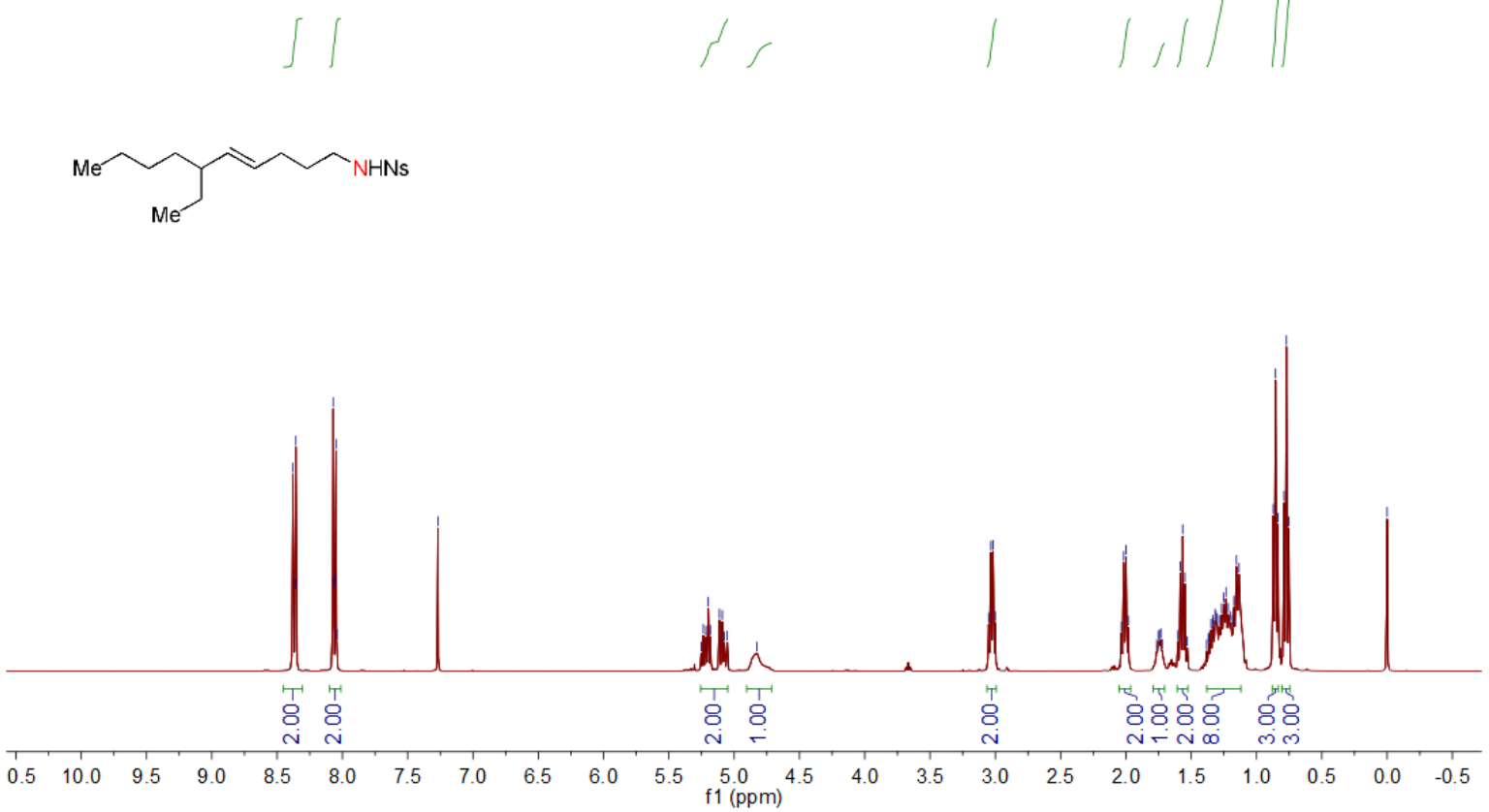

${ }^{13} \mathrm{C}$ NMR (101 MHz, $\mathrm{CDCl}_{3}$ ) spectrum of $\boldsymbol{N}$-(6-Ethyldec-4-en-1-yl)-4-nitrobenzenesulfonamide (1f)

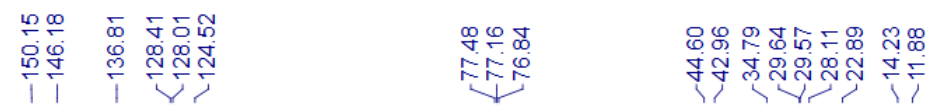

$\overbrace{M e}^{M_{N}} \overbrace{\mathrm{NHN}}$

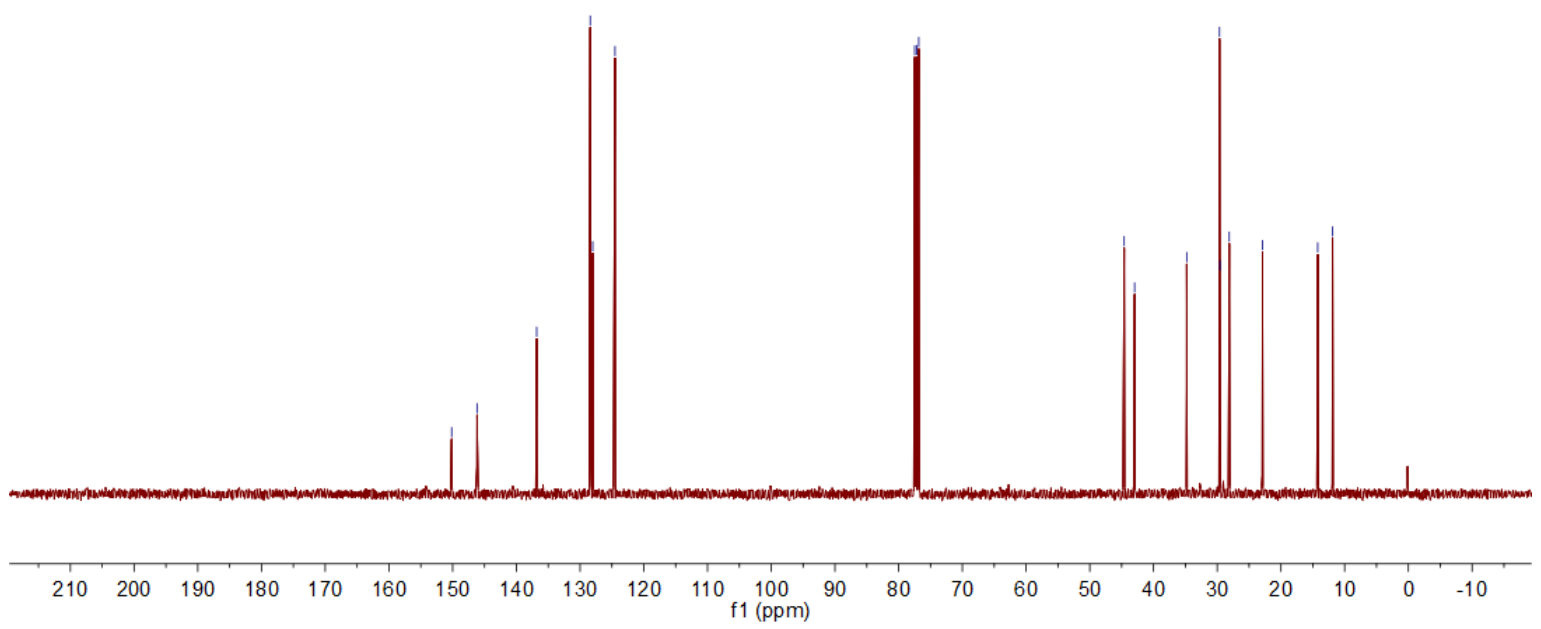


${ }^{1} \mathrm{H}$ NMR (400 MHz, $\mathrm{CDCl}_{3}$ ) spectrum of $\boldsymbol{N}$-(5-Cyclohexylpent-4-en-1-yl)-4-nitrobenzenesulfonamide (1g)

б 舟

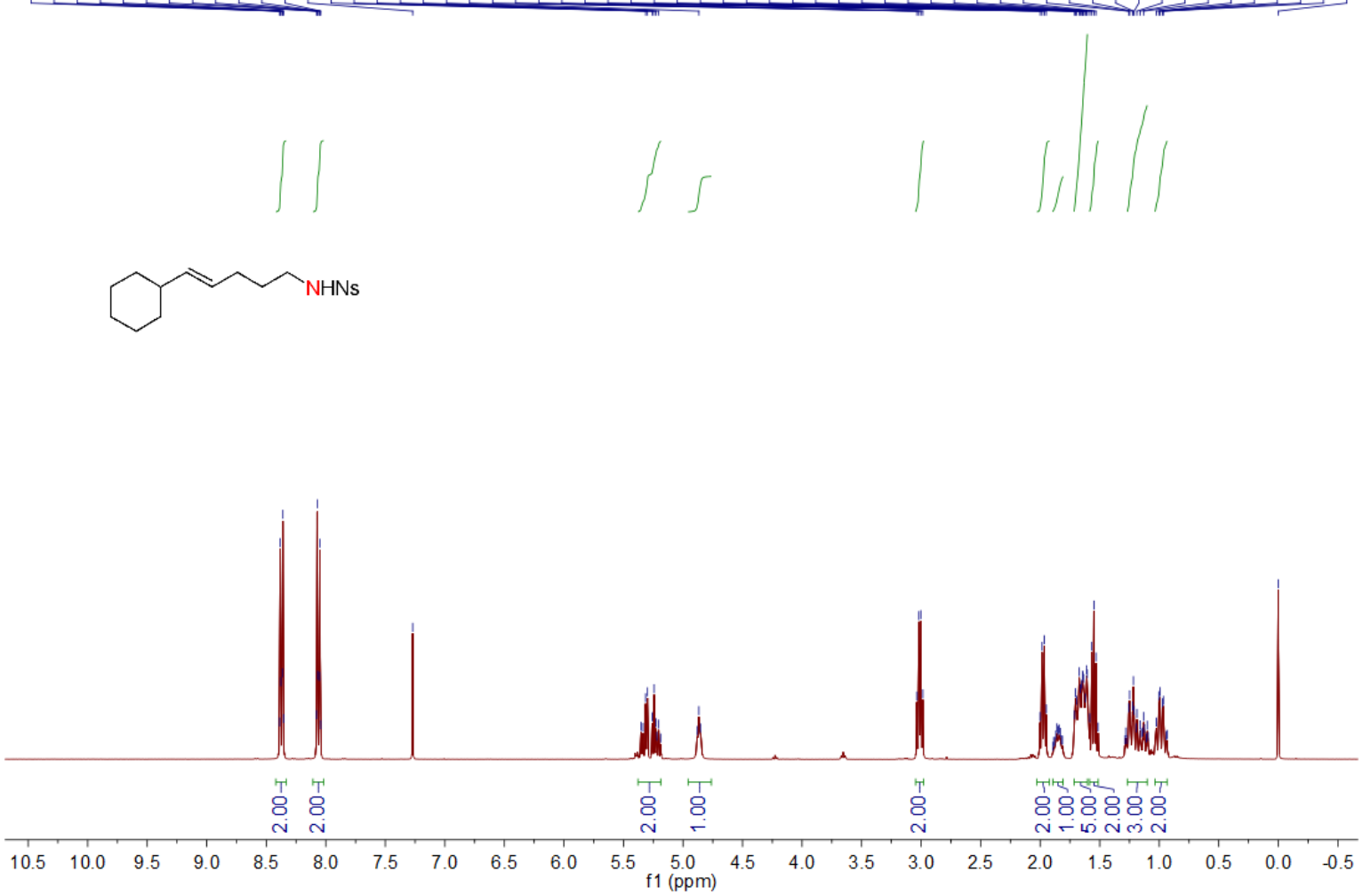

${ }^{13} \mathrm{C}$ NMR (101 MHz, $\left.\mathrm{CDCl}_{3}\right)$ spectrum of $\mathrm{N}$-(5-Cyclohexylpent-4-en-1-yl)-4-nitrobenzenesulfonamide (1g)

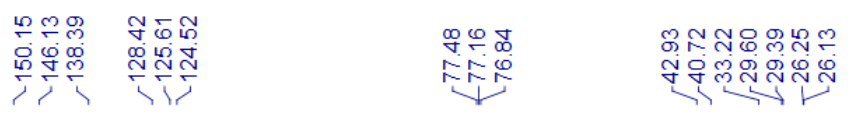

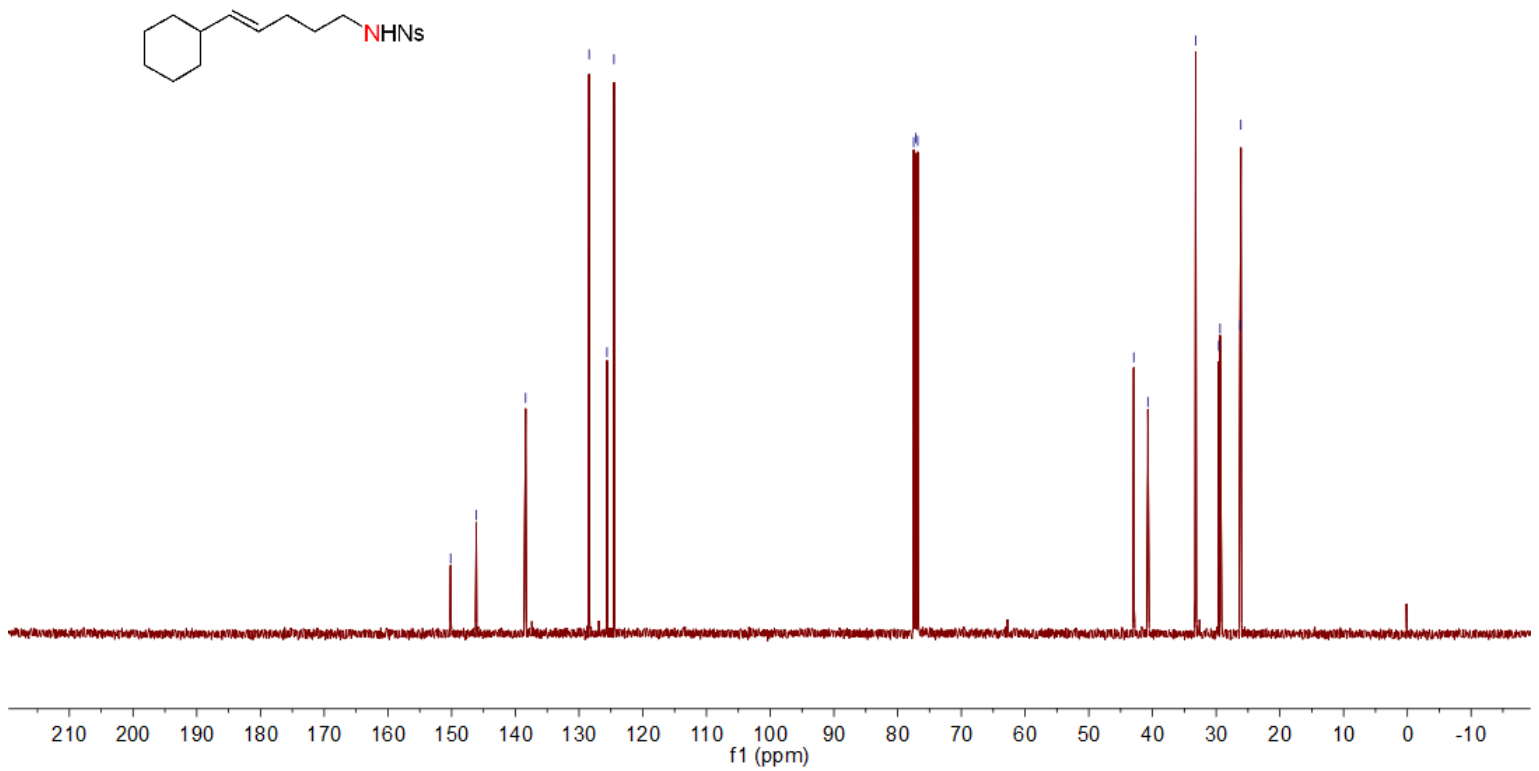


${ }^{1} \mathrm{H}$ NMR (400 MHz, $\mathrm{CDCl}_{3}$ ) spectrum of $\mathbf{N}$-(6,6-Dimethylhept-4-en-1-yl)-4-nitrobenzenesulfonamide (1h)

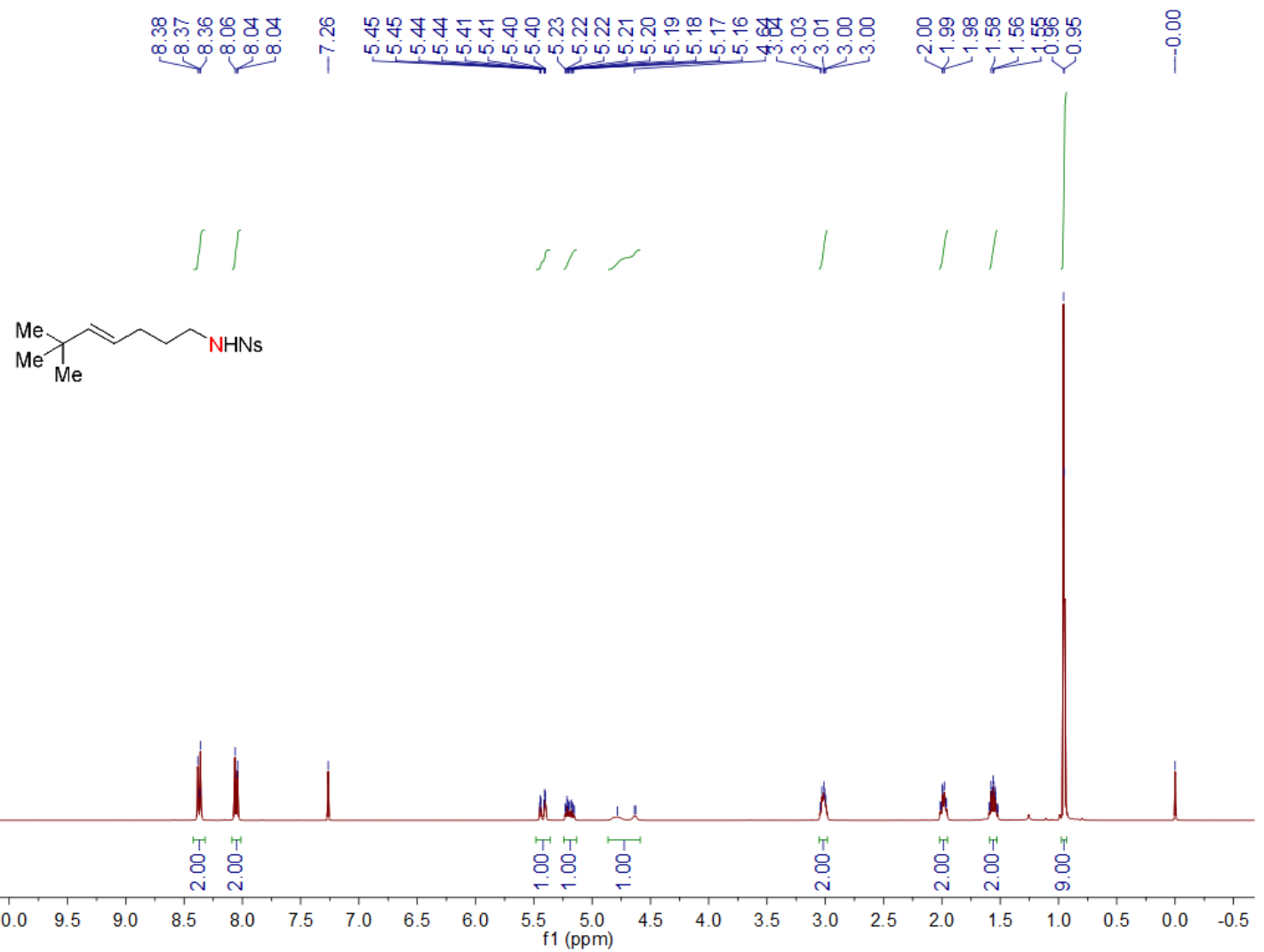

${ }^{13} \mathrm{C}$ NMR (101 MHz, $\mathrm{CDCl}_{3}$ ) spectrum of $\mathbf{N}$-(6,6-Dimethylhept-4-en-1-yl)-4-nitrobenzenesulfonamide (1h)

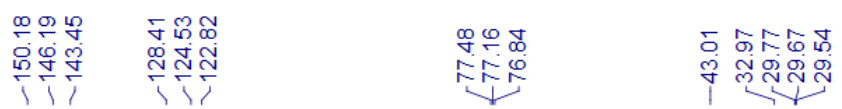

$\mathrm{Me}_{\mathrm{MHNS}}^{\mathrm{Me}}$

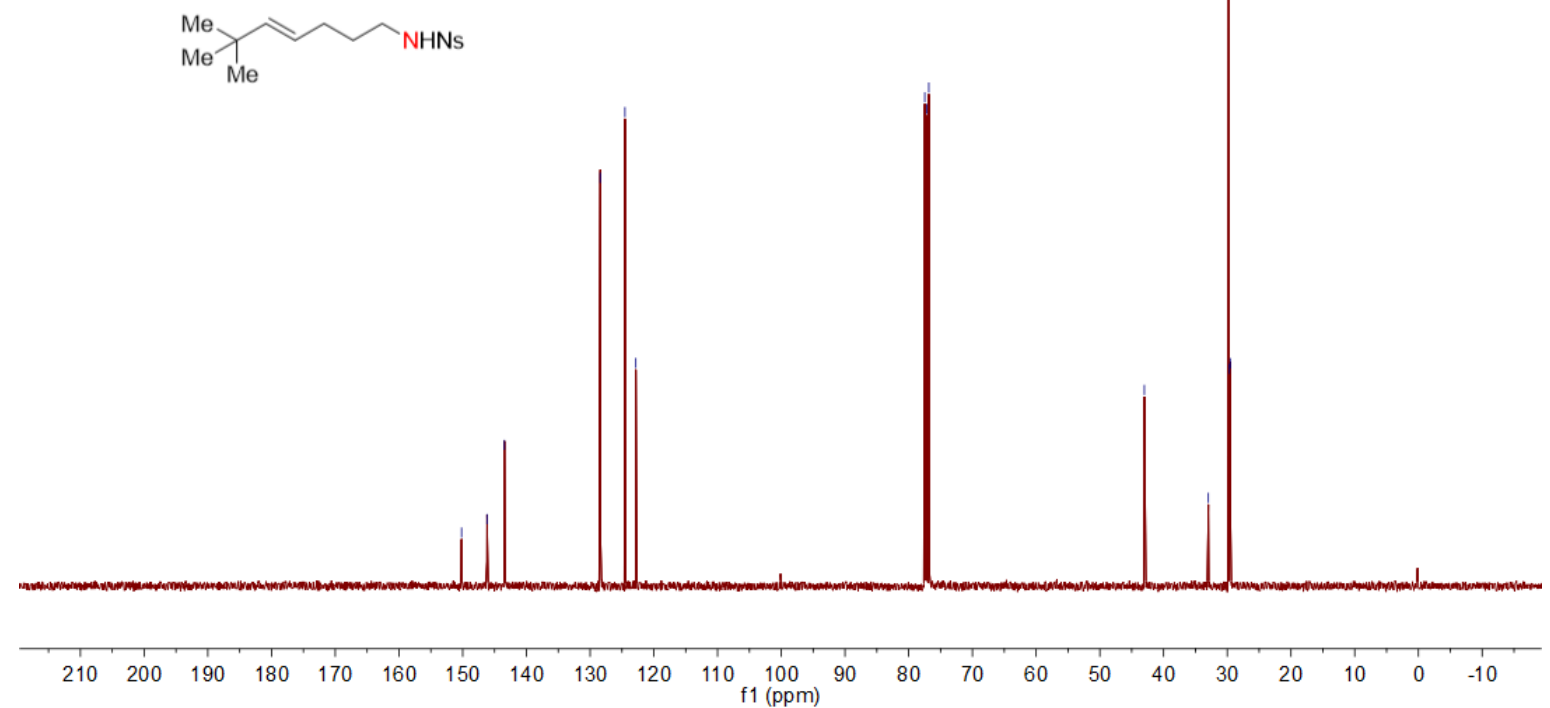


${ }^{1} \mathrm{H}$ NMR (400 MHz, $\mathrm{CDCl}_{3}$ ) spectrum of $\mathbf{N}$-(2,2-Dimethylhept-4-en-1-yl)-4-nitrobenzenesulfonamide (1k) প్ల

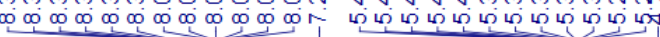
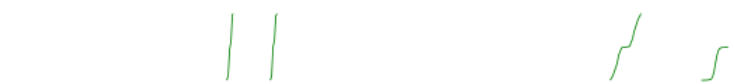

$\overbrace{M e}^{\mathrm{MHN}}$
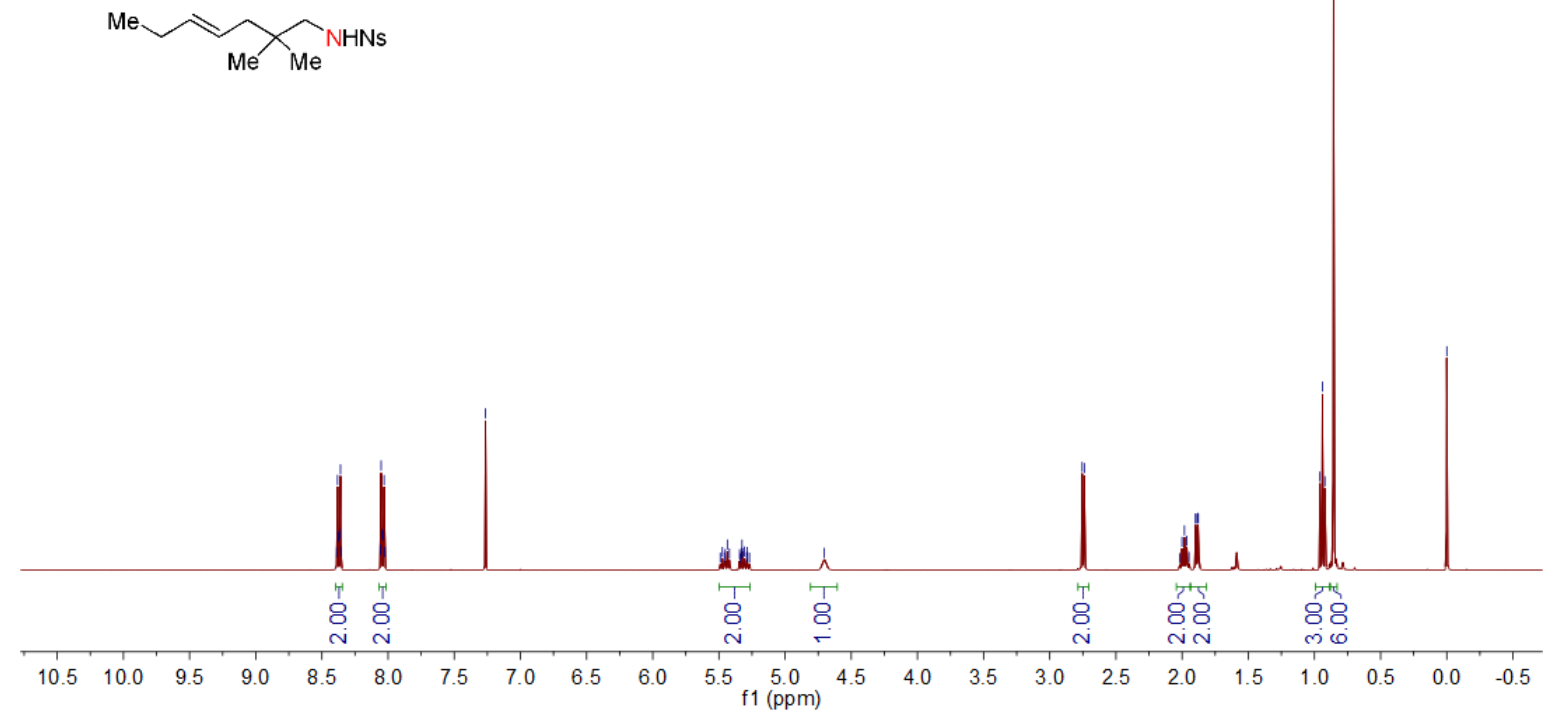

${ }^{13} \mathrm{C}$ NMR (101 MHz, $\mathrm{CDCl}_{3}$ ) spectrum of $N$-(2,2-Dimethylhept-4-en-1-yl)-4-nitrobenzenesulfonamide (1k)

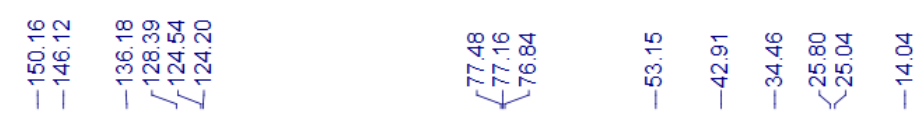

$\overbrace{M e}^{\mathrm{NHNS}}$

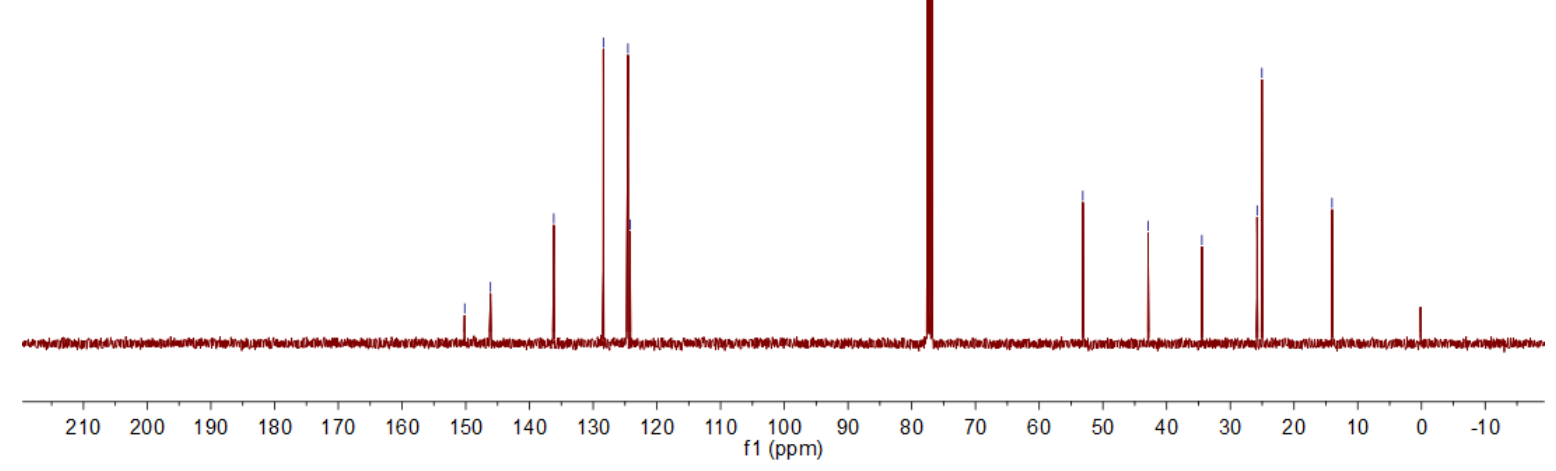


${ }^{1} \mathrm{H}$ NMR (400 MHz, $\mathrm{CDCl}_{3}$ ) spectrum of 4-Methyl- $\mathrm{N}$-(non-4-en-1-yl)benzenesulfonamide (11)

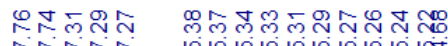

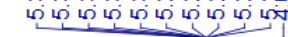

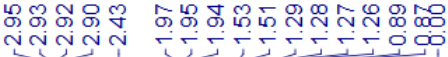
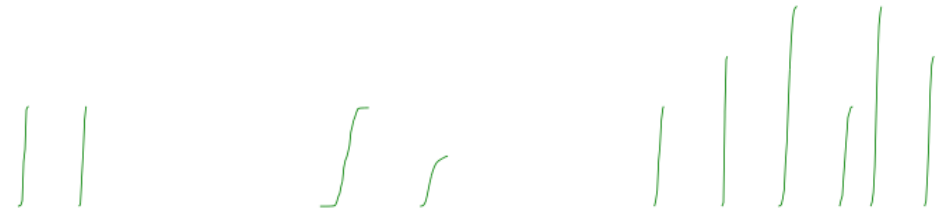

$\sim_{\mathrm{NHTS}}^{\mathrm{Me}}$

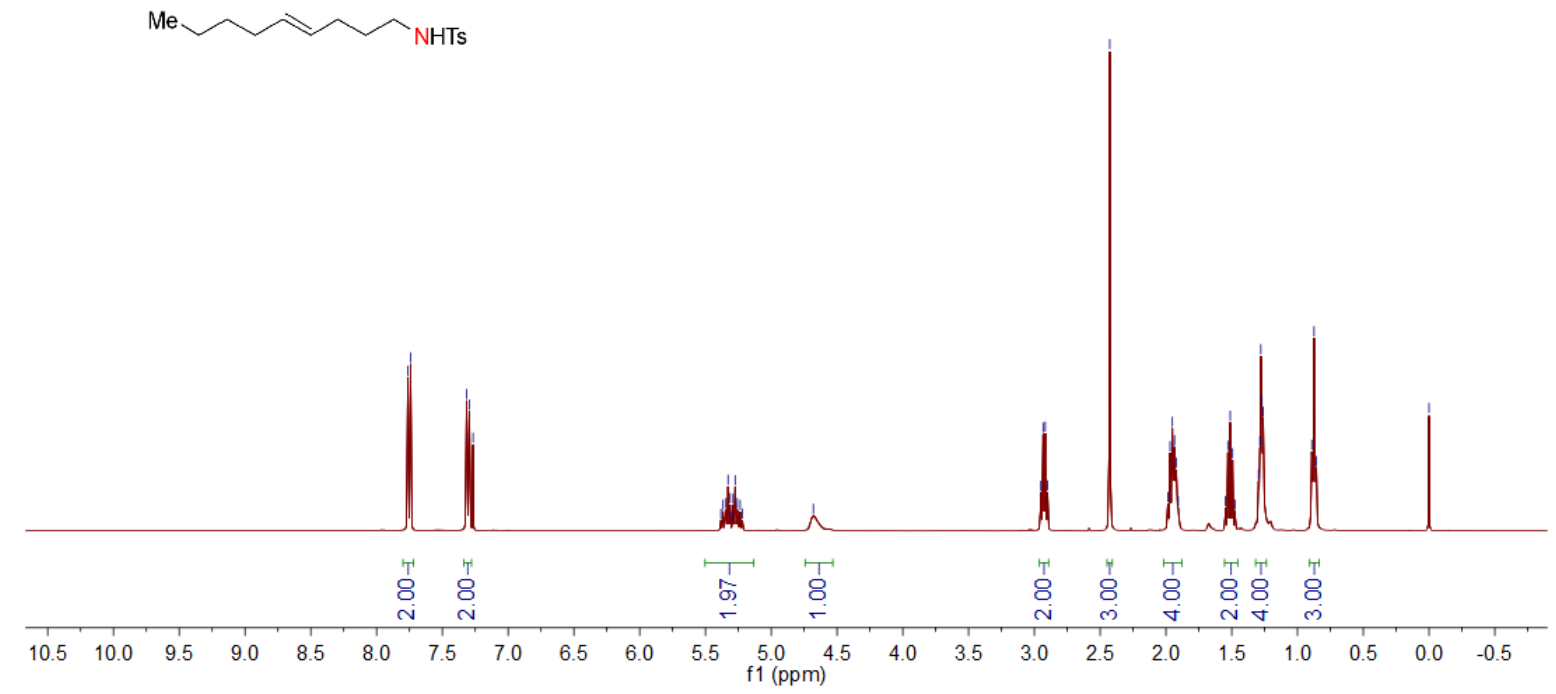

${ }^{13} \mathrm{C}$ NMR (101 MHz, $\mathrm{CDCl}_{3}$ ) spectrum of 4-Methyl- $N$-(non-4-en-1-yl)benzenesulfonamide (11)

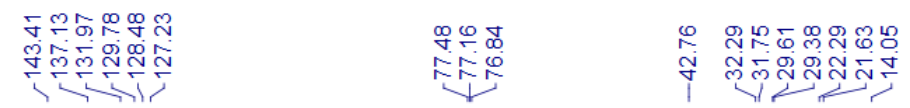

$\mathrm{Me}_{\mathrm{NHTS}}$

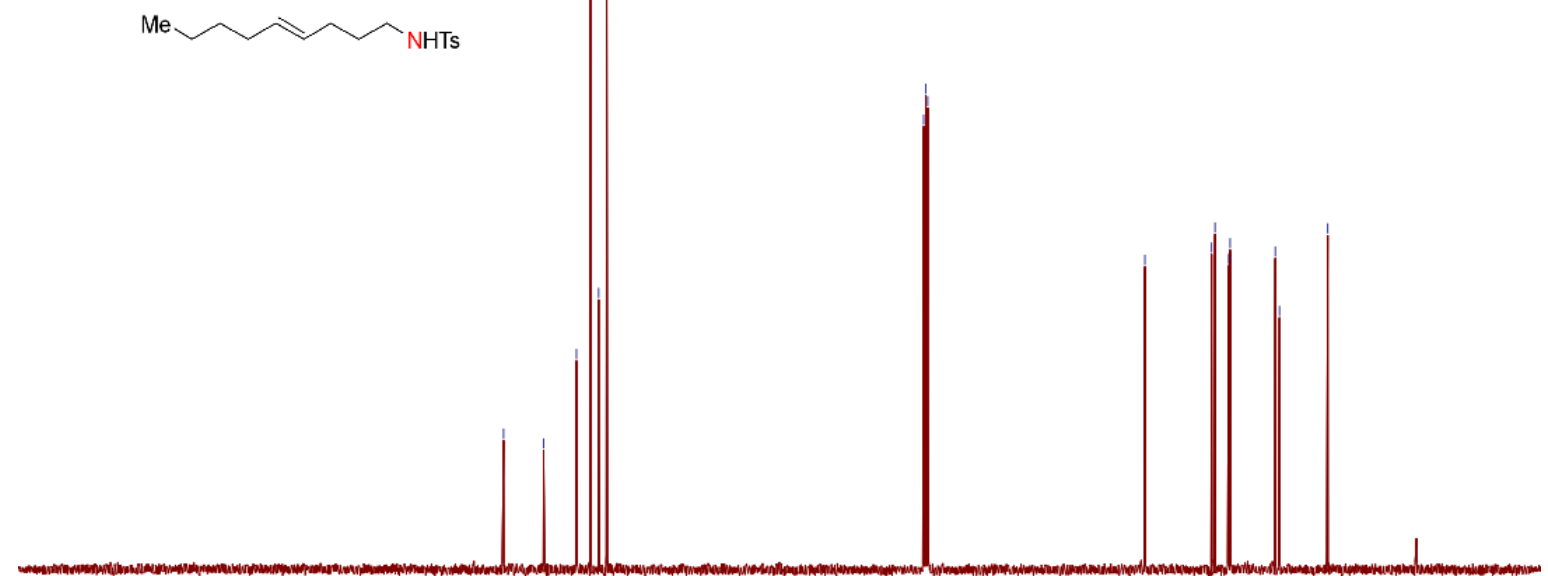

$\begin{array}{lllllllllllllllllllllll}210 & 200 & 190 & 180 & 170 & 160 & 150 & 140 & 130 & 120 & 110 & 100 & 90 & 80 & 70 & 60 & 50 & 40 & 30 & 20 & 10 & 0 & -10\end{array}$ 
${ }^{1} \mathrm{H}$ NMR (400 MHz, $\mathrm{CDCl}_{3}$ ) spectrum of $\mathrm{N}$-(Non-4-en-1-yl)methanesulfonamide (1m)

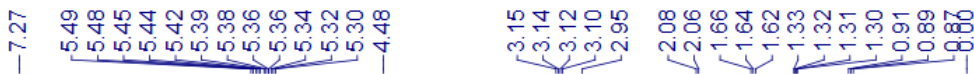
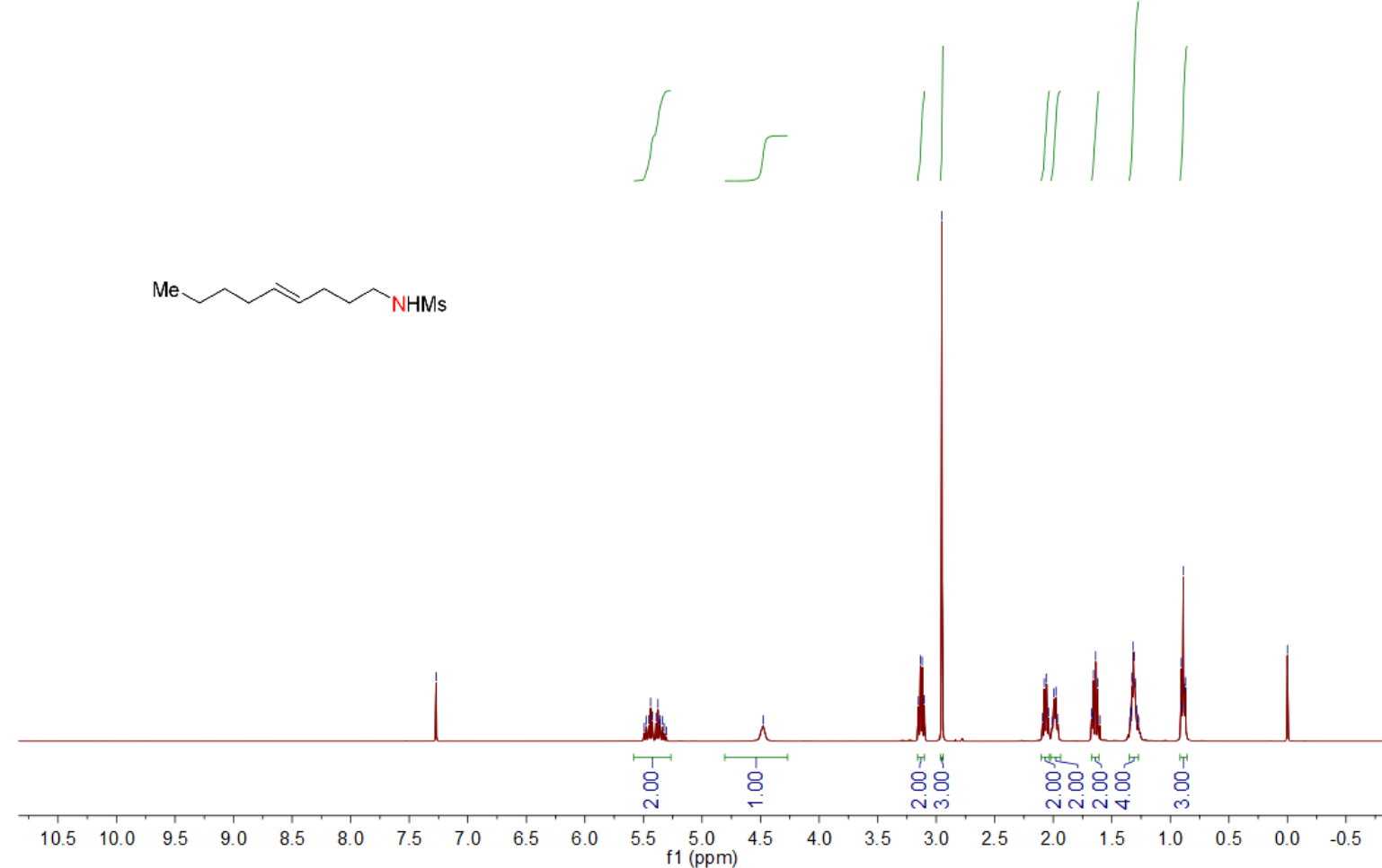

${ }^{13} \mathrm{C}$ NMR (101 MHz, $\mathrm{CDCl}_{3}$ ) spectrum of $\mathrm{N}$-(Non-4-en-1-yl)methanesulfonamide (1m)

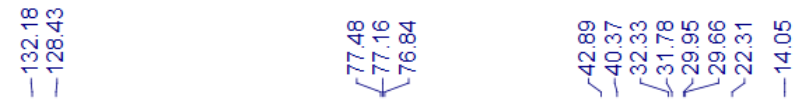

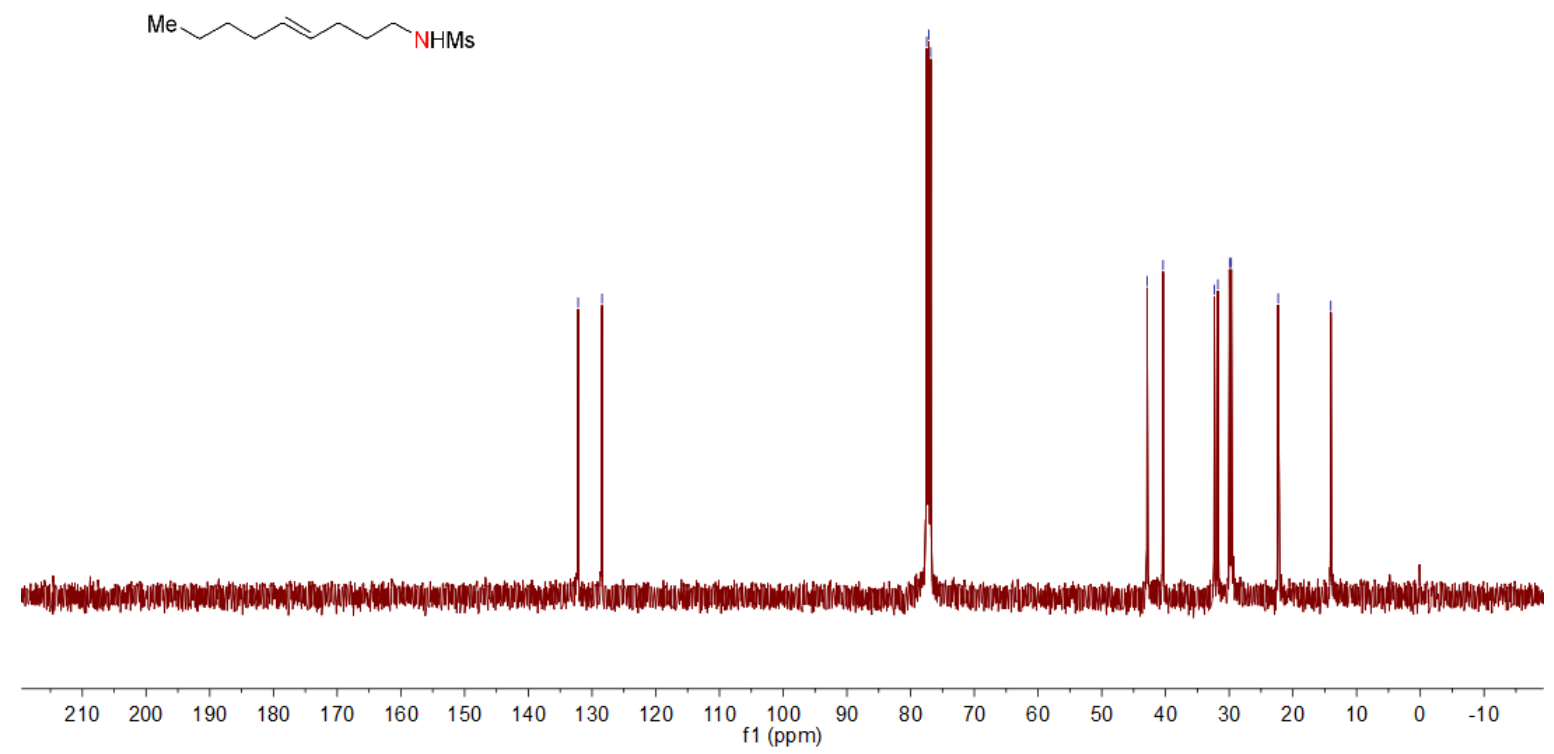


${ }^{1} \mathrm{H}$ NMR (400 MHz, $\mathrm{CDCl}_{3}$ ) spectrum of 4-Nitro- $N$-(5-( $\boldsymbol{p}$-tolyl)pent-4-en-1-yl)benzenesulfonamide (1o)

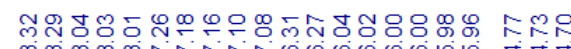

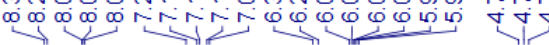
要边
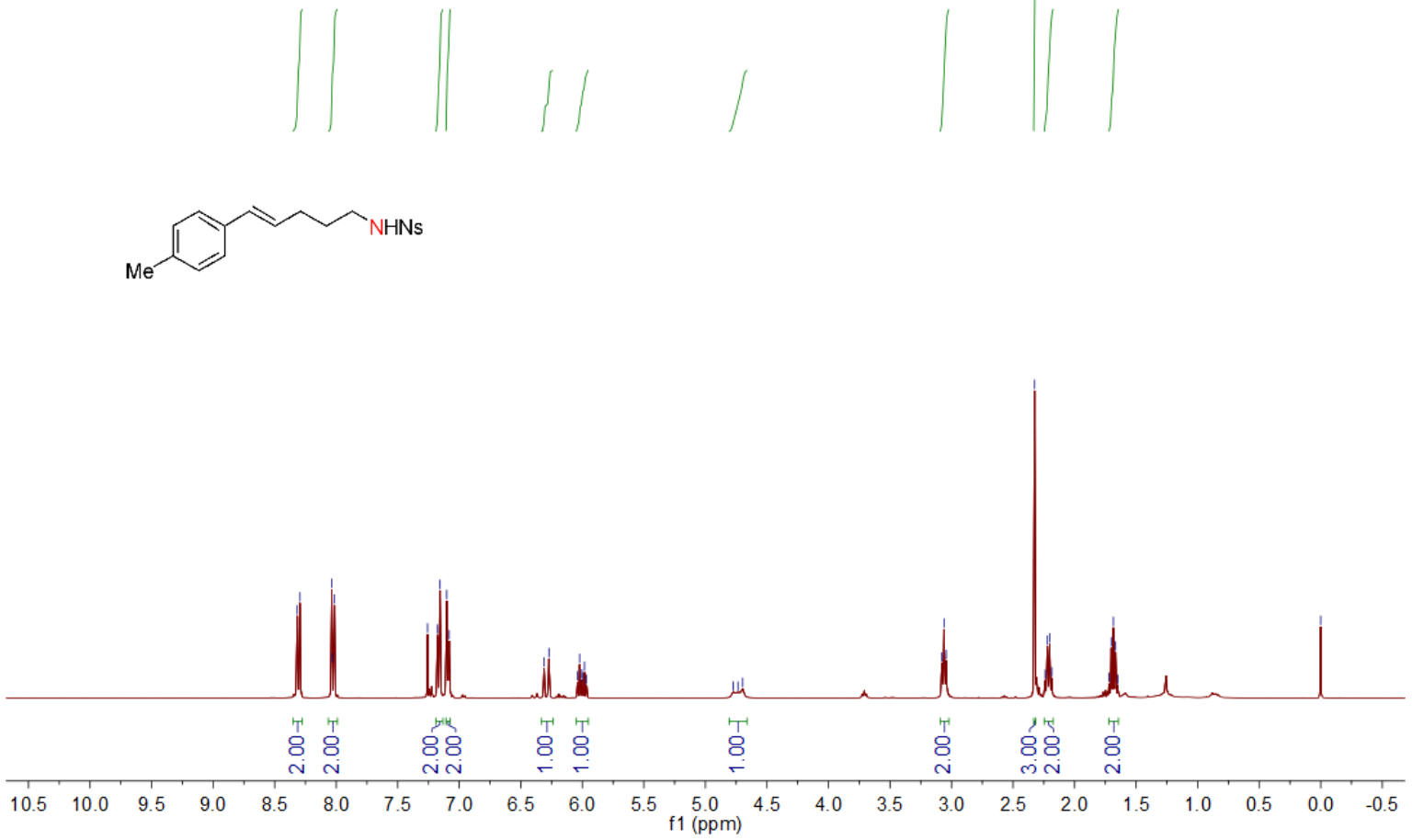

${ }^{13} \mathrm{C}$ NMR (101 MHz, $\mathrm{CDCl}_{3}$ ) spectrum of 4-Nitro- $N$-(5-(p-tolyl)pent-4-en-1-yl)benzenesulfonamide (1o)

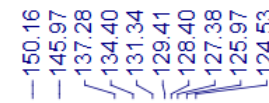

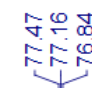

$$
\begin{aligned}
& \text { ֻु }
\end{aligned}
$$<smiles>CNCCCC=Cc1ccc(C)cc1</smiles>

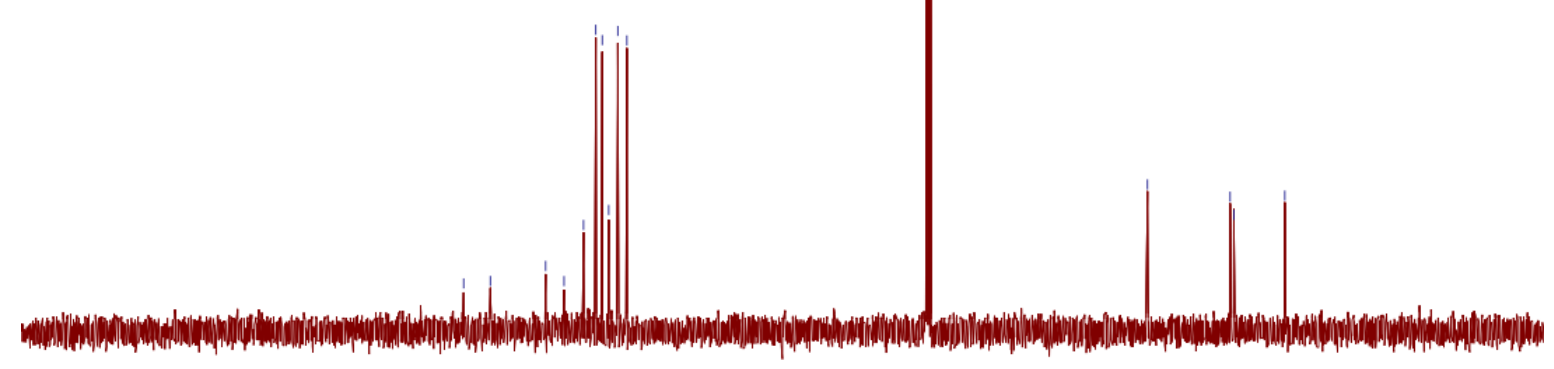

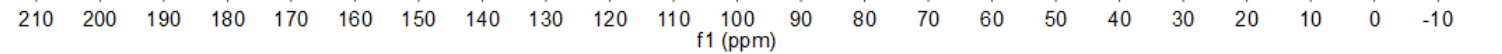


${ }^{1} \mathrm{H}$ NMR (400 MHz, $\mathrm{CDCl}_{3}$ ) spectrum of 4-Nitro- $N$-(4-phenyldec-4-en-1-yl)benzenesulfonamide (1s)

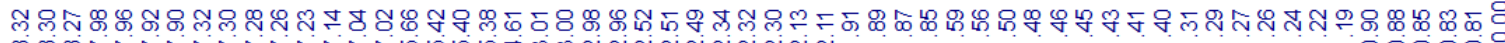

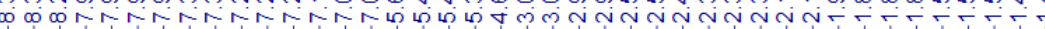

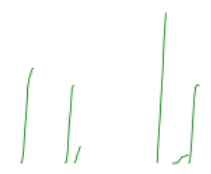<smiles>CCCCC=CC(=Cc1ccccc1)CCCNS(N)(=O)=O</smiles>

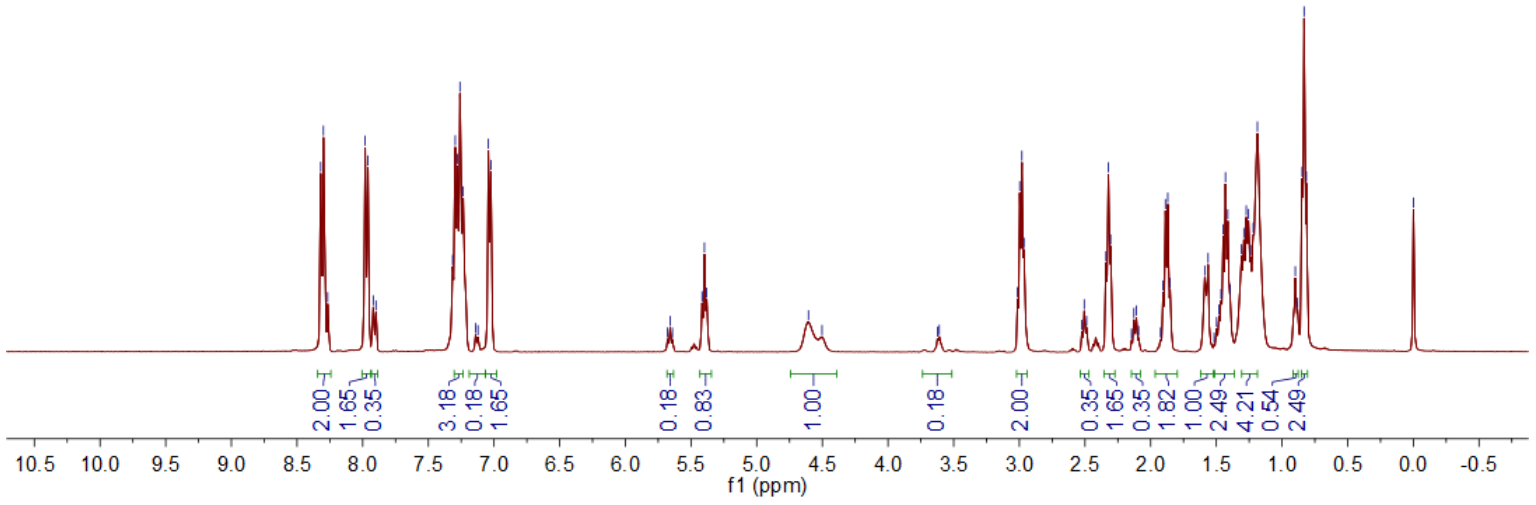

${ }^{13} \mathrm{C} \mathrm{NMR}\left(101 \mathrm{MHz}, \mathrm{CDCl}_{3}\right.$ ) spectrum of 4-Nitro- $\mathrm{N}$-(4-phenyldec-4-en-1-yl)benzenesulfonamide (1s)

$$
\text { m心in }
$$

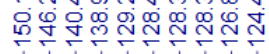

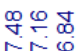

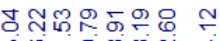

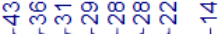<smiles>CCCCC=C(CCCNN)c1ccccc1</smiles>

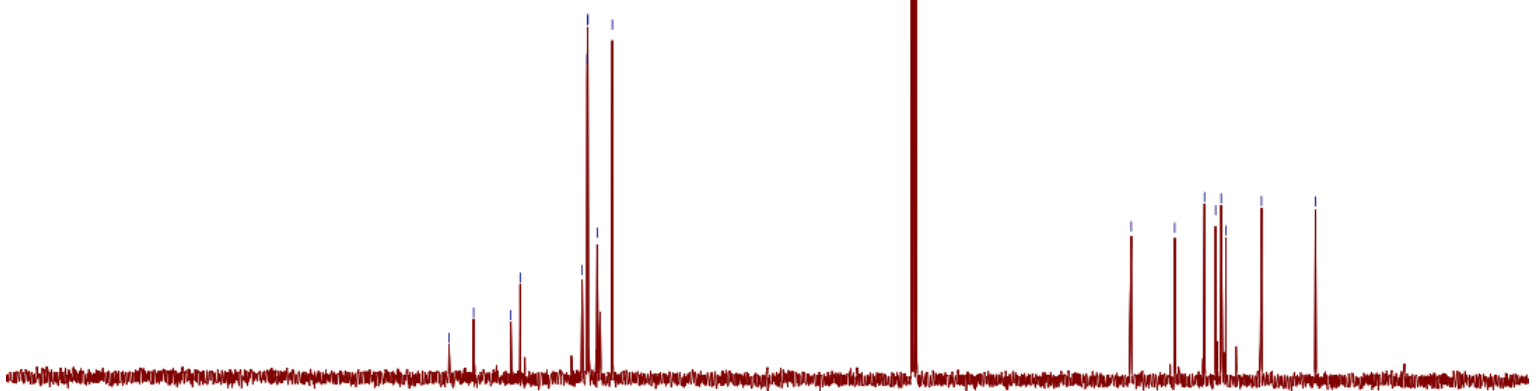

$\begin{array}{lllllllllllllllllllllll}210 & 200 & 190 & 180 & 170 & 160 & 150 & 140 & 130 & 120 & 110 & \begin{array}{l}100 \\ \mathrm{f} 1(\mathrm{ppm})\end{array} & 90 & 80 & 70 & 60 & 50 & 40 & 30 & 20 & 10 & 0 & -10\end{array}$ 
${ }^{1} \mathrm{H}$ NMR $\left(400 \mathrm{MHz}, \mathrm{CDCl}_{3}\right)$ spectrum of 1-((4-Nitrophenyl)sulfonyl)-2-(1-thiocyanatopentyl)pyrrolidine (2a)

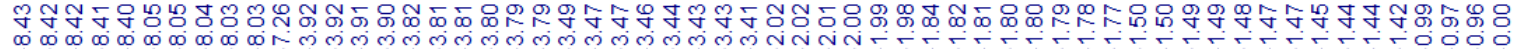

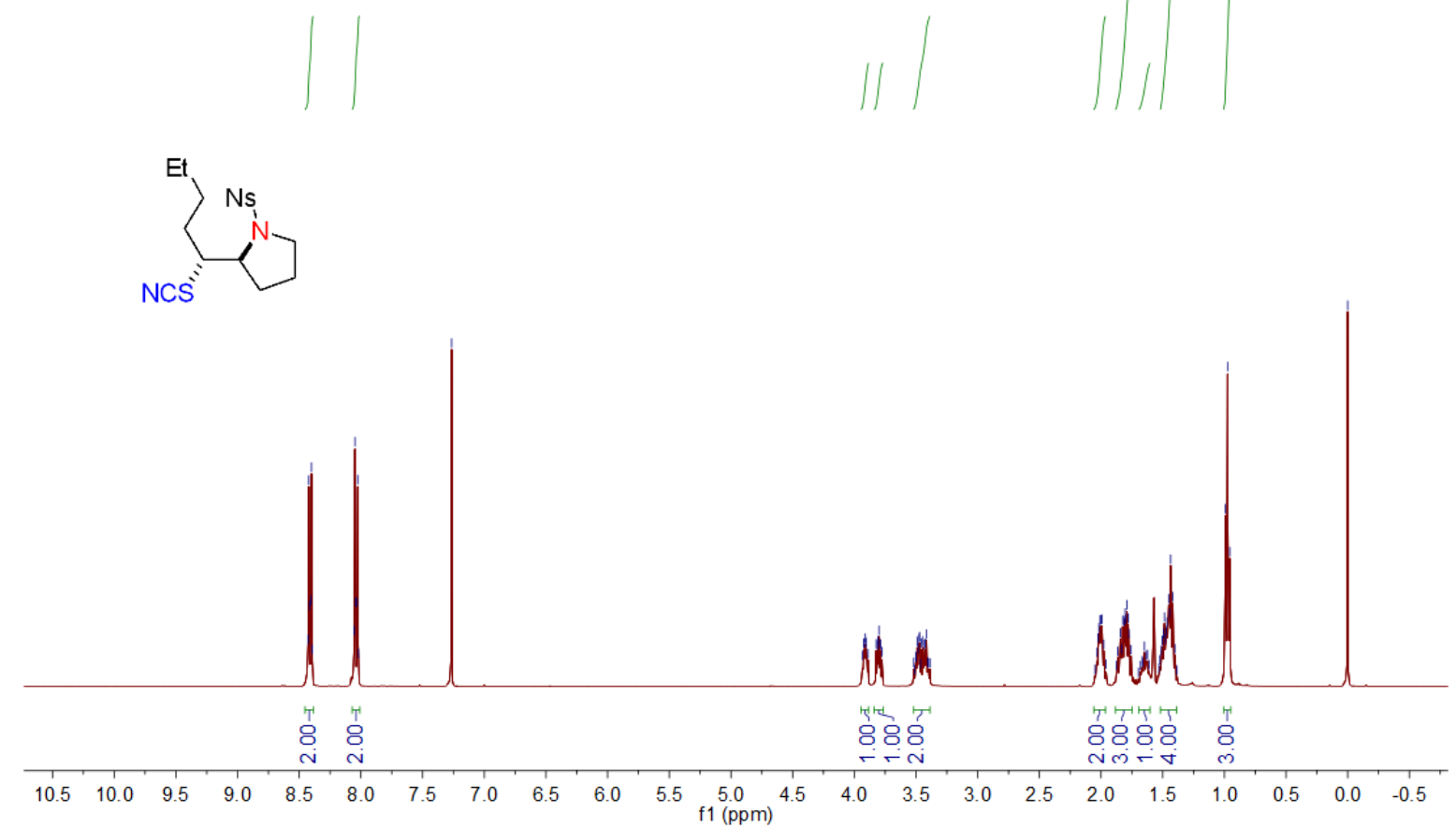

${ }^{13} \mathrm{C} \mathrm{NMR}\left(101 \mathrm{MHz}, \mathrm{CDCl}_{3}\right)$ spectrum of 1-((4-Nitrophenyl)sulfonyl)-2-(1-thiocyanatopentyl)pyrrolidine (2a)

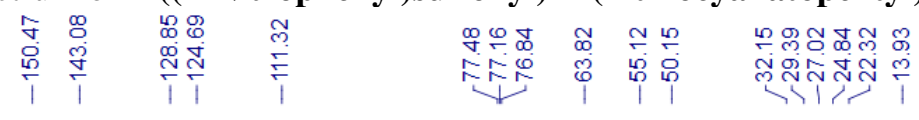

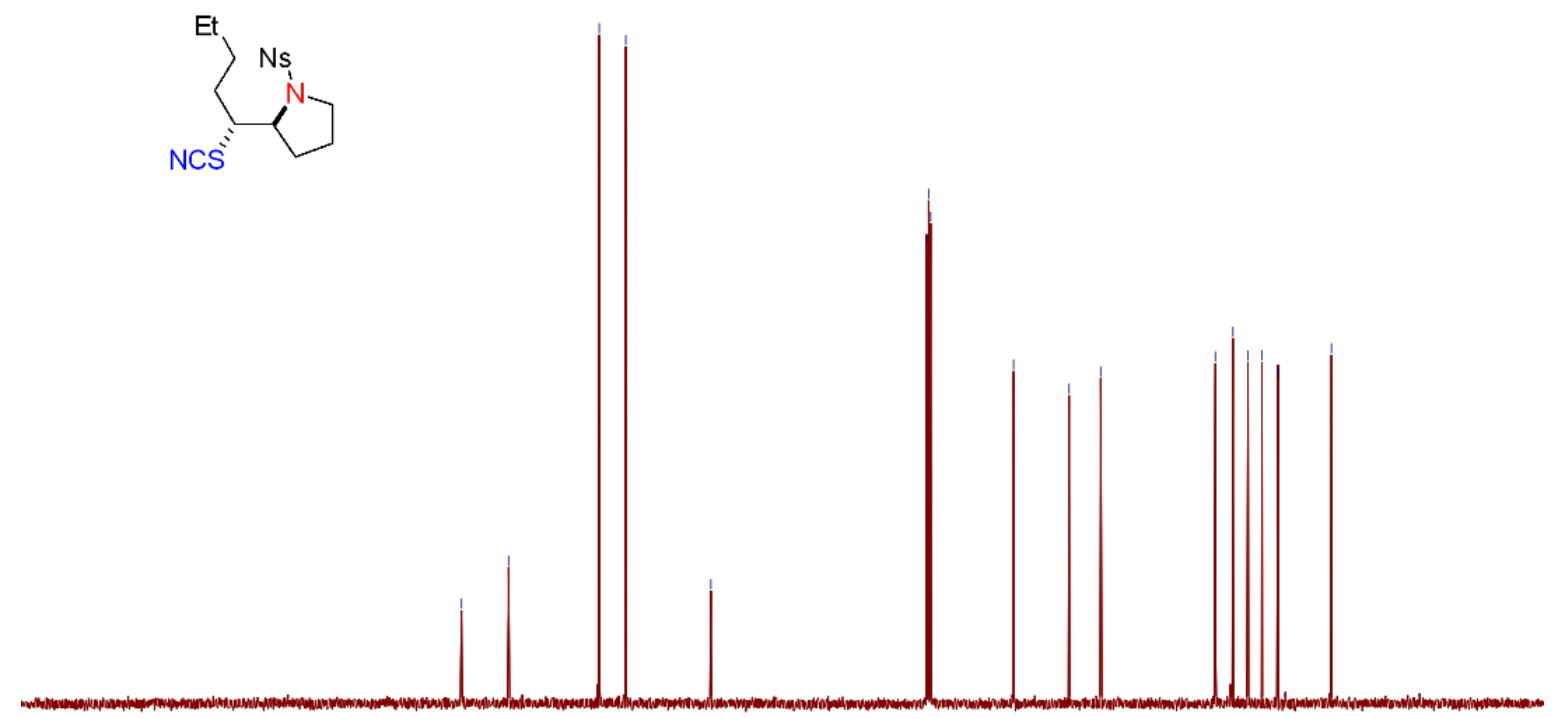

$\begin{array}{lllllllllllllllllllllllllll}210 & 200 & 190 & 180 & 170 & 160 & 150 & 140 & 130 & 120 & 110 & \begin{array}{c}100 \\ \mathrm{f} 1(\mathrm{ppm})\end{array} & 90 & 80 & 70 & 60 & 50 & 40 & 30 & 20 & 10 & 0 & -10\end{array}$ 
${ }^{1} \mathrm{H}$ NMR (400 MHz, $\mathrm{CDCl}_{3}$ ) spectrum of 2-Butyl-1-((4-nitrophenyl)sulfonyl)-3-thiocyanatopiperidine (3a)

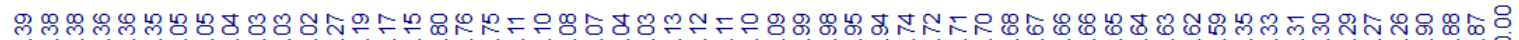

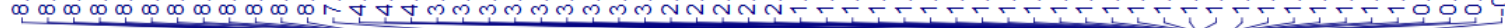<smiles>CCCC1C(C#N)CCCN1N</smiles>

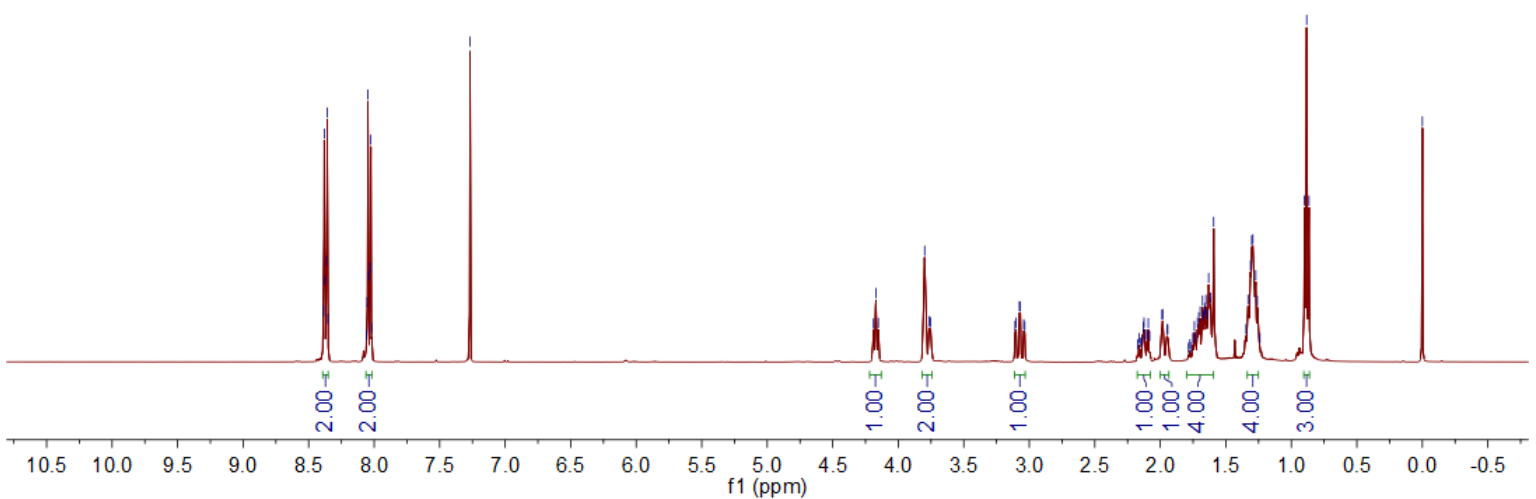

${ }^{13} \mathrm{C}$ NMR (101 MHz, $\mathrm{CDCl}_{3}$ ) spectrum of 2-Butyl-1-((4-nitrophenyl)sulfonyl)-3-thiocyanatopiperidine (3a)

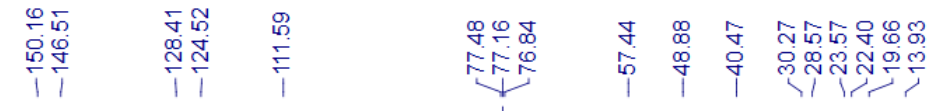

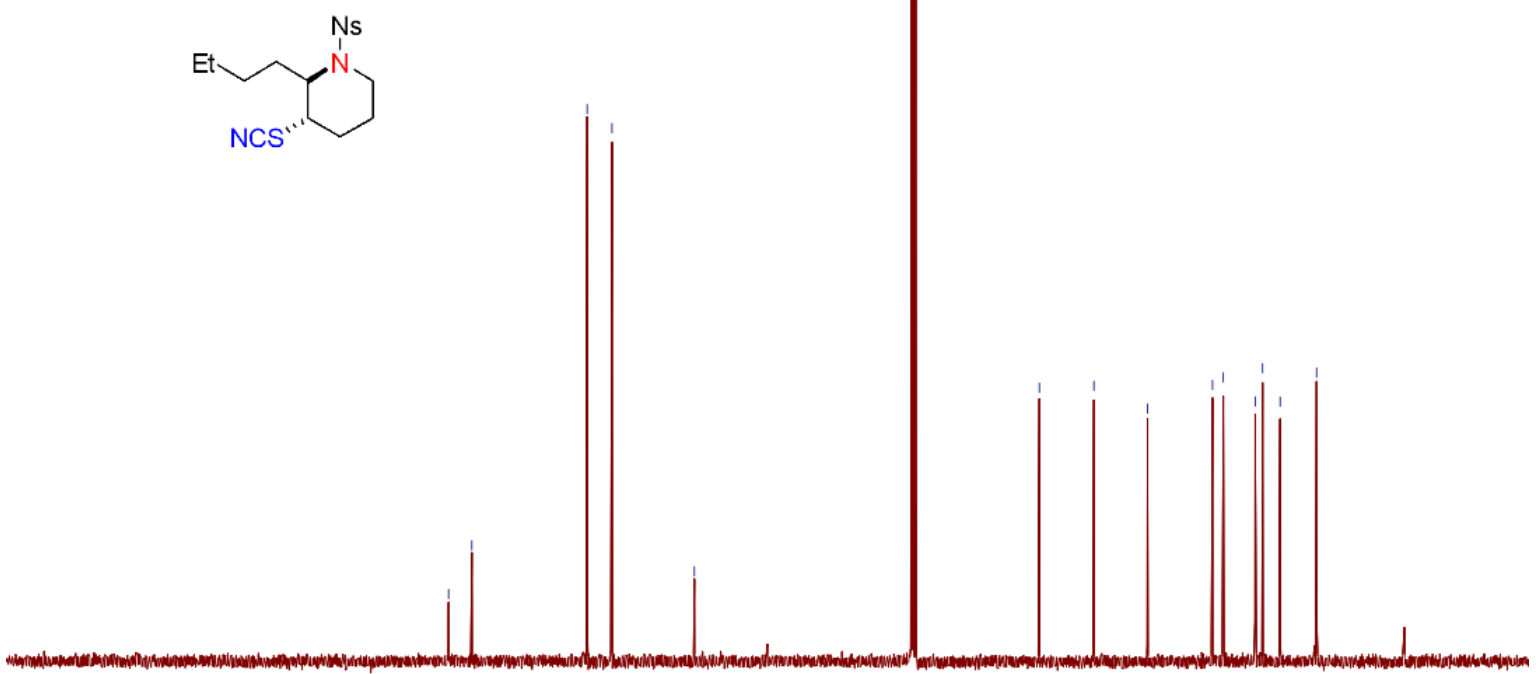

$\begin{array}{llllllllllllllllllllll}210 & 200 & 190 & 180 & 170 & 160 & 150 & 140 & 130 & 120 & 110 \begin{array}{c}100 \\ \mathrm{f} 1(\mathrm{ppm})\end{array} & 90 & 80 & 70 & 60 & 50 & 40 & 30 & 20 & 10 & 0 & -10\end{array}$ 
${ }^{1} \mathrm{H} \mathrm{NMR}\left(400 \mathrm{MHz}, \mathrm{CDCl}_{3}\right)$ spectrum of 1-((4-Nitrophenyl)sulfonyl)-2-(1-thiocyanatopropyl)pyrrolidine (2b)

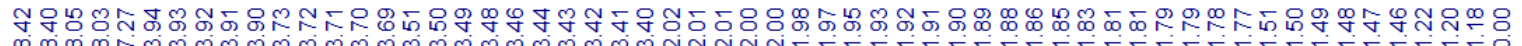

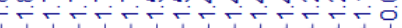

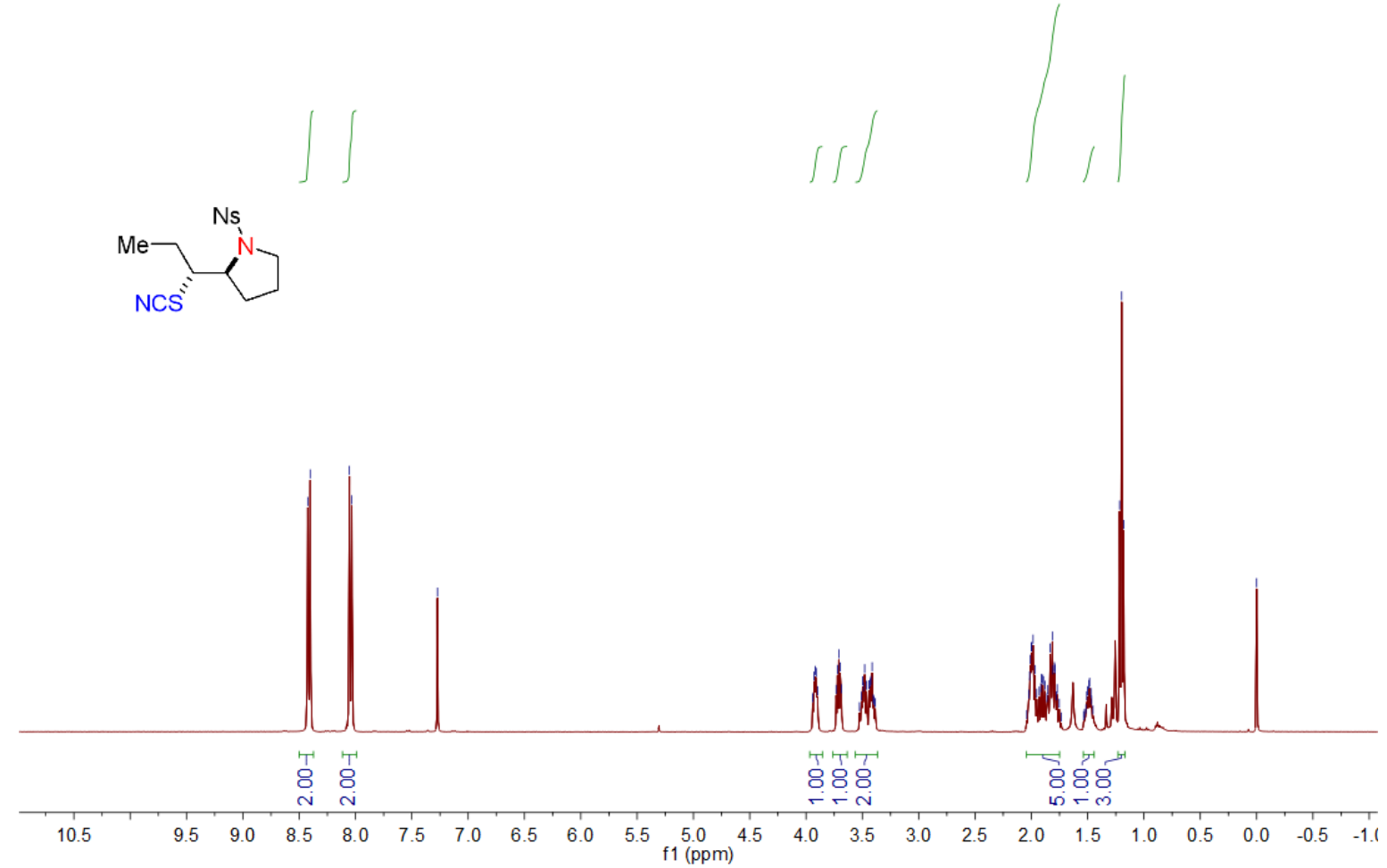

${ }^{13} \mathrm{C}$ NMR (101 MHz, $\left.\mathrm{CDCl}_{3}\right)$ spectrum of 1-((4-Nitrophenyl)sulfonyl)-2-(1-thiocyanatopropyl)pyrrolidine (2b)

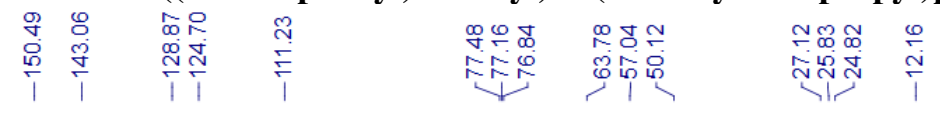

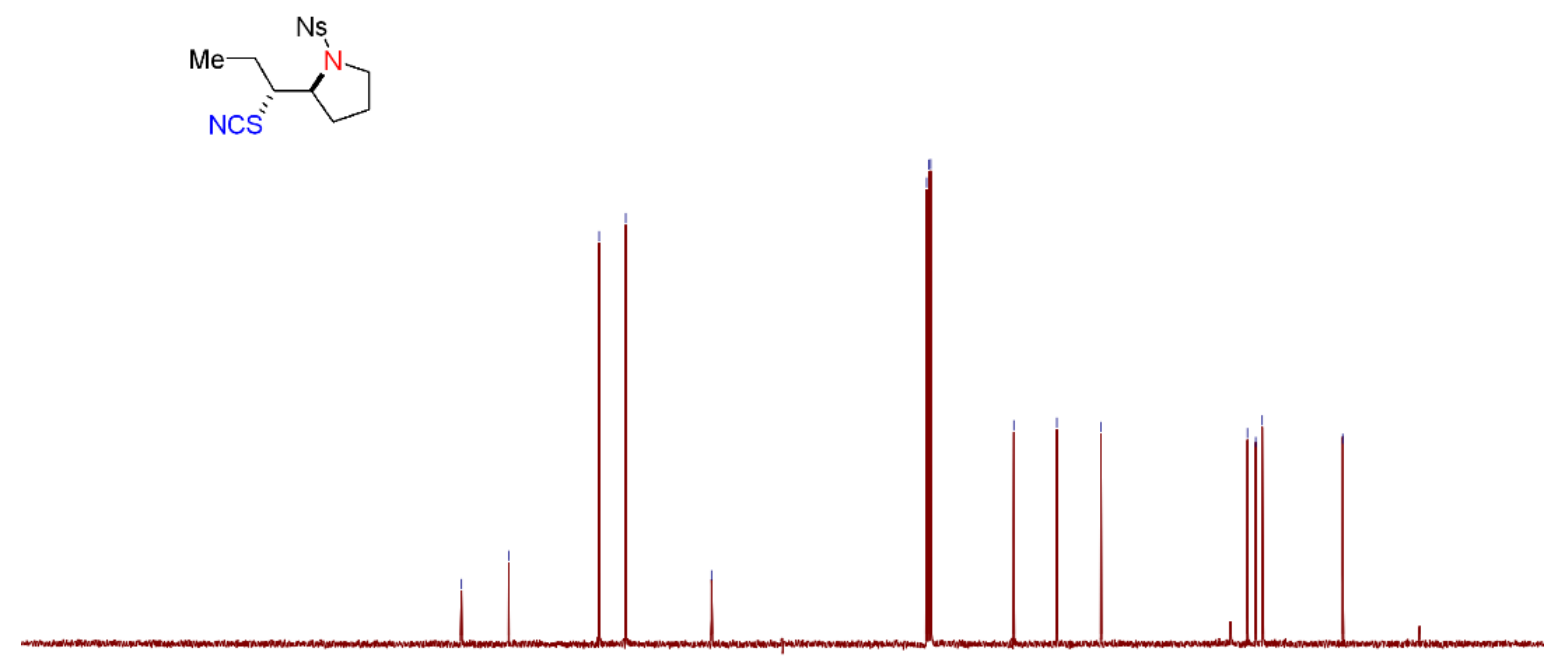

$\begin{array}{llllllllllllllllllllll}210 & 200 & 190 & 180 & 170 & 160 & 150 & 140 & 130 & 120 & 110 \begin{array}{c}100 \\ \mathrm{f} 1(\mathrm{ppm})\end{array} & 90 & 80 & 70 & 60 & 50 & 40 & 30 & 20 & 10 & 0 & -10\end{array}$ 
${ }^{1} \mathrm{H} \quad \mathrm{NMR} \quad\left(400 \mathrm{MHz}, \mathrm{CDCl}_{3}\right) \quad$ spectrum of $\boldsymbol{r e l - ( S ) - 1 - ( ( 4 - N i t r o p h e n y l ) s u l f o n y l ) - 2 - ( ( \boldsymbol { R } ) - 1 - t h i o c y a n a t o e t h y l )}$ pyrrolidine (2c)
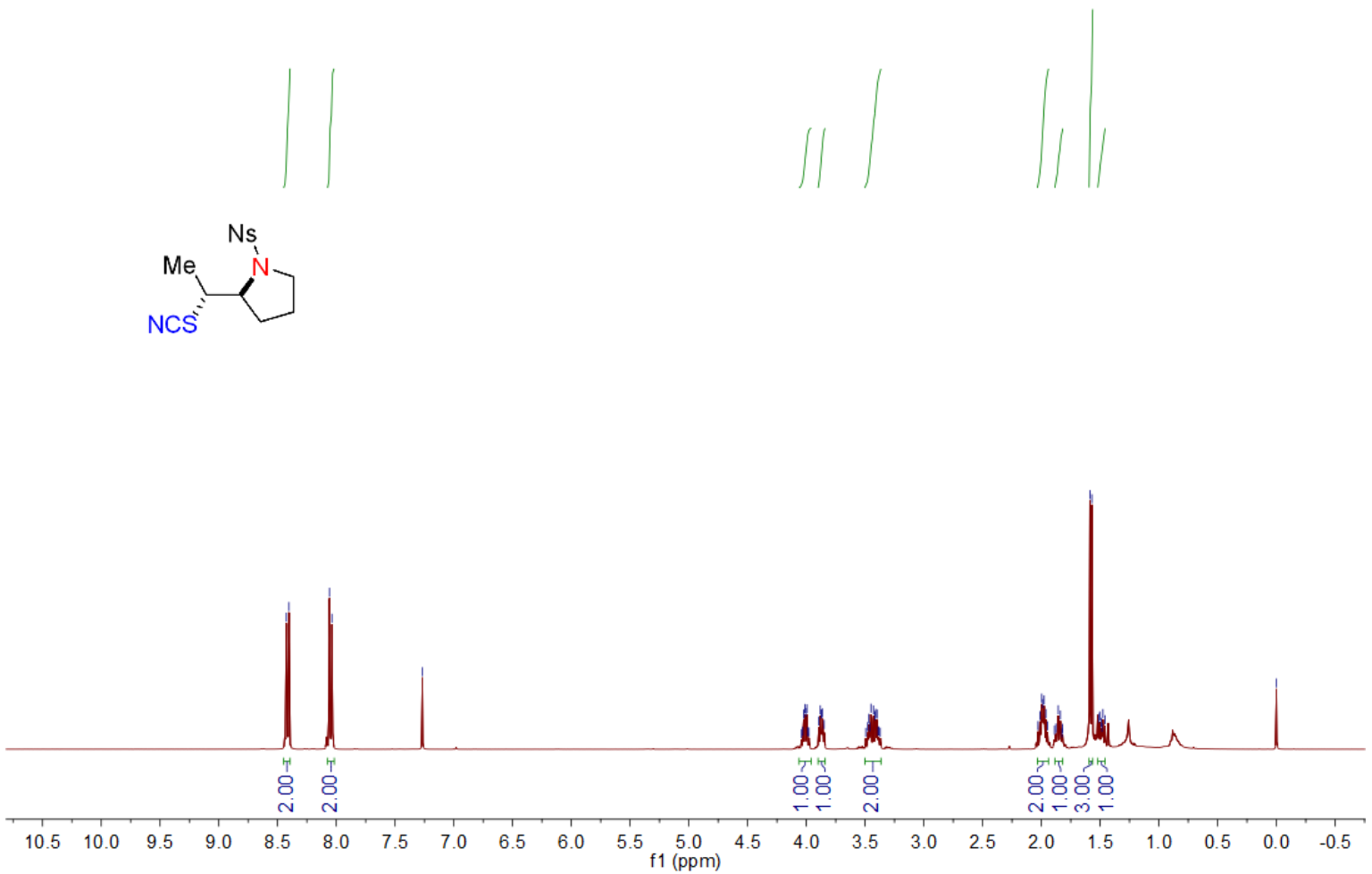

${ }^{13} \mathrm{C} \quad \mathrm{NMR}\left(101 \mathrm{MHz}, \mathrm{CDCl}_{3}\right)$ spectrum of $\boldsymbol{r e l}$-(S)-1-((4-Nitrophenyl)sulfonyl)-2-((R)-1-thiocyanatoethyl) pyrrolidine (2c)

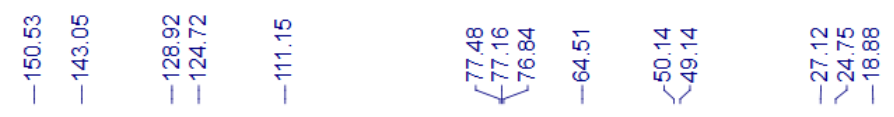
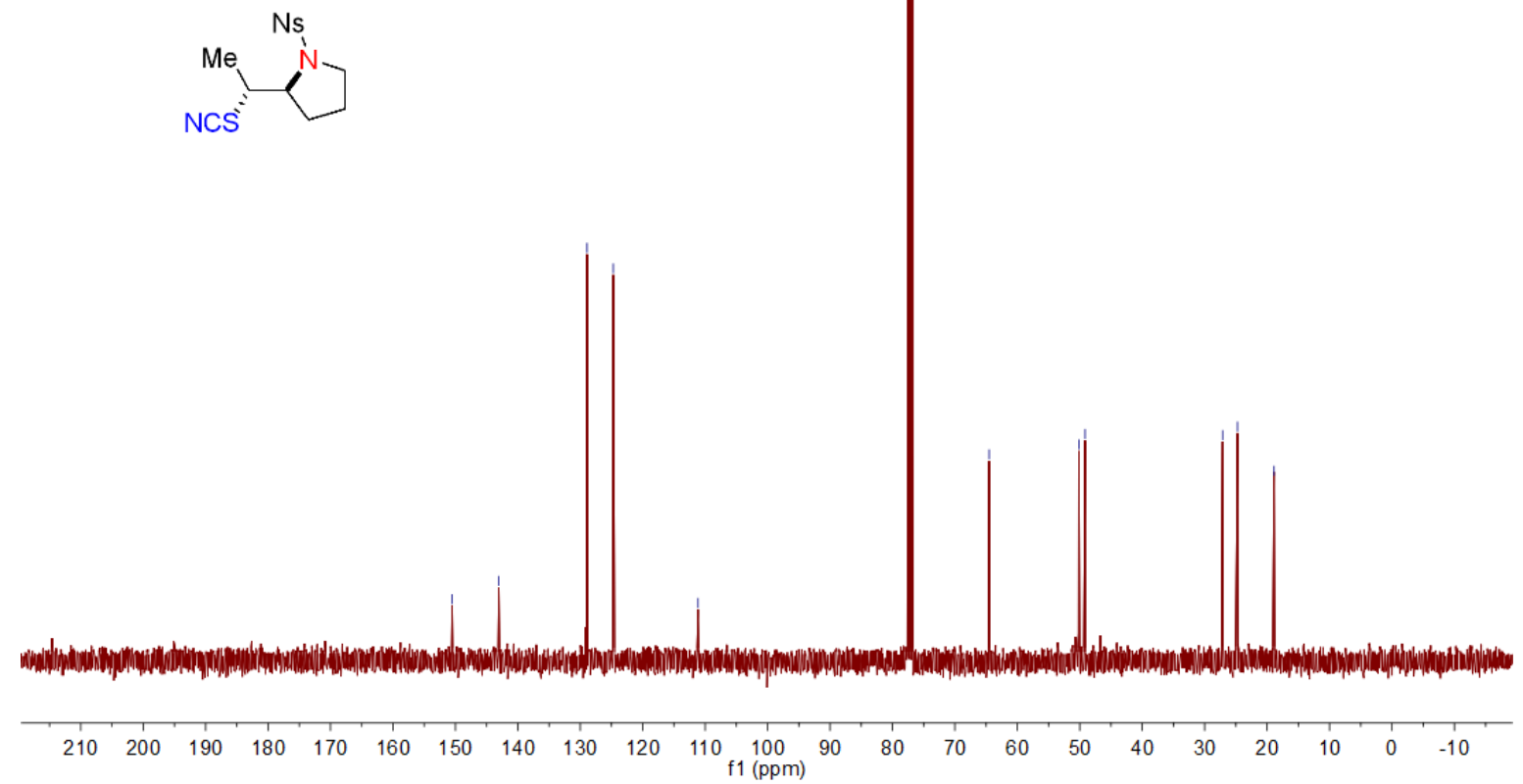
${ }^{1} \mathrm{H} \quad \mathrm{NMR} \quad\left(400 \mathrm{MHz}, \mathrm{CDCl}_{3}\right)$ spectrum of 2-(5-Chloro-1-thiocyanatopentyl)-1-((4-nitrophenyl)sulfonyl) pyrrolidine (2d)

궉ㅇำ

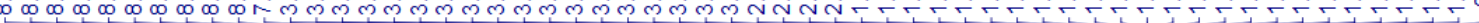
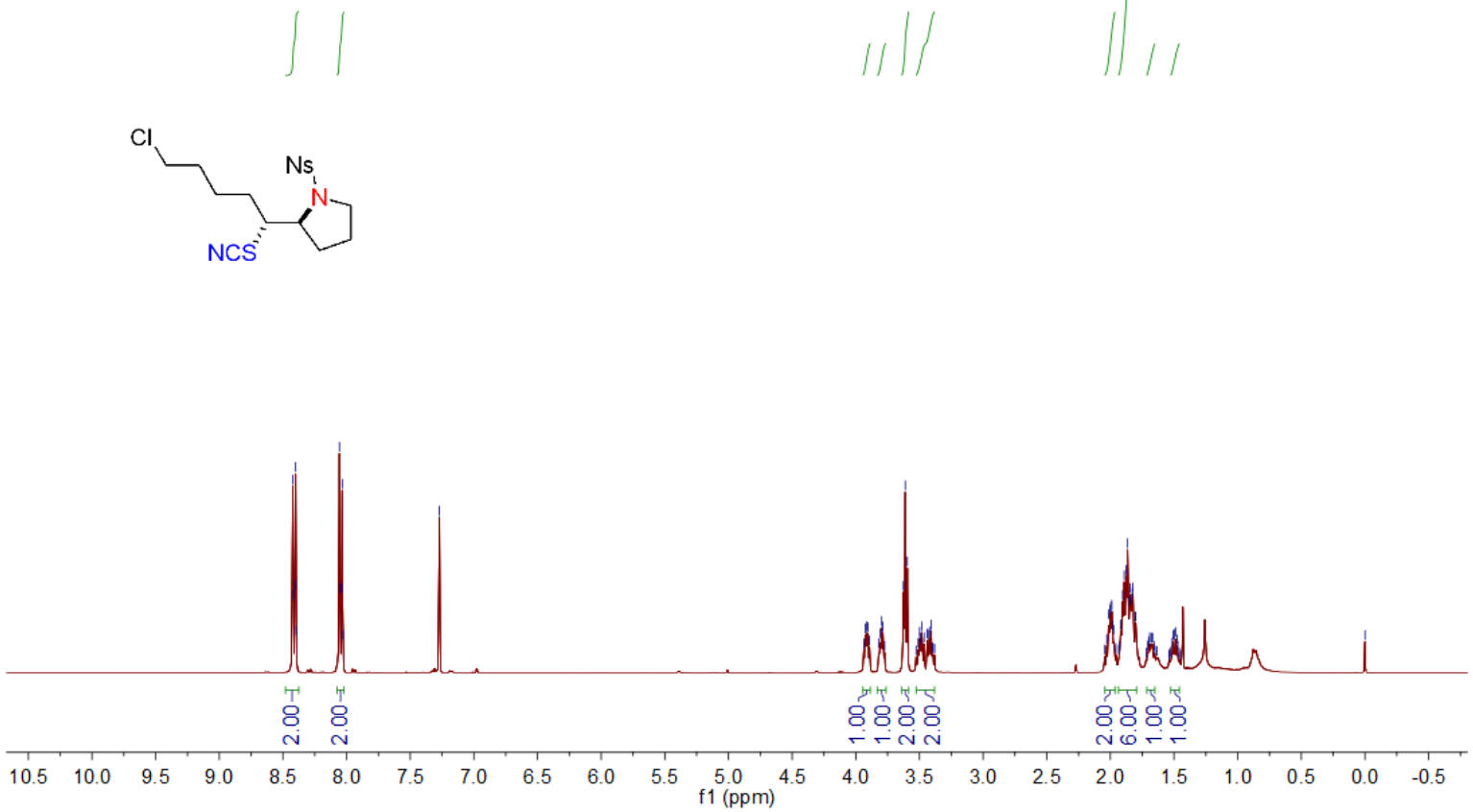

${ }^{13} \mathrm{C}$ NMR (101 $\left.\mathrm{MHz}, \mathrm{CDCl}_{3}\right)$ spectrum of 2-(5-Chloro-1-thiocyanatopentyl)-1-((4-nitrophenyl)sulfonyl) pyrrolidine (2d)
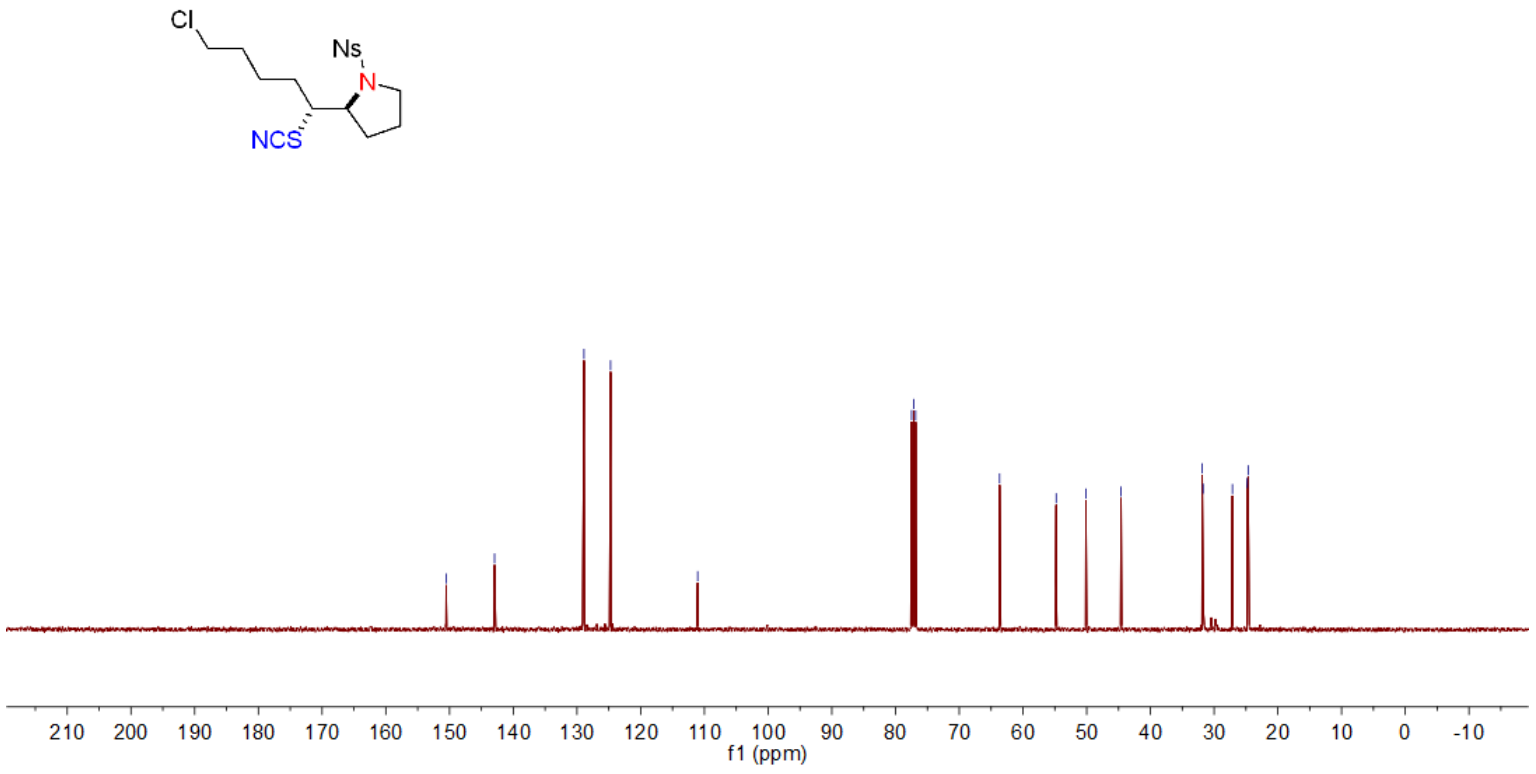
${ }^{1} \mathrm{H} \quad \mathrm{NMR} \quad\left(400 \mathrm{MHz}, \mathrm{CDCl}_{3}\right)$ spectrum of 2-(3-Methyl-1-thiocyanatobutyl)-1-((4-nitrophenyl)sulfonyl) pyrrolidine (2e)

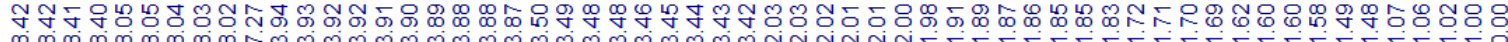
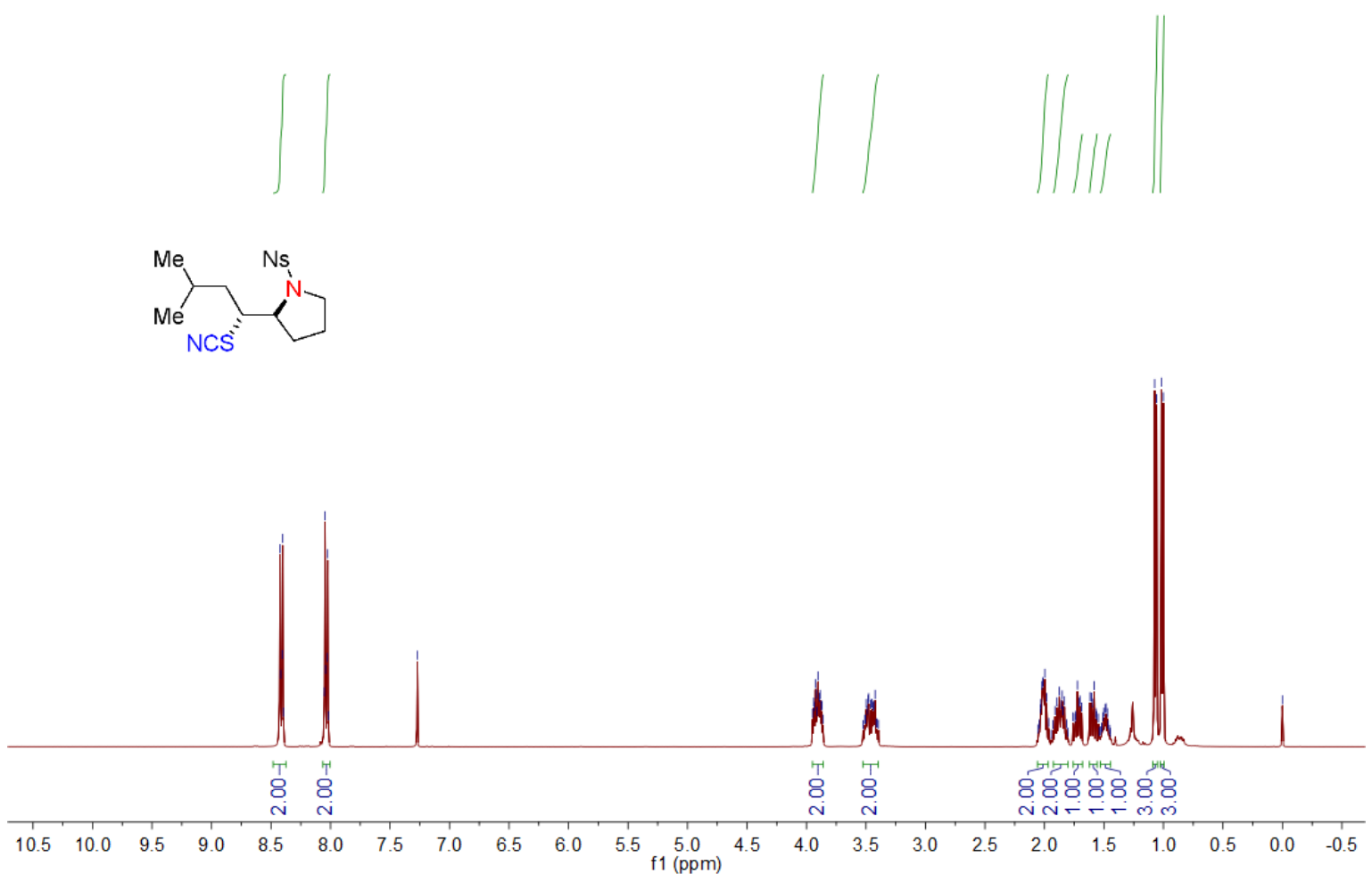

${ }^{13} \mathrm{C} \quad \mathrm{NMR} \quad\left(101 \mathrm{MHz}, \mathrm{CDCl}_{3}\right)$ spectrum of 2-(3-Methyl-1-thiocyanatobutyl)-1-((4-nitrophenyl)sulfonyl) pyrrolidine (2e)

$$
\text { 占 }
$$

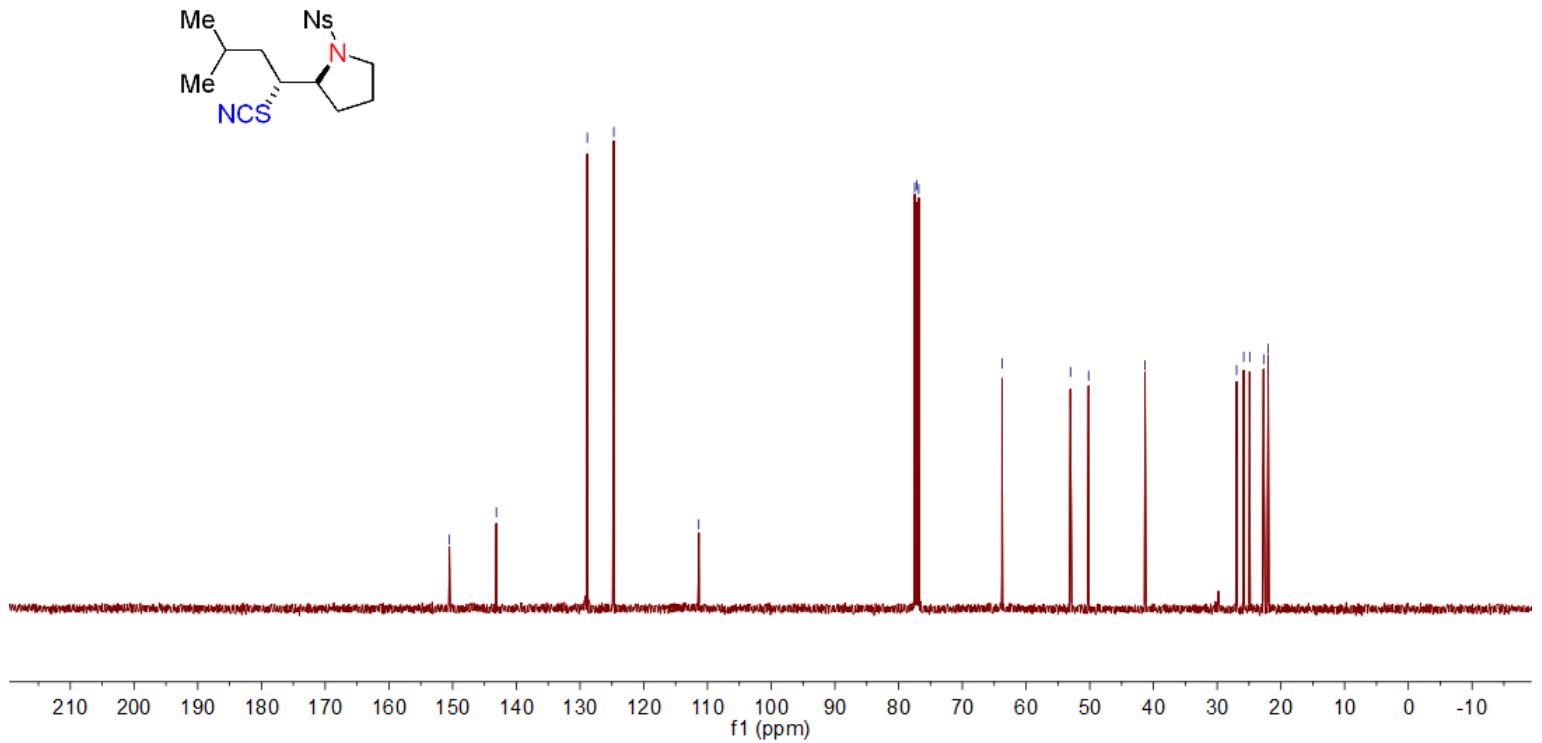


${ }^{1} \mathrm{H}$ NMR (400 MHz, $\mathrm{CDCl}_{3}$ ) spectrum of 2-(2-Ethyl-1-thiocyanatohexyl)-1-((4-nitrophenyl)sulfonyl)pyrrolidine (2f)

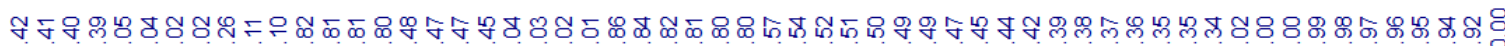

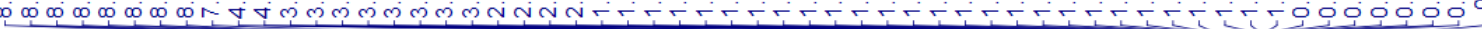
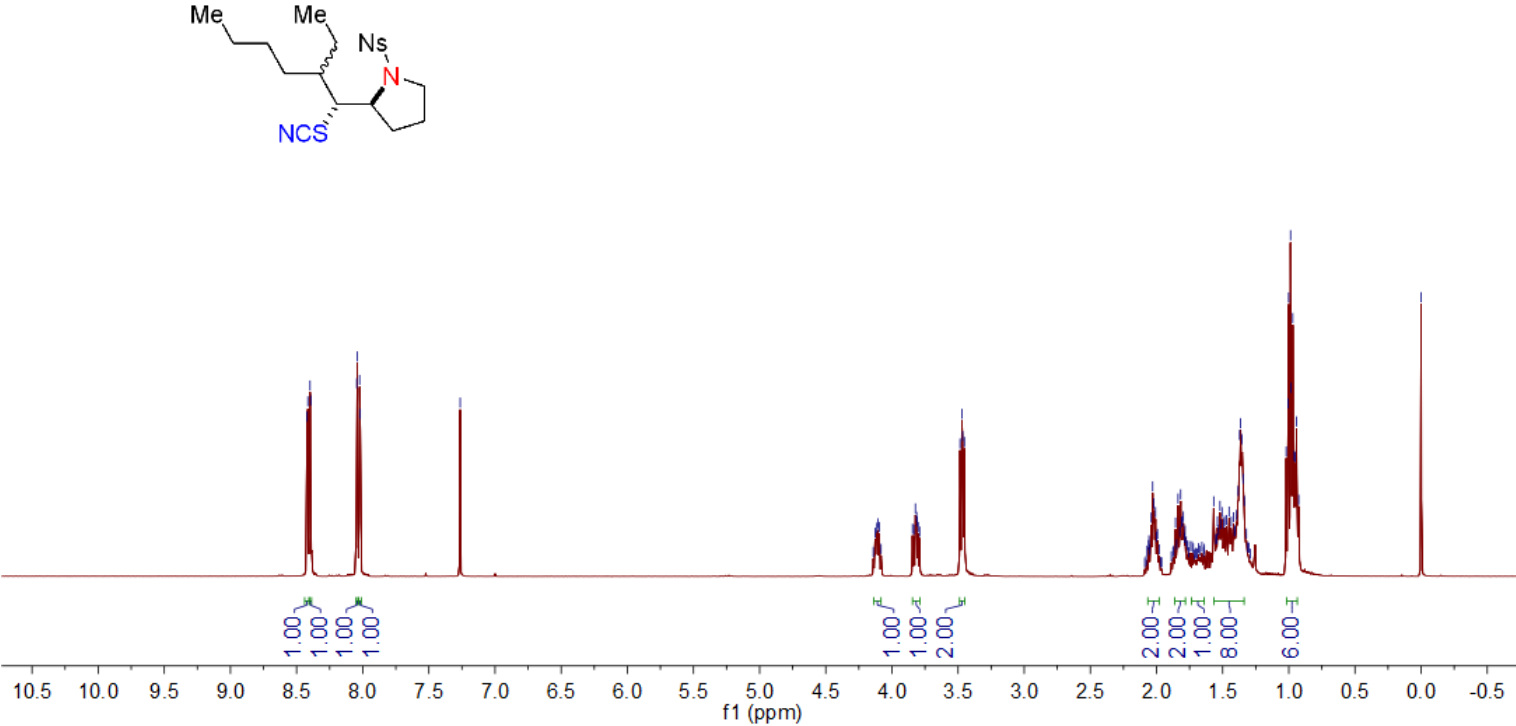

${ }^{13} \mathrm{C} \quad \mathrm{NMR} \quad\left(101 \mathrm{MHz}, \mathrm{CDCl}_{3}\right) \quad$ spectrum of 2-(2-Ethyl-1-thiocyanatohexyl)-1-((4-nitrophenyl)sulfonyl) pyrrolidine (2f)
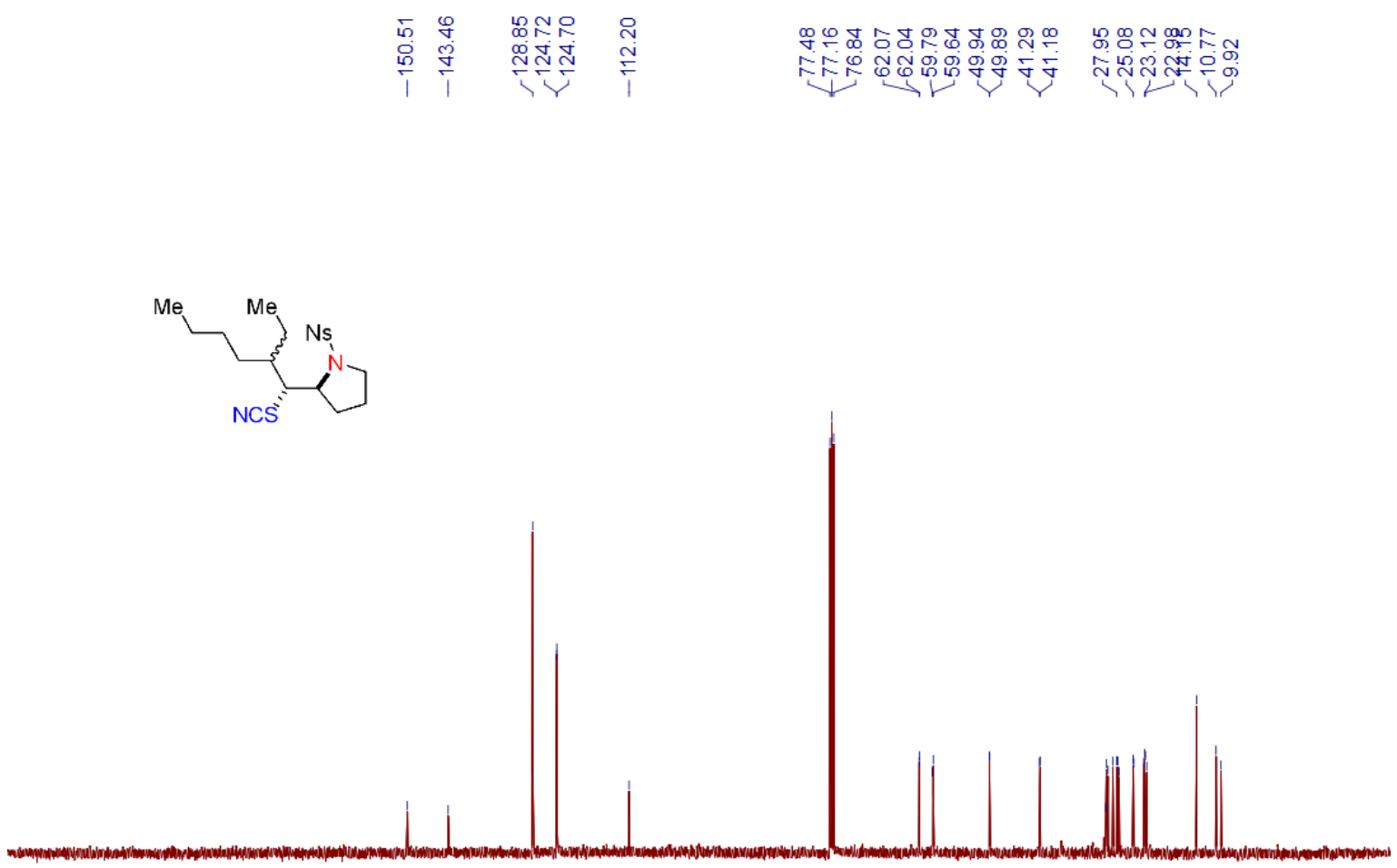

$\begin{array}{lllllllllllllllllllllll}210 & 200 & 190 & 180 & 170 & 160 & 150 & 140 & 130 & 120 & 110 & 100 & 90 & 80 & 70 & 60 & 50 & 40 & 30 & 20 & 10 & 0 & -10\end{array}$ 
${ }^{1} \mathrm{H}$ NMR (400 $\left.\mathrm{MHz}, \mathrm{CDCl}_{3}\right)$ spectrum of 2-(Cyclohexyl(thiocyanato)methyl)-1-((4-nitrophenyl)sulfonyl) pyrrolidine (2g)

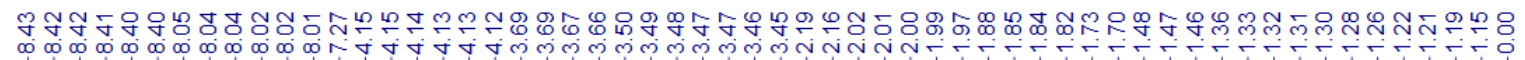
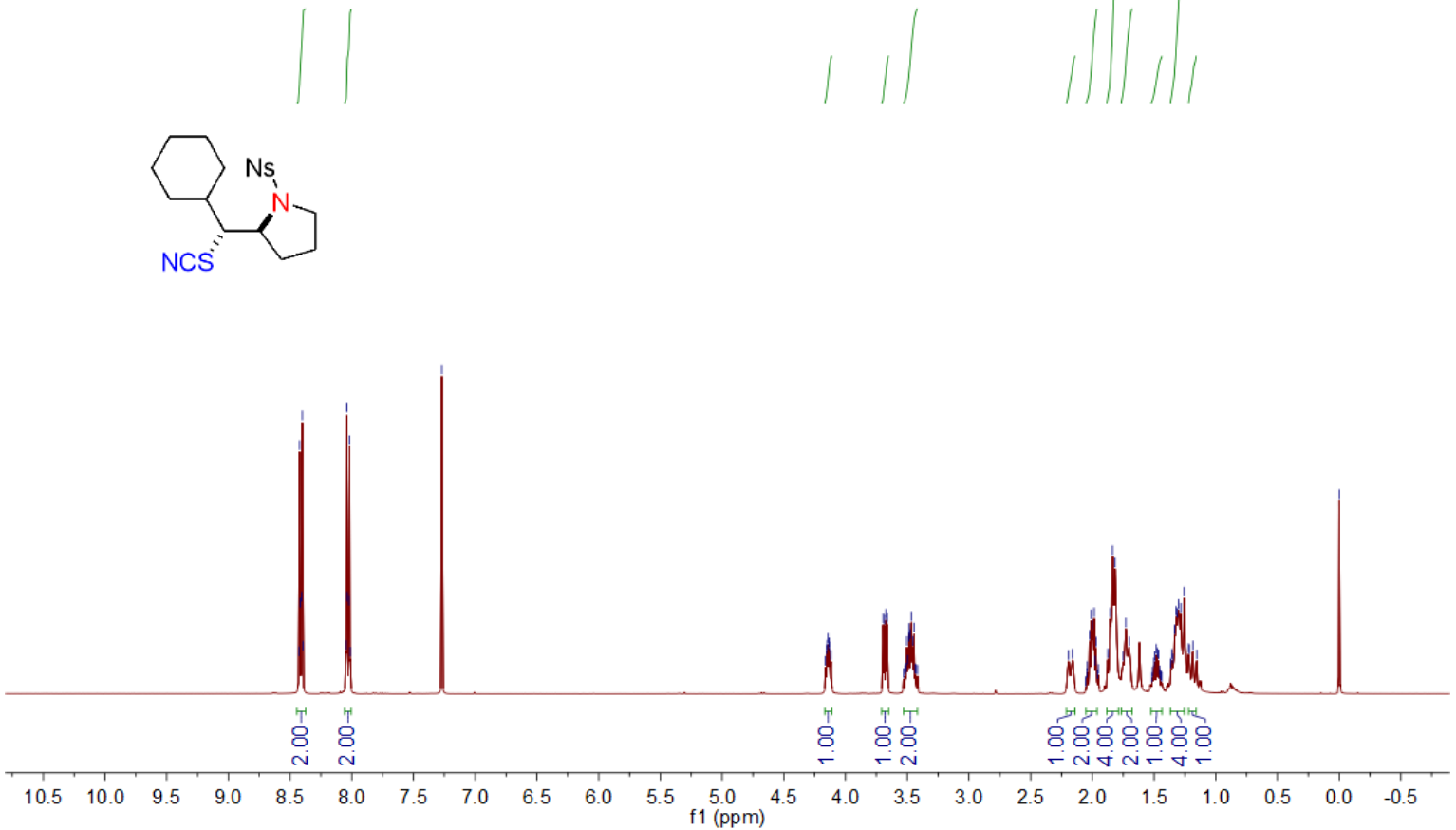

${ }^{13} \mathrm{C}$ NMR (101 $\left.\mathrm{MHz}, \mathrm{CDCl}_{3}\right)$ spectrum of 2-(Cyclohexyl(thiocyanato)methyl)-1-((4-nitrophenyl)sulfonyl) pyrrolidine (2g)

li

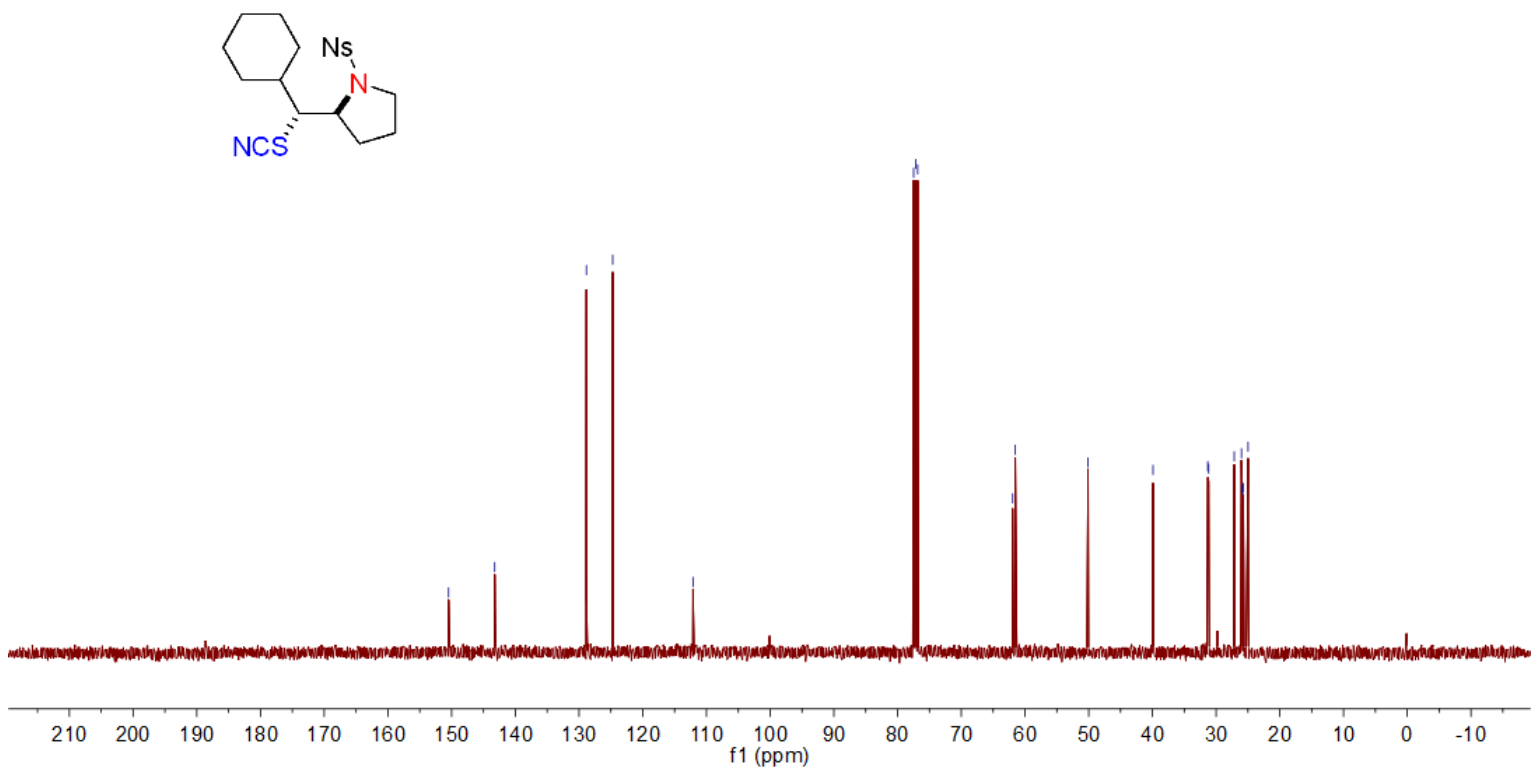


${ }^{1} \mathrm{H}$ NMR (400 MHz, $\mathrm{CDCl}_{3}$ ) spectrum of 2-(2,2-Dimethyl-1-thiocyanatopropyl)-1-((4-nitrophenyl)sulfonyl) pyrrolidine (2h)

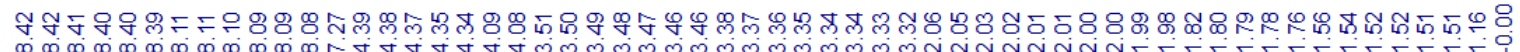
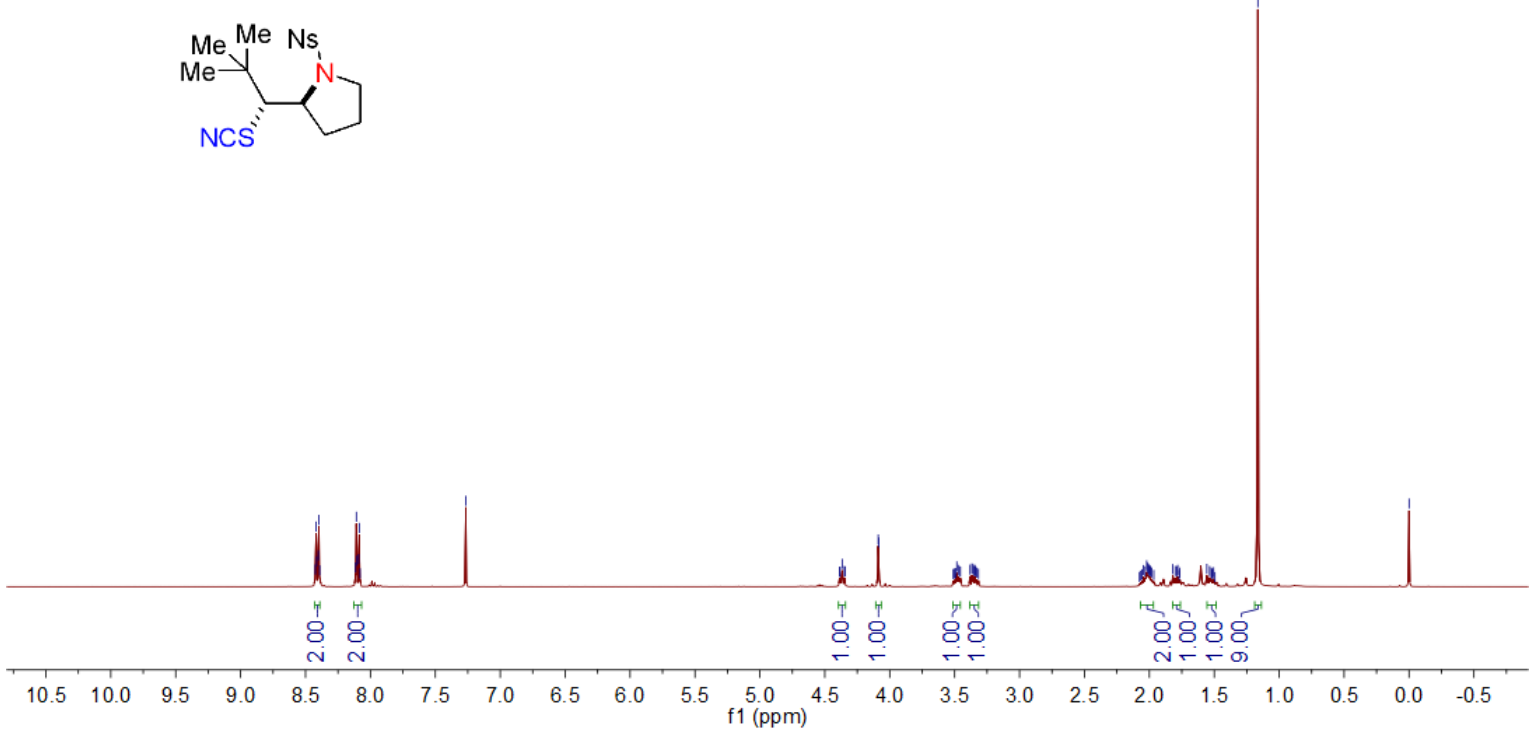

${ }^{13} \mathrm{C} \quad \mathrm{NMR} \quad\left(101 \mathrm{MHz}, \quad \mathrm{CDCl}_{3}\right) \quad$ spectrum of 2-(2,2-Dimethyl-1-thiocyanatopropyl)-1-((4-nitrophenyl) sulfonyl)pyrrolidine (2h)

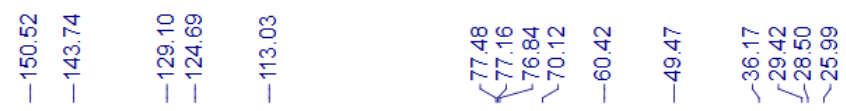

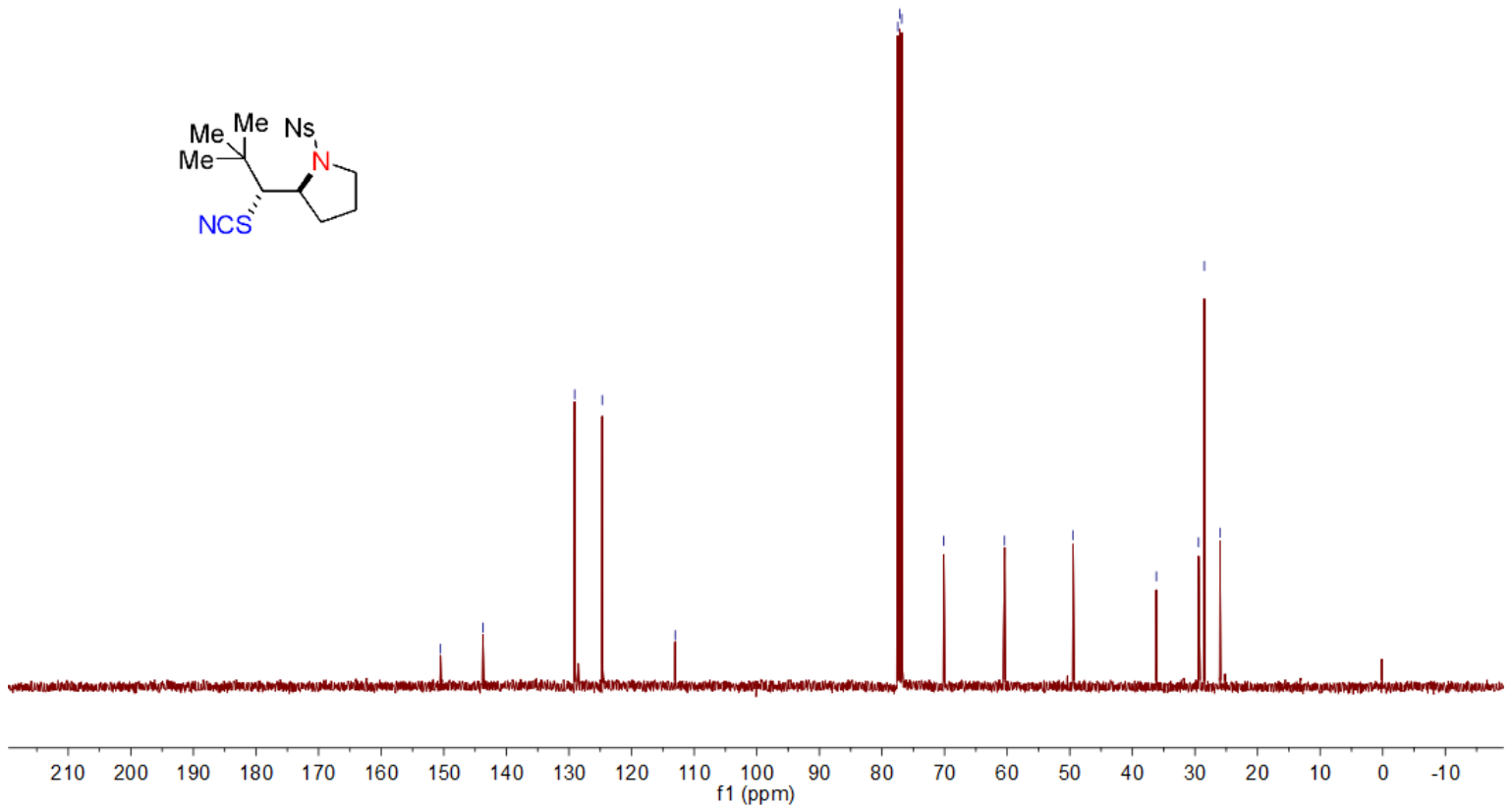


${ }^{1} \mathrm{H}$ NMR (400 MHz, $\left.\mathrm{CDCl}_{3}\right)$ spectrum of 1-((4-Nitrophenyl)sulfonyl)-2-(thiocyanatomethyl)pyrrolidine (2i)
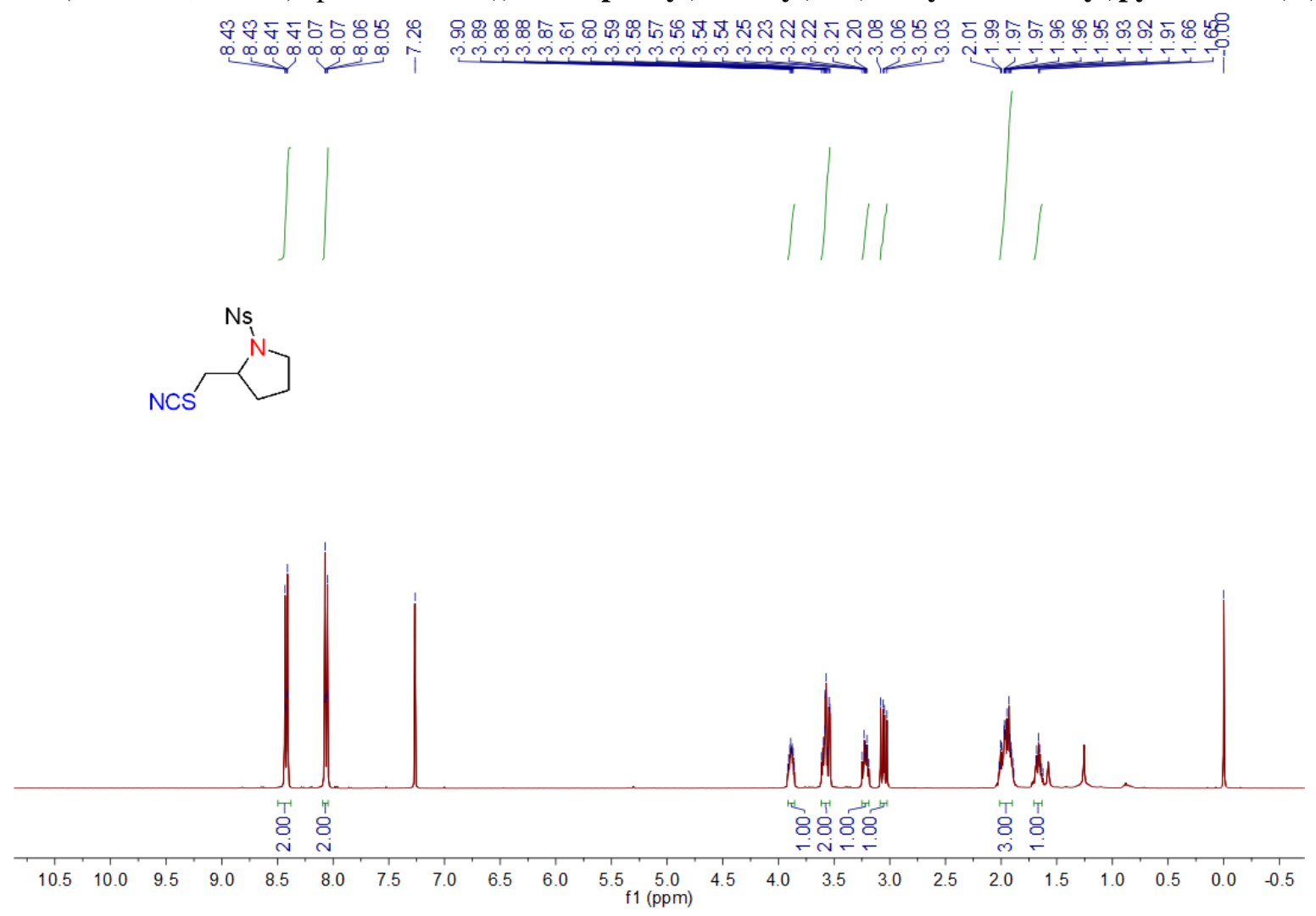

${ }^{13} \mathrm{C}$ NMR (101 MHz, $\left.\mathrm{CDCl}_{3}\right)$ spectrum of 1-((4-Nitrophenyl)sulfonyl)-2-(thiocyanatomethyl)pyrrolidine (2i)

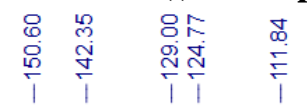
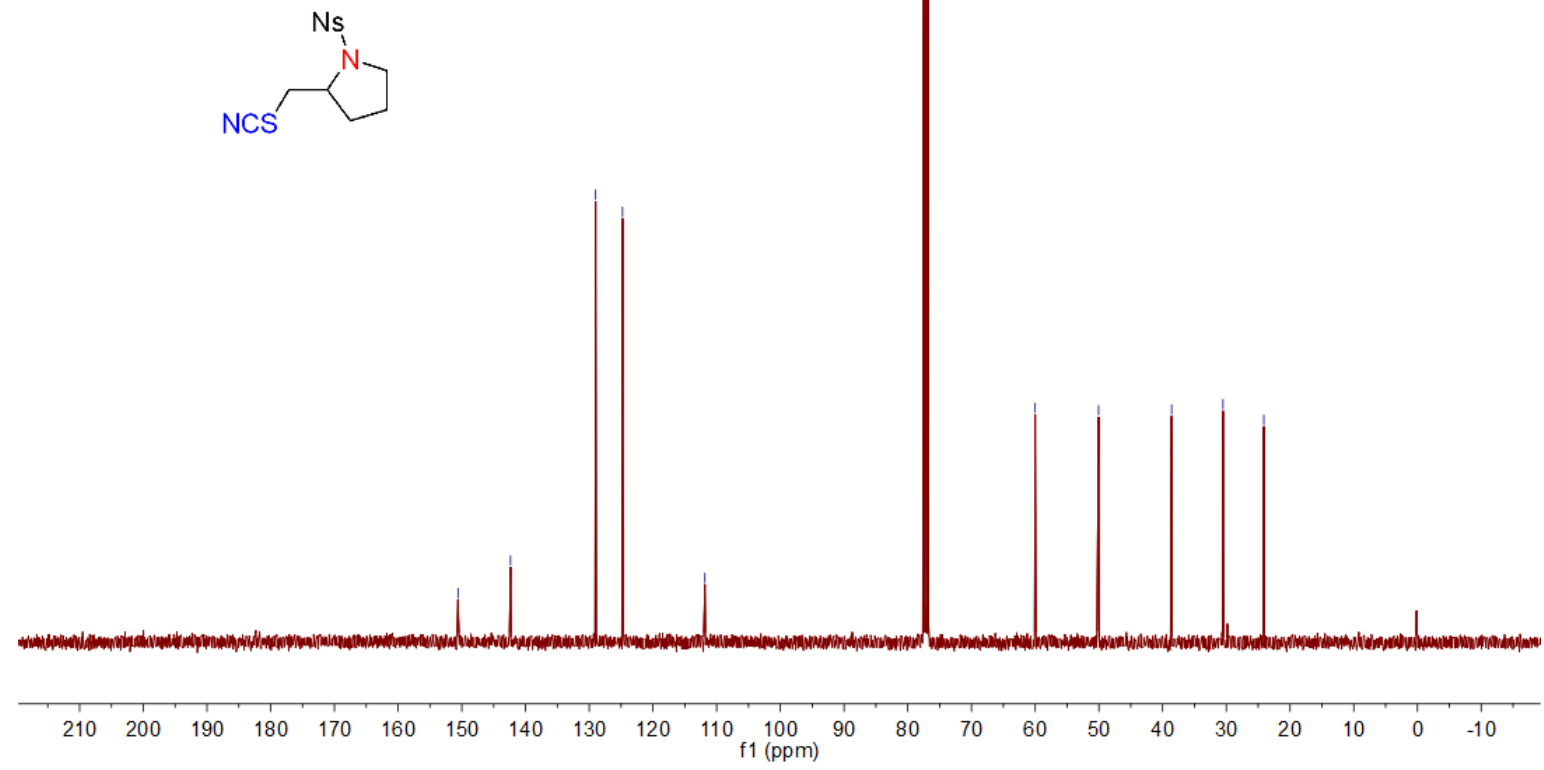

S43 
${ }^{1} \mathrm{H}$ NMR (400 $\left.\mathrm{MHz}, \mathrm{CDCl}_{3}\right)$ spectrum of 4,4-Dimethyl-1-((4-nitrophenyl)sulfonyl)-2-(thiocyanatomethyl) pyrrolidine (2j)
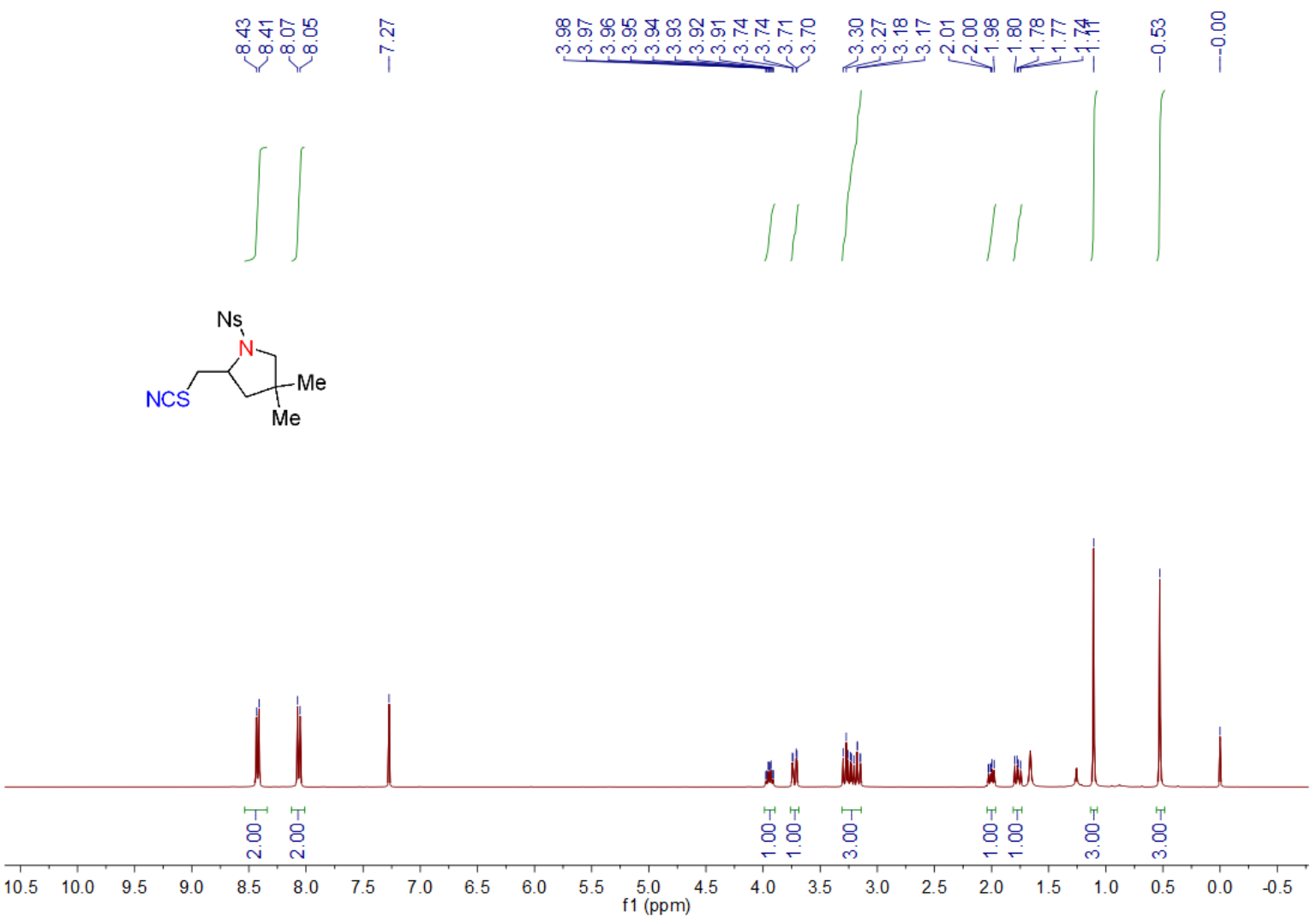

${ }^{13} \mathrm{C}$ NMR (101 $\left.\mathrm{MHz}, \mathrm{CDCl}_{3}\right)$ spectrum of 4,4-Dimethyl-1-((4-nitrophenyl)sulfonyl)-2-(thiocyanatomethyl) pyrrolidine (2j)

i

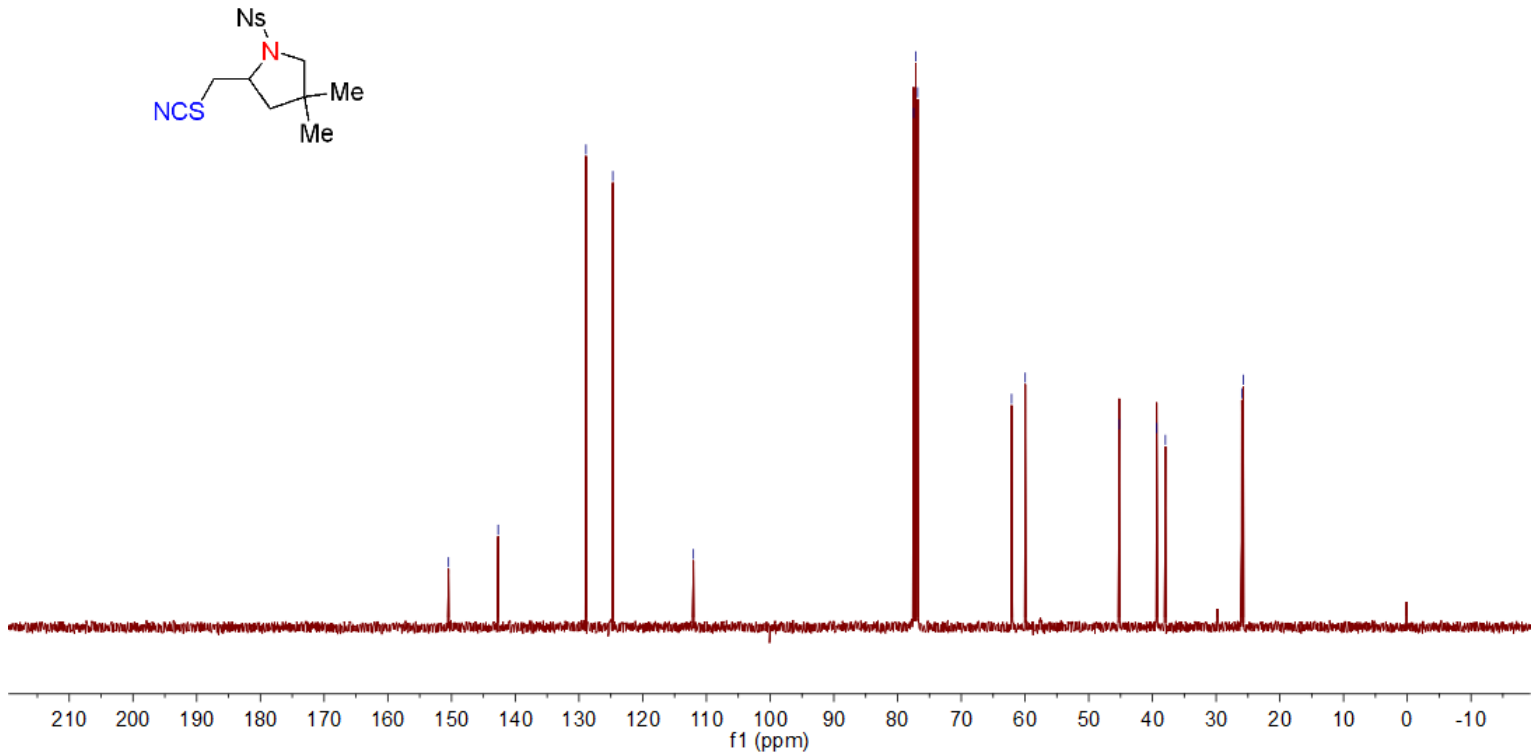


${ }^{1} \mathrm{H}$ NMR (400 MHz, $\mathrm{CDCl}_{3}$ ) spectrum of 4,4-Dimethyl-1-((4-nitrophenyl)sulfonyl)-2-(1-thiocyanatopropyl) pyrrolidine (2k)
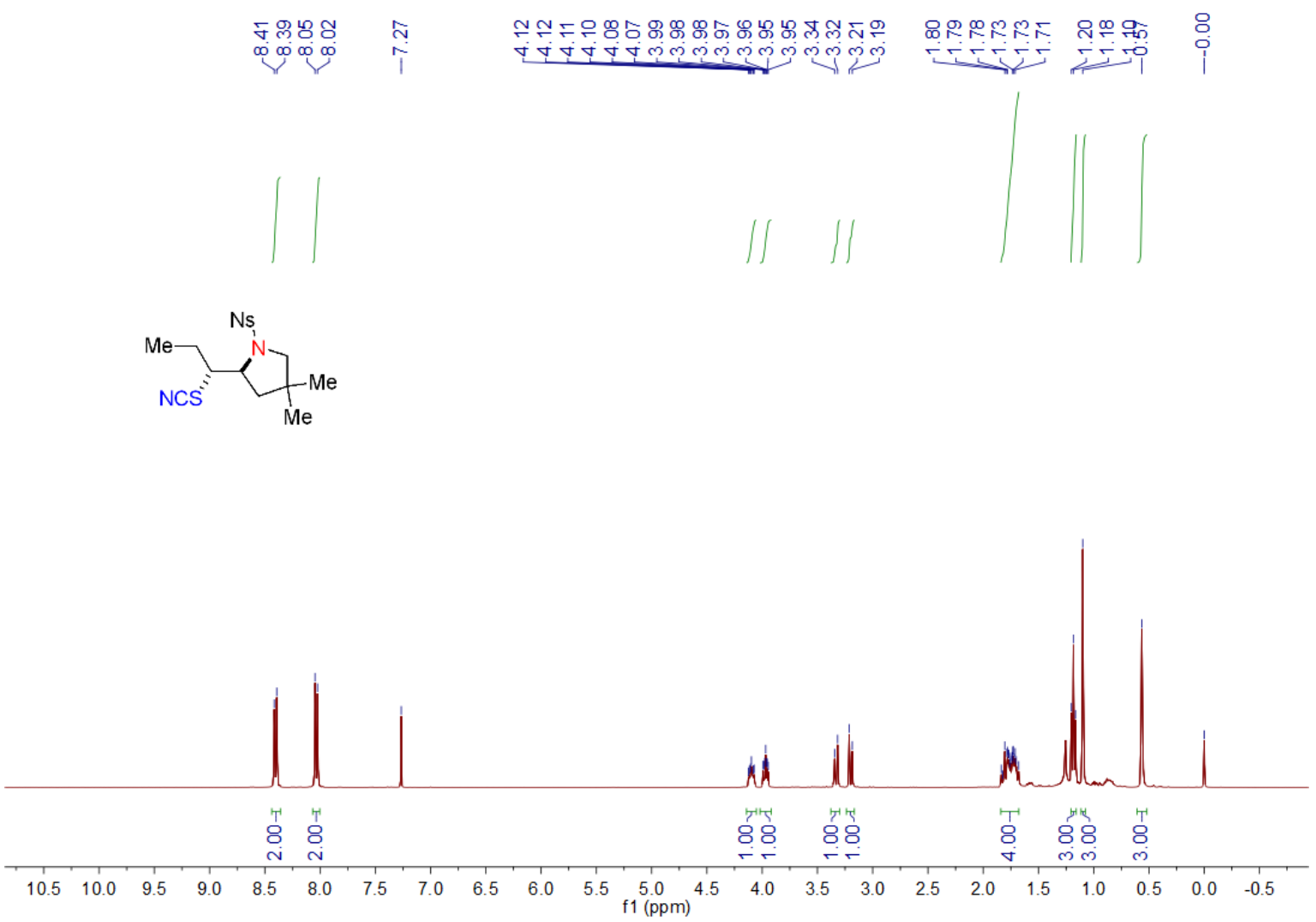

${ }^{13} \mathrm{C}$ NMR (101 MHz, $\left.\mathrm{CDCl}_{3}\right)$ spectrum of 4,4-Dimethyl-1-((4-nitrophenyl)sulfonyl)-2-(1-thiocyanatopropyl) pyrrolidine (2k)

穴
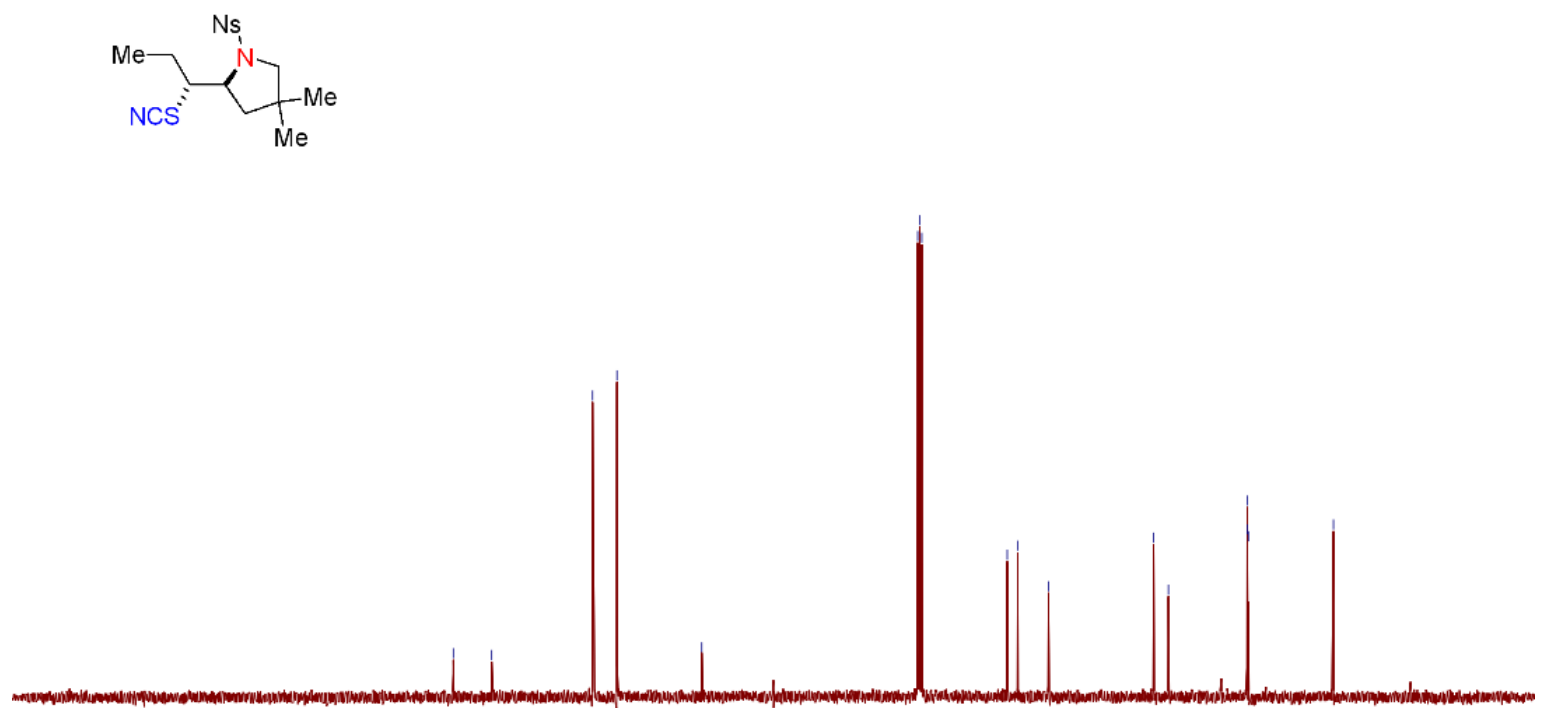

$\begin{array}{lllllllllllllllllllllll}210 & 200 & 190 & 180 & 170 & 160 & 150 & 140 & 130 & 120 & 110 & 100 & 90 & 80 & 70 & 60 & 50 & 40 & 30 & 20 & 10 & 0 & -10\end{array}$ 
${ }^{1} \mathrm{H}$ NMR (400 MHz, $\mathrm{CDCl}_{3}$ ) spectrum of 2-(1-Thiocyanatopentyl)-1-tosylpyrrolidine (2I)

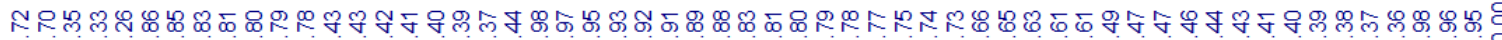

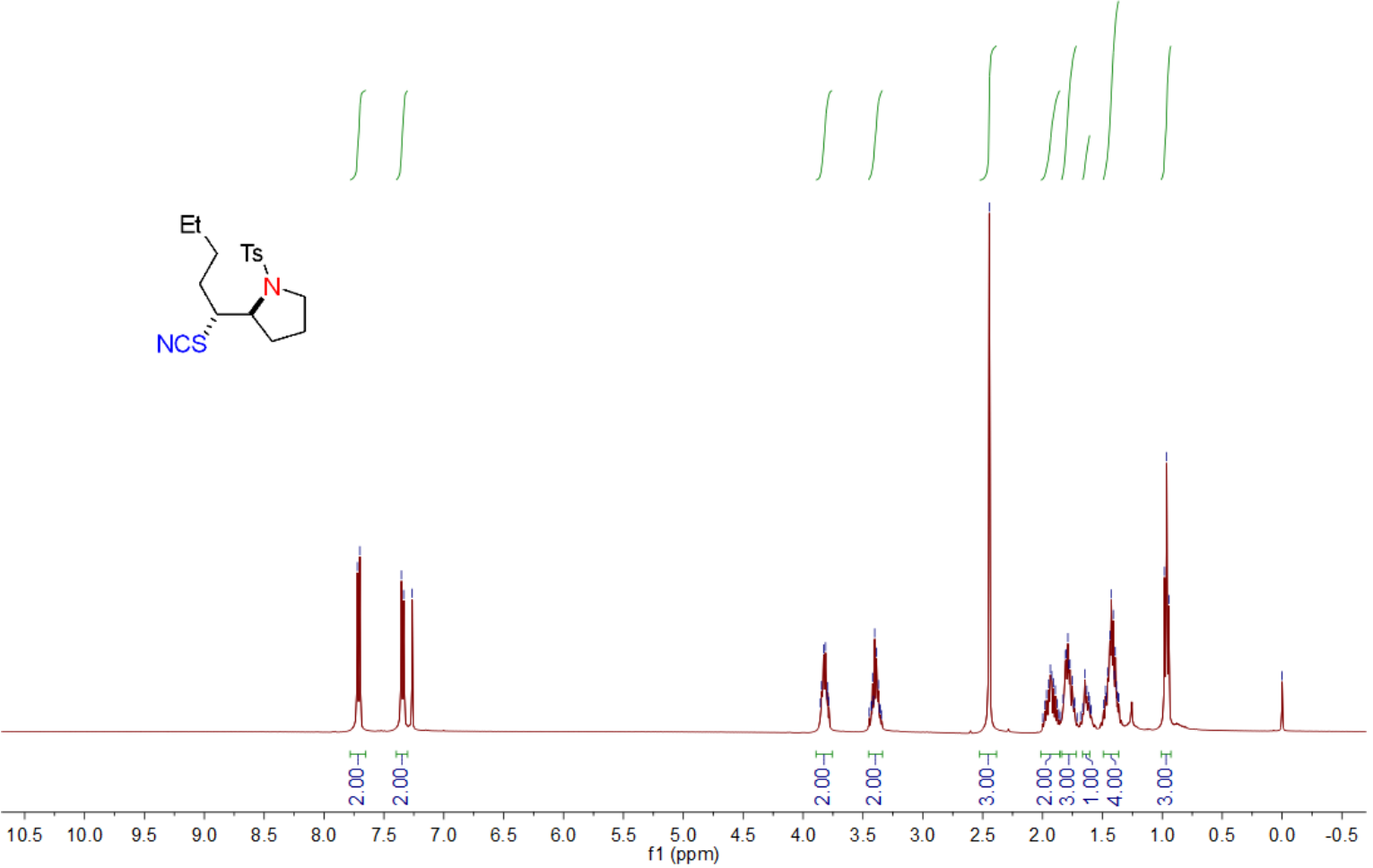

${ }^{13} \mathrm{C}$ NMR (101 MHz, $\mathrm{CDCl}_{3}$ ) spectrum of 2-(1-Thiocyanatopentyl)-1-tosylpyrrolidine (2l)

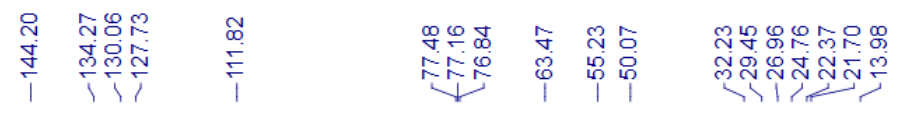

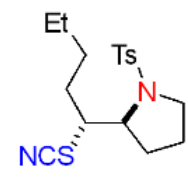

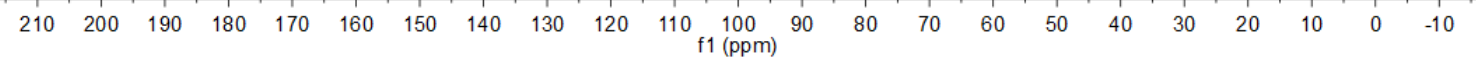


${ }^{1} \mathrm{H}$ NMR (400 MHz, $\mathrm{CDCl}_{3}$ ) spectrum of 1-(Methylsulfonyl)-2-(1-thiocyanatopentyl)pyrrolidine (2m)

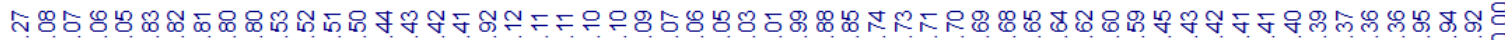

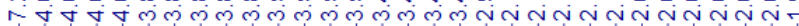

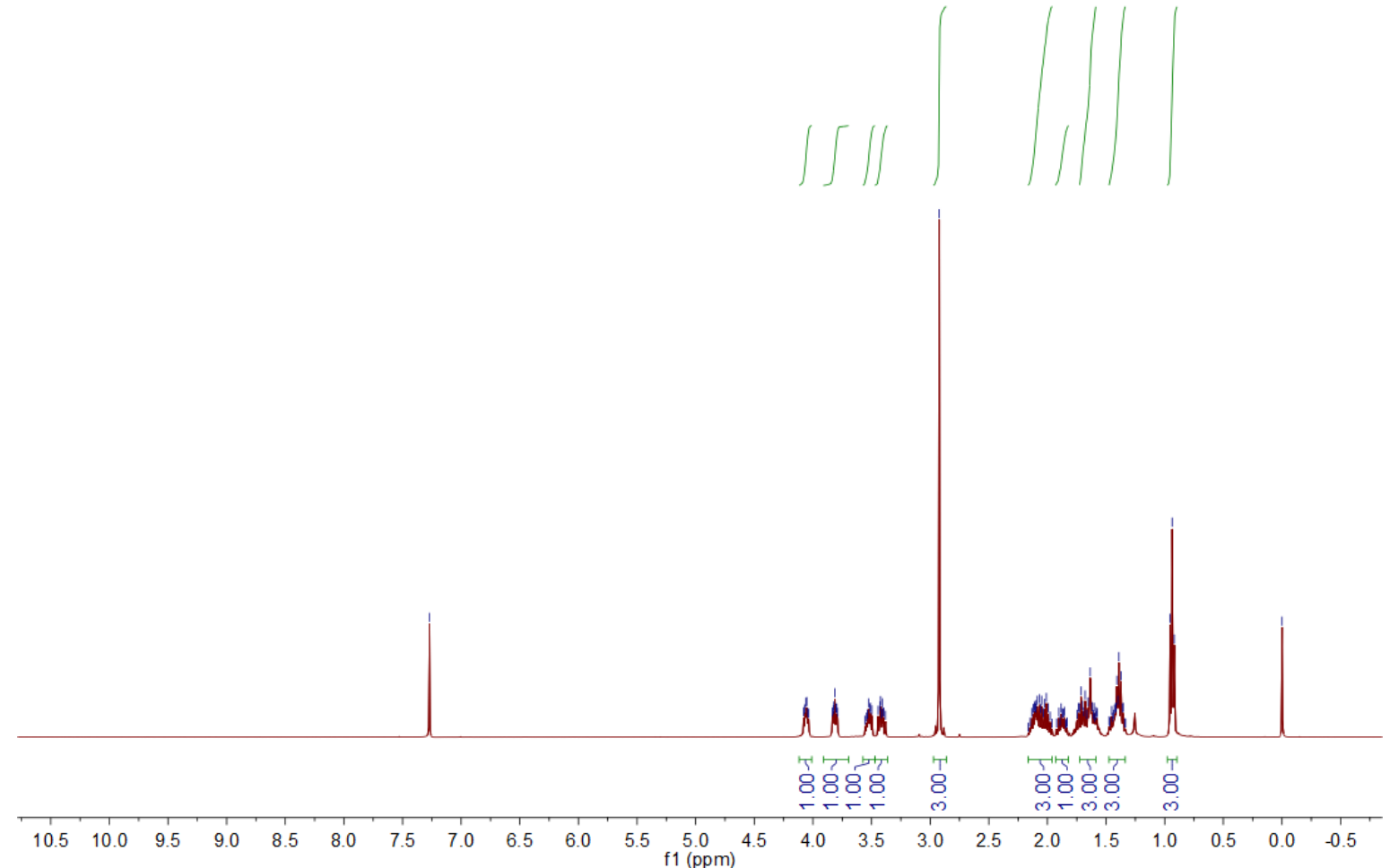

${ }^{13} \mathrm{C}$ NMR (101 MHz, $\mathrm{CDCl}_{3}$ ) spectrum of 1-(Methylsulfonyl)-2-(1-thiocyanatopentyl)pyrrolidine (2m)

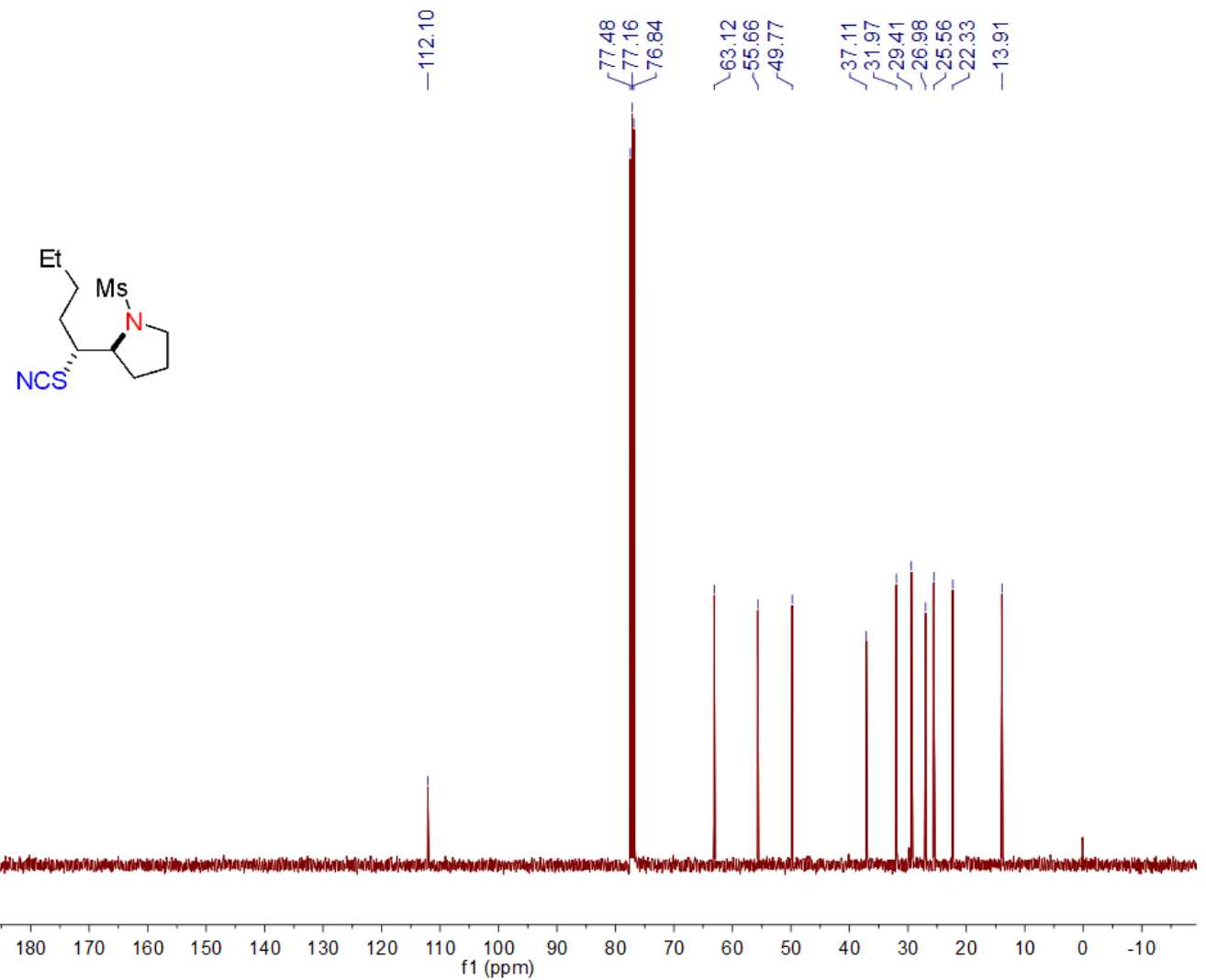


${ }^{1} \mathrm{H}$ NMR (400 MHz, $\mathrm{CDCl}_{3}$ ) spectrum of 1-((4-Nitrophenyl)sulfonyl)-2-phenyl-3-thiocyanatopiperidine (3n)

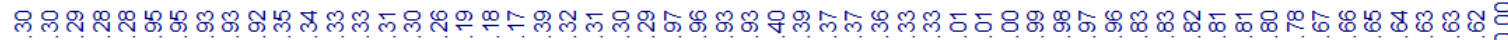

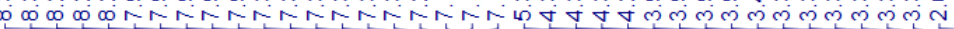
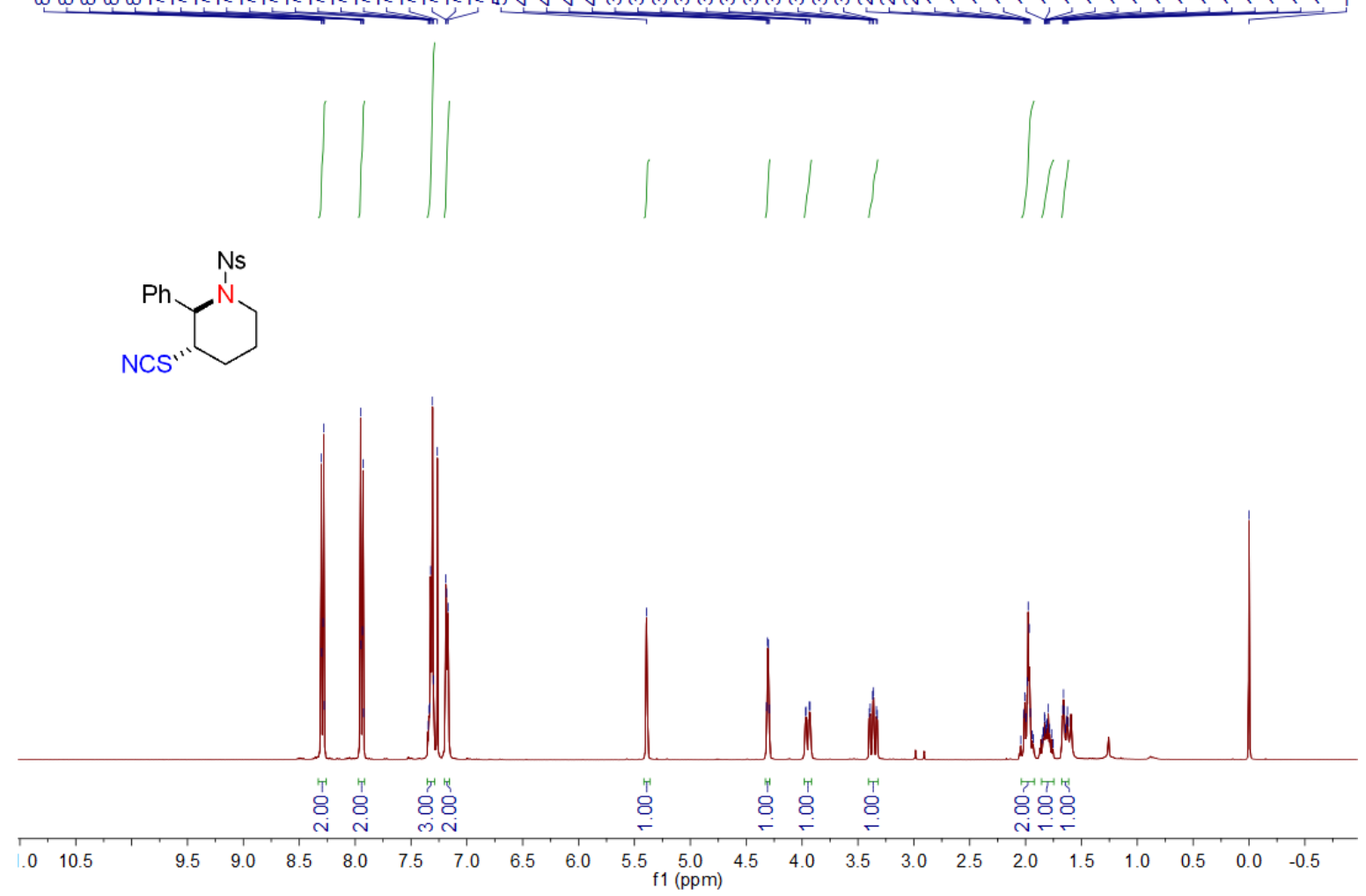

${ }^{13} \mathrm{C}$ NMR (101 MHz, $\mathrm{CDCl}_{3}$ ) spectrum of 1-((4-Nitrophenyl)sulfonyl)-2-phenyl-3-thiocyanatopiperidine (3n)

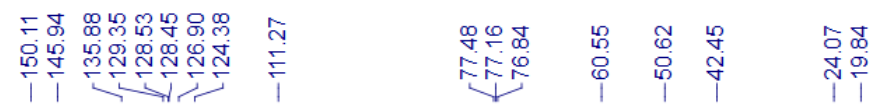

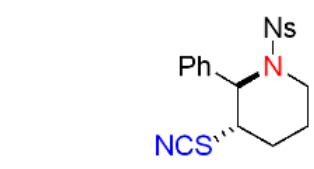

$\begin{array}{llllllllllllllllllllll}210 & 200 & 190 & 180 & 170 & 160 & 150 & 140 & 130 & 120 & 110 \underset{\mathrm{f} 1(\mathrm{ppm})}{100} & 90 & 80 & 70 & 60 & 50 & 40 & 30 & 20 & 10 & 0 & -10\end{array}$ 
${ }^{1} \mathrm{H}$ NMR (400 MHz, $\mathrm{CDCl}_{3}$ ) spectrum of 1-((4-Nitrophenyl)sulfonyl)-3-thiocyanato-2-(p-tolyl) piperidine (3o)

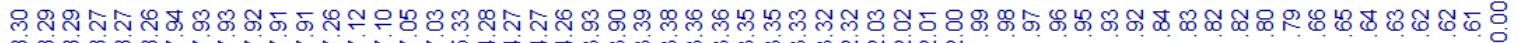

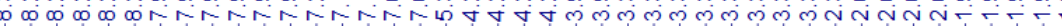

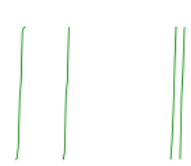<smiles>Cc1ccc(C2[C@H](C#N)CCCN2N)cc1</smiles>

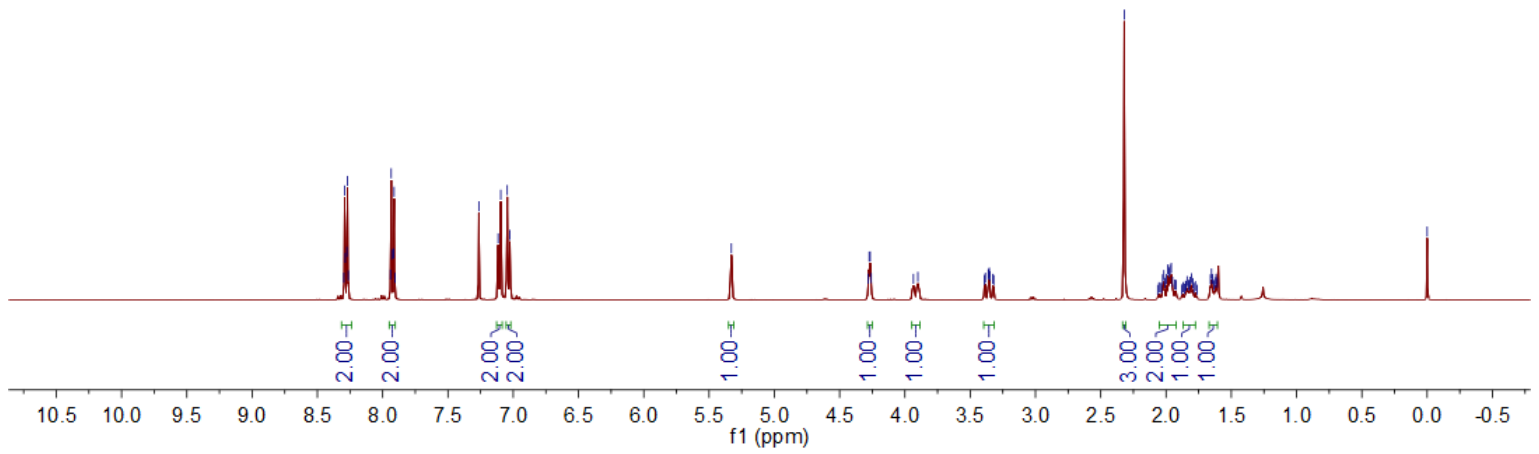

${ }^{13} \mathrm{C}$ NMR (101 MHz, $\mathrm{CDCl}_{3}$ ) spectrum of 1-((4-Nitrophenyl)sulfonyl)-3-thiocyanato-2-(p-tolyl) piperidine (3o)

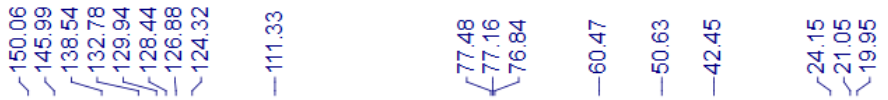<smiles>Cc1ccc(C2[C@H]([Se])CCCN2[N+]#N)cc1</smiles>

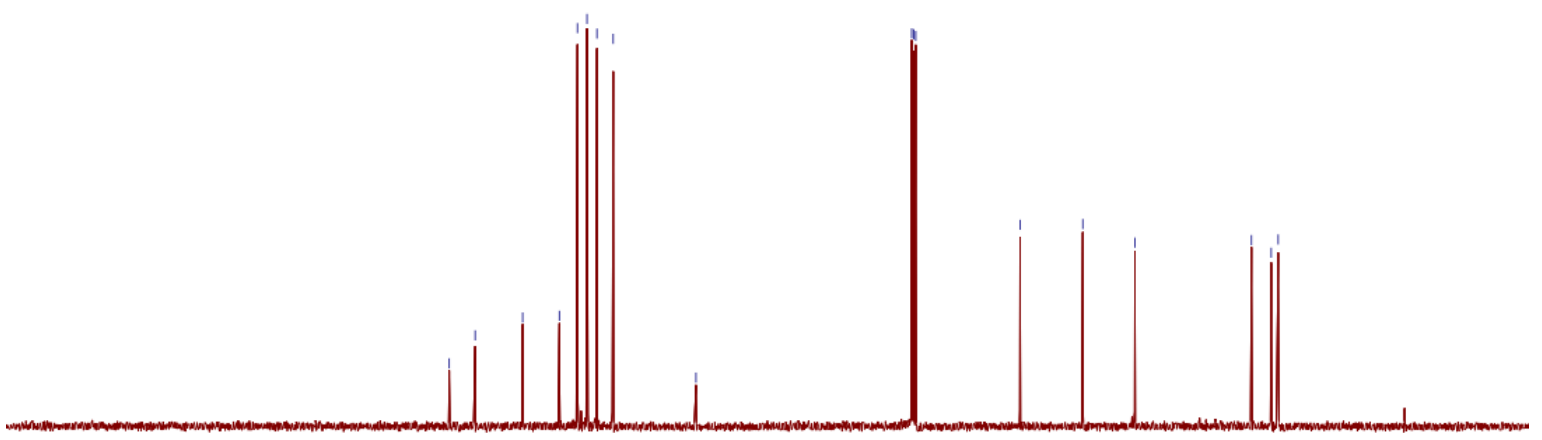

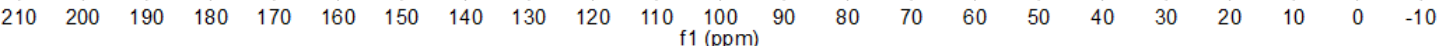


${ }^{1} \mathrm{H} \quad \mathrm{NMR} \quad\left(400 \quad \mathrm{MHz}, \quad \mathrm{CDCl}_{3}\right) \quad$ spectrum $\quad$ of $\quad$ 2-(4-Chlorophenyl)-1-((4-nitrophenyl)sulfonyl)-3thiocyanatopiperidine (3p)

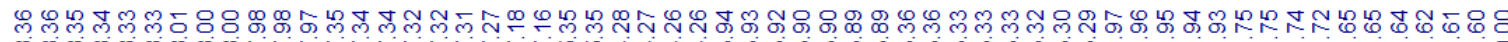

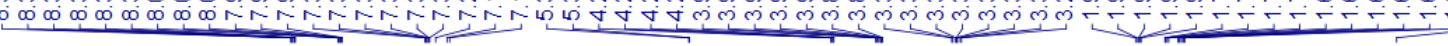

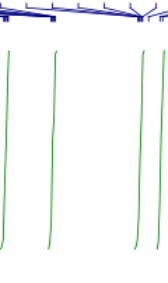<smiles>N#C[C@@H]1CCCN([N+](=O)[O-])C1c1ccc(Cl)cc1</smiles>

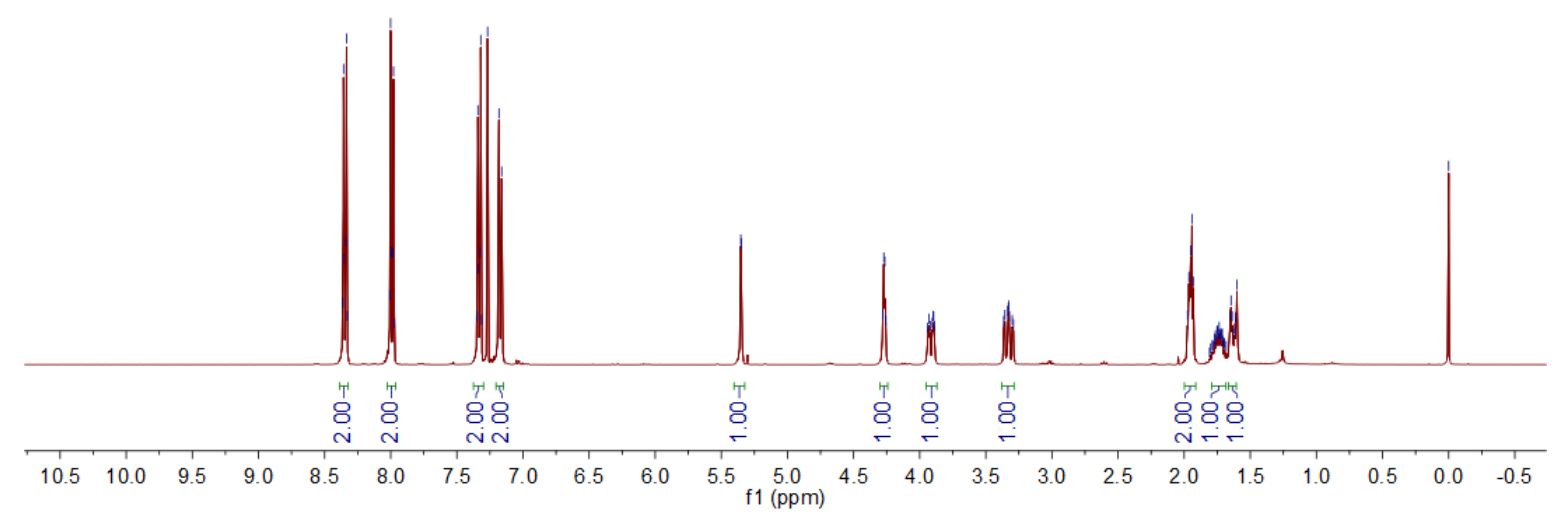

${ }^{13} \mathrm{C} \quad \mathrm{NMR} \quad\left(101 \quad \mathrm{MHz}, \quad \mathrm{CDCl}_{3}\right) \quad$ spectrum of 2-(4-Chlorophenyl)-1-((4-nitrophenyl)sulfonyl)-3thiocyanatopiperidine (3p)

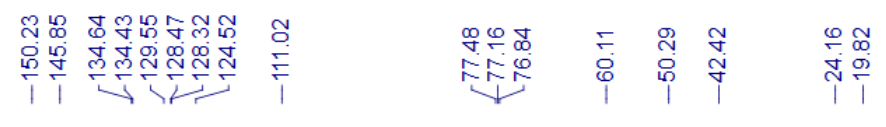

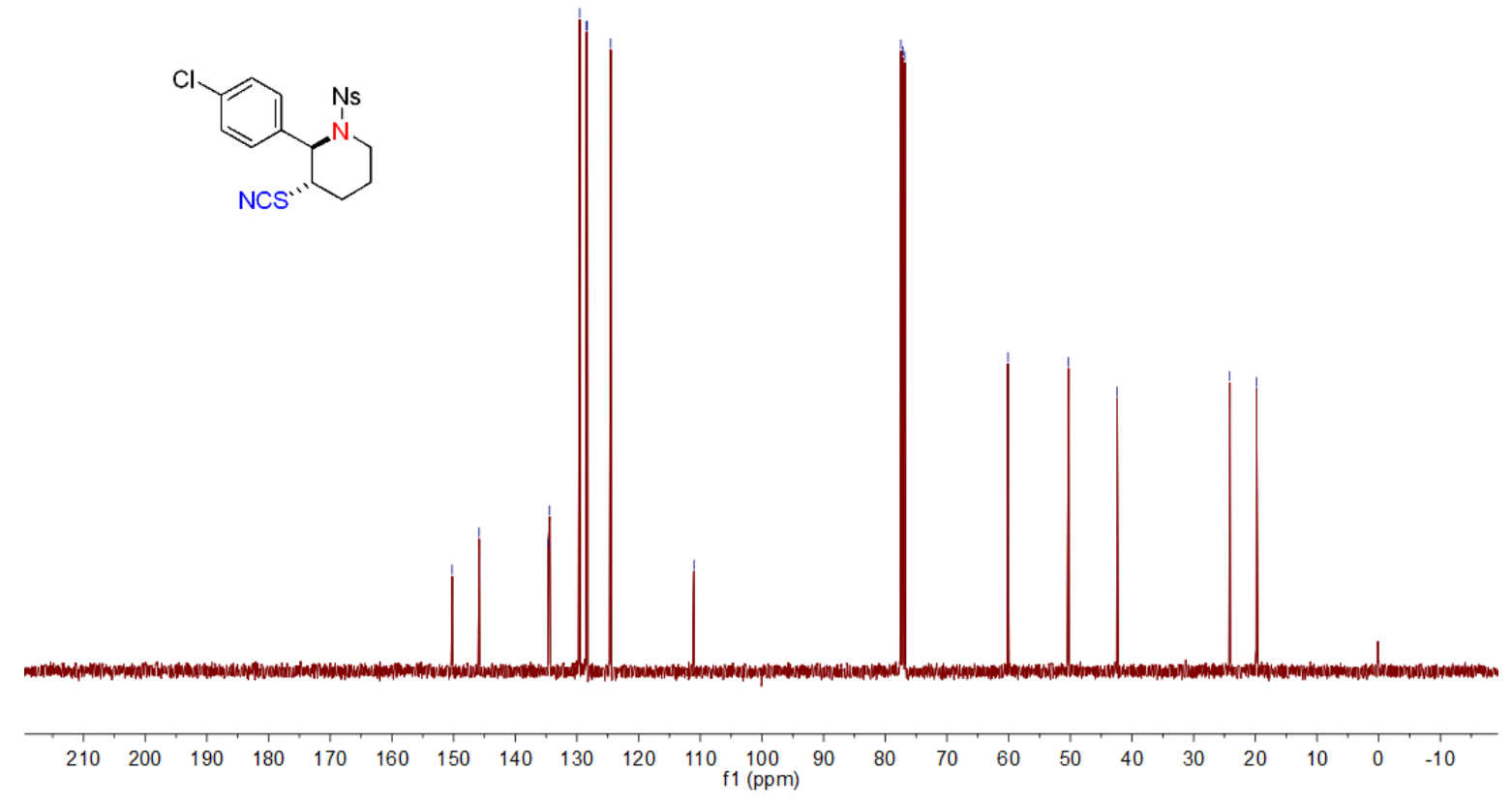


${ }^{1} \mathrm{H} \quad \mathrm{NMR} \quad\left(400 \quad \mathrm{MHz}, \quad \mathrm{CDCl}_{3}\right) \quad$ spectrum of 2-(4-Methoxyphenyl)-1-((4-nitrophenyl)sulfonyl)-3thiocyanatopiperidine (3q)

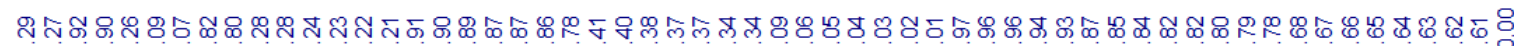

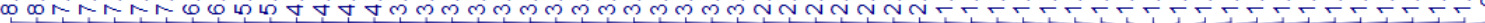

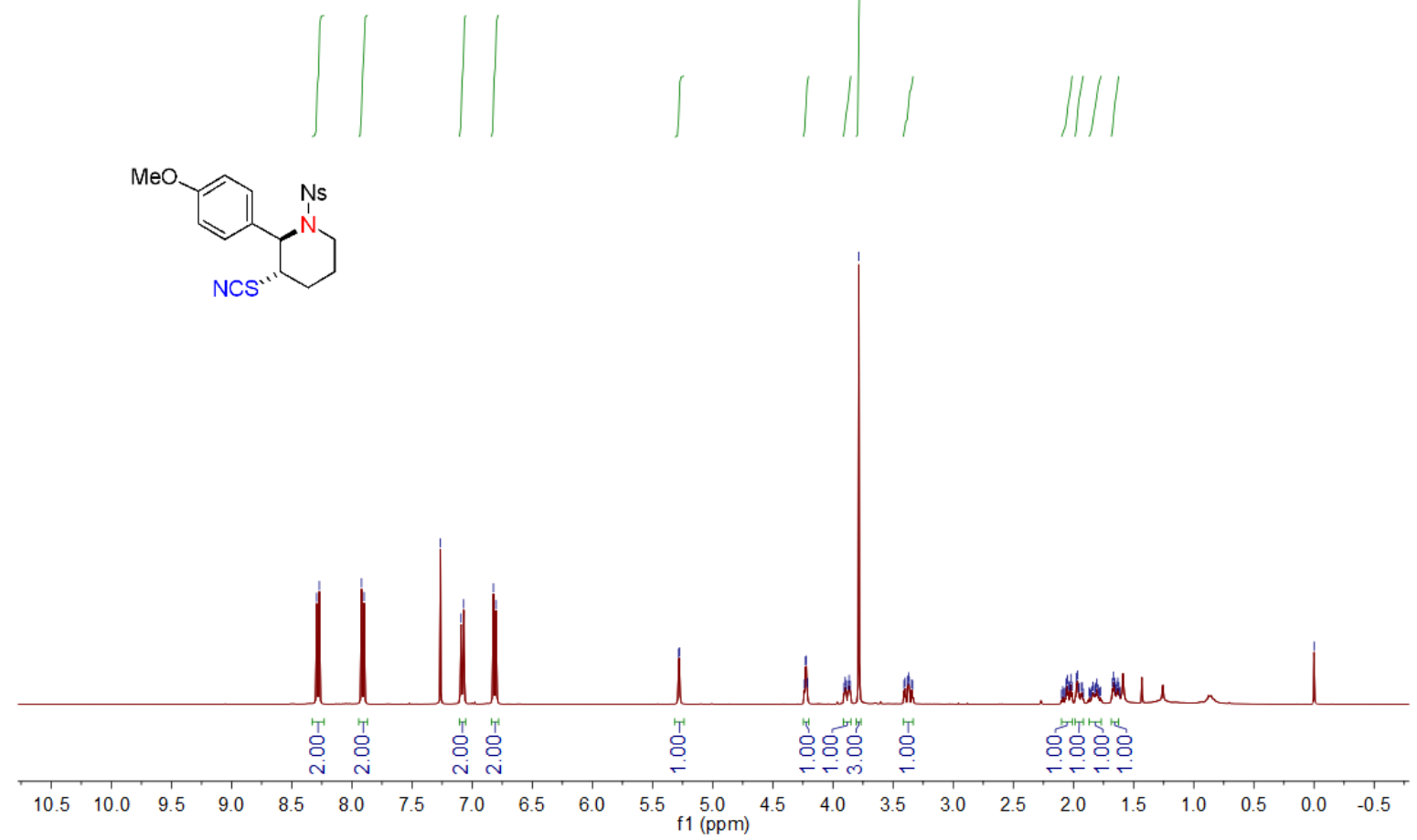

${ }^{13} \mathrm{C} \quad \mathrm{NMR} \quad\left(101 \quad \mathrm{MHz}, \quad \mathrm{CDCl}_{3}\right) \quad$ spectrum of 2-(4-Methoxyphenyl)-1-((4-nitrophenyl)sulfonyl)-3thiocyanatopiperidine (3q)<smiles>COc1ccc(C2[C@H](C#N)CCCN2S(C)(=O)=O)cc1</smiles>

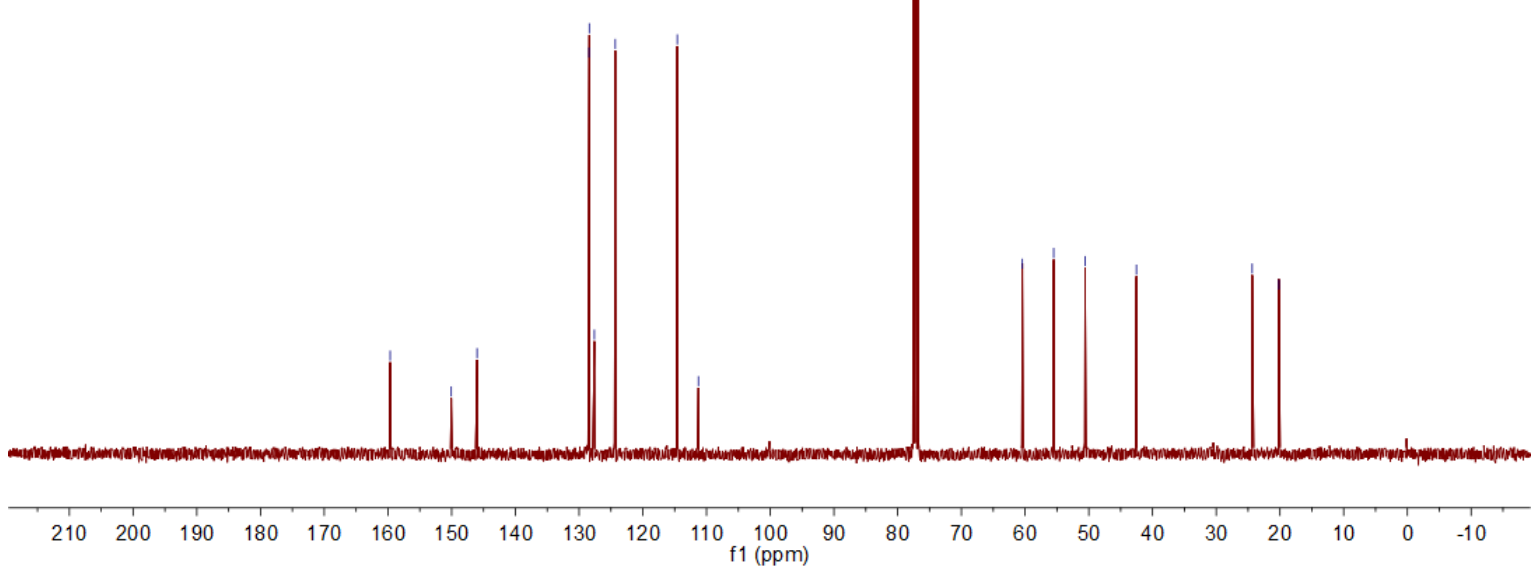




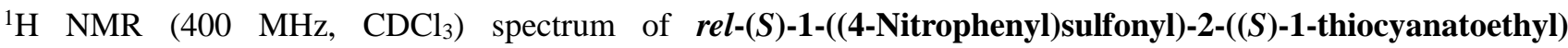
pyrrolidine (2r)

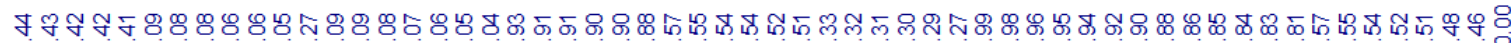
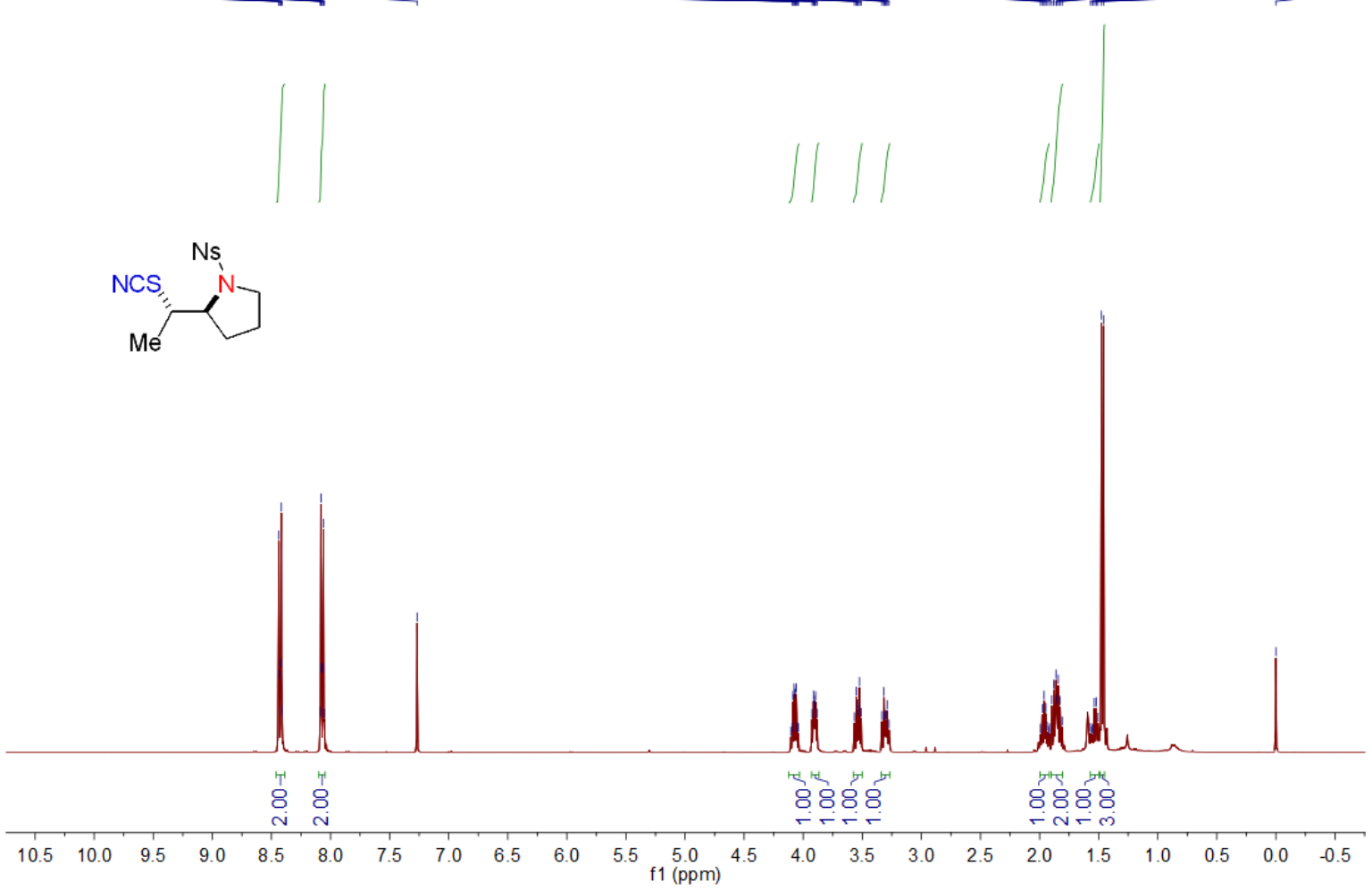

${ }^{13} \mathrm{C} \quad \mathrm{NMR}\left(101 \mathrm{MHz}, \mathrm{CDCl}_{3}\right)$ spectrum of rel-(S)-1-((4-Nitrophenyl)sulfonyl)-2-((S)-1-thiocyanatoethyl) pyrrolidine (2r)

\begin{tabular}{|c|c|c|c|c|c|c|}
\hline 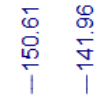 & 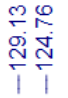 & 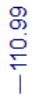 & 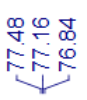 & $\begin{array}{l}0 \\
0 \\
01 \\
0 \\
1\end{array}$ & $\begin{array}{l}95 \\
0 \% \\
00 \\
11\end{array}$ & 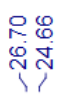 \\
\hline
\end{tabular}

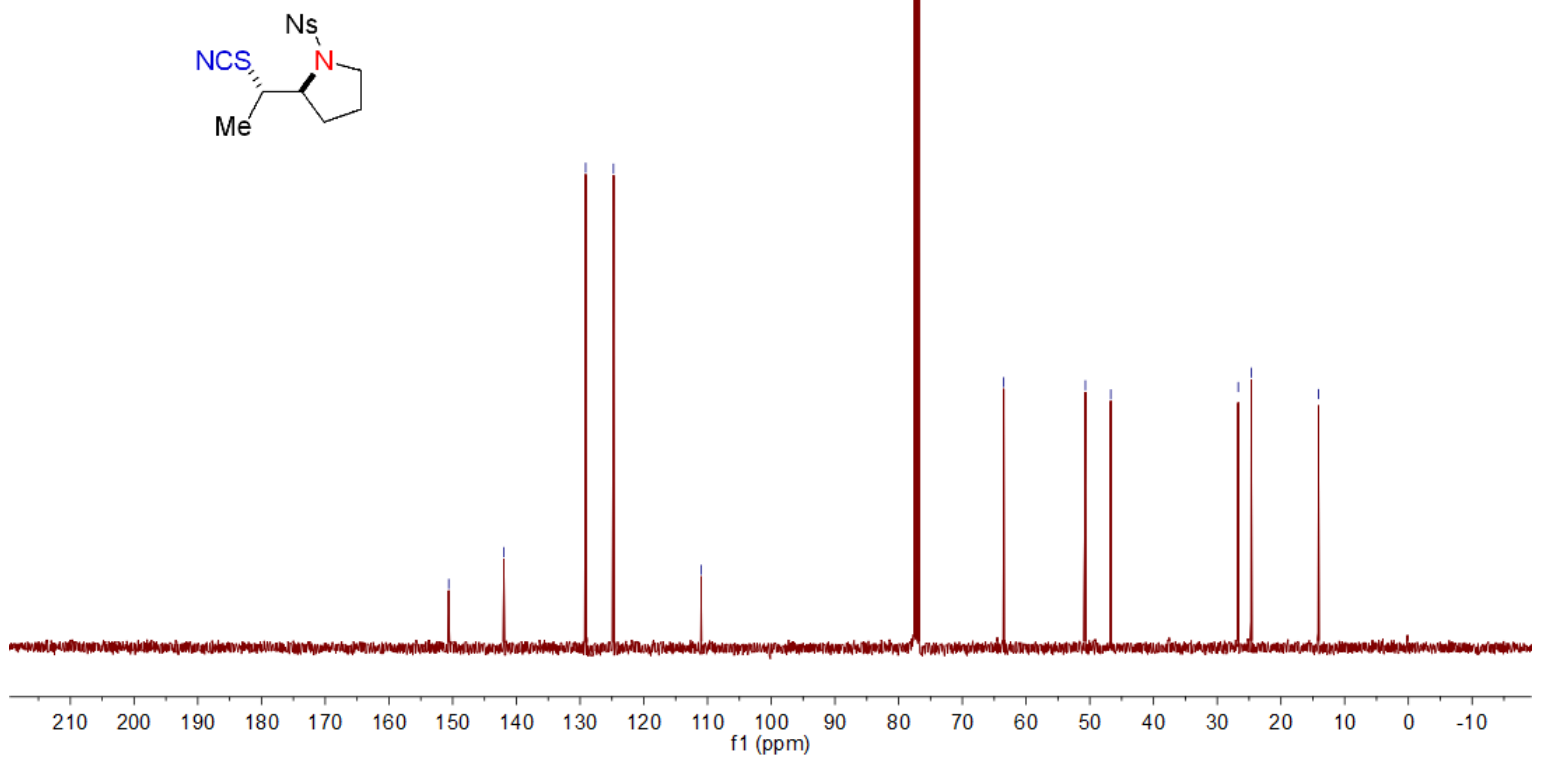


${ }^{1} \mathrm{H} \quad \mathrm{NMR} \quad\left(400 \mathrm{MHz}, \mathrm{CDCl}_{3}\right)$ spectrum of 1-((4-Nitrophenyl)sulfonyl)-2-phenyl-2-(1-thiocyanatohexyl) pyrrolidine (2s)

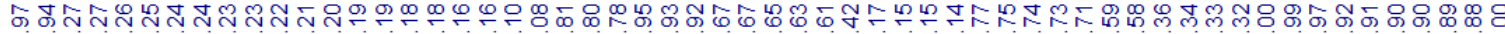

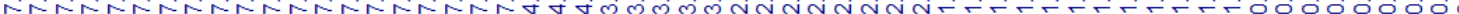

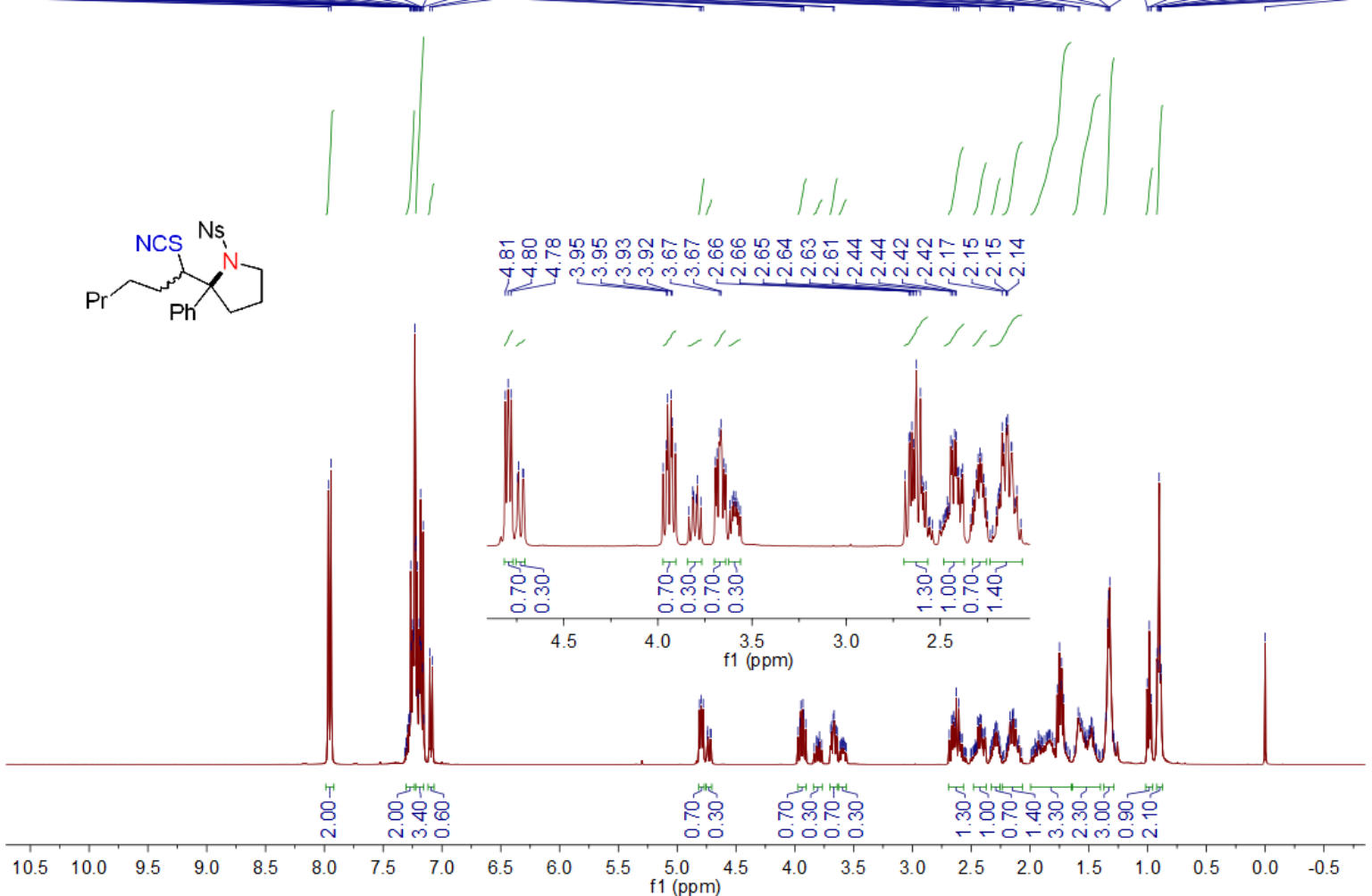

${ }^{13} \mathrm{C} \quad \mathrm{NMR} \quad\left(101 \mathrm{MHz}, \mathrm{CDCl}_{3}\right)$ spectrum of 1-((4-Nitrophenyl)sulfonyl)-2-phenyl-2-(1-thiocyanatohexyl) pyrrolidine (2s)

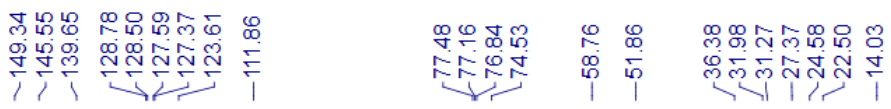

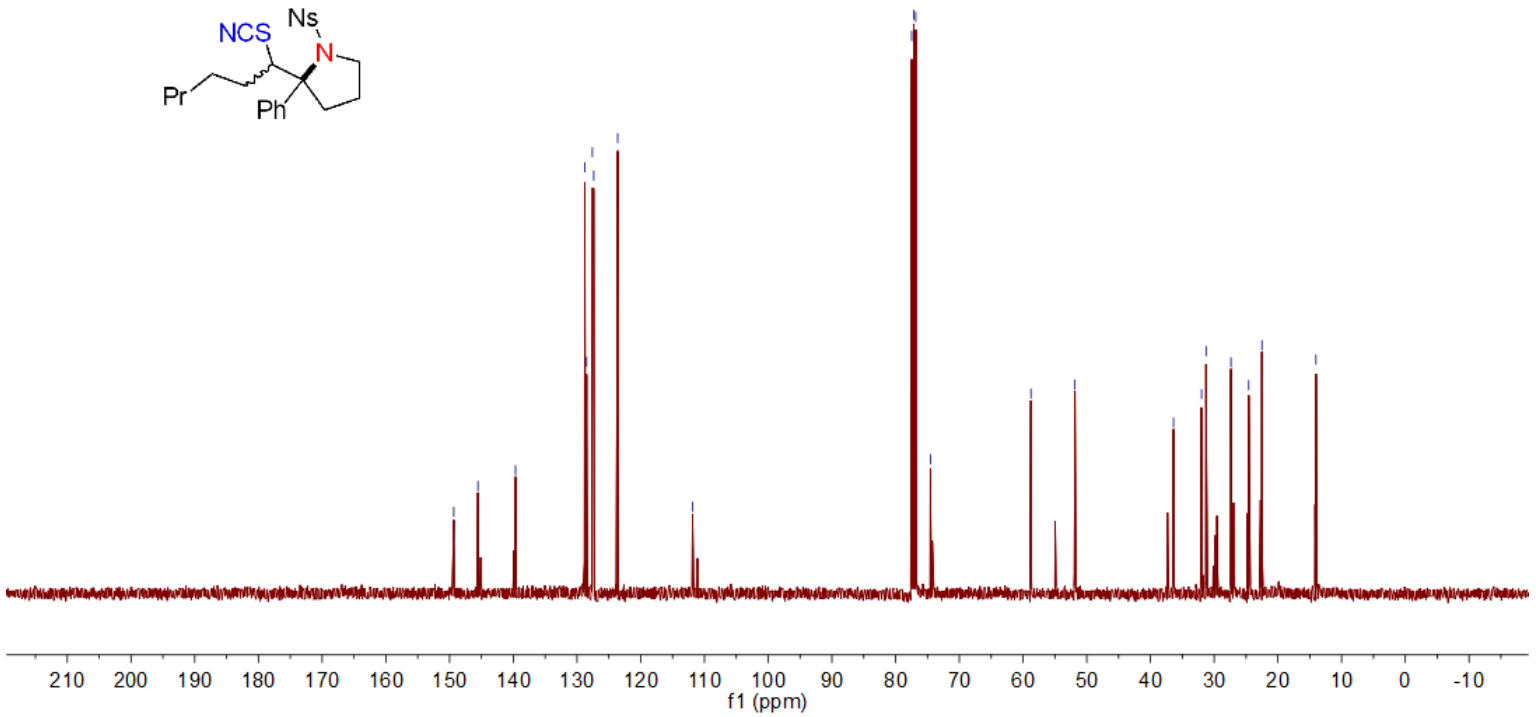


${ }^{1} \mathrm{H}$ NMR (400 MHz, $\left.\mathrm{CDCl}_{3}\right)$ spectrum of 2-(1-Thiocyanatopentyl)tetrahydrofuran (2t) + 2-Butyl-3-thiocyanatotetrahydro-2H-pyran $(3 \mathrm{t})$

సุ.
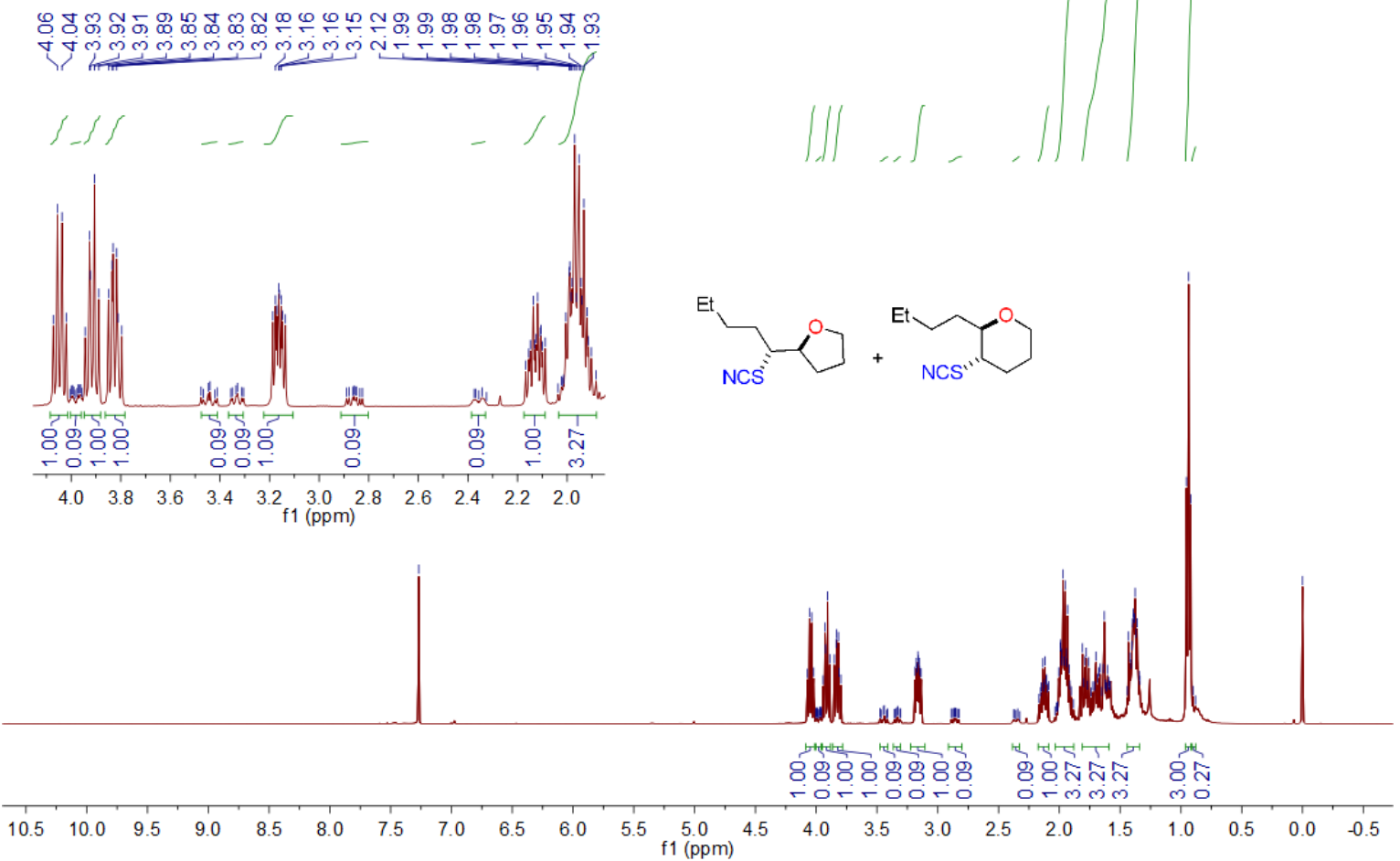

${ }^{13} \mathrm{C}$ NMR $\left(101 \mathrm{MHz}, \mathrm{CDCl}_{3}\right)$ spectrum of 2-(1-Thiocyanatopentyl)tetrahydrofuran (2t) + 2-Butyl-3-thiocyanatotetrahydro-2H-pyran $(3 \mathrm{t})$

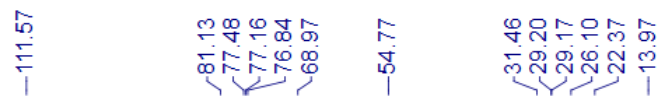

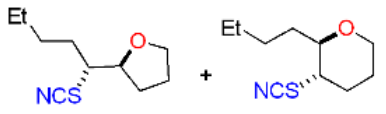


${ }^{1} \mathrm{H}$ NMR (400 MHz, $\mathrm{CDCl}_{3}$ ) spectrum of 2-Phenyl-3-thiocyanatotetrahydrofuran (2u)

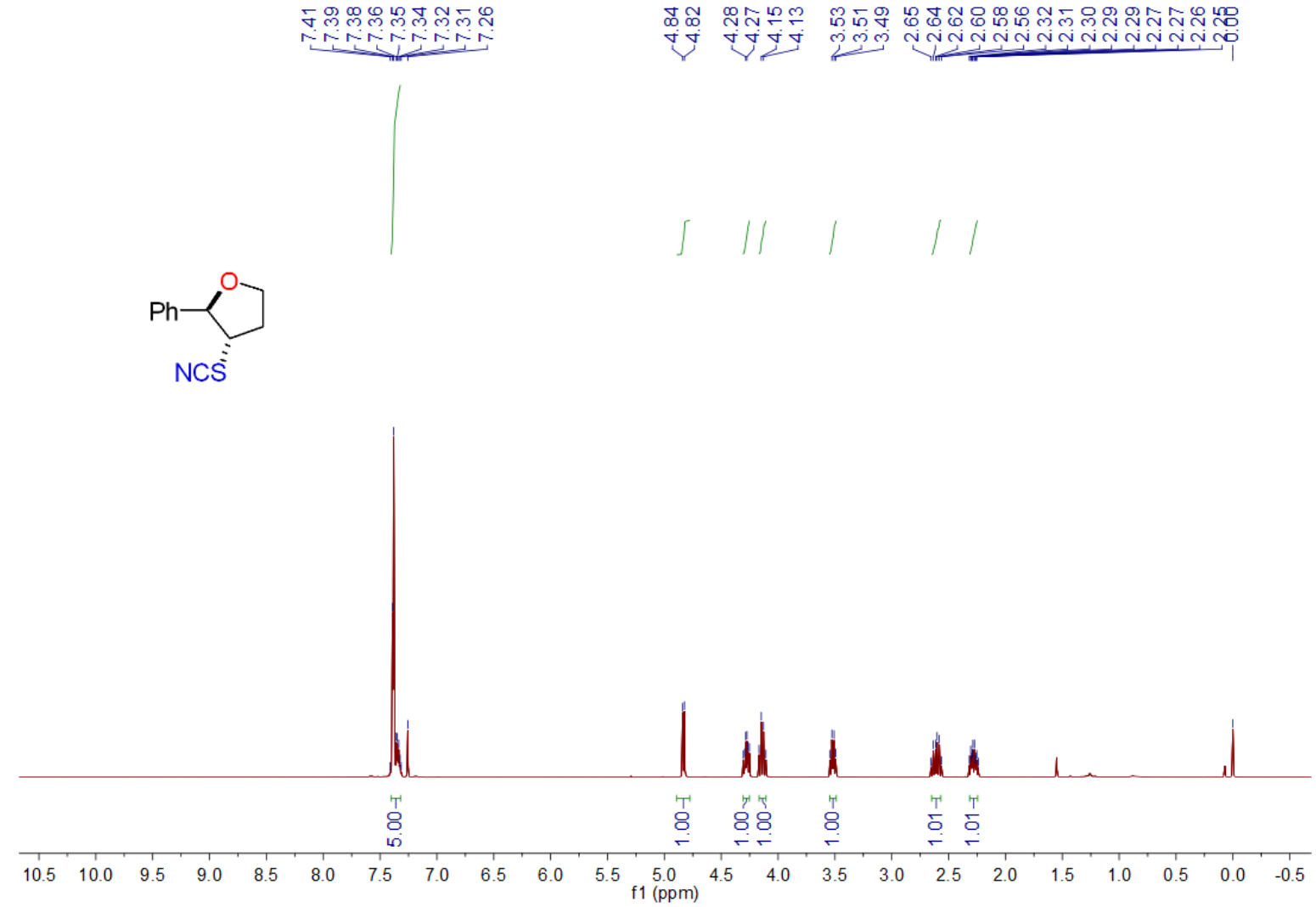

${ }^{13} \mathrm{C}$ NMR (101 MHz, $\mathrm{CDCl}_{3}$ ) spectrum of 2-Phenyl-3-thiocyanatotetrahydrofuran (2u)

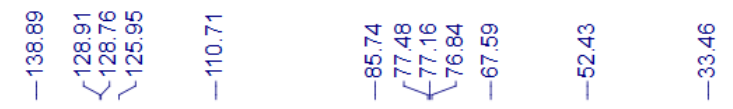

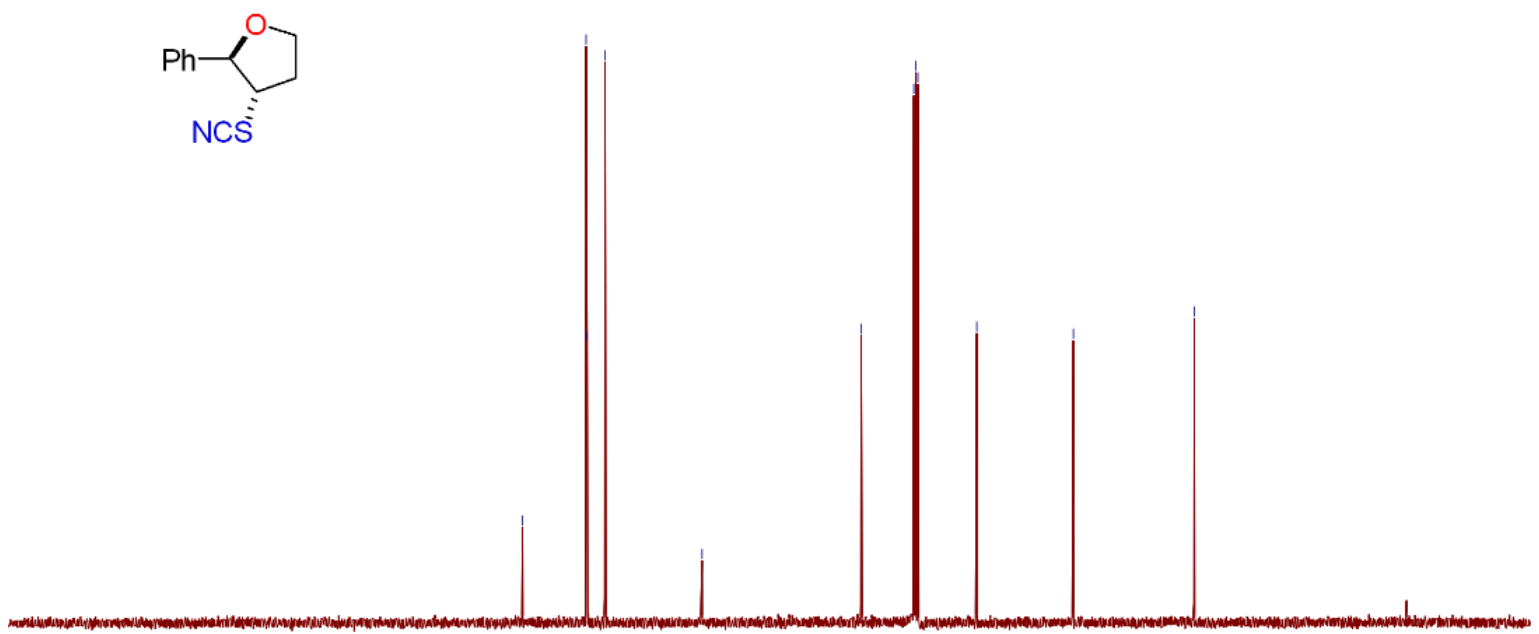

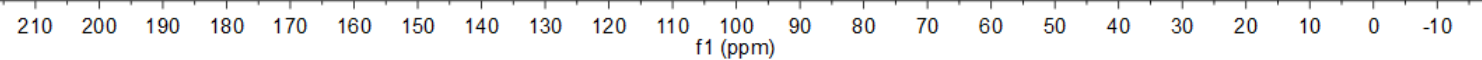


${ }^{1} \mathrm{H}$ NMR (400 MHz, $\mathrm{CDCl}_{3}$ ) spectrum of 2-Phenyl-3-thiocyanatotetrahydro-2H-pyran (2v)

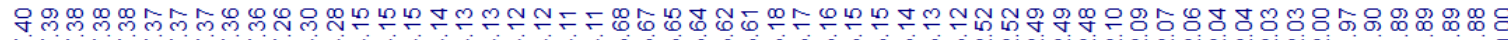

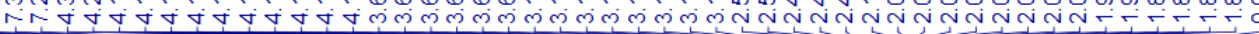

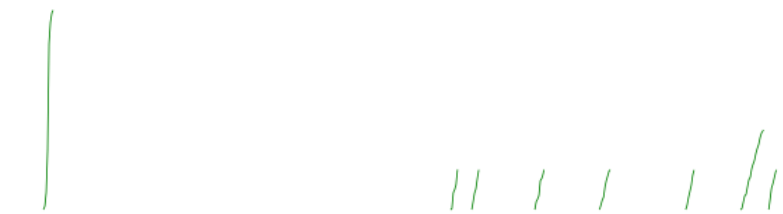<smiles>N#[N+][C@H]1CCCOC1c1ccccc1</smiles>

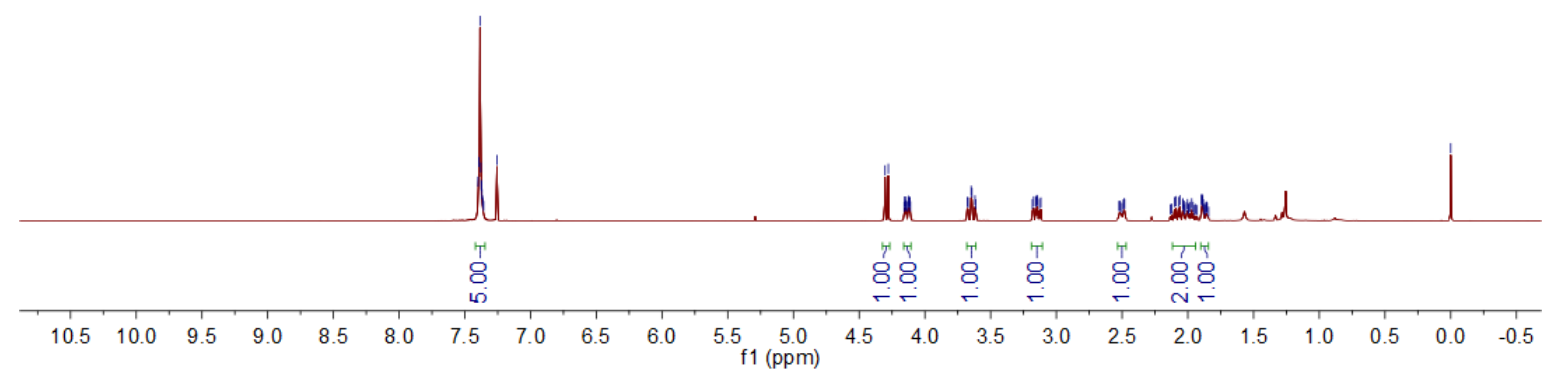

${ }^{13} \mathrm{C}$ NMR (101 MHz, $\mathrm{CDCl}_{3}$ ) spectrum of 2-Phenyl-3-thiocyanatotetrahydro-2H-pyran (2v)

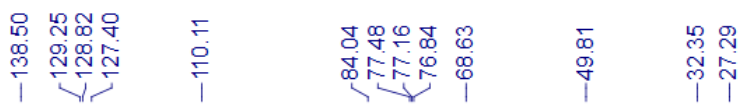<smiles>N#[N+][C@H]1CCCOC1c1ccccc1</smiles>

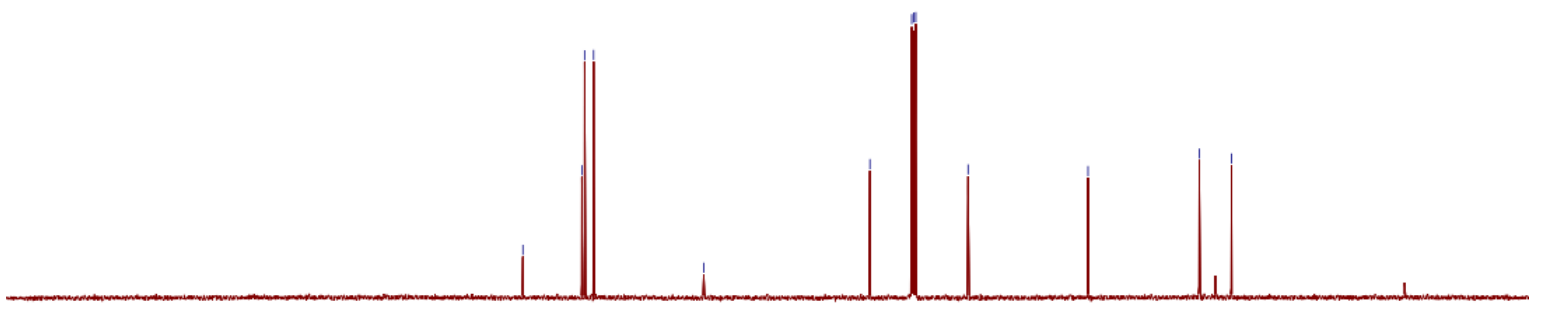

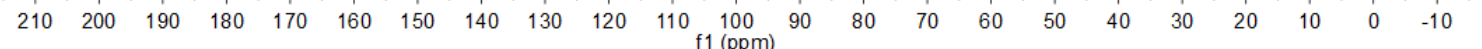


${ }^{1} \mathrm{H}$ NMR (400 MHz, $\mathrm{CDCl}_{3}$ ) spectrum of 2-Phenyl-2-(thiocyanatomethyl)tetrahydrofuran (2w)

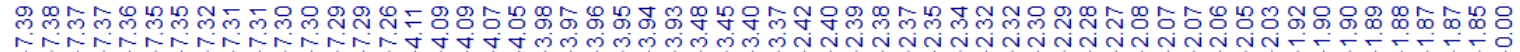
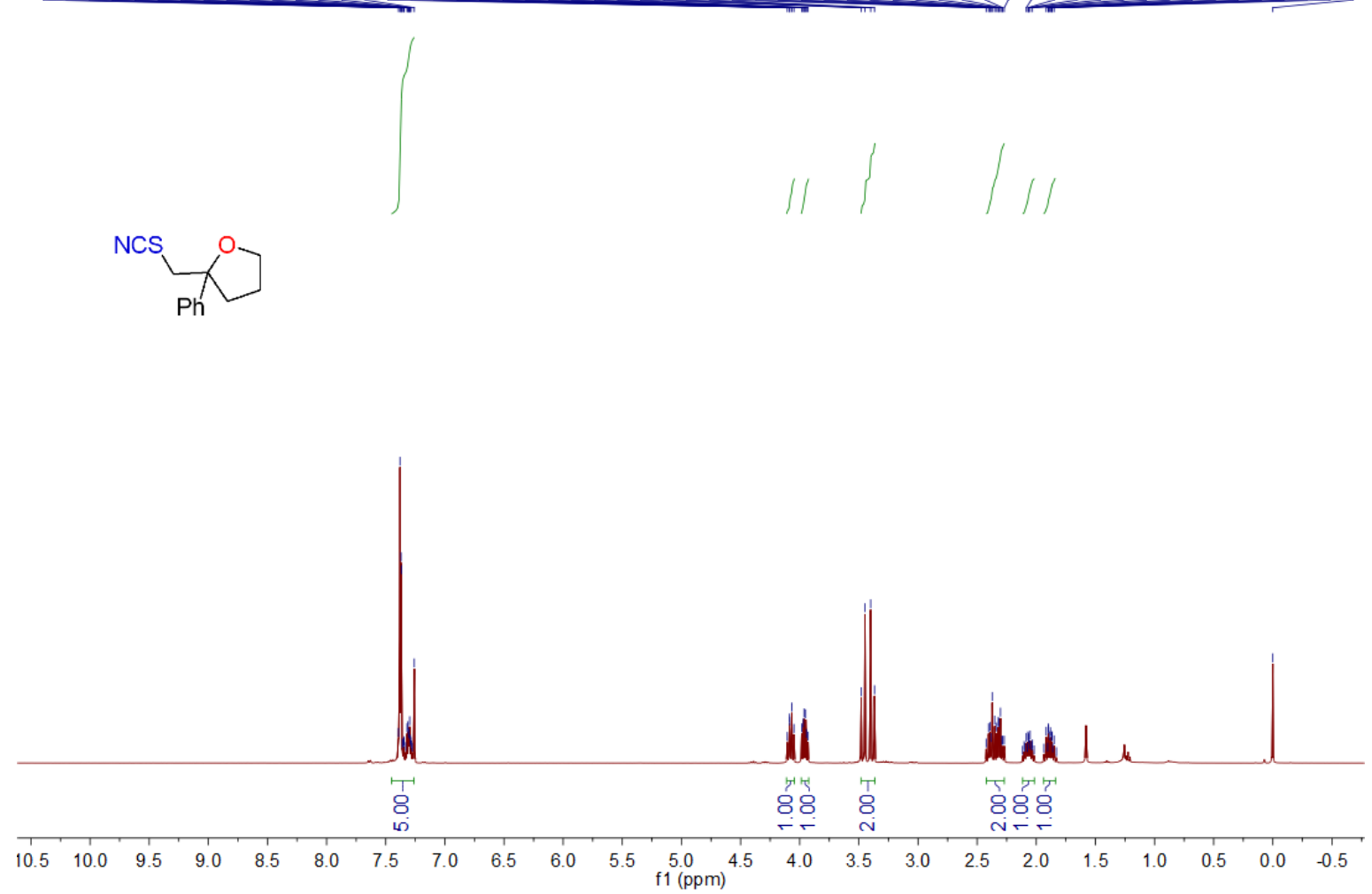

${ }^{13} \mathrm{C}$ NMR (101 MHz, $\mathrm{CDCl}_{3}$ ) spectrum of 2-Phenyl-2-(thiocyanatomethyl)tetrahydrofuran (2w)

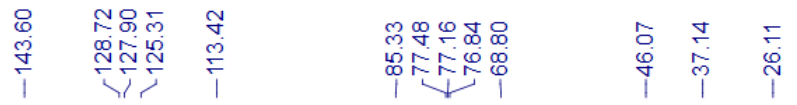

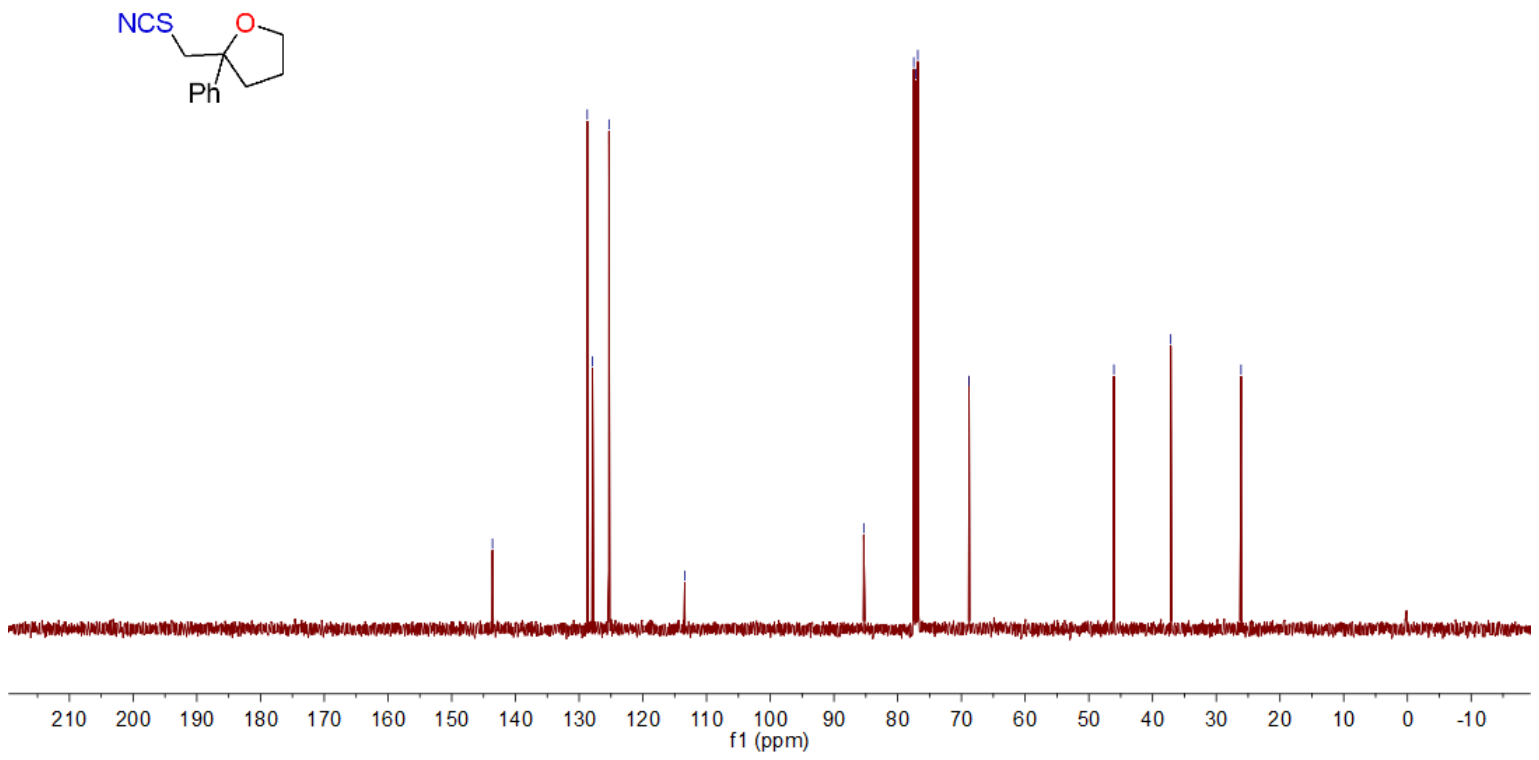


${ }^{1} \mathrm{H}$ NMR $\left(400 \mathrm{MHz}, \mathrm{CDCl}_{3}\right)$ spectrum of 5-Phenyl-5-(thiocyanatomethyl)dihydrofuran-2(3H)-one (2x)
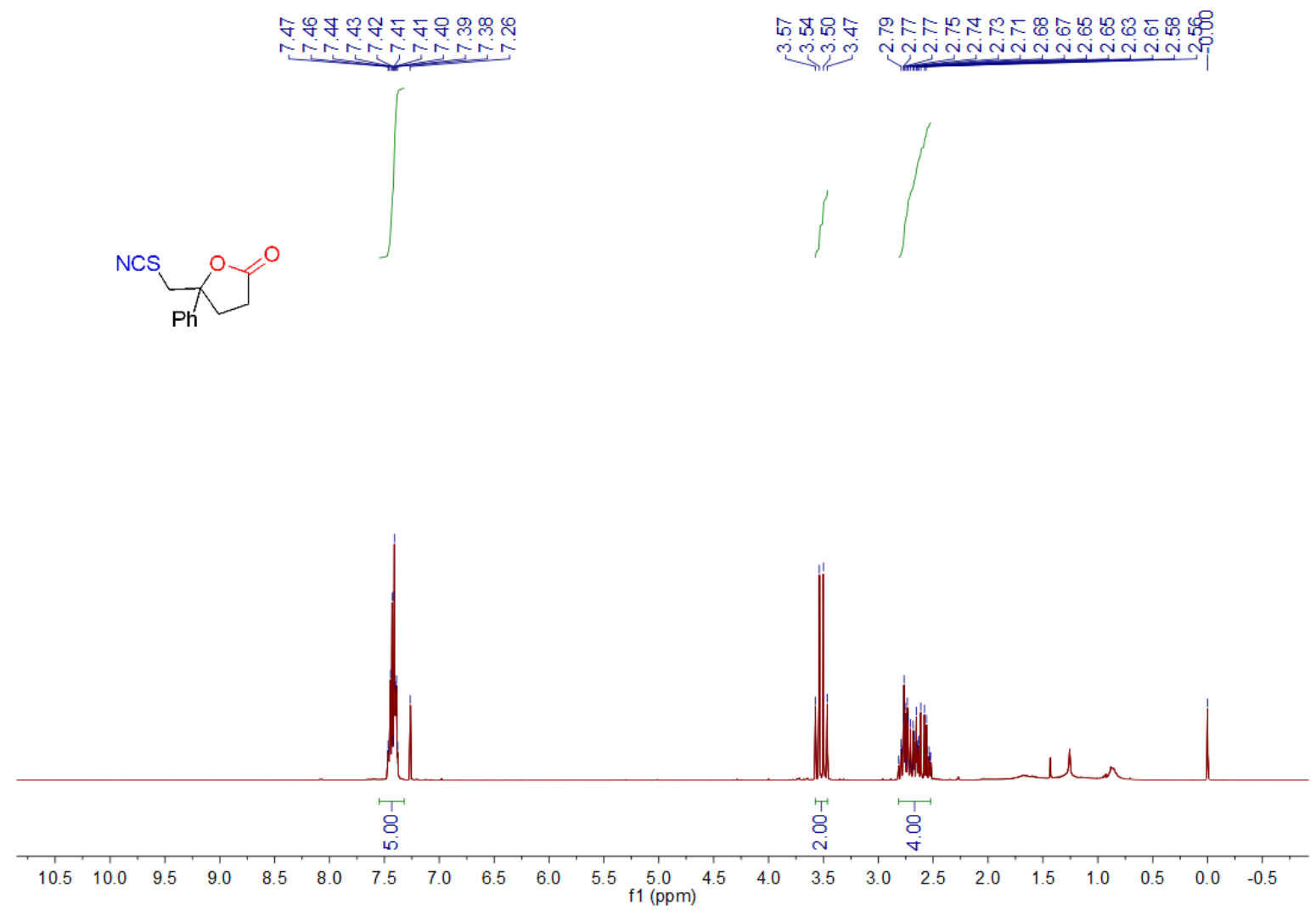

${ }^{13} \mathrm{C}$ NMR (101 MHz, $\mathrm{CDCl}_{3}$ ) spectrum of 5-Phenyl-5-(thiocyanatomethyl)dihydrofuran-2(3H)-one (2x)

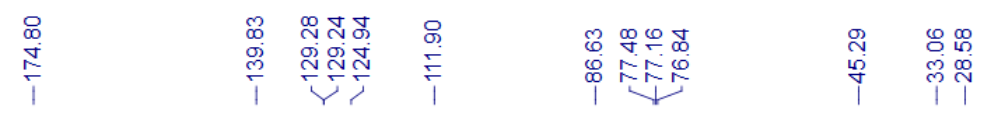

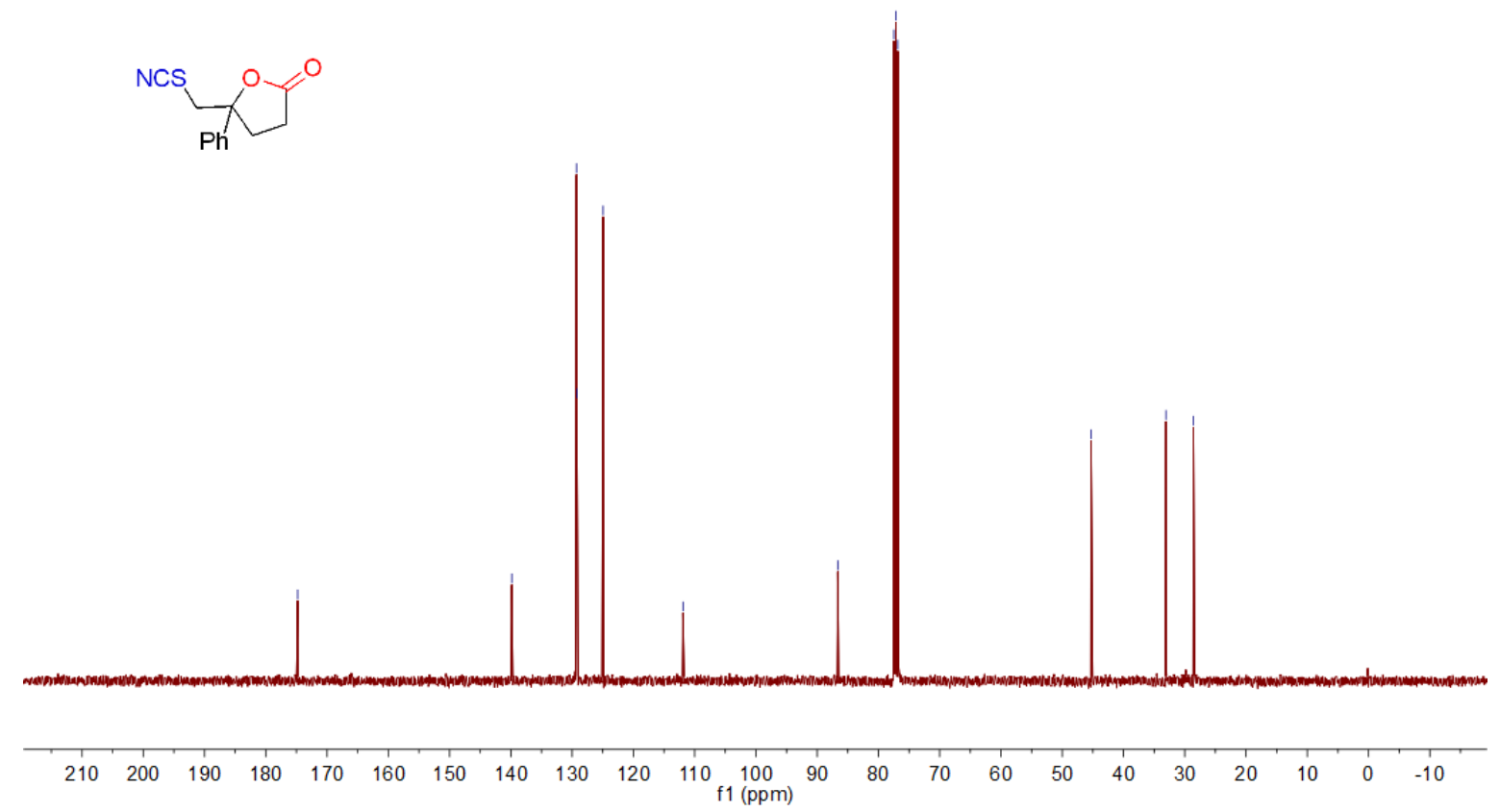


${ }^{1} \mathrm{H}$ NMR (400 $\left.\mathrm{MHz}, \mathrm{CDCl}_{3}\right)$ spectrum of 1-((4-Nitrophenyl)sulfonyl)-2-(1-((trifluoromethyl)thio)pentyl) pyrrolidine (4)

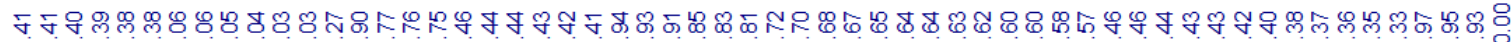
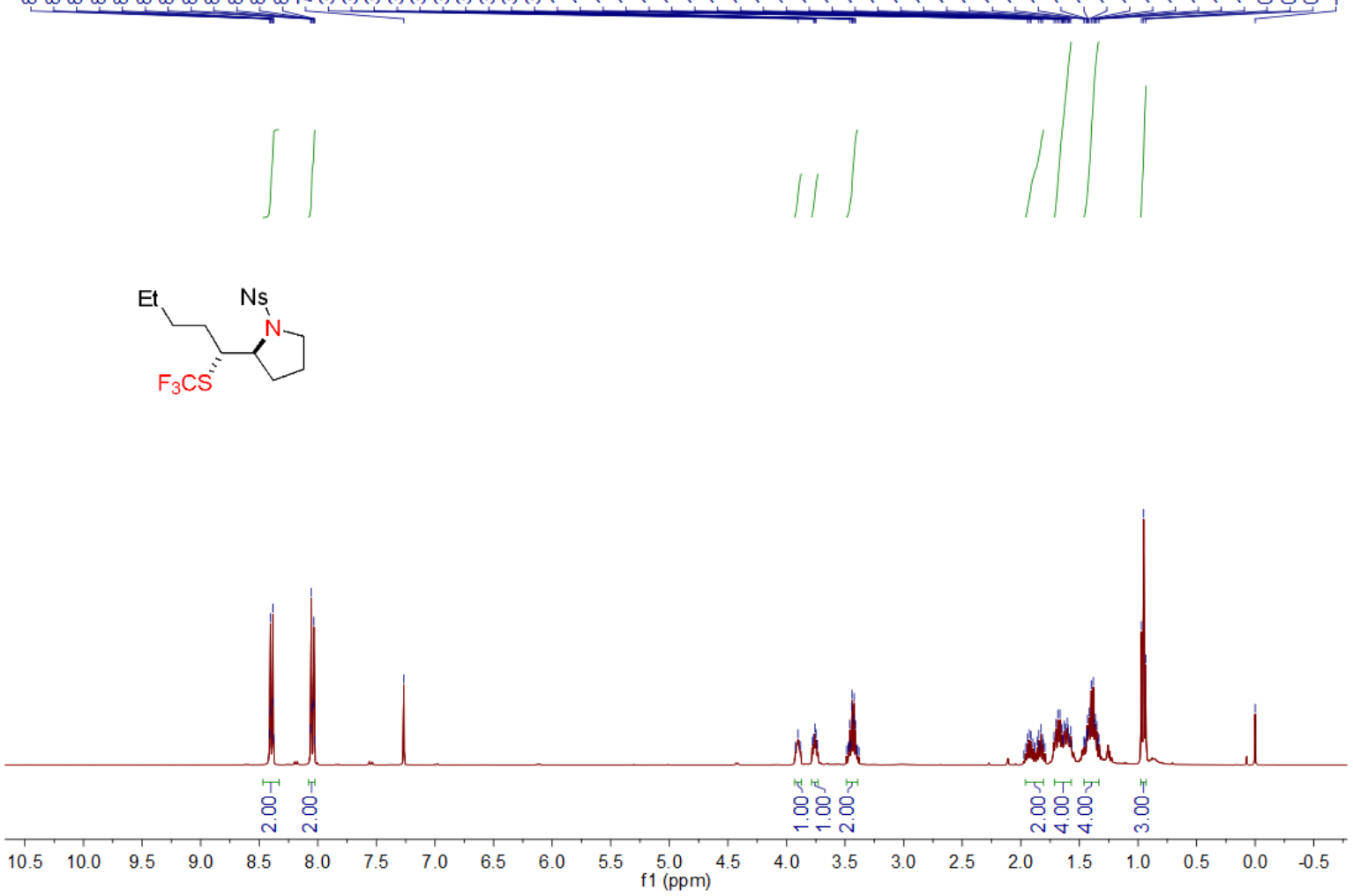

${ }^{13} \mathrm{C}$ NMR (101 $\left.\mathrm{MHz}, \mathrm{CDCl}_{3}\right)$ spectrum of 1-((4-Nitrophenyl)sulfonyl)-2-(1-((trifluoromethyl)thio)pentyl) pyrrolidine (4)

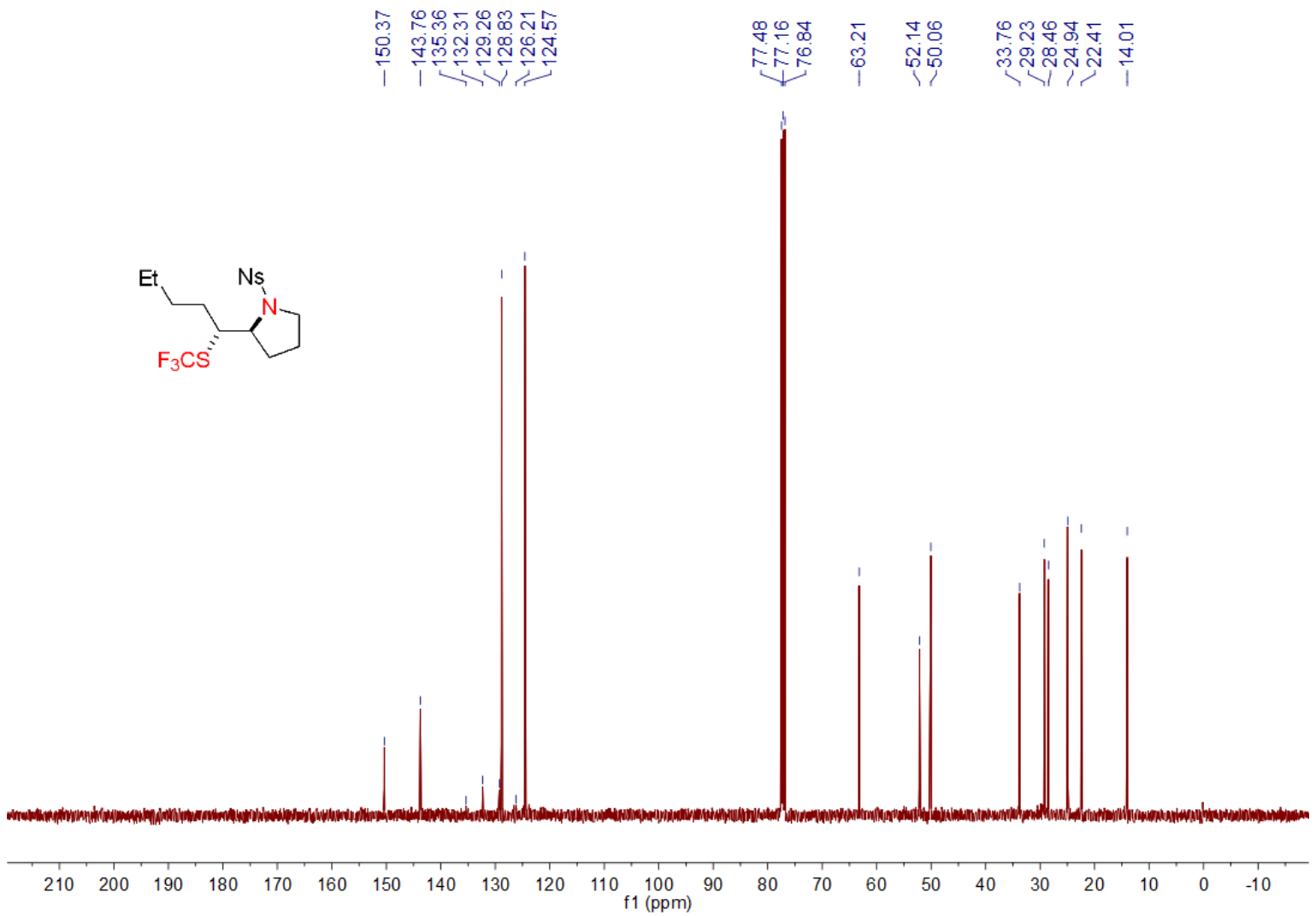


${ }^{1} \mathrm{H}$ NMR (400 $\left.\mathrm{MHz}, \mathrm{CDCl}_{3}\right)$ spectrum of $\boldsymbol{O}$-Methyl-(1-(1-((4-nitrophenyl)sulfonyl)pyrrolidin-2-yl)pentyl) carbonimidothioate (5)
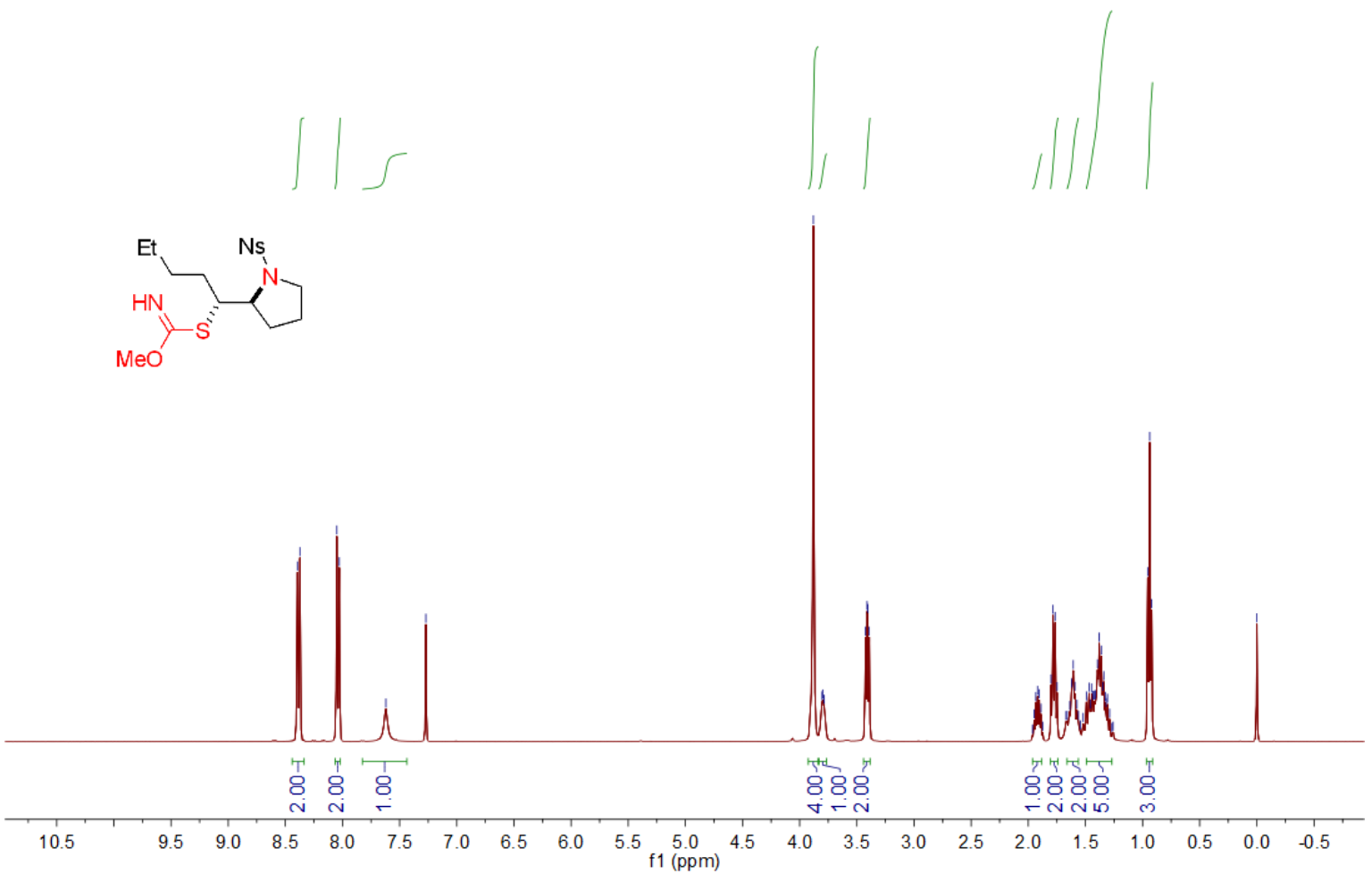

${ }^{13} \mathrm{C}$ NMR (101 $\left.\mathrm{MHz}, \mathrm{CDCl}_{3}\right)$ spectrum of $\boldsymbol{O}$-Methyl-(1-(1-((4-nitrophenyl)sulfonyl)pyrrolidin-2-yl)pentyl) carbonimidothioate (5)

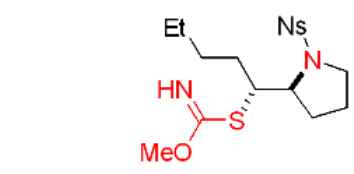

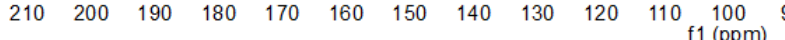




\section{References}

(1) (a) Wu, D.; Qiu, J.; Karmaker, P. G.; Yin, H.; Chen, F.-X. J. Org. Chem. 2018, 83, 1576. (b) Toste, F. D.; Stefano, V. D.; Still, I. W. J. Synth. Commum. 1995, 25, 1277. (c) Qiu, J.; Wu, D.; Karmaker, P. G.; Yin, H.; Chen, F.-X. Org. Lett. 2018, 20, 1600. (d) Ye, A.-H.; Zhang, Y.; Xie, Y.-Y.; Luo, H.-Y.; Dong, J.-W.; Liu, X.-D.; Song, X.-F.; Ding, T.; Chen, Z.-M. Org. Lett. 2019, 21, 5106. (e) Xu, C.; Ma, B.; Shen, Q. Angew. Chem. Int. Ed. 2014, 53, 9316.

(2) (a) An, R.; Liao, L.; Liu, X.; Song, S.; Zhao, X. Org. Chem. Front. 2018, 5, 3557. (b) Du, B.; Jin, B.; Sun, P. Org. Lett. 2014, 16, 3032. (c) Dong, Z.-B.; Balkenhohl, M.; Tan, E.; Knochel, P. Org. Lett. 2018, 20, 7581.

(3) (a) Zhou, L.; Tay, D. W.; Chen, J.; Leung, G. Y. C.; Yeung, Y.-Y. Chem. Commun. 2013, 49, 4412. (b) Mancheno, D. E.; Thornton, A. R.; Stoll, A. H.; Kong, A.; Blakey, S. B. Org. Lett. 2010, 12, 4110. (c) Hazelden, I. R.; Carmona, R. C.; Langer, T.; Pringle, P. G.; Bower, J. F. Angew. Chem. Int. Ed. 2018, 57, 5124. (d) Farndon, J. J.; Young, T. A.; Bower, J. F. J. Am. Chem. Soc. 2018, 140, 17846. (e) Zhu, Z.; Luo, J.; Zhao, X. Org. Lett. 2017, 19, 4940.

(4) (a) Luo, J.; Liu, Y.; Zhao, X. Org. Lett. 2017, 19, 3434. (b) Kaga, H.; Goto, K.; Takahashi, T.; Hino, M.; Tokuhashi, T.; Orito, K. Tetrahedron 1996, 52, 8451.

(5) (a) Cao, Q.; Luo, J.; Zhao, X. Angew. Chem. Int. Ed. 2019, 58, 1315. (b) Brozek, L. A.; Ardolino, M. J.; Morken, J. P. J. Am. Chem. Soc. 2011, 133, 16778.

(6) Guo, R.; Huang, J.; Huang, H.; Zhao, X. Org. Lett. 2016, 18, 504. 